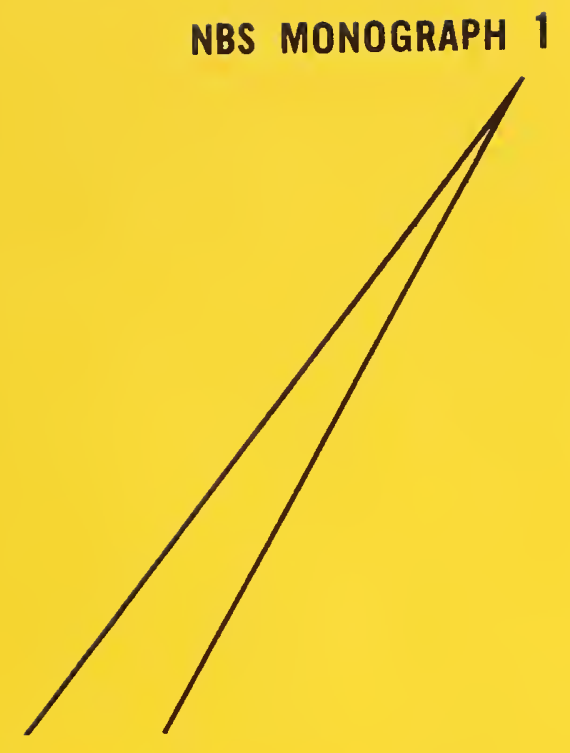

\title{
Energy Dissipation by Fast Electrons
}

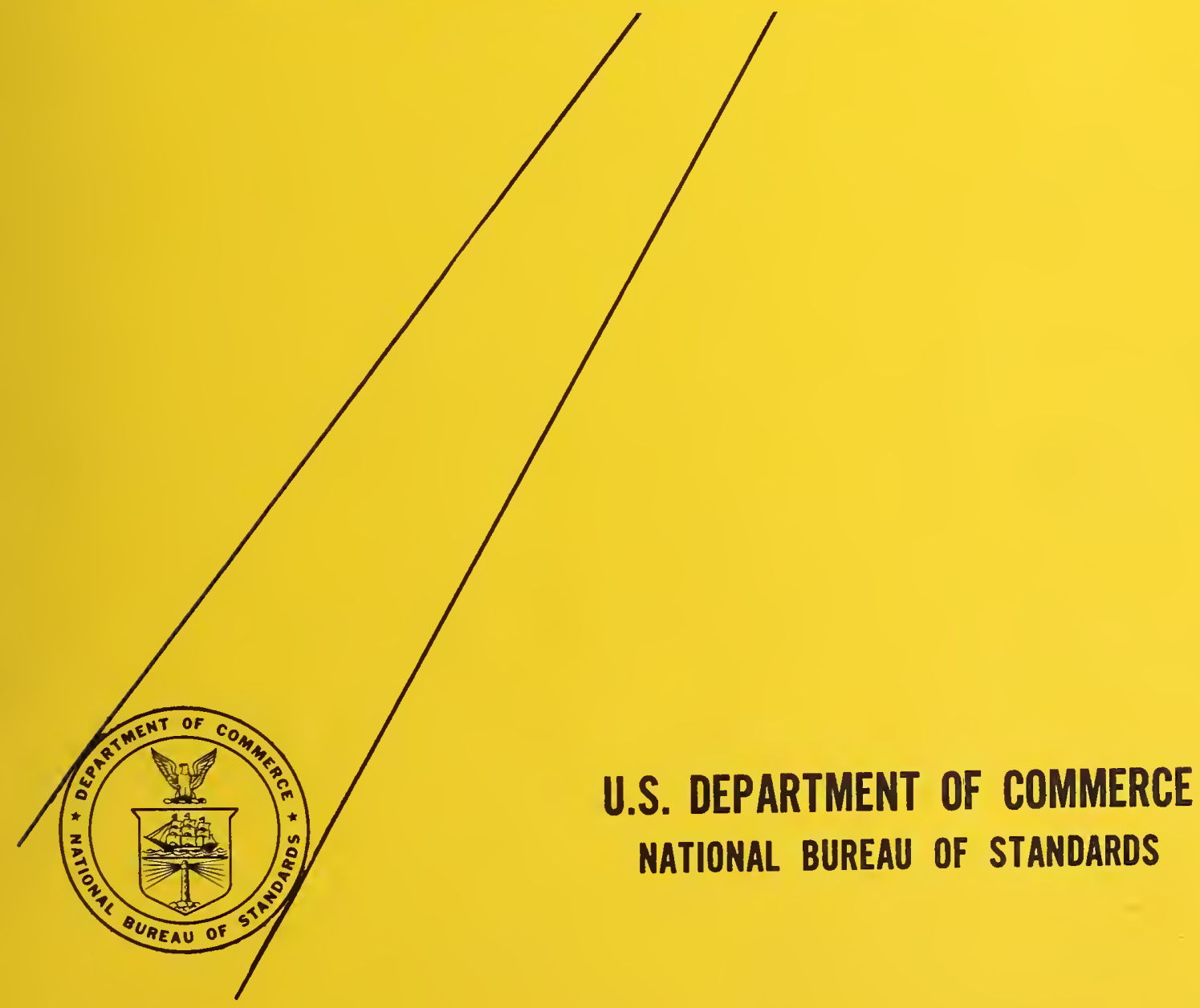



Addendum for

NBS Monograph No. 1

Energy Dissipation by Fast Electrons

Page

2

4

8

Eq. 31

8

4 from bottom

$\{x 1+\pi(z / 137)$

$I_{n 0}^{n+i-\frac{3}{2}}$

Eq. 15
Should read

detail in [1], we only

$I_{n 0}^{n+i-1}$

$\{[1+\pi(z / 137) x$

$(\beta \cos \chi \sqrt{2})]$

$\left.\frac{\Gamma\left(1+i \frac{z}{137 \beta}\right)}{\Gamma\left(1-i \frac{z}{137 \beta}\right)}\right\}$

$0.100000 E-01$,

11

4 from bottom

$0.1000000-01$, 
UNITED STATES DEPARTMENT OF COMMERCE - Frederick H. Mueller, Secrelary NATIONAL BUREAU OF STANDARDS - A. V. Astin, Director

\title{
Enerǵy Dissipation by Fast Electrons
}

\author{
L. V. Spencer
}

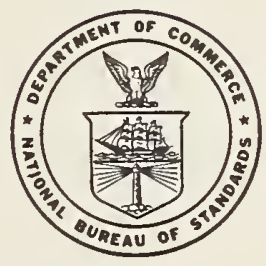

National Bureau of Standards Monograph 1

Issued September 10, 1959

For sale by the Superintendent of Documents, U.S. Government Printing Office Washington 25, D.C. - Price 45 cento 


\section{Contents}

1. Introduction

2. Methods and input information

2.1. Calculation of moments...

2.2. Calculation of the energy dissipation distribution from its moments...- 5

2.3. Choice and preparation of input data.................... 8

3. Tabulations and their description

3.1. Description of the tables............... 10

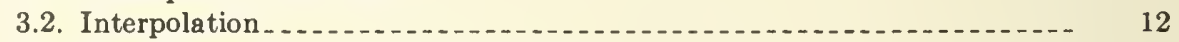

3.3. Comparisons with experimental data

4. References_... 70

II 


\title{
Energy Dissipation by Fast Electrons
}

\author{
L. V. Spencer
}

\begin{abstract}
Tabulations are given of the energy dissipated by fast electrons at different distances from monoenergetic electron sources, for plane perpendicular and point isotropic sources. A summory of the theoretical methods and data utilized, and a table of spatial moments are also included.
\end{abstract}

\section{Introduction}

This Monograph belongs to a series of reports on radiation physics data being prepared with the support of the Office of Naval Research and the Atomic Energy Commission.

Earlier reports of this series were designed in part to present input data necessary for the preparation of this report, which gives results of extensive calculations of electron penetration taking into account both nuclear elastic scattering and electron slowing down. The tabulations given here are designed to answer the following type of problem: If electrons of kinetic energy $E_{0}$ are produced at a point or on a plane in a material with atomic number $Z$, the electrons will travel away from their point of origin, dissipating energy to the material as they go. Eventually each electron will have completely given up its initial energy. What will be the spatial distribution of the energy transferred to the surrounding material?

Two types of electron sources are considered, namely point isotropic sources emitting electrons of energy $E_{0}$ equally in all directions, and plane sources emitting electrons of energy $E_{0}$ only in a single direction which is perpendicular to the source plane. Superposition of the point source data for different $E_{0}$ will give data for more complete source spectra, such as the beta-ray spectra from a radioactive isotope or from mixed fission products. Superposition of the point sources at different spatial locations will give results descriptive of surface and volume beta-ray source geometries. Similarly, the data for the plane perpendicular source may be applied to the case of a beam of electrons injected into a material, since the integral of the energy deposition on any plane perpendicular to the beam is independent of the beam width and can therefore be calculated as though the beam were infinitely wide, thus constituting a plane perpendicular source.

The calculations presented here were programed for the IBM 704 digital computer at NBS. The methods which were used were essentially those outlined in [1].' Results are given for $E_{0}$ varying in approximately logarithmic intervals from 0.025 to $10 \mathrm{Mev}$, for the following materials: $\mathrm{C}, \mathrm{Al}, \mathrm{Cu}, \mathrm{Sn}$, $\mathrm{Pb}$, air, and polystyrene. In the case of $\mathrm{Sn}$ and $\mathrm{Pb}$, some of the lower and some of the higher values of $E_{0}$ have been omitted, the lower values because the range and cross-section data are unreliable near the atomic binding energies, the higher values because radiation straggling, which has not been taken into account, tends to dominate at high energies.

The medium is assumed to be homogeneous, extending in all directions around the source a distance greater than the electron range. The electrons are assumed to slow down continuously until their initial kinetic energy has been completely exhausted. The neglect of range straggling due to large singleenergy losses occurring in both radiative and inelastic collisions makes the data somewhat inaccurate; but the errors are probably important only for very large distances from the source, where only a minor fraction of the energy is dissipated. Since a major effort is required to improve the results further, and since experimental studies show generally good agreement where comparisons have been made, it is felt that the data are of sufficient accuracy and usefulness to justify their publication.

1 Figures in brackets indicate the literature references at the end of this Monograph. 
The remainder of this Monograph is divided into two parts, the first outlining the methods and data which were used to make the calculations and the second presenting and describing the tabular data. Section 3 describing the tabular data, has been made almost completely self-contained so that persons who need data but do not wish to follow the derivations may turn immediately to this latter section and find all the information they require.

\section{Methods and Input Information}

\subsection{Calculation of Moments}

Since the basic theoretical methods used to calculate electron energy dissipation distributions have already been given in considerable detail in [1]. We only sketch the arguments and procedures here.

The assumption that electrons lose their energy continuously established a relation between the residual range $r$ of the electrons, measured along their path, and the average rate of energy loss, i.e., the stopping power $(d E / d r)$ :

$$
r=\int_{0}^{E} \frac{d E}{d E / d r}
$$

where $0<E<E_{0}$ is the kinetic energy of an electron which has slowed down but not lost its energy completely. This equation is the basis for the tabulations of electron ranges [2].

It is convenient to measure both distances and residual ranges in units of $r_{0}=r\left(E_{0}\right)$. For an electron source located on the plane $z=0$ which emits electrons of kinetic energy $E_{0}$ in the direction perpendicular to this plane and towards increasing $z$, the distance of a point from the source plane will be conveniently identified by a dimensionless parameter $x=z / r_{0}$. Similarly, we replace the residual range $r$ by a dimensionless ratio $t=r / r_{0}$. We define the flux of electrons, $I(t, \theta, x) 2 \pi d(\cos \theta) d t$, as the number of electrons per second that cross a small spherical probe of unit cross sectional area at a distance $x$ from the source, having residual ranges between $t$ and $t+d t$ and obliquities between $\theta$ and $\theta+d \theta$ relative to the direction from the source to the probe.

The electron flux must satisfy a transport equation, which has the following form in steady state conditions for the plane perpendicular source:

$$
-\frac{\partial I}{\partial t}+\cos \theta \frac{\partial I}{\partial x}=\int_{0}^{2 \pi} d \varphi^{\prime} \int_{-1}^{1} d\left(\cos \theta^{\prime}\right) S(t, \theta)\left\{I\left(t, \theta^{\prime}, x\right)-I(t, \theta, x)\right\}+\frac{1}{2 \pi} \delta(x) \delta(t-1) \delta(\cos \theta-1),
$$

where $\theta$ is the angle between the electron directions $\left(\theta^{\prime}, \phi^{\prime}\right)$ and $(\theta, \phi)$ before or after a collision. This is basically a continuity equation. The terms on the left describe a rate of change in $I(t, \theta, x)$ due to slowing down (first term) and due to spatial displacement (second term). The terms on the right describe a rate of change in the flux due to elastic collisions (first term) and due to generation of electrons by the source. The last term, describing a unit-electron source current, is the product of three Dirac delta functions which guarantee that the electrons are generated only at $x=0, t=1$, and $\cos \theta=1$. If the source is constant with time, $I(t, \theta, x)$ must also be constant with time, and therefore the rates of change in this quantity must balance one another.

The function $S(t, \theta)$ which appears in the first term on the right of (2) requires a precise definition: The quantity $2 \pi S(t, \theta) d(\cos \theta) d t$ is the probability that an electron having kinetic energy corresponding to $t$ will undergo, while slowing down from $t+d t$ to $t$, an elastic collision which results in a deflection through an angle between $\theta$ and $\theta+d \theta . S(t, \theta)$ is equal to $r_{0} N \sigma(E, \theta)$, where $N$ is the number of atoms per gram of material, $E$ is the kinetic energy corresponding to $t, \sigma$ is the elastic scattering cross section in a centimeters squared per steradian, per atom, and $r_{0}$ is measured in grams per square centimeter.

The procedure for solving (2) utilizes an expansion in spatial moments and spherical harmonics. Thus, if

$$
I_{n l}(t)=\int_{-1}^{1} d x x^{n} \int_{-1}^{1} 2 \pi d(\cos \theta) P_{l}(\cos \theta) I(x, \theta, t)
$$

and

$$
S_{l}(t)=\int_{-1}^{1} 2 \pi d(\cos \theta)\left[1-P_{l}(\cos \theta)\right] S(t, \theta),
$$


an interlinked system of equations for the $I_{n l}(t)$ can be derived from (2):

$$
-\frac{\partial I_{n l}(t)}{\partial t}+S_{l}(t) I_{n l}(t)=\frac{n}{2 l+1}\left\{(l+1) I_{n-1, l+1}(t)+l I_{n-1, l-1}(t)\right\}+\delta_{n 0} \delta(1-t) .
$$

Further analysis is dependent upon the fact that the scattering coefficients $S_{l}(t)$ can be obtained accurately from the suitable formula

$$
S_{l}(t)=\frac{\alpha d_{l}}{t(t+\alpha)}
$$

where $d_{l}$ and $\alpha$ are constants. This analytic form, which is accurate over a very wide range of $t, Z$, and $E_{0}$ values, is made plausible by a derivation due to $\mathrm{C}$. Blanchard which is given in appendix $A$ of [1]. Expression (6) makes it possible to obtain from (5) a set of recursion relations linking "residual range moments" $I_{n l}^{m}$ defined by

$$
I_{n l}^{m}=\int_{0}^{1} d t\left[\frac{(1+\alpha) t}{t+\alpha}\right]^{m-n-1 / 2} I_{n l}(t) .
$$

Derivation of the recursion relations satisfied by the $I_{n l}^{m}$ will not be given, but it is essentially that outlined in [1, Appendix C] with $m=p+n+1 / 2$, and $I_{n l}^{m}=(1+\alpha)^{p} \Phi_{n l}^{p}$. They are

$$
\begin{aligned}
I_{n l}^{m}=n \frac{\alpha}{1+\alpha} \sum_{i=0}^{\infty} \frac{(i+1)}{\left[d_{l}-n+1 / 2+m+i\right]} \frac{1}{(1+\alpha)^{i}}\left\{\frac{l+1}{2 l+1} I_{n-1, l+1}^{m+i}+\frac{l}{2 l+1} I_{n-1, l-1}^{m+i}\right\} & +\delta_{n 0} \frac{\alpha}{1+\alpha} \sum_{i=0}^{\infty} \frac{(i+1)}{\left[d_{l}+1 / 2+m+i\right]} \frac{1}{(1+\alpha)^{i}} .
\end{aligned}
$$

The system of eq (8) has a complicated appearance, but it contains one fundamental simplifying feature, namely that all the terms appearing in each equation have superscripts $m^{\prime} \geq m$. This means that a knowledge of the $I_{n l}^{m^{\prime}}$ for $m^{\prime}>m$ makes possible a solution for $m$ and then chainwise for lower values of $m$. The procedure for solving eq (8) is based on this feature, together with the fact that all the sums converge essentially like a geometric series. By starting the solution at sufficiently large $m_{\text {initial }}=M$, it becomes unimportant whether or not terms $m^{\prime} \geq M$ are accurately represented, since they contribute a negligible amount to the low values of $m$ which are of primary interest.

The first step in the machine programing consists of a numerical determination of the $I_{n l}^{m}$ through solution of (8). For this purpose it is convenient to restate (8) in a simpler form. We make the following definitions:

$$
\begin{aligned}
T_{n l}^{m}=n\left(\frac{\alpha}{1+\alpha}\right) \frac{1}{\left[d_{l}-n+1 / 2+m\right]} & \left\{\frac{l+1}{2 l+1} I_{n-1, l+1}^{m}+\frac{l}{2 l+1} I_{n-1, l-1}^{m}\right\}, \text { for } n>0 \\
T_{0 l}^{m}= & \left(\frac{\alpha}{1+\alpha}\right) \frac{1}{\left[d_{l}+1 / 2+m\right]}, \\
C_{n l}^{m} & =\sum_{i=0}^{\infty}\left(\frac{1}{1+\alpha}\right)^{i} T_{n l}^{m+i} .
\end{aligned}
$$

In terms of these quantities, and by means of the summation device [1, eq 27], we can rewrite (8) in the form of coupled equations to be evaluated successively in the order stated below:

$$
\begin{aligned}
& C_{n l}^{m}=T_{n l}^{m}+\frac{1}{1+\alpha} C_{n l}^{m+1}, \\
& I_{n l}^{m}=C_{n l}^{m}+\frac{1}{1+\alpha} I_{n l}^{m+1} .
\end{aligned}
$$

Equations (9) and (10) exhibit more clearly the progression of the solution from large to small values of $m$. It is clear that given initial values $T_{n l}^{M}, C_{n l}^{M}$, and $I_{n l}^{M}$, these same quantities may be deter- 
mined in turn for $M-1, M-2$, etc., down to $m=0$. To complete the analysis, therefore, it is necessary to give some device for estimating the initial values. 'Two choices have been used: In some cases, particularly for large $\alpha$, the $I_{n l}^{M}$ and $C_{n l}^{M}$ were set equal to zero. More frequently, a crude estimate of these quantities was used, which was based on approximate evaluation of the sums in (8) for large $M$, namely

$$
\begin{gathered}
C_{n l}^{M}=\left(\frac{1+\alpha}{\alpha}\right) T_{n l}^{M}, \\
I_{n l}^{M}=\left(\frac{1+\alpha}{\alpha}\right)^{2} T_{n l}^{M} .
\end{gathered}
$$

The expressions (11) break the $M$ linkages and permit calculation of approximate values for $I_{n l}^{M}$ and $C_{n l}^{M}$ with as many $n, l$ combinations as desired.

The foregoing equations are now almost ideally suited for machine computation. Because of the rapidity of the computer operations, there is hardly any limitation to the values of $M$ which are practical. After some preliminary exploration, $M$ was made sufficiently large that any further increase would not change the results for small $m$ significantly in the eighth place. For most of the calculations $M=50$ proved sufficient for this, but for energies $E_{0} \geq 2 \mathrm{Mev}, M=150$ was used. As a final check, comparisons were made with values of $I_{00}^{0}$ obtained from $(7)$, with $I_{00}(t)=1$,

$$
\begin{aligned}
I_{00}^{0} & =1+\frac{1}{2} \frac{\alpha}{\sqrt{\alpha+1}} \ln \left(\frac{\sqrt{\alpha+1}+1}{\sqrt{\alpha+1}-1}\right), & & \alpha>0, \\
& =1+2 \frac{|\alpha|}{\sqrt{|\alpha|-1}}\left\{\frac{\pi}{4}-\frac{1}{2} \tan ^{-1} \sqrt{|\alpha|-1}\right\}, & & \alpha<-1 .
\end{aligned}
$$

Calculation of numerical values for $I_{n 0}^{M}$ represents a major objective of the machine program, since these numbers describe the total flux of electrons integrated over all directions. The procedure just described for obtaining them has proved very rapid and satisfactory.

Once the $I_{n 0}^{m}$ have been obtained, we are in a position to calculate the energy dissil: tion distribution $I(z)$, which is the end product of our investigation. If $I(z) d z$ is the energy dissipated per square centimeter in the plane layer between $z$ and $z+d z$, then $I(z)$ is an integral over $I_{0}(t, x)$, namely

$$
I(z)=\frac{1}{r_{0}} \int_{0}^{r_{0}} d r\left(\frac{d E}{d r}\right) I_{0}\left(r / r_{0}, z / r_{0}\right)
$$

For our purposes it is advantageous to rewrite (13) in scaled form in terms of a de-dimensionalized function $J(x)=I(z) /(d E / d r)_{E_{0}}$ :

$$
J(x)=\int_{0}^{1} d t \epsilon(t) I_{0}(t, x)
$$

where $\epsilon(t)=(d E / d r) /(d E / d r)_{E_{0}}$. Note that $\epsilon(t)=1$ at $t=1$. Equations (13) and (13') express the assumption that each electron contributing to the flux $I_{0}(t, x)$ at $x$ dissipates energy at the average rate $d E / d r$ per unit pathlength traveled.

Our procedure is to calculate first the spatial moments $J_{n}$ of $J(x)$, and from these obtain the function itself. 'To obtain the $J_{n}$ we use an approximate analytical representation for $\epsilon(t)$, namely

$$
\epsilon(t) \approx \sum_{i=1}^{4} A_{i}\left[\frac{(1+\alpha) t}{t+\alpha}\right]^{i-\frac{3}{2}} .
$$

Inserting this expression into $\left(13^{\prime}\right)$, and then calculating spatial moments of both sides, we obtain an expression for $J_{n}$ in terms of the $I_{n 0}^{m}$ :

$$
J_{n}=\sum_{i=1}^{4} A_{i} I_{n 0}^{n+t-\frac{3}{2}}
$$


The representation (14) contains four constants $A_{i}$, which require four conditions for their determination. Since $\epsilon(t)$ is a ratio of stopping powers, it can be evaluated numerically for different $t_{j}$ values. We may then require our representation to equal $\epsilon\left(t_{j}\right)$ at $t=t_{j}$. Each value of $j$ then corresponds to one condition to be fulfilled by our representation. By a suitable choice of $t_{j}$ values, the representation can be made to resemble closely the actual function $\epsilon(t)$. For this purpose, the values $t_{1}=1, t_{2} \approx 0.5$, and $t_{3} \approx 0.1$ were selected. Instead of selecting a fourth condition similar to these three, however, the condition was chosen that the total energy dissipated must always equal the initial kinetic energy $E_{0}$ of the electrons. This is expressed by the integral

$$
J_{0}=\int_{-1}^{1} d x \int_{0}^{1} d t \epsilon(t) I_{0}(t, x)
$$

which takes the form

$$
J_{0}=\sum_{i=1}^{4} A_{i} I_{00}^{1-\frac{3}{2}}=\frac{E_{0} / r_{0}}{(d E / d r)_{E_{0}}}
$$

This condition insures that the stopping power is correctly expressed "on the average"; and it partially compensates for inaccuracies in (14) which arise from the difficulty of representing $(d E / d r)$ simultaneously at low and high energies. When combined with the other three expressions obtained from (4) by setting $t=t_{j}$, we have a set of simultaneous linear equations whose solution yields the $A_{i}$. A short machine program was included to accomplish this and then, with the results, to evaluate the sums (15).

In summary, the machine program for calculating moments $J_{n}$ of the energy dissipation distribution $J(x)$ consists of a section which solves the moment eqs (8) by means of the recursion eq (9) to (11), a section to determine the $A_{i}$, and a final section to perform the sums (15). As input data, values of the constants $d_{l}, \alpha$, and $M$ are required for the first section, and $E_{0}, r_{0},(d E / d r)_{E_{0}}, t_{1}, t_{2}$, and the values $(d E / d t)_{t \approx 0.5}$ and $(d E / d t)_{\imath \approx 0.1}$ for the second section.

The preceding discussion outlines the calculation of moments $J_{n}$ for the plane perpendicular source. Also of interest are the moments for the plane isotopic source from which one can obtain the moments of the point isotropic sources (see eq 28). Note that in the case of the plane isotropic source, each element of area on the source plane emits electrons equally in all directions. Only minor modifications are needed in the equations already given to obtain equations from which a calculation of plane isotropic source moments can be made. In particular, the factor $\delta(\cos \theta-1)$ must be replaced by $\frac{1}{2}$ in the last term of (2), a factor $\delta_{20}$ must be included in the last term of (5) and (8), and in (9) the expression for $T_{0 l}^{m}$ must also include a factor $\delta_{l 0}$. Otherwise all equations are identically the same for both source types.

\subsection{Calculation of the Energy Dissipation from its Moments}

The method which was used to calculate the energy dissipation distributions was the "function fitting" method outlined in [1], in which several terms are added together to represent the distribution, all terms having the same functional form but differing in the value of a scale parameter. The representation is expected to be accurate if its moments agree with the known moments of the distribution, if the functional form has been well chosen, if it is smooth, nonoscillatory, positive, single-peaked, and if it is correct at $x=0$.

The chief advantage and also the chief difficulty with this method for calculating a function from its moments arise from its all-or-nothing character. To obtain values for all the scale parameters and "strength" parameters in the representation, it is necessary to solve simultaneously a set of nonlinear equations. The solution of these equations may be acceptable on the basis of the above-mentioned criteria. In this case the representation is expected to be very accurate. On the other hand, solution of the equations may lead to coefficients which are complex, too large, or too small, and which give a representation which is uneven or oscillatory in nature and not representative of the desired distribution at all. Because the solution of the equations may "fail" in this way, perhaps more often than it may succeed, the function-fitting method is not well suited to digital computer operations. Nevertheless, because only this method had proved successful in hand computations, it was necessary to attempt its use in systematic machine computations. 
The expectations that frequent failures would be encountered with any function-fitting machine program led us to take three precautions. (1) The functional form selected contained a number of parameters which could be given vahes arbitrarily. This was to make possible a wide variety of modifications in this function without having to write new codes. It turned out that many of these arbitrary parameters were unnecessary. (2) In each machine calculation, at least five different attempts were made to calculate the distribution, each attempt corresponding to a different value of one of the arbitrary parameters. Thus, several tabulations of the distribution were usually obtained in the same machine rum. (3) Where several comparable tabulations occurred, these were graphed and compared both visually and in regard to their analytic form. A choice was made of the distribution best satisfying the criteria. It ahmost always turned out that if two or more such approximate distributions agreed about equally well with the basic criteria, they agreed with each other to within 1 or 2 percent, except for very small $J(x)$.

Calculation of the spatial distributions proceeded as in [1]. Even and odd moment sets were considered as descriptive of even and odd functions $J^{\text {even }}(x)$ and $J^{\text {odd }}(x)$, which yield $J(x)$ through the combinations

$$
\begin{aligned}
J(x) & =1 / 2\left[J^{\text {even }}(x)+J^{\text {odd }}(x)\right], \\
J(-x) & =1 / 2\left[J^{\text {even }}(x)-J^{\text {odd }}(x)\right] .
\end{aligned}
$$

The functions $J^{\text {even }}(x)$ and $J^{\text {odd }}(x)$ were calculated from the following analytic expressions:

$$
\begin{aligned}
& \left(1-a_{0} x^{2}\right) J^{\text {even }}(x)=\sum_{i=0}^{3} \alpha_{i} \beta_{i}\left(a_{1}-a_{2} \frac{x}{\beta_{i}}\right)\left(1-\frac{x}{\beta_{i}}\right)^{\gamma} e^{-A x /\left(\beta_{i}-x\right)}, \\
& \left(1-a_{0} x^{2}\right) J^{\text {odd }}(x)=\sum_{i=0}^{3} \alpha_{i}\left(a_{1}-a_{2} \frac{x}{\beta_{i}}\right)\left(1-\frac{x}{\beta_{i}}\right)^{\gamma} e^{-A x /\left(\beta_{i}-x\right)},
\end{aligned}
$$

where the constants $a_{i}$ were usually assigned the values $a_{0}=1, a_{1}=1$, and $a_{2}=0$. The constant $A$, which controls the asymptotic trend of all the terms, was determined by iteration from the expression

$$
\left.\sqrt{A}=\frac{\ln \left(J_{N-2} / J_{N}\right)}{2\{\sqrt{N+1 / 4+(A / 12)}-\sqrt{N-7 / 4+(A / 12)}}\right\}, N=n_{\text {maximum }} .
$$

The precise value of $A$ is not critical except for large $|x|$, where $J(x)$ is very small; and (21) gives a fairly reasonable value for this constant since it corresponds to a simple but realistic approximate form [1, Section 7 and Appendix E],

$$
J(x) \sim(1-x)^{-3 / 2} e^{-A x /(1-x)} .
$$

The constant $\gamma$ was assigned integral or half-integral values, usually not greater than 1 nor less than -2 . The constant $\beta_{0}$ was given the value unity, and the constant $\beta_{1}$ was given five different values in succession, namely $\sqrt{0.9}, \sqrt{0.6}, \sqrt{0.4}, \sqrt{0.1}$, and $\sqrt{0.04}$, each yielding a separate representation of $J(x)$.

The other six constants in each of the expressions (19) and (20), namely $\alpha_{i}, i=1$ to 4 , and $\beta_{i}, i=3,4$. were determined so that five moment equations obtained from (19) and five from (20) were satisfied. and so that two other features of $J(x)$ were given correctly,

and

$$
J^{\text {odd }}(0)=1 \text {, }
$$

$$
\left(\frac{d}{d x} J^{\text {even }}\right)_{x=0}=-\left.\frac{d}{d t}(\epsilon)\right|_{t=1}
$$

Equation (23), together with the moment equations derived from (20) give the following system of simultaneous equations:

$$
\begin{gathered}
\frac{J_{n}-a_{0} J_{n+2}}{a_{1} \omega_{n}-a_{2} \omega_{n+1}}=\sum_{i=0}^{3} \alpha_{i} \beta_{i}{ }^{n+1}, \quad n=1,3,5,7,9, \\
1=\sum_{i=0}^{3} \alpha_{i},
\end{gathered}
$$


where $\omega_{n}$ indicates a number discussed below. Similarly, (24) together with the moment equations derived from (19) give the system

$$
\begin{gathered}
\frac{J_{n}-a_{0} J_{n+2}}{a_{1} \omega_{n}-a_{2} \omega_{n+1}}=\sum_{i=1}^{3} \alpha_{i} \beta_{i}^{n+2}, \quad n=0,2,4,6,8, \\
\frac{\alpha}{\alpha+1} \sum_{i=1}^{4}(i-3 / 2) A_{2}=\left[a_{2}+a_{1}(\gamma+A)\right] \sum_{i=0}^{3} \alpha_{i} .
\end{gathered}
$$

Note that the $\alpha_{i}$ and $\beta_{i}$ for $J^{\text {even }}$, obtained by solution of (25), are quite different from the corresponding values for $J^{\text {odd }}$, obtained from solution of (26).

The number $\omega_{n}$ appearing in both (25) and (26) is defined by the expression

$$
\omega_{n}=\int_{0}^{1} d x x^{n}(1-x)^{\gamma} e^{-A x /(1-x)}
$$

Machine calculation of these numbers presented an interesting problem, since quite a few are needed, and they must be determined with great accuracy. For this purpose, recursion relations similar to those of $\left[1\right.$, Appendix E] were derived. It was found that by estimating $\omega_{n}$ for a very large $n$, say $n=50$, and then by working back to small values of $n$ using the recursion relations, very precise values could be obtained. Actually, there was more to the problem than this, since accurate absolute values were desired, whereas the method just sketched gives only accurate relative values. In any case, a simple and rapid machine program was devised for this purpose.

Solutions of (25) and (26) were programed for the computer, and the resulting parameters were then used in an additional routine which tabulated $J^{\text {even }}$ and $J^{\text {odd }}$ from (19) and (20). Finally, these tabulations were combined as in (18) to obtain $J(x)$. In the solution of (25) and (26), if $\beta_{2}$ and $\beta_{3}$ turned out to be complex, the remainder of the calculation was omitted and another calculation was attempted with a new value of $\beta_{1}$. Solutions involving complex $\beta_{1}$ were not acceptable because of being inherently oscillatory. Of the many calculations, perhaps half were not completed because of this type of failure.

The preceding discussion is largely descriptive also of the calculations of $J(x)$ for a point isotropic source; but there were a number of differences worth noting. Point isotropic source moments could be easily obtained from plane isotropic source moments, which were actually computed directly, by use of the prescription

$$
J_{n}^{\text {Polnt lootrople }}=(n+1) J_{n}^{\text {Plane lootrople. }}
$$

From these moments $A$ was then determined, using (21). The representation of $J(x)$ for the point isotropic source differed somewhat from (19) because of the added condition that $J(0)=1$. This was ensured by addition of an extra term:

$$
\left(1-a_{0} x^{2}\right) J(x)=\left(1+a_{1} x\right)(1-x)^{\gamma} e^{-A x /(1-x)}+a_{1} \sum_{i=0}^{3} \alpha_{i} \beta_{i}\left(\frac{x}{\beta_{i}}\right)\left(1-\frac{x}{\beta_{1}}\right)^{\gamma} e^{-A x /\left(\beta_{i}-x\right)}
$$

Moments of (29) have the form

$$
\frac{J_{n}-a_{0} J_{n+2}-\left(\omega_{n}+a_{1} \omega_{n+1}\right)}{a_{1} \omega_{n+1}}=\sum_{i=0}^{3} \alpha_{i} \beta_{i}{ }^{n+2}, \quad n=0,2,4,6,8,
$$

where the $\omega_{n}$ are as defined by (27). To complete the set of equations, one more was added to express the condition that

$$
\left.\frac{d}{d x} J(x)\right|_{x=0}=-\left.\frac{d}{d t}(\epsilon)\right|_{i=1}
$$

The parameters $A, \gamma, \beta_{0}$, and $\beta_{1}$ were specified as already indicated, and $a_{0}, a_{1}$ were set equal to unity. It happened repeatedly that none of the solutions obtained from the prearranged set of values of $\beta_{1}$ were acceptable. When this occurred, the parameter next to be given new values was $\gamma$. On occasion it proved necessary to use several different values for $\gamma$ before obtaining an acceptable result; but in no case was it necessary to modify the other parameters. 


\subsection{Choice and Preparation of Input Data}

Three types of input data are required for the calculations outlined in the preceding section, namely electron stopping powers, electron ranges, and elastic scattering cross sections; but none of this information enters directly into the equations which are solved. Instead, a set of derived parameters is used which includes $\alpha$, the $d_{l}$, and the $A_{i}$. 'Thus, after obtaining the basic physical data, a very substantial effort is required to determine the numerical values of the actual equation parameters. In this subsection the physical data is first discussed and then the preparation of the equation parameters is outlined.

Extensive tabulations of range and stopping-power data have been prepared by Nelms [2,3]. We used the data of [2], corrected for density effect according to Sternheimer [4]. This data is based on values for the mean excitation potential obtained by Mather and Segré [5].

The nuclear elastic scattering cross section which was used was essentially the Mott cross section $(\sigma)$ modified to take into account screening by the atomic electrons. Values for the ratio $\sigma / \sigma_{R}$ of the Mott cross section to the Rutherford cross section were taken from the tabulations of Doggett and Spencer [6]. Also used were unpublished values determined by Doggett and Spencer for several additional atomic numbers $Z_{i}$. The screening modification was accomplished through use of Moliére's expression for the screening parameter $\eta$ [7]. To take into account deflections due to collisions with atomic electrons, the nuclear elastic scattering cross section was modified by a factor $\left[\left(Z^{2}+Z\right) / Z^{2}\right](1+\epsilon)$, in accordance with a correction due to Fano [8] to the usual prescription which replaces the factor $Z^{2}$ in the cross section by $Z(Z+1)$.

Due to the remarkable scaling of electron penetration phenomena with the range $r_{0}$ (see, e.g., [1], slowing-down and scattering properties of the medium all find expression through the single function $S(t, \theta)$. For reasons of computational convenience, we represented this function in the form

$$
\begin{gathered}
S(t, \theta)=(3 / 4) N_{A}(Z / A) \phi_{0}(Z+1)(1+\epsilon)\left(E+m c^{2}\right)^{2}\left(m c^{2}\right)^{2}\left[E\left(E+2 m c^{2}\right)\right]^{-2} r_{0}[1+2 \eta-\cos \theta]^{-2}\{\times 1+\pi(Z / 137) \\
\left.(\beta \cos \chi / \sqrt{2})[1+2 \eta-\cos \theta]^{1 / 2}+\left[\left(\sigma / \sigma_{R}\right)-1-\pi(Z / 137) \beta \cos \chi \sin \frac{1}{2} \theta\right][1+2 \eta-\cos \theta]^{2}[1-\cos \theta]^{-2}\right\},
\end{gathered}
$$

where the quantity in curly brackets can be recognized as $\sigma / \sigma_{R}$ to within a minor modification, and

$$
N_{A}=\text { Avogadro's number, }
$$

$\phi_{0}=\frac{8}{3} \pi\left(\frac{e^{2}}{m c^{2}}\right)^{2}=6.65205 \times 10^{-25} \mathrm{~cm}^{2}$ is the Thompson cross section,

$\beta=\left[E\left(E+2 m c^{2}\right)\right]^{\frac{1}{2}} /\left(E+m c^{2}\right)$ is the electron velocity divided by $c$,

$m c^{2}$ is the rest energy of the electron,

$\sigma$ is the Mott scattering cross section,

$$
\begin{aligned}
\epsilon & =(Z+1)^{-1}(\ln 4 \eta)^{-1}\left\{u_{\ln }-\ln \left[0.16 Z^{-2 / 3}\left(1+3.33 \frac{Z}{137 \beta}\right)\right]\right\}, \\
\eta & =\frac{1}{4}\left[\frac{Z^{1 / 3}}{0.885(137)}\right]^{2} \frac{\left(m c^{2}\right)^{2}}{E\left(E+2 m c^{2}\right)}\left[1.13+3.76\left(\frac{Z}{137 \beta}\right)^{2}\right], \\
\cos \chi & =\operatorname{Re}\left\{\frac{\Gamma\left(\frac{1}{2}-i \frac{Z}{137 \beta}\right) \Gamma\left(1+i \frac{Z}{137 \beta}\right.}{\Gamma\left(\frac{1}{2}+i \frac{Z}{137 \beta}\right) \Gamma\left(1-i \frac{Z}{137 \beta}\right.}\right\}, \text { and }
\end{aligned}
$$

$\sigma_{R}=\frac{3}{4} N_{\Lambda}(Z / A) \phi_{0} Z\left(E+m c^{2}\right)^{2}\left(m c^{2}\right)^{2}\left[E\left(E+2 m c^{2}\right)\right]^{-2}(1-\cos \theta)^{-2}$.

In (31), note that $(3 / 4) N_{A}(Z / A) \phi_{0}=0.300 \mathrm{~cm}^{2} / \mathrm{g}$, a tabulation of $\cos \chi$ has been given in [6], and the constant $-u_{\mathrm{In}}$ is a number in the neighborhood of 5 . Everything in (31) is known except the value of 
$-u_{\text {In }}$, which affects only a correction to a correction. To be systematic in "guessing" the value of $-u_{\text {in }}$, the few values in [8] were plotted against $\log Z$ and interpolations were made from a rough curve drawn through the points.

To discuss the further processing of the data, it is convenient to give the different terms in (31) shorter names. In particular, we define $G, C(\Theta), D(\theta)$, and $F(\theta)$ as follows:

$$
\begin{aligned}
& G(E)=(3 / 4) N_{A}(Z / A) \phi_{0}(Z+1)(1+\epsilon)\left(E+m c^{2}\right)^{2}\left(m c^{2}\right)^{2}\left[E /\left(E+2 m c^{2}\right)\right]^{-2} r(E), \\
& C(\Theta)=[1+2 \eta-\cos \theta]^{-2}, \\
& D(\theta)=[1+2 \eta-\cos \theta]^{-3 / 2}, \\
& F(\Theta)=\left\{\left(\sigma / \sigma_{R}\right)-1-\pi(Z / 137 \beta) \cos \chi \sin \frac{1}{2} \theta\right\}(1-\cos \theta)^{-2} .
\end{aligned}
$$

Using these definitions, $S(t, \theta)$ can be written

$$
t S(t, \theta)=G\left\{C(\theta)+\sqrt{\frac{1}{2}} \pi(Z \beta / 137) \cos \chi D(\theta)+F(\theta)\right\} .
$$

The expansion in Legendre coefficients of $C(\theta)$ and $D(\theta)$ is a simple matter and has been given in [1]. On the other hand, $F(\theta)$ is only known numerically, and its harmonic expansion is therefore not so easy to obtain. Our procedure consisted of approximating $F(\theta)$ by a four term polynomial in $(1-\cos \theta)$ over each of the angular ranges $45^{\circ}$ to $90^{\circ}, 90^{\circ}$ to $135^{\circ}$, and $135^{\circ}$ to $180^{\circ}$,

$$
F(\theta)=\sum_{i=0}^{3} f_{i}(1-\cos \theta)^{i-1}
$$

and by a three-term polynomial of the same type over the range $0^{\circ}$ to $45^{\circ}$. Note that in each $45^{\circ}$ range the $f_{t}$ in (34) assume aifferent values. These coefficients were determined so that (34) agreed with the tabulated values of $F(\Theta)$ at $15^{\circ}$ intervals. With the analytic approximations (34), it is possible to accomplish the integrations $(4)$ to obtain coefficients $F_{l}$. The procedure actually followed was that of determining a $12 \times 12$ matrix which, multiplied by the twelve tabulated values $F\left(15^{\circ}\right), F\left(30^{\circ}\right)$, etc., would yield in turn the first twelve harmonic coefficients $F_{1}, F_{2}, \ldots F_{12}$. The matrix multiplications were then performed by machine.

Having determined all the $C_{l}, D_{l}$, and $F_{l}$, the coefficients $S_{l}(t)$ were then calculated from the expression

$$
t S_{l}(t)=G(E)\left\{C_{l}+\sqrt{\frac{1}{2}} \pi(Z \beta / 137) \cos \chi D_{l}+F_{l}\right\}_{E}
$$

for all the energies and elements listed in tables 1 and 2, plus one lower energy, namely $0.01 \mathrm{Mev}$.

In the last stage of the preparation of the data, ratios $S_{l}(t) / S_{1}(t)$ were determined from (35). The representation (6) requires these ratios to be independent of $t$. Actually, they are weakly energy dependent, and to this extent (6) is in error. To minimize the effect of this error, the ratios $d_{l} / d_{1}$ were set equal to $S_{l}(1) / S_{1}(1)$, so that the most penetrating part of the range of the electrons is traveled before the approximation (6) incurs appreciable error:

$$
\frac{d_{l}}{d_{1}}=\frac{S_{l}(1)}{S_{1}(1)}
$$

Finally, to obtain $\alpha$ and $d_{1}$ we notice from (35) that $t S_{1}(t)$ depends only on $E$, not on $E_{0}$. Therefore, according to (6), the quantity

$$
\frac{\alpha d_{1}}{t+\alpha}=t S_{1}(t)
$$

also depends only on $E$, and not on $E_{0}$. This makes it very easy to calculate $\alpha$ and $d_{1}$ from the tabular values of (35). One evaluates (37) for two values of $t$, namely $t=1$ and $t=t_{1}$, corresponding to $E_{0}$ and 
the next lowel energy recorded. Simultaneous solution of these two equations yields $\alpha$ and $d_{1}$ :

$$
\begin{aligned}
& \alpha=\frac{S_{1}(1)-t_{1}\left[t_{1} S_{1}\left(t_{1}\right)\right]}{t_{1} S_{1}\left(t_{1}\right)-S_{1}(1)}, \\
& d_{1}=\frac{1+\alpha}{\alpha} S_{1}(1) .
\end{aligned}
$$

Combination of $d_{1}$ with the ratios $d_{l} / d_{1}$ then give all the $d_{l}$ 's.

This completes the preparation of the data, except for compounds and mixtures, in which case it is necessary to determine the $S_{l}$ from an average over $S_{l}$ values for the constituent elements, in proportion to their fraction of the mixture by weight.

\section{Tabulations and Their Description}

\subsection{Description of the Tables}

The following quantities appear in table 1 :

$Z$, the atomic number; results are given for $Z=6$ (carbon), 13 (aluminum), 29 (copper), 50 (tin), and 82 (lead), and in addition for air and polystyrene. Air is assumed to be 0.755 nitrogen, 0.232 oxygen and 0.013 argon by weight, and polystyrene is taken to be $\mathrm{C}_{8} \mathrm{H}_{8}$.

$E_{0}$, the initial source energy of the electrons; results are given for $E_{0}=0.025,0.05,0.1,0.2,0.4,0.7$, $1,2,4$, and $10 \mathrm{Mev}$, except that some of the highest and lowest values have been omitted in the case of tin and lead. ${ }^{2}$

$(d E / d r)_{\mathrm{E}_{0}}$, the stopping power of electrons at the initial (source) energy.

$r_{0}=r\left(E_{0}\right)$, the residual range of electrons at energy $E_{0}$.

$x$, the distance from the source in units of $r_{0}$. This quantity refers to $z / r_{0}$ for plane perpendicular sources and to $\rho / r_{0}$ for point isotropic sources, where $z$ is measured along a perpendicular to the source plane and $\rho$ is measured radially out from the source point.

$J(x)$, the de-dimensionalized energy dissipation distribution, for plane perpendicular and point isotropic sources, see eqs (13) and $\left(13^{\prime}\right)$. For plane perpendicular sources, $(d E / d r)_{E_{0}} J(x)=I(z)$, where $I(z) d z$ is the energy dissipated per $\mathrm{cm}^{2}$ in the plane layer between $z$ and $z+d z$ by electrons initially given kinetic energy $E_{0}$ assuming 1 electron $/ \mathrm{cm}^{2}$ to be generated at the source plane. ${ }^{3}$ For point isotropic sources, $(d E / d r)_{E_{0}} J(x)=I(\rho)$, where $I(\rho) d \rho$ is the average energy per electron dissipated in the spherical shell between $\rho$ and $\rho+d \rho$. In the case of the plane perpendicular sources, $J(x)$ has been given for positions on both sides of the source plane. Occasional negative values of $J(-x)$ represent spurious effects due to imperfections in the representation. They have been included because they give some guidance regarding the expected accuracy for $x=|-x|$. One does not expect the value $J(x)$ to be more accurate than plus or minus the corresponding value at $-x$, where that value is negative. Note that the discontinuity at the source plane is unity, and that in the case of both point isotropic and plane perpendicular sources the integral over $x$ gives $\left(E_{0} / r_{0}\right) /(d E / d r)_{E_{0}}$.

Along with the tabulated energy dissipation distributions and the associated range and stopping power values at the source energy, we have included many other pieces of data in table 1 in order to give a fairly complete record of the computations. These are:

$(\sigma) / \sigma_{R}(\theta)$, the ratio of the Mott to the Rutherford nuclear elastic scattering cross sections which was used as input data [6];

$S_{l}(1)$, given by (35) for $t=r(E) / r\left(E_{0}\right)=1$, i.e., for $E=E_{0}$;

$d_{1}, \alpha$, quantities characterizing the representation (6) of the scattering coefficients $S_{l}(t)$;

$A_{1}, A_{2}, A_{3}, A_{4}$, the coefficients $A_{t}$ in the analytic approximate representation (14) of the stopping power $\epsilon=(d E / d r) /(d E / d r)_{E_{0}}$,

$A^{\text {PTI }}, A^{\text {PLP }}$, the two values of the constant $A$ in eq (19) to (22) for the point isotropic and plane perpendicular source geometries, respectively. Theoretically the same, these values differ because

\footnotetext{
2 A calculation for $0.1 \mathrm{Mev}$ sourcc cnergy in $\mathrm{Pb}$ has been included to facilitate calculations for energies below $0.7 \mathrm{Mev}$. Since the binding energy of tbe $K$ electrons in $\mathrm{Pb}$ is $\sim 0.07 \mathrm{Mev}$, the numbers in this table are not to be taken seriousiy.

${ }^{3}$ For a heam source, $I(z) d z$ represents the average energy per electron dissipated in the plane layer between $z$ and $z+d z$. 
they were obtained from (21) using different moment sets. The extent of the difference, as it affects the distribution in the manner indicated in (22), serves to indicate a region of large $x$ in which the data are unreliable. There is no simple way to identify this region explicitly, since the representations (19, are more accurate than the expression (22) from which the constants $A$ were determined. But when the difference in $A$ values leads to widely different values for (18), one doesn't expect the tabulated values of $J(x)$ to be accurate. Note that the assumption of continuous energy loss makes $J(x)$ inaccurate in the same region anyway.

In table 2 the moments $I_{n 0}^{p+n+\frac{1}{2}}$ have been recorded which were used to obtain the energy dissipation distributions. According to (7) these represent averages of the residual range function $\left[\frac{(1+\alpha) t}{t+\alpha}\right]^{p}$, over the $n^{\prime}$ th spatial moment $I_{n 0}(t)$ of the flux distribution. In other words,

$$
I_{n 0}^{p+n+\frac{1}{2}}=\int_{0}^{1} d t\left[\frac{(1+\alpha) t}{t+\alpha}\right]^{p} I_{n 0}(t)=\int_{0}^{1} d t\left[\frac{(1+\alpha) t}{t+\alpha}\right]^{p} \int_{-1}^{1} d x x^{n} I_{0}(t, x),
$$

where $I_{0}(t, x) d t$ is the flux of electrons having residual ranges between $t$ and $t+d t$ crossing a small spherical probe of unit cross sectional area at a distance $x$ from the source. The different columns in table 2 correspond to fixed values of $p$. Enough $p$ values have been included to make possible calculations of electron spectra by reconstruction from these "residual range moments". Similarly, the $I_{n 0}^{m}$ can be combined with new representations of the stopping power of the type given by (14) to yield improved calculations of energy dissipation distributions, or perhaps calculations, using the stopping power of air, which give results corresponding to measurements of air ionization in a cavity chamber. Note that each number in table 2 ends in a symbol $E-n$ which stands for $\times 10^{-n}$; thus $0.1000000-01$, is to be interpreted as $0.1 \times 10^{-1}$, or 0.01 .

Two sets of curves are given to illustrate data in table 1 . In figure 1 curves are drawn of the energy dissipation distributions for plane perpendicular sources in copper. Each curve corresponds to

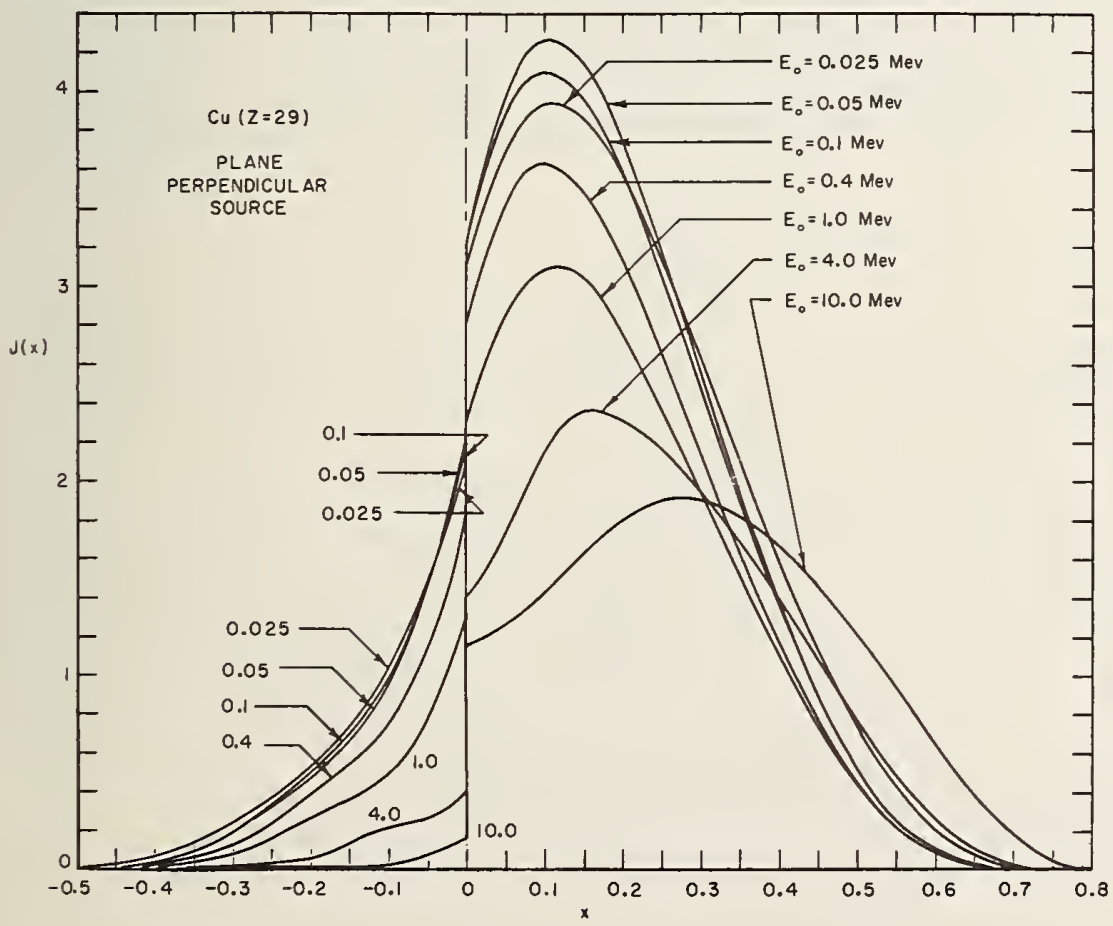

FIGURE 1. Energy dissipation distribution for plane perpendicular sources of different initial energies in copper.

The irregularities for negative $x$ are spurious effects arising from inadequacy of the representation. 
FIGLRE 2. Energy dissipation distribution for point isotropic sources of initial energy $E_{0}=0.7$. Mev in different materials.

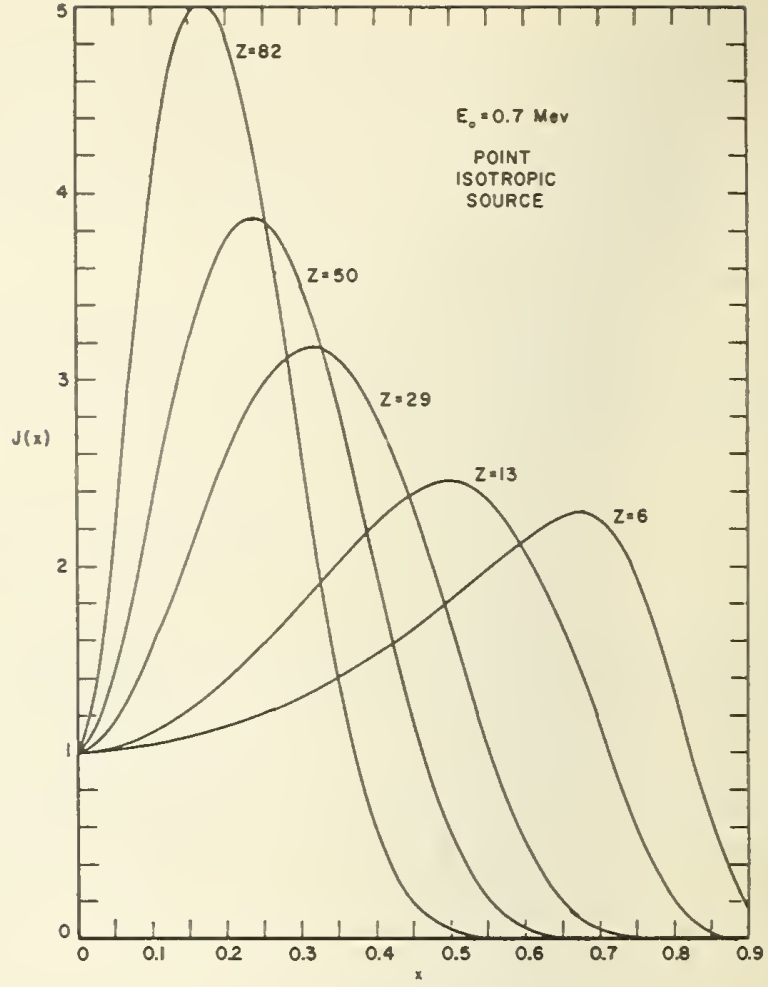

a different source energy. The curves show marked similarity for nonrelativistic source energies, which illustrates the scaling with range. They also show the tendency at relativistic energies for electrons to dissipate their energy farther out from the source, more in the manner of protons and $\alpha$ particles. Figure 2 gires distributions $J(x)$ for point isotropic sources corresponding to different $Z$ values, for an initial energy $E_{0}=0.7 \mathrm{Mev}$. These curres show clearly the trend towards greater energy dissipation near the source for larger $Z$ values.

\subsection{Interpolation}

Three types of interpolation are of interest, namely interpolation in $x$, interpolation in $E_{0}$, and interpolation in $Z$.

The fairly fine mesh in $x$, namely $\Delta x=0.025$, should make possible an interpolation everywhere of precision comparable with the number of digits tabulated, although a quadratic, rather than linear, interpolation formula may be required.

Interpolation in source energy $E_{0}$ is a fairly simple matter orer most of the $\left(E_{0}, x\right)$ region covered by the tabulations. This is because the curves have a strong tendency to preserve their shape through source energy changes. Because logarithmically spaced source energies have been used, interpolation should be based on the rariable $\log E_{0}$ rather than $E_{0}$. For energies between $4 \mathrm{Mer}$ and $10 \mathrm{Mev}$, and for large $x$ ralues, there occur substantial shape changes which require a more sophisticated interpolation procedure which takes into account the analytic form of the approximating functions. Thus, for example, it should be substantially more accurate to interpolate the function $\{1 / A \ln J(x)+x /(1-x)\}$, if a power series representation is used for the interpolation, even though the ralue of $J(x)$ must then be obtained from a difference between terms which affect the ralue of $J(x)$ exponentially.

Interpolation in $Z$ is more difficult because of progressive shape changes with $Z$ which result in great changes from one curve to the next. Accurate interpolation will therefore often require that the analytic form (22) be taken into account. This can be done in the manner already mentioned, by interpolating a well-chosen function of $J(x)$, such as $\{1 / A \ln J(x)+x /(1-x)\}$, rather than $J(x)$ itself. The value of $J(x)$ can be obtained from this function utilizing a value for the constant $A$ which is also interpolated from the $A^{\text {PTI }}$ or $A^{\text {PLP }}$ values of table 1 . 
Of special interest are interpolations for compounds and mixtures. Here it should be noted that the single, most important, determining piece of input data is the $S_{1}(1)$, which is nearly proportional to $Z+1$. One can therefore average $Z$ over the constituent elements in the compound and use this value in an interpolation of the type discussed in the preceding paragraph.

Interpolation can be used to modify the results presented here to correspond to more accurate range and stopping power data; but changes of this type will almost certainly be small because they affect the calculations chiefly through the factor $r_{0}$ in the $S_{l}$ values. A 3-percent change in $r_{0}$ would have about the same effect on the calculations as a 3 -percent change in the atomic number. A similar change in the stopping power at all electron energies would have no effect because of the scaling which is utilized in de-dimensionalizing $J(x)$. Thus, new range and stopping power values can replace those presented in table 1 for most purposes, without further modifications in the results.

It should be mentioned that in a number of cases solutions were accepted for which one of the $\beta_{i}$ exceeded unity by a small amount, say 2 percent. Because of this, the extreme asymptotic values of $J(x)$, i.e., for $x \approx 1$, do not always follow closely the trend of $(22)$. This is noticeable mostly for the low $Z$ elements. Interpolation will be less accurate when this occurs, but the values of $J(x)$ involved are small enough and inaccurate enough for other reasons that this should not matter.

\subsection{Comparisons With Experimental Data}

A number of experiments are available for comparison with the data presented here, and fortunately they are of high quality. By and large; the values in table 1 agree with the results recorded in [1], which in turn compared well with experiments due to Frantz using monoenergetic electrons incident perpendicularly on beryllium, aluminum, and gold [9]. Other results of [1] proved to be in good agreement with experiments using $P^{32}$ beta ray sources in air (Clark, Brar, and Marinelli, point isotropic source geometry) [10], and in polystyrene (Loevinger, [11] plane isotropic source geometry).

Since the publication of [1], further experiments and calculations have been made by Huffman [12]. Huffman's results pertain to a monoenergetic electron beam incident perpendicularly on aluminum, with measurement of the energy dissipated in plane layers of air. His source energies are all in the proximity of $0.1 \mathrm{Mev}$. Huffman performed calculations, using the simplified method given in [1] which assumes $\alpha$ to be infinite. His theoretical values were in fair agreement with his experiments.

A fine summary of all this work is given in the Encyclopedia of Physics, vol. 34, in a review article by Birkhoff [13].

Figure 3. Comparison of experimental data [12] with energy dissipation data from table 1.

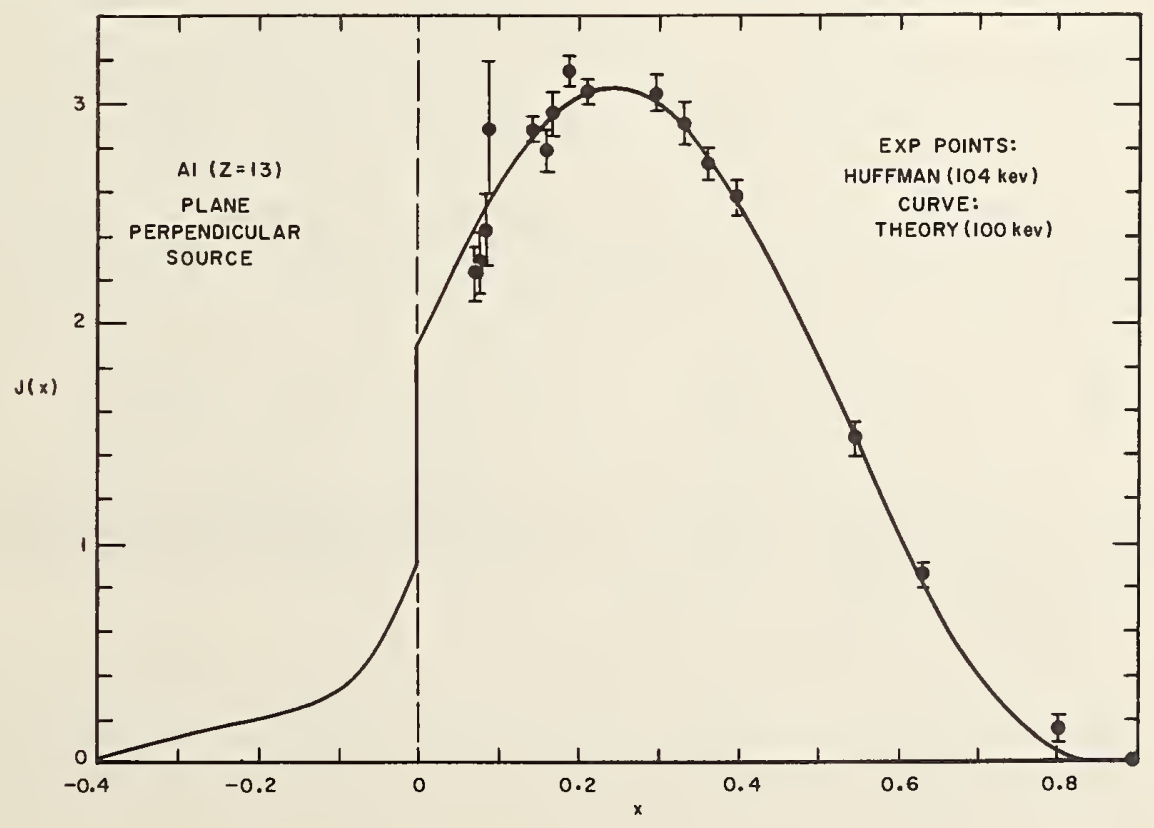




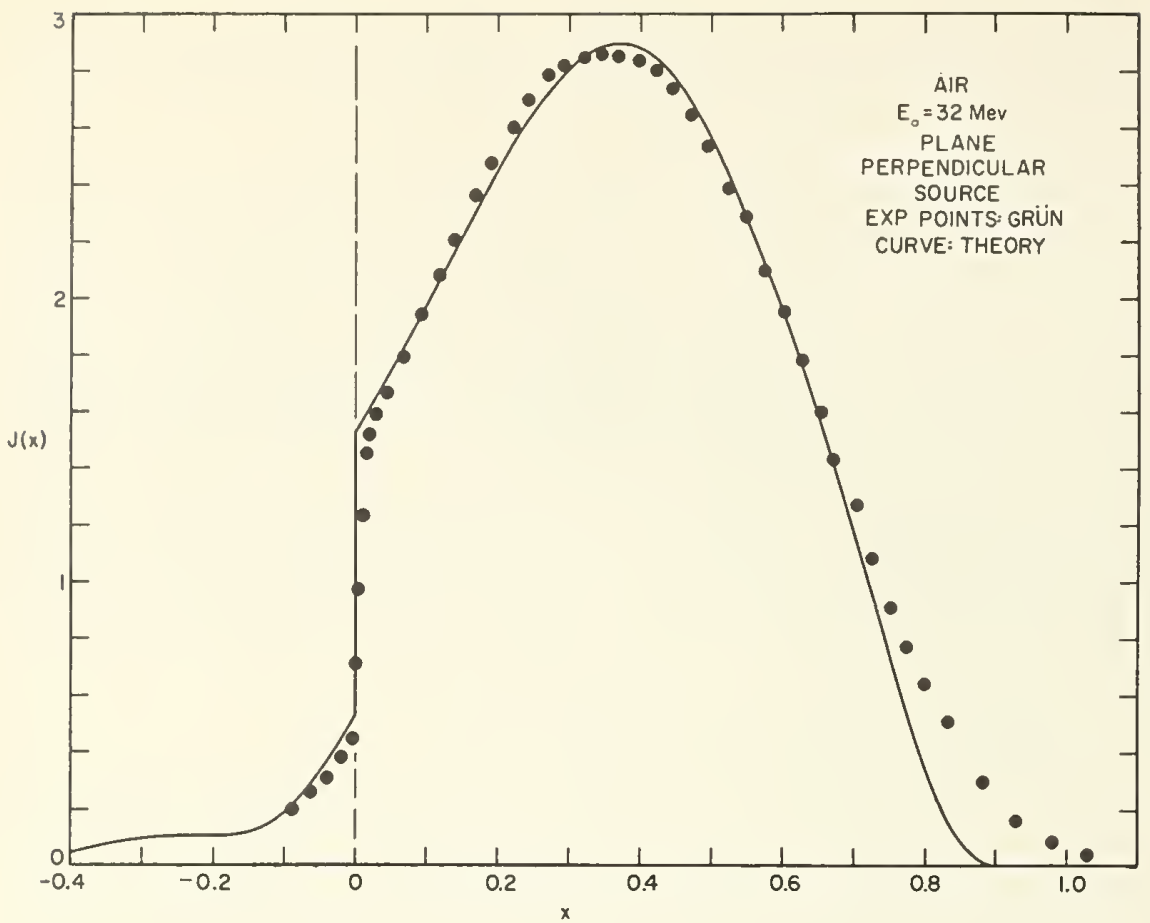

FIGURE 4. Comparison of experimental results of Grün [14] with theoretical data interpolated from table 1 .

Although the data presented in this Monograph relate to energy dissipated in the material, rather than in an air probe, it is instructive to compare some of these new tabular data with Huffman's experimental results for $0.1 \mathrm{Mev}$ electrons. Such a comparison is given in figure 3. The experimental results have been scaled to compare with $J(\alpha)$ by use of range and stopping power values from [1]. Agreement is seen to be very good, actually a little better than in the case of Huffman's calculations. Perhaps the use of a finite $\alpha$, which permits a slightly better representation of the energy dependence of $S(t, \theta)$ even at low energies, causes the difference between the two calculations.

The most complete experiment of this type is that of A. E. Grün, who made extremely detailed and consistent measurements of the energy dissipation distribution of rather low energy electrons $(0.005$ to $0.054 \mathrm{Mev})$ in air [14]. Grün determined the energy dissipation in plane layers of air perpendicular to a beam source, and his values include positions behind the source plane. In fact, Grün's experiments exhibit with remarkable clarity the discontinuity at the source plane, and he uses this feature as an experimental determination of the stopping power of electrons at these (low) source energies.

Results from table 1 have been interpolated to obtain a comparison with Grün's curve for 0.032 $\mathrm{Mev}$, and this is shown in figure 4. Both curves are absolute, having been normalized to unit electron source strength, so that the discontinuity measures the stopping power in $\mathrm{Mev}-\mathrm{cm}^{2} / \mathrm{g}$. Substantial disagreement occurs only in two areas. One is at large penetrations, where the experimental curve is high due to the straggling in range which has been omitted from the calculations. The other is for penetrations behind the source, where the theoretical representation breaks down due to the calculation of small differences between even and odd component distributions.

Comparisons at higher energies $(\sim 2 \mathrm{Mev})$ in aluminum have been made with earlier work by Trump, Wright, and Clark [15] and indicate fairly good agreement. In high- $Z$ materials, at both high and low energies, where one expects the schematization to break down, there do not appear to be experimental results at present.

Construction of the computer programs and applications of the computer were accomplished in collaboration with Mrs. Sally Peavy of the NBS Computer Laboratory. Calculation of input data was accomplished with the aid of R. Bach and Joel Rogers. Graphing of the computer output for the purpose of selection of suitable results was accomplished with the help of Mrs. Ann Nelms. Many people aided this project in other ways. 
Table 1. Energy Dissipation Function, $J(x)$, and Input Data

CARBON

$Z=6, E_{0}=0.025 \mathrm{Mev}$

STOPPING POWER

$$
(\mathrm{dE} / \mathrm{dr})_{E_{0}}=9.97 \mathrm{Mev}\left(\mathrm{cm}^{2} / \mathrm{g}\right)
$$

POINT PLANE

ISOTROPIC PERPENDICULAR SOURCE SOURCE

RESIDUAL RANGE

$$
r_{0}=0.00141 \mathrm{~g} / \mathrm{cm}^{2}
$$

OTHER PARAMETERS

$$
\begin{array}{ll}
d_{1}=1.080 & A_{1}=0.8079 \\
{ }=-107.8 & A_{2}=0.2708 \\
{ }_{A} \text { PTI }=0.7751 & A_{3}=0.0380 \\
{ }_{A} \text { PLP }=0.9124 & A_{4}=-0.1167
\end{array}
$$

SCATTERING COEFFICIENTS

$\begin{array}{cccc}\theta & \sigma / \sigma_{\mathrm{R}} & l & \mathrm{~S} \ell \\ 15^{\circ} & 1.004 & 1 & 1.090 \\ 30 & 1.003 & 2 & 2.770 \\ 45 & 0.998 & 3 & 4.867 \\ 60 & 0.990 & 4 & 7.272 \\ 75 & 0.979 & 5 & 9.977 \\ 90 & 0.966 & 6 & 12.87 \\ 105 & 0.953 & 7 & 15.90 \\ 120 & 0.940 & 8 & 19.04 \\ 135 & 0.928 & 9 & 22.68 \\ 150 & 0.920 & 10 & 26.43 \\ 165 & 0.914 & 11 & 30.26 \\ 180 & 0.912 & 12 & 34.16\end{array}$

STOPPING POWER

$$
(\mathrm{dE} / \mathrm{dr})_{E_{O}}=5.95 \mathrm{Mev}\left(\mathrm{cm}^{2} / \mathrm{g}\right)
$$

RESIDUAL RANGE

$$
r_{0}=0.00479 \mathrm{~g} / \mathrm{cm}^{2}
$$

OTHER PARAMETERS

$$
\begin{array}{ll}
\mathrm{d}_{1}=1.115 & \mathrm{~A}_{1}=0.7176 \\
\alpha=12.76 & A_{2}=1.438 \\
{ }_{A} \mathrm{PTI}=0.7913 & A_{3}=-2.387 \\
{ }_{A} \mathrm{PLP}=0.9343 & A_{4}=1.231
\end{array}
$$

SCATTERING COEFFICIENTS

$\begin{array}{cccc}\theta & \sigma \not R_{R} & l & s \ell \\ 15^{\circ} & 1.004 & 1 & 1.034 \\ 30 & 1.001 & 2 & 2.687 \\ 45 & 0.990 & 3 & 4.800 \\ 60 & 0.974 & 4 & 7.274 \\ 75 & 0.953 & 5 & 10.04 \\ 90 & 0.930 & 6 & 13.04 \\ 105 & 0.905 & 7 & 16.23 \\ 120 & 0.882 & 8 & 19.58 \\ 135 & 0.862 & 9 & 23.06 \\ 150 & 0.846 & 10 & 26.64 \\ 165 & 0.836 & 11 & 30.29 \\ 180 & 0.833 & 12 & 34.00\end{array}$

$\mathrm{J}(\mathrm{x}) \quad \mathrm{J}(\mathrm{x}) \quad \mathrm{J}(-\mathrm{x})$

$x$
0.000
0.025
0.050
0.075
0.100
0.125
0.150
0.175
0.200
0.225
0.250
0.275
0.300
0.325
0.350
0.375
0.400
0.425
0.450
0.475

\section{J(x)}

1.000

1.016

1.041

1.519

1.615

1.072

1.713

0.422

0.341

0.266

0.200

\subsection{1}

\subsection{8}

1.812

1. 211

1. 914

0.144

1.272

2.022

0.103

0.081

\subsection{0}

2.242

1.415

1.497

1.586

2.346

0.077

$\begin{array}{ll}1.682 & 2.615\end{array}$

1.785

1.893

2.008

2.685

2.742

2.783

0.078

$2.127 \quad 2.806$

2.251

2.806
2.808
2.787
2.741

2.508

0.079

0.078

0.076

0.072

0.066

0.058

0.048

0.036

0.022

0.007

$$
Z=6, E_{0}=0.05 \mathrm{Mev}
$$

$\begin{array}{cc}\text { POINT } & \text { PLANE } \\ \text { ISOTROPIC } & \text { PERPENDICULAR } \\ \text { SOURCE } & \text { SOURCE }\end{array}$

$\begin{array}{cccc}\mathbf{x} & \mathrm{J}(\mathrm{x}) & \mathrm{J}(\mathrm{x}) & \mathrm{J}(-\mathrm{x}) \\ 0.000 & 1.000 & 1.363 & 0.363 \\ 0.025 & 1.008 & 1.443 & 0.290 \\ 0.050 & 1.024 & 1.526 & 0.224 \\ 0.075 & 1.049 & 1.615 & 0.167\end{array}$

0.075

0.100

1.082

1.713

0.123

0.125

1.123

1.825

0.150

1.172

1.950

0.096

0.175

1.230

2.075

0.086

0.200

0.225

1.296

2.195

1. 369

2.310

0.250

1.451

2.418

0.076

0.275

1.540

2. 518

0.071

1.637

2.609

0.300

0.325

1.74

0.350
0.375

1.854

2.688

1.973

2.754

2.805

0.066

0.400

0.425

2.099

0.450

2.231

2.837

2.368

2.847

0.475
2.834

2.793
0.056

0.050

0.044

0.038

0.032

0.026

0.019

0.012

0.925

0.950

0.975

x

0.525

0.550

0.575

0.600

0.625

0.650

0.675

0.700

0.725

0.750

0.775

0.800

0.825

0.850

0.875

0.900

0.925

0.950

0.975
POINT

ISOTROPIC PERPENDICULAR SOURCE SOURCE

$\mathrm{J}(\mathrm{x}) \mathrm{J}(\mathrm{x}) \quad \mathrm{J}(-\mathrm{x})$

2.636

2.763

2.671

2.580

2.472

$-0.009$

2.883

2.350

$-0.012$

2.994

3.161

2.216

$-0.013$

3.203

1.910

1.735

$-0.003$

0.002

0.006

3.025

1.543

0.008

2.813

1.334

0.008

2.496

1.872

0.007

2.064

0.634

1.523

0.409

0.004

0.925

0.397

0.085

0.001

0.091

0.019

0.007

0.001

$-0.001$

0.000

0.000

$-0.000$

0.000

0.000

$-0.000$

$\begin{array}{cc}\text { POINT } & \text { PLANE } \\ \text { ISOTROPIC } & \text { PERPENDICULAR } \\ \text { SOURCE } & \text { SOURCE }\end{array}$

$\begin{array}{rrr}J(x) & J(x) & J(-x) \\ 2.654 & 2.725 & 0.005 \\ 2.798 & 2.630 & -0.001 \\ 2.939 & 2.513 & -0.005 \\ 3.072 & 2.378 & -0.008\end{array}$

$\begin{array}{lll}3.189 & 2.229 & -0.007\end{array}$

$\begin{array}{lll}3.325 & 1.888 & 0.000\end{array}$

$\begin{array}{lll}3.303 & 1.696 & 0.004\end{array}$

$\begin{array}{lll}3.185 & 1.490 & 0.007\end{array}$

$2.948 \quad 1.270 \quad 0.008$

$\begin{array}{lll}2.600 & 1.039 & 0.007\end{array}$

$\begin{array}{lll}2.174 & 0.803 & 0.004\end{array}$

$\begin{array}{lll}1.715 & 0.571 & 0.001\end{array}$

$\begin{array}{lll}1.255 & 0.358 & -0.002\end{array}$

$\begin{array}{lll}0.819 & 0.184 & -0.003\end{array}$

$\begin{array}{llll}0.446 & 0.068 & -0.003\end{array}$

$\begin{array}{llll}0.178 & 0.014 & -0.001\end{array}$

$\begin{array}{llll}0.039 & 0.001 & -0.000\end{array}$

$\begin{array}{lll}0.003 & 0.000 \quad-0.000\end{array}$

$0.000 \quad 0.000 \quad-0.000$ 


$$
\mathrm{Z}=6, \mathrm{E}_{\mathrm{o}}=0.1 \mathrm{Mev}
$$

STOPPING POWER

$$
(\mathrm{dE} / \mathrm{dr})_{\mathrm{E}_{\mathrm{O}}}=3.71 \mathrm{Mev}\left(\mathrm{cm}^{2} / \mathrm{g}\right)
$$

RESIDUAL RANGE

$$
r_{0}=0.0159 \mathrm{~g} / \mathrm{cm}^{2}
$$

OTHER PARAMETERS

$$
\begin{array}{ll}
d_{1}=1.042 & A_{1}=0.7075 \\
a^{P}=39.12 & A_{2}=0.8655 \\
A^{P T I}=0.7714 & A_{3}=-1.031 \\
A^{\text {PLP }}=0.9168 & A_{4}=0.4576
\end{array}
$$

SCATTERING COEFFICIENTS

$\begin{array}{cccc}\theta & \sigma / \sigma_{\mathrm{R}} & \ell & S \ell \\ 15^{\circ} & 1.004 & 1 & 1.016 \\ 30 & 0.996 & 2 & 2.702 \\ 45 & 0.976 & 3 & 4.908 \\ 60 & 0.946 & 4 & 7.542 \\ 75 & 0.909 & 5 & 10.54 \\ 90 & 0.868 & 6 & 13.84 \\ 105 & 0.826 & 7 & 17.42 \\ 120 & 0.786 & 8 & 21.23 \\ 135 & 0.751 & 9 & 25.24 \\ 150 & 0.725 & 10 & 29.43 \\ 165 & 0.708 & 11 & 33.78 \\ 180 & 0.702 & 12 & 38.26\end{array}$

STOPPING POWER

$$
(\mathrm{dE} / \mathrm{dr})_{\mathrm{E}_{\mathrm{O}}}=2.52 \mathrm{Mev}\left(\mathrm{cm}^{2} / \mathrm{g}\right)
$$

RESIDUAL RANGE

$$
r_{O}=0.0498 \mathrm{~g} / \mathrm{cm}^{2}
$$

OTHER PARAMETERS

$$
\begin{array}{ll}
d_{1}=1.033 & A_{1}=0.6437 \\
\alpha=18.89 & A_{2}=0.8923 \\
A^{\text {PTI }}=0.7726 & A_{3}=-0.9961 \\
A^{\text {PLP }}=0.9216 & A_{4}=0.4600
\end{array}
$$

\section{SCATTERING COEFFICIENTS}

$\begin{array}{cccc}\theta & \sigma / \sigma_{\mathrm{R}} & l & \mathrm{~S} \ell \\ 15^{\circ} & 1.003 & 1 & 0.9815 \\ 30 & 0.987 & 2 & 2.666 \\ 45 & 0.954 & 3 & 4.912 \\ 60 & 0.906 & 4 & 7.634 \\ 75 & 0.846 & 5 & 10.77 \\ 90 & 0.781 & 6 & 14.28 \\ 105 & 0.714 & 7 & 18.11 \\ 120 & 0.651 & 8 & 22.24 \\ 135 & 0.596 & 9 & 26.64 \\ 150 & 0.554 & 10 & 31.28 \\ 165 & 0.527 & 11 & 36.14 \\ 180 & 0.518 & 12 & 41.19\end{array}$

\section{POINT PLANE ISOTROPIC PERPENDICULAR SOURCE SOURCE}

$\begin{array}{clllc}x & J(x) & J(x) & J(-x) & x \\ 0.000 & 1.000 & 1.341 & 0.341 & 0.500 \\ 0.025 & 1.011 & 1.424 & 0.270 & 0.525 \\ 0.050 & 1.029 & 1.508 & 0.204 & 0.550 \\ 0.075 & 1.054 & 1.595 & 0.145 & 0.575 \\ 0.100 & 1.086 & 1.687 & 0.097 & 0.600 \\ 0.125 & 1.124 & 1.786 & 0.062 & 0.625 \\ 0.150 & 1.169 & 1.895 & 0.045 & 0.650 \\ 0.175 & 1.221 & 2.013 & 0.045 & 0.675 \\ & & & & \\ 0.200 & 1.279 & 2.130 & 0.050 & 0.700 \\ 0.225 & 1.344 & 2.241 & 0.053 & 0.725 \\ 0.250 & 1.415 & 2.345 & 0.056 & 0.750 \\ 0.275 & 1.493 & 2.442 & 0.058 & 0.775 \\ & & & & \\ 0.300 & 1.578 & 2.528 & 0.058 & 0.800 \\ 0.325 & 1.669 & 2.603 & 0.056 & 0.825 \\ 0.350 & 1.767 & 2.664 & 0.052 & 0.850 \\ 0.375 & 1.871 & 2.708 & 0.046 & 0.875 \\ 0.400 & 1.981 & 2.734 & 0.038 & 0.900 \\ 0.425 & 2.097 & 2.737 & 0.027 & 0.925 \\ 0.450 & 2.218 & 2.716 & 0.014 & 0.950 \\ 0.475 & 2.344 & 2.669 & 0.001 & 0.975\end{array}$$$
Z=6, E_{0}=0.2 \mathrm{Mev}
$$

$\begin{array}{cc}\text { POINT } & \text { PLANE } \\ \text { ISOTROPIC } & \text { PERPENDICULAR } \\ \text { SOURCE } & \text { SOURCE }\end{array}$

$\begin{array}{ccccc}x & J(x) & J(x) & J(-x) & x \\ 0.000 & 1.000 & 1.294 & 0.294 & 0.500 \\ 0.025 & 1.008 & 1.368 & 0.228 & 0.525 \\ 0.050 & 1.022 & 1.444 & 0.168 & 0.550 \\ 0.075 & 1.042 & 1.522 & 0.115 & 0.575 \\ & & & & \\ 0.100 & 1.068 & 1.606 & 0.071 & 0.600 \\ 0.125 & 1.100 & 1.698 & 0.041 & 0.625 \\ 0.150 & 1.138 & 1.800 & 0.027 & 0.650 \\ 0.175 & 1.181 & 1.911 & 0.029 & 0.675 \\ 0.200 & 1.231 & 2.022 & 0.035 & 0.700 \\ 0.225 & 1.286 & 2.126 & 0.040 & 0.725 \\ 0.250 & 1.347 & 2.224 & 0.044 & 0.750 \\ 0.275 & 1.414 & 2.314 & 0.047 & 0.775 \\ & & & & \\ 0.300 & 1.488 & 2.395 & 0.048 & 0.800 \\ 0.325 & 1.567 & 2.463 & 0.048 & 0.825 \\ 0.350 & 1.653 & 2.518 & 0.045 & 0.850 \\ 0.375 & 1.745 & 2.557 & 0.040 & 0.875 \\ 0.400 & 1.843 & 2.577 & 0.032 & 0.900 \\ 0.425 & 1.948 & 2.576 & 0.021 & 0.925 \\ 0.450 & 2.059 & 2.551 & 0.009 & 0.950 \\ 0.475 & 2.176 & 2.503 & -0.003 & 0.975 \\ & & & & \end{array}$

POINT ISOTROPIC PERPENDICULAR SOURCE
PLANE SOURCE
$\mathrm{J}(\mathrm{x}) \mathrm{J}(\mathrm{x}) \quad \mathrm{J}(-\mathrm{x})$

2.474

2.605

2.736

2.864

$$
2.599
$$

2.507

2. 399

2.278

$-0.009$

$-0.005$

$-0.014$

2.9832 .145

3.983

3.084

2.145

3.154

3.174

1.844

1.671

$3.116 \quad 1.481$

$2.952 \quad 1.275$

$2.667 \quad 1.054$

$2.266 \quad 0.824$

1.782

1.276

0.822

0.465

0.593

0.377

0.197

0.074

0.218

0.076

0.015

0.001

0.016

0.001
0.000

0.000
$-0.004$

0.001

0.005

0.007

0.008

0.008

0.006

0.003

0.000

$-0.002$

$-0.003$

$-0.003$

$-0.001$

$-0.000$

0.000

0.000

POINT, PLANE

ISOTROPIC PERPENDICULAR SOURCE SOURCE

$\mathrm{J}(\mathrm{x}) \mathrm{J}(\mathrm{x}) \mathrm{J}(-\mathrm{x})$

$2.298 \quad 2.433 \quad-0.012$

$2.425 \quad 2.344 \quad-0.016$

$2.552 \quad 2.242 \quad-0.013$

$\begin{array}{lll}2.676 & 2.128 & -0.008\end{array}$

$2.789 \quad 2.004 \quad-0.003$

$\begin{array}{lll}2.878 & 1.869 & 0.001\end{array}$

$2.929 \quad 1.721 \quad 0.004$

$\begin{array}{lll}2.928 & 1.558 & 0.006\end{array}$

$\begin{array}{lll}2.861 & 1.380 & 0.007\end{array}$

$2.714 \quad 1.186 \quad 0.007$

$\begin{array}{lll}2.472 & 0.978 & 0.005\end{array}$

$\begin{array}{lll}2.132 & 0.762 & 0.003\end{array}$

$\begin{array}{lll}1.702 & 0.545 & 0.000\end{array}$

$\begin{array}{llll}1.220 & 0.344 & -0.002\end{array}$

$\begin{array}{llll}0.753 & 0.178 & -0.003\end{array}$

$\begin{array}{llll}0.387 & 0.066 & -0.002\end{array}$

$\begin{array}{lll}0.171 & 0.013 & -0.001\end{array}$

$\begin{array}{llll}0.071 & 0.001 & -0.000\end{array}$

$\begin{array}{llll}0.027 & 0.000 & -0.000\end{array}$

$\begin{array}{llll}0.009 & 0.000 & -0.000\end{array}$ 
STOPPING PO'NER

$$
(\mathrm{dE} / \mathrm{dr})_{\mathrm{E}_{0}}=1.92 \mathrm{Mer}\left(\mathrm{cm}^{2} / \mathrm{g}\right)
$$

RESIDUAL RANGE

$$
r_{0}=0.143 \mathrm{~g} / \mathrm{cm}^{2}
$$

OTHER PARAMETERS

$$
\begin{array}{ll}
d_{1}=1.016 & A_{1}=0.5417 \\
a=9.779 & A_{2}=0.9422 \\
A^{\text {PTI }}=0.7603 & A_{3}=0.8166 \\
A^{\text {PLP }}=0.9091 & A_{4}=0.3327
\end{array}
$$

SCATTERING COEFFICIENTS

$\begin{array}{cccc}\theta & \sigma / \sigma_{\mathrm{R}} & l & \mathrm{~s}_{l} \\ 15^{\circ} & 1.002 & 1 & 0.9221 \\ 30 & 0.9977 & 2 & 2.555 \\ 45 & 0.929 & 3 & 4.766 \\ 60 & 0.860 & 4 & 7.481 \\ 75 & 0.776 & 5 & 10.64 \\ 90 & 0.683 & 6 & 14.22 \\ 105 & 0.590 & 7 & 18.16 \\ 120 & 0.501 & 8 & 22.44 \\ 135 & 0.425 & 9 & 27.04 \\ 150 & 0.366 & 10 & 31.94 \\ 165 & 0.328 & 11 & 37.10 \\ 180 & 0.316 & 12 & 42.51\end{array}$

STOPPING POWER

$$
(d E / d r)_{E_{0}}=1.71 \mathrm{Mev}\left(\mathrm{cm}^{2} / \mathrm{g}\right)
$$

RESIDUAL RANGE

$$
r_{0}=0.311 \mathrm{~g} / \mathrm{cm}^{2}
$$

OTHER PARAMETERS

$$
d_{1}=1.004 \quad A_{1}=0.4643
$$$$
a=5.157 \quad A_{2}=0.9142
$$$$
A^{\text {PTI }}=0.7383 \quad A_{3}=-0.5978
$$$$
{ }_{A}^{P L P}=0.8842 \quad A_{4}=0.2193
$$

SCATTERTNG COEFFICIENTS

$\begin{array}{cccc}\theta & \sigma / \sigma_{R} & l & s \ell \\ 15^{\circ} & 1.001 & 1 & 0.8413 \\ 30 & 0.970 & 2 & 2.362 \\ 45 & 0.911 & 3 & 4.444 \\ 60 & 0.828 & 4 & 7.022 \\ 75 & 0.728 & 5 & 10.05 \\ 90 & 0.617 & 6 & 13.50 \\ 105 & 0.505 & 7 & 17.31 \\ 120 & 0.400 & 8 & 21.50 \\ 135 & 0.308 & 9 & 26.01 \\ 150 & 0.238 & 10 & 30.84 \\ 165 & 0.194 & 11 & 35.97 \\ 180 & 0.179 & 12 & 41.39\end{array}$

CARBON

$$
Z=6, E_{0}=0.4 \mathrm{Mev}
$$

$\begin{array}{cc}\text { POINT } & \text { PLANE } \\ \text { ISOTROPIC } & \text { PERPENDICULAR } \\ \text { SOURCE } & \text { SOURCE }\end{array}$

$\begin{array}{ccccc}\mathbf{x} & \mathrm{J}(\mathrm{x}) & \mathrm{J}(\mathrm{x}) & \mathrm{J}(-\mathbf{x}) & \mathbf{x} \\ 0.000 & 1.000 & 1.233 & 0.233 & 0.500 \\ 0.025 & 1.007 & 1.296 & 0.176 & 0.525 \\ 0.050 & 1.018 & 1.362 & 0.125 & 0.550 \\ 0.075 & 1.035 & 1.430 & 0.079 & 0.575 \\ 0.100 & 1.056 & 1.503 & 0.042 & 0.600 \\ 0.125 & 1.081 & 1.584 & 0.017 & 0.625 \\ 0.150 & 1.112 & 1.674 & 0.006 & 0.650 \\ 0.175 & 1.147 & 1.774 & 0.011 & 0.675 \\ & & & & \\ 0.200 & 1.187 & 1.873 & 0.018 & 0.700 \\ 0.225 & 1.231 & 1.967 & 0.026 & 0.725 \\ 0.250 & 1.281 & 2.055 & 0.031 & 0.750 \\ 0.275 & 1.335 & 2.134 & 0.036 & 0.775 \\ 0.300 & 1.394 & 2.205 & 0.038 & 0.800 \\ 0.325 & 1.458 & 2.264 & 0.038 & 0.825 \\ 0.350 & 1.527 & 2.310 & 0.037 & 0.850 \\ 0.375 & 1.602 & 2.340 & 0.032 & 0.875 \\ & & & & \\ 0.400 & 1.681 & 2.352 & 0.025 & 0.900 \\ 0.425 & 1.766 & 2.344 & 0.016 & 0.925 \\ 0.450 & 1.855 & 2.314 & 0.004 & 0.950 \\ 0.475 & 1.949 & 2.262 & -0.006 & 0.975\end{array}$

$\mathrm{z}=6, \mathrm{E}_{\mathrm{o}}=0.7 \mathrm{Mev}$

$\begin{array}{cc}\text { POINT } & \text { PLANE } \\ \text { ISOTROPIC } & \text { PERPENDICULAR } \\ \text { SOURCE } & \text { SOURCE }\end{array}$

$x$
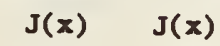

1.000

1.176

0.025

1.004

1.227

$J(-x)$

$1.013 \quad 1.279$

0.176

0.050

0.075

1.025

1.334

0.089

0.052

0.100

0.125

1.040

1.394

0.022

0.150

1.060

1.462

0.022

1.083

$1.539-0.006$

0.175

1.110

$\begin{array}{lll}1.625 & -0.001\end{array}$

0.200

0.225

$\begin{array}{lll}1.140 & 1.712 & 0.007\end{array}$

0.250

1.174

1.795

1.213

1.872

0.014

0.020

0.275

0.300

1.25

1.942

0.025

\section{$\begin{array}{lll}1.301 & 2.004 & 0.029\end{array}$}

0.350

0.030

1.405

2.097

0.030

0.375

0.400

0.425

0.450

0.475 $\begin{array}{lll}1.463 & 2.123 & 0.027\end{array}$

$\begin{array}{lll}1.525 & 2.134 & 0.021\end{array}$

1.591

$$
1.661
$$

2.126

0.013

1.735 $\begin{array}{rr}2.098 & 0.003 \\ 2.050 & -0.007\end{array}$
POINT PLANE

ISOTROPIC PERPENDICULAR SOURCE

SOURCE

$\mathrm{J}(\mathrm{x}) \quad \mathrm{J}(\mathrm{x}) \quad \mathrm{J}(-\mathrm{x})$

$\begin{array}{lll}2.048 & 2.192 & -0.014\end{array}$

$\begin{array}{lll}2.150 & 2.107 & -0.015\end{array}$

$\begin{array}{lll}2.254 & 2.012 & -0.012\end{array}$

$\begin{array}{lll}2.358 & 1.905 & -0.007\end{array}$

$\begin{array}{lll}2.457 & 1.792 & -0.003\end{array}$

$\begin{array}{lll}2.543 & 1.670 & 0.001\end{array}$

$\begin{array}{lll}2.601 & 1.536 & 0.004\end{array}$

$\begin{array}{lll}2.611 & 1.390 & 0.005\end{array}$

$\begin{array}{lll}2.544 & 1.231 & 0.006\end{array}$

$\begin{array}{lll}2.380 & 1.058 & 0.006\end{array}$

$\begin{array}{lll}2.126 & 0.873 & 0.005\end{array}$

$\begin{array}{lll}1.813 & 0.681 & 0.002\end{array}$

$\begin{array}{lll}1.466 & 0.488 & 0.000\end{array}$

$\begin{array}{lll}1.098 & 0.307 & -0.002\end{array}$

$\begin{array}{lll}0.731 & 0.160 & -0.003\end{array}$

$\begin{array}{lll}0.401 & 0.060 & -0.002\end{array}$

$\begin{array}{lll}0.157 & 0.012 & -0.001\end{array}$

$\begin{array}{lll}0.031 & 0.001 & -0.000\end{array}$

$\begin{array}{lll}0.001 & 0.000 & -0.000\end{array}$

$\begin{array}{lll}0.000 & 0.000 & -0.000\end{array}$
POINT PLANE

ISOTROPIC PERPENDICULAR SOURCE SOURCE

$J(x) \quad J(x) \quad J(-x)$

0.500

0.525

0.550

0.575

0.600

0.625

0.650

0.675

1.813

1.894

1.985

1.907

1.977

1.819

$-0.013$

2.060

1.724

2.141

1.623

2.213

1.513

2.268

1.394

$-0.003$

2.288

1.265

0.003

0.700

2.253

1.123

0.725

0.750

2.137

0.970

1.929

0.805

0.775

1.642

0.632

0.004

0.002

0.800

1.306

0.458

0.000

0.825

0.963

0.293

$-0.002$

0.850

0.644

0.155

$-0.003$

0.372

0.059

0.900

0.168

0.012

0.049

0.001

$-0.001$

0.950

0.006

0.000

$-0.000$

0.975

0.000

0.000

$-0.000$ 
Table 1. Energy Dissipation Function, $J(x)$, and Input Data - Continued

CARBON

$$
z=6, E_{0}=1.00 \mathrm{Mev}
$$

STOPPING POWER

$$
(\mathrm{dE} / \mathrm{dr})_{E_{O}}=1.64 \mathrm{Nev}\left(\mathrm{cm}^{2} / \mathrm{g}\right)
$$

RESIDUAL RANGE

$$
r_{0}=0.491 \mathrm{~g} / \mathrm{cm}^{2}
$$

\section{OTHER FARAMETERS}

$$
\begin{array}{ll}
d_{1}=0.9994 & A_{1}=0.4211 \\
\alpha=3.373 & A_{2}=0.8811 \\
{ }_{A}{ }^{P T I}=0.7135 & A_{3}=-0.4549 \\
A^{\text {PLP }}=0.8553 & A_{4}=0.1527
\end{array}
$$

SCATTERING COEFFICIENTS

\begin{tabular}{cccc}
$\theta$ & $\sigma \not p$ & $l$ & \multicolumn{1}{c}{$S_{\ell}$} \\
$15^{\circ}$ & 1.000 & 1 & 0.7709 \\
30 & 0.967 & 2 & 2.171 \\
45 & 0.903 & 3 & 4.104 \\
60 & 0.814 & 4 & 6.510 \\
75 & 0.705 & 5 & 9.348 \\
90 & 0.586 & 6 & 12.59 \\
105 & 0.456 & 7 & 16.20 \\
120 & 0.352 & 8 & 20.16 \\
135 & 0.254 & 9 & 24.45 \\
150 & 0.179 & 10 & 29.06 \\
165 & 0.131 & 11 & 33.96 \\
180 & 0.115 & 12 & 39.15
\end{tabular}

STOPPING POWER

$$
(\mathrm{dE} / \mathrm{dr})_{E_{0}}=1.62 \mathrm{Mev}\left(\mathrm{cm}^{2} / \mathrm{g}\right)
$$

RESIDUAL RANGE

$$
r_{0}=1.11 \mathrm{~g} / \mathrm{cm}^{2}
$$

OTHER PARAMETERS

$$
\begin{array}{ll}
d_{1}=1.021 & A_{1}=0.3432 \\
\alpha=1.365 & A_{2}=0.9489 \\
A^{\text {PTI }}=0.6539 & A_{3}=-0.4613 \\
A^{\text {PLP }}=0.7815 & A_{4}=0.1692
\end{array}
$$

\section{SCATTERING COEFEICIENTS}

\begin{tabular}{clcl}
$\theta$ & $\sigma / \sigma_{\mathrm{R}}$ & $l$ & \multicolumn{1}{c}{$\mathrm{s}_{\ell}$} \\
$15^{\circ}$ & 1.000 & 1 & 0.5895 \\
30 & 0.963 & 2 & 1.681 \\
45 & 0.894 & 3 & 3.200 \\
60 & 0.797 & 4 & 5.107 \\
75 & 0.680 & 5 & 7.373 \\
90 & 0.551 & 6 & 9.976 \\
105 & 0.421 & 7 & 12.90 \\
120 & 0.298 & 8 & 16.12 \\
135 & 0.192 & 9 & 19.63 \\
150 & 0.111 & 10 & 23.42 \\
165 & 0.059 & 11 & 27.48 \\
180 & 0.042 & 12 & 31.79
\end{tabular}

$\begin{array}{cc}\text { POINT } & \text { PLANE } \\ \text { ISOTROPIC } & \text { PERPENDICULAR } \\ \text { SOURCE } & \text { SOURCE }\end{array}$

$\begin{array}{cccc}x & J(x) & J(x) & J(-x) \\ 0.000 & 1.000 & 1.140 & 0.140 \\ 0.025 & 1.003 & 1.180 & 0.103 \\ 0.050 & 1.009 & 1.223 & 0.069 \\ 0.075 & 1.018 & 1.268 & 0.039 \\ & & & \\ 0.100 & 1.030 & 1.319 & 0.014 \\ 0.125 & 1.045 & 1.376 & -0.003 \\ 0.150 & 1.064 & 1.443 & -0.010 \\ 0.175 & 1.085 & 1.520 & -0.006 \\ & & & \\ 0.200 & 1.110 & 1.598 & 0.002 \\ 0.225 & 1.138 & 1.672 & 0.008 \\ 0.250 & 1.169 & 1.742 & 0.014 \\ 0.275 & 1.203 & 1.806 & 0.019 \\ 0.300 & 1.241 & 1.864 & 0.023 \\ 0.325 & 1.282 & 1.913 & 0.025 \\ 0.350 & 1.327 & 1.952 & 0.025 \\ 0.375 & 1.376 & 1.979 & 0.023 \\ 0.400 & 1.428 & 1.992 & 0.019 \\ 0.425 & 1.484 & 1.988 & 0.012 \\ 0.450 & 1.545 & 1.966 & 0.004 \\ 0.475 & 1.609 & 1.924 & 0.005\end{array}$

$$
Z=6, E_{0}=2.00 \mathrm{Mev}
$$

$\begin{array}{cc}\text { POINT } & \text { PLANE } \\ \text { ISOTROPIC } & \text { PERPENDICLLAR } \\ \text { SOURCE } & \text { SOURCE }\end{array}$

$\begin{array}{cccc}x & J(x) & J(x) & J(-x) \\ 0.000 & 1.000 & 1.064 & 0.064 \\ 0.025 & 1.001 & 1.088 & 0.042 \\ 0.050 & 1.005 & 1.114 & 0.021 \\ 0.075 & 1.011 & 1.144 & 0.005 \\ & & & \\ 0.100 & 1.020 & 1.180 & -0.006 \\ 0.125 & 1.030 & 1.225 & -0.009 \\ 0.150 & 1.041 & 1.278 & -0.003 \\ 0.175 & 1.052 & 1.332 & 0.003 \\ & & & \\ 0.200 & 1.065 & 1.385 & 0.008 \\ 0.225 & 1.079 & 1.438 & 0.012 \\ 0.250 & 1.095 & 1.491 & 0.014 \\ 0.275 & 1.174 & 1.542 & 0.015 \\ & & & \\ 0.300 & 1.134 & 1.592 & 0.013 \\ 0.325 & 1.157 & 1.639 & 0.010 \\ 0.350 & 1.183 & 1.681 & 0.007 \\ 0.375 & 1.213 & 1.715 & 0.004 \\ 0.400 & 1.247 & 1.741 & 0.002 \\ 0.425 & 1.285 & 1.755 & -0.000 \\ 0.450 & 1.328 & 1.756 & -0.002 \\ 0.475 & 1.377 & 1.741 & -0.003\end{array}$

POINT PLANE

ISOTROPIC PERPENDICULAR SOUFCE SOURCE

$\mathrm{J}(\mathrm{x}) \quad \mathrm{J}(\mathrm{x}) \quad \mathrm{J}(-\mathrm{x})$

$\begin{array}{lllll}0.500 & 1.677 & 1.866 & -0.011\end{array}$

$\begin{array}{lllll}0.525 & 1.747 & 1.795 & -0.013\end{array}$

$\begin{array}{lllll}0.550 & 1.819 & 1.714 & -0.011\end{array}$

$\begin{array}{llll}0.575 & 1.888 & 1.626 & -0.007\end{array}$

$\begin{array}{llll}0.600 & 1.949 & 1.532 & -0.003\end{array}$

$\begin{array}{llll}0.625 & 1.997 & 1.431 & -0.000\end{array}$

$\begin{array}{llll}0.650 & 2.027 & 1.322 & 0.002\end{array}$

$\begin{array}{llll}0.675 & 2.032 & 1.203 & 0.004\end{array}$

$\begin{array}{llll}0.700 & 2.001 & 1.073 & 0.065\end{array}$

$\begin{array}{llll}0.725 & 1.927 & 0.931 & 0.005\end{array}$

$\begin{array}{llll}0.750 & 1.797 & 0.778 & 0.004\end{array}$

$\begin{array}{llll}0.775 & 1.603 & 0.617 & 0.002\end{array}$

$\begin{array}{llll}0.800 & 1.339 & 0.452 & 0.000\end{array}$

$\begin{array}{llll}0.825 & 1.014 & 0.295 & -0.001\end{array}$

$\begin{array}{llll}0.850 & 0.655 & 0.160 & -0.002\end{array}$

$\begin{array}{llll}0.875 & 0.324 & 0.063 & -0.002\end{array}$

$\begin{array}{lllll}0.900 & 0.097 & 0.014 & -0.001\end{array}$

$\begin{array}{llll}0.925 & 0.010 & 0.001 & -0.000\end{array}$

$\begin{array}{lllll}0.950 & 0.000 & 0.000 & -0.000\end{array}$

$\begin{array}{llll}0.975 & 0.000 & 0.000 & -0.000\end{array}$ 
CARBON

$$
z=6, E_{0}=4.00 \mathrm{Mev}
$$

STUPPING POWER

$$
(\mathrm{dE} / \mathrm{dr})_{E_{0}}=1.67 \mathrm{Mev}\left(\mathrm{cm}^{2} / \mathrm{g}\right)
$$

RESIDUAL RANGE

$$
\begin{array}{ll}
r_{0}=2.32 \mathrm{~g} / \mathrm{cm}^{2} \\
\text { OTHER PARAMETERS } \\
d_{1}=1.041 & A_{1}=0.2978 \\
\alpha=0.6220 & A_{2}=0.9285 \\
{ }_{A} \text { PTI }=0.5522 & A_{3}=-0.3785 \\
{ }_{A} \text { PLP }=0.6549 & A_{4}=0.1522
\end{array}
$$

SCATTERING CCEFFICIENTS

\begin{tabular}{cccc}
$\theta$ & $\sigma / \sigma_{\mathrm{R}}$ & $\ell$ & \multicolumn{1}{c}{$s_{\ell}$} \\
$15^{\circ}$ & 0.999 & 1 & 0.3992 \\
30 & 0.962 & 2 & 1.145 \\
45 & 0.890 & 3 & 2.193 \\
60 & 0.790 & 4 & 3.517 \\
75 & 0.659 & 5 & 5.101 \\
90 & 0.537 & 6 & 5.929 \\
105 & 0.403 & 7 & 8.992 \\
120 & 0.277 & 8 & 11.28 \\
135 & 0.158 & 9 & 13.78 \\
150 & 0.084 & 10 & 15.49 \\
165 & 0.031 & 11 & 19.41 \\
180 & 0.013 & 12 & 22.52
\end{tabular}

STOPPING PONER

$$
(\mathrm{dE} / \mathrm{dr})_{\mathrm{E}_{\mathrm{O}}}=1.75 \mathrm{Mev}\left(\mathrm{cm}^{2} / \mathrm{g}\right)
$$

RESIDUAL RANGE

$$
\mathrm{r}_{\mathrm{O}}=5.83 \mathrm{~g} / \mathrm{cm}^{2}
$$

OTHER PARAMETERS

$$
\begin{array}{ll}
d_{1}=1.071 & A_{1}=0.2514 \\
\alpha=0.2370 & A_{2}=1.005 \\
A^{\text {PTI }}=0.4129 & A_{3}=-0.5297 \\
A^{\text {PLP }}=0.4788 & A_{4}=0.2734
\end{array}
$$

SCATTERING COEFFICIENTS

$\begin{array}{clll}\theta & \sigma / \sigma_{R} & l & s_{\ell} \\ 15^{\circ} & 0.999 & 1 & 0.2051 \\ 30 & 0.961 & 2 & 0.5920 \\ 45 & 0.888 & 3 & 1.140 \\ 60 & 0.788 & 4 & 1.838 \\ 75 & 0.666 & 5 & 2.679 \\ 90 & 0.532 & 6 & 3.655 \\ 105 & 0.397 & 7 & 4.762 \\ 120 & 0.269 & 8 & 5.995 \\ 135 & 0.159 & 9 & 7.351 \\ 150 & 0.074 & 10 & 8.827 \\ 165 & 0.021 & 11 & 10.42 \\ 180 & 0.002 & 12 & 12.12\end{array}$

\begin{tabular}{|c|c|c|}
\hline $\mathrm{J}(\mathrm{x})$ & $J(x)$ & $J(-x)$ \\
\hline 1.070 & 1.296 & 0.000 \\
\hline 1.087 & 1.325 & 0.000 \\
\hline 1.109 & 1.352 & 0.001 \\
\hline 1.137 & 1.373 & 0.001 \\
\hline 1.169 & 1.384 & 0.000 \\
\hline 1.204 & 1.378 & 0.000 \\
\hline 1.239 & 1.349 & 0.000 \\
\hline 1.273 & 1.297 & 0.000 \\
\hline 1.303 & 1.232 & 0.000 \\
\hline 1.326 & 1.157 & 0.000 \\
\hline 1.336 & 1.071 & 0.000 \\
\hline 1.324 & 0.969 & 0.000 \\
\hline 1.279 & 0.847 & 0.000 \\
\hline 1.184 & 0.698 & 0.000 \\
\hline 1.018 & 0.521 & 0.000 \\
\hline 0.767 & 0.324 & 0.000 \\
\hline 0.444 & 0.143 & 0.000 \\
\hline 0.143 & 0.033 & 0.000 \\
\hline 0.010 & 0.000 & 0.000 \\
\hline 0.000 & 0.000 & 0.000 \\
\hline
\end{tabular}

$\begin{array}{cc}\text { POINT } & \text { PLANE } \\ \text { ISOTROPIC } & \text { PERPENDICULAR } \\ \text { SOURCE } & \text { SOURCE }\end{array}$

$\begin{array}{cccc}x & J(x) & J(x) & J(-x) \\ 0.000 & 1.000 & 1.031 & 0.031 \\ 0.025 & 0.999 & 1.038 & 0.024 \\ 0.050 & 1.000 & 1.047 & 0.018 \\ 0.075 & 1.003 & 1.060 & 0.012 \\ & & & \\ 0.100 & 1.006 & 1.075 & 0.008 \\ 0.125 & 1.011 & 1.094 & 0.003 \\ 0.150 & 1.017 & 1.117 & 0.000 \\ 0.175 & 1.025 & 1.145 & -0.002 \\ 0.200 & 1.033 & 1.178 & -0.004 \\ 0.225 & 1.042 & 1.217 & -0.004 \\ 0.250 & 1.051 & 1.261 & -0.003 \\ 0.275 & 1.060 & 1.309 & -0.002 \\ 0.300 & 1.069 & 1.357 & -0.000 \\ 0.325 & 1.078 & 1.400 & 0.001 \\ 0.350 & 1.090 & 1.439 & 0.002 \\ 0.375 & 1.103 & 1.473 & 0.003 \\ 0.400 & 1.120 & 1.501 & 0.004 \\ 0.425 & 1.142 & 1.523 & 0.004 \\ 0.450 & 1.168 & 1.538 & 0.004 \\ 0.475 & 1.201 & 1.545 & 0.004\end{array}$

$$
z=6, E_{\sigma}=10.00 \mathrm{Mev}
$$

$\begin{array}{cc}\text { POINT } & \text { PLANE } \\ \text { ISOTROPIC } & \text { PERPENDICULAR } \\ \text { SOURCE } & \text { SCURCE }\end{array}$

$\begin{array}{cccc}x & J(x) & J(x) & J(-x) \\ 0.000 & 1.000 & 1.007 & 0.007 \\ 0.025 & 0.999 & 1.007 & 0.007 \\ 0.050 & 0.999 & 1.009 & 0.005 \\ 0.075 & 1.000 & 1.013 & 0.005 \\ & & & \\ 0.100 & 1.002 & 1.019 & 0.004 \\ 0.125 & 1.004 & 1.027 & 0.003 \\ 0.150 & 1.007 & 1.037 & 0.003 \\ 0.175 & 1.009 & 1.049 & 0.002 \\ 0.200 & 1.011 & 1.064 & 0.001 \\ 0.225 & 1.013 & 1.079 & -0.000 \\ 0.250 & 1.015 & 1.095 & -0.001 \\ 0.275 & 1.017 & 1.108 & -0.001 \\ 0.300 & 1.019 & 1.120 & -0.001 \\ 0.325 & 1.022 & 1.134 & -0.001 \\ 0.350 & 1.025 & 1.150 & 0.000 \\ 0.375 & 1.029 & 1.169 & 0.000 \\ 0.400 & 1.034 & 1.190 & 0.000 \\ 0.425 & 1.040 & 1.214 & 0.000 \\ 0.450 & 1.048 & 1.240 & 0.000 \\ 0.475 & 1.057 & 1.267 & 0.000\end{array}$

POINT PLANE

ISOTROPIC PERPENDICULAR SOURCE SOURCE

$\begin{array}{lrr}J(x) & J(x) & J(-x) \\ 1.239 & 1.543 & 0.003 \\ 1.281 & 1.531 & 0.002 \\ 1.324 & 1.509 & 0.000 \\ 1.367 & 1.474 & -0.001 \\ & & \\ 1.410 & 1.426 & -0.003 \\ 1.449 & 1.365 & -0.004 \\ 1.482 & 1.289 & -0.005 \\ 1.506 & 1.200 & -0.003 \\ & & \\ 1.514 & 1.097 & -0.001 \\ 1.501 & 0.981 & 0.001 \\ 1.457 & 0.853 & 0.003 \\ 1.372 & 0.714 & 0.003 \\ & & \\ 1.232 & 0.564 & 0.003 \\ 1.028 & 0.411 & 0.001 \\ 0.760 & 0.263 & -0.000 \\ 0.456 & 0.136 & -0.002 \\ & & \\ 0.184 & 0.048 & -0.002 \\ 0.033 & 0.008 & -0.001 \\ 0.001 & 0.000 & -0.000 \\ 0.000 & 0.000 & -0.000\end{array}$

POINT PLANE

ISOTROPIC PERPENDICULAR SOURCE SCURCE 
Table 1. Energy Dissipation Function, $J(x)$, and Input Data - Continued

ALTMINUS:

$Z=13, E_{0}=0.025 \mathrm{Mev}$

STOPPING POWER

$$
(\mathrm{dE} / \mathrm{dr})_{E_{0}}=8.50 \mathrm{Mev}\left(\mathrm{cm}^{2} / \mathrm{g}\right)
$$

RESIDUAL RANGGE

$$
\begin{aligned}
r_{0} & =0.00167 \mathrm{~g} / \mathrm{cm}^{2} \\
\text { OTHER PARAIETERS } & \\
a_{1}=2.049 & A_{1}=0.7544 \\
\alpha=-17.87 & A_{2}=0.4987 \\
{ }_{A}^{\text {PTI }}=1.500 & A_{3}=-0.3392 \\
{ }_{A}^{\text {PLP }}=1.729 & A_{4}=0.0861
\end{aligned}
$$

SCATTERING CCEEFICIENTS

$\begin{array}{cccc}\theta & \sigma / \sigma_{\mathrm{R}} & l & \mathrm{~s}_{\ell} \\ 15^{\circ} & 1.010 & 1 & 2.170 \\ 30 & 1.015 & 2 & 5.399 \\ 45 & 1.015 & 3 & 9.302 \\ 60 & 1.010 & 4 & 13.65 \\ 75 & 1.001 & 5 & 18.29 \\ 90 & 0.988 & 6 & 23.10 \\ 105 & 0.973 & 7 & 28.00 \\ 120 & 0.959 & 8 & 32.94 \\ 135 & 0.946 & 9 & 38.92 \\ 150 & 0.936 & 10 & 44.97 \\ 165 & 0.930 & 11 & 51.07 \\ 180 & 0.928 & 12 & 57.20\end{array}$

STOPPING PRIEF

$$
\left(d E_{i}^{\prime d r}\right)_{E_{O}}=3.14 \mathrm{Mev}\left(\mathrm{cm}^{2} / \mathrm{g}\right)
$$

RESIDIAL RANGE

$$
r_{0}=0.00561 \mathrm{~g} / \mathrm{cm}^{2}
$$

OTHER PARAMETERS

$$
\begin{array}{ll}
\mathrm{d}_{1}=2.190 & \mathrm{~A}_{1}=0.6818 \\
\alpha=33.52 & \mathrm{~A}_{2}=1.500 \\
{ }_{\mathrm{A}}^{\mathrm{PTI}}=0.1582 & \mathrm{~A}_{3}=-2.445 \\
{ }^{\mathrm{PLP}}=1.829 & \mathrm{~A}_{4}=1.260
\end{array}
$$

SCATTERING COEFFICIENTS

$\begin{array}{cccc}\theta & \sigma / \sigma & l & s \ell \\ 15^{\circ} & 1.013 & 1 & 2.126 \\ 30 & 1.017 & 2 & 5.440 \\ 45 & 1.013 & 3 & 9.587 \\ 60 & 0.999 & 4 & 14.36 \\ 75 & 0.979 & 5 & 19.59 \\ 90 & 0.955 & 6 & 25.18 \\ 105 & 0.928 & 7 & 31.04 \\ 120 & 0.902 & 8 & 37.10 \\ 135 & 0.879 & 9 & 43.29 \\ 150 & 0.861 & 10 & 49.56 \\ 165 & 0.850 & 11 & 55.89 \\ 180 & 0.846 & 12 & 62.24\end{array}$

$\begin{array}{cc}\text { POINT } & \text { PLANE } \\ \text { ISOTROPIC } & \text { PERPENDICULAR } \\ \text { SOURCE } & \text { SOURCE }\end{array}$

$\begin{array}{cccc}x & J(x) & J(x) & J(-x) \\ 0.000 & 1.000 & 2.009 & 1.009 \\ 0.025 & 1.026 & 2.224 & 0.809 \\ 0.050 & 1.080 & 2.419 & 0.640 \\ 0.075 & 1.161 & 2.593 & 0.506 \\ & & & \\ 0.100 & 1.265 & 2.743 & 0.410 \\ 0.125 & 1.391 & 2.870 & 0.350 \\ 0.150 & 1.531 & 2.973 & 0.316 \\ 0.175 & 1.679 & 3.054 & 0.292 \\ 0.200 & 1.832 & 3.111 & 0.268 \\ 0.225 & 1.990 & 3.145 & 0.241 \\ 0.250 & 2.154 & 3.153 & 0.213 \\ 0.275 & 2.325 & 3.135 & 0.183 \\ 0.300 & 2.499 & 3.090 & 0.153 \\ 0.325 & 2.674 & 3.018 & 0.122 \\ 0.350 & 2.844 & 2.920 & 0.092 \\ 0.375 & 3.006 & 2.797 & 0.064 \\ 0.400 & 3.153 & 2.653 & 0.041 \\ 0.425 & 3.280 & 2.492 & 0.024 \\ 0.450 & 3.378 & 2.316 & 0.013 \\ 0.475 & 3.440 & 2.131 & 0.008\end{array}$

$$
\mathrm{Z}=13, \mathrm{E}_{\mathrm{o}}=0.05 \mathrm{Mev}
$$

$\begin{array}{cc}\text { FOINT } & \text { PLANE } \\ \text { ISOTRPPIC } & \text { PERPENDICULAR } \\ \text { SOURCE } & \text { SOURCE }\end{array}$

$$
\begin{array}{llll}
x & J(x) & J(x) & J(-x)
\end{array}
$$

0.000

1.000

$$
1.972
$$$$
0.972
$$

0.025

1.017

2.180

5.773

0.050

1. :

2. 2

0.607

0.075

1.131

2.546

0.478

0.100

]. 22

0.1 .25

0.150

c. 175

1. 338

2.703

1.470

2. 842

0.385

1.620

2.960

0.328

0.295

0.200

0.225

1.783

3.055

0.270

0.250

0.275

1.958

3.124

2. 141

3.167

0.245

0.218

2. 330

3.181

0.191

0.300

0.325

2. 520

3.168

0.163

0.350

0.375

2.708

3.125

2.890

3.053

0.134

0.106

3.060

2.826

0.079

0.400

0.425

0.450

0.475
3.213

3. 342

2.675

3.440

2. 505

3.497

2. 319
2.122
0.034

0.018

0.008

0.004
POINT ISCTROPIC PERPENDICULAR SOURCE SOURCE

$\begin{array}{clll}x & J(x) & J(x) & J(-x) \\ 0.500 & 3.456 & 1.939 & 0.007 \\ 0.525 & 3.420 & 1.743 & 0.008 \\ 0.550 & 3.324 & 1.545 & 0.008 \\ 0.575 & 3.165 & 1.347 & 0.008\end{array}$

0.600

2.943

1.151

0.007

0.625

0.650

0.675

2.663

2.339

0.959

0.006

1.983

0.601

0.005

0.700

1.612

0.443

0.002

0.725

0.750

0.775

1. 241

0.887

0.573

0.305

0.000

$0.193-0.000$

0.800

0.825

0.320

$0.108-0.001$

0.850

0.875

0.143

0.046

0.008

$0.051-0.001$

$0.019-0.000$

$0.005-0.000$

$0.001-0.000$

0.900

0.925

0.001

0.000

$0.000-0.000$

0.950

0.975

0.000

$0.000-0.000$

$0.000-0.000$

0.000 
Table 1. Energy Dissipation Function, $J(x)$, and Input Data - Continued

\section{ALUMINUM}

$$
\mathrm{z}=13, \mathrm{E}_{\mathrm{o}}=0.10 \mathrm{Mev}
$$

STCPPING PONER

$$
(\mathrm{dE} / \mathrm{dr})_{E_{0}}=3.24 \mathrm{Mev}\left(\mathrm{cm}^{2} / \mathrm{g}\right)
$$

RESIDUAL RANGE

$$
r_{0}=0.0183 \mathrm{~g} / \mathrm{cm}^{2}
$$

OTHER PARAMETERS

$$
\begin{array}{ll}
d_{1}=2.145 & A_{1}=0.6884 \\
a=35.45 & A_{2}=0.9293 \\
{ }_{A} \text { PTI }=1.580 & A_{3}=-1.089 \\
{ }_{A} P L=1.833 & A_{4}=0.4714
\end{array}
$$

SCATTERING COEFFICIENTS

$\begin{array}{cccc}\theta & \sigma / \sigma_{\mathrm{R}} & \ell & \mathrm{s} \ell \\ 15^{\circ} & 1.015 & 1 & 2.086 \\ 30 & 1.017 & 2 & 5.478 \\ 45 & 1.004 & 3 & 9.840 \\ 60 & 0.997 & 4 & 14.97 \\ 75 & 0.941 & 5 & 20.74 \\ 90 & 0.898 & 6 & 27.02 \\ 105 & 0.852 & 7 & 33.73 \\ 120 & 0.808 & 8 & 40.80 \\ 135 & 0.769 & 9 & 48.18 \\ 150 & 0.738 & 10 & 55.79 \\ 165 & 0.719 & 11 & 63.62 \\ 180 & 0.712 & 12 & 71.62\end{array}$

STOPPING POWER

$$
(d E / d r)_{E_{O}}=2.22 \mathrm{Mov}\left(\mathrm{cm}^{2} / \mathrm{g}\right)
$$

RESIDUAL RAIGE

$$
\begin{array}{ll}
r_{0}=0.0570 \mathrm{~g} / \mathrm{cm}^{2} \\
\text { OTHER PARAMETERS } \\
d_{1}=2.118 & A_{1}=0.6285 \\
a=20.70 & A_{2}=0.9389 \\
A^{P T I}=1.575 & A_{3}=-1.056 \\
A^{P L P}=1.832 & A_{4}=0.4883
\end{array}
$$

SCATTERING COEFFICIENTS

$\begin{array}{cccc}\theta & \sigma / \sigma_{\mathrm{R}} & \ell & s_{\ell} \\ 15^{\circ} & 1.018 & 1 & 2.021 \\ 30 & 1.013 & 2 & 5.437 \\ 45 & 0.988 & 3 & 9.929 \\ 60 & 0.943 & 4 & 15.31 \\ 75 & 0.883 & 5 & 21.46 \\ 90 & 0.815 & 6 & 28.26 \\ 105 & 0.743 & 7 & 35.64 \\ 120 & 0.674 & 8 & 43.52 \\ 135 & 0.613 & 9 & 51.85 \\ 150 & 0.566 & 10 & 60.57 \\ 165 & 0.536 & 11 & 69.64 \\ 180 & 0.526 & 12 & 79.00\end{array}$

$\begin{array}{cc}\text { POINT } & \text { PLANE } \\ \text { ISOTROPIC } & \text { PERPENDICULAR } \\ \text { SOURCE } & \text { SOURCE }\end{array}$

$\begin{array}{cccc}\mathbf{x} & \mathrm{J}(\mathbf{x}) & \mathrm{J}(\mathbf{x}) & \mathrm{J}(\mathbf{- x}) \\ 0.000 & 1.000 & 1.916 & 0.916 \\ 0.025 & 1.021 & 2.129 & 0.718 \\ 0.050 & 1.066 & 2.324 & 0.555 \\ 0.075 & 1.133 & 2.501 & 0.429 \\ & & & \\ 0.100 & 1.221 & 2.659 & 0.342 \\ 0.125 & 1.327 & 2.797 & 0.292 \\ 0.150 & 1.451 & 2.913 & 0.267 \\ 0.175 & 1.589 & 3.004 & 0.248 \\ 0.200 & 1.739 & 3.068 & 0.228 \\ 0.225 & 1.900 & 3.105 & 0.204 \\ 0.250 & 2.069 & 3.112 & 0.179 \\ 0.275 & 2.243 & 3.091 & 0.151 \\ & & & \\ 0.300 & 2.420 & 3.040 & 0.122 \\ 0.325 & 2.594 & 2.961 & 0.093 \\ 0.350 & 2.764 & 2.856 & 0.066 \\ 0.375 & 2.924 & 2.726 & 0.042 \\ 0.400 & 3.068 & 2.577 & 0.024 \\ 0.425 & 3.192 & 2.412 & 0.012 \\ 0.450 & 3.287 & 2.235 & 0.006 \\ 0.475 & 3.346 & 2.049 & 0.004\end{array}$

$Z=13, E_{0}=0.20 \mathrm{Mev}$

$\begin{array}{cc}\text { POINT } & \text { PLANE } \\ \text { ISOTROPIC } & \text { PERPENDICULAR } \\ \text { SOURCE } & \text { SOURCE }\end{array}$

$x$

0.000

0.025

0.050

0.075

0.100

0.125

0.150

0.175

0.200

0.225

0.250

0.275

0.300

0.325

0.350

0.375

0.400

0.425

0.450

0.475

\section{$J(x)$}

1.000

1.016

1.053

1.110

1.185

1.276

1.383

1.503

1.635

1.778

1.928

2.085

2.245

2.405

2.563

2.714

$\mathrm{J}(\mathrm{x})$

1.822

2.022

2.205

2.372

2.521

2.653

2.763

2.850

2.910

2.943

2.948

2.924

$\mathrm{J}(-\mathrm{x})$

0.822

0.636

0.482

0.365

0.287

0.245

0.226

0.213

0.198

0.178

0.156

0.131

2.872

2.793

2.690

2.566

0.104

0.077

0.052

0.031

2.853

2.973

3.068

2.425

2.270

2.104

3.128
0.015

0.006

0.003

0.002
POINT

ISOTROPIC PERPENDICULAR SOURCE SOURCE

$\begin{array}{lll}J(x) & J(x) & J(-x) \\ 3.143 & 1.750 & 0.003 \\ 3.106 & 1.566 & 0.004 \\ 3.010 & 1.380 & 0.005 \\ 2.853 & 1.194 & 0.005 \\ & & \\ 2.640 & 1.009 & 0.005 \\ 2.376 & 0.829 & 0.004 \\ 2.072 & 0.658 & 0.003 \\ 1.737 & 0.499 & 0.002 \\ 1.386 & 0.358 & 0.001 \\ 1.038 & 0.238 & -0.000 \\ 0.714 & 0.143 & -0.000 \\ 0.438 & 0.075 & -0.000 \\ & & \\ 0.228 & 0.033 & -0.001 \\ 0.094 & 0.011 & -0.000 \\ 0.027 & 0.002 & -0.000 \\ 0.004 & 0.000 & -0.000 \\ 0.000 & 0.000 & -0.000 \\ 0.000 & 0.000 & -0.000 \\ 0.000 & 0.000 & -0.000 \\ 0.000 & 0.000 & -0.000\end{array}$

$\begin{array}{cc}\text { POINT } & \text { FLANE } \\ \text { ISOTROPIC } & \text { PERPENDICULAR }\end{array}$ SOURCE SOURCE

$\begin{array}{cccc}x & J(x) & J(x) & J(-x) \\ 0.500 & 3.361 & 1.857 & 0.004 \\ 0.525 & 3.321 & 1.660 & 0.006 \\ 0.550 & 3.220 & 1.462 & 0.006 \\ 0.575 & 3.052 & 1.263 & 0.006 \\ & & & \\ 0.600 & 2.819 & 1.067 & 0.006 \\ 0.625 & 2.529 & 0.877 & 0.005 \\ 0.650 & 2.194 & 0.696 & 0.003 \\ 0.675 & 1.831 & 0.528 & 0.002 \\ & & & \\ 0.700 & 1.456 & 0.379 & 0.001 \\ 0.725 & 1.090 & 0.252 & -0.000 \\ 0.750 & 0.750 & 0.152 & -0.001 \\ 0.775 & 0.462 & 0.081 & -0.001 \\ & & & \\ 0.800 & 0.243 & 0.035 & -0.001 \\ 0.825 & 0.102 & 0.012 & -0.000 \\ 0.850 & 0.030 & 0.003 & -0.000 \\ 0.875 & 0.005 & 0.000 & -0.000 \\ 0.900 & 0.000 & 0.000 & -0.000 \\ 0.925 & 0.000 & 0.000 & -0.000 \\ 0.950 & 0.000 & 0.000 & -0.000 \\ 0.975 & 0.000 & 0.000 & -0.000\end{array}$


STOPPING POWER

$$
(\mathrm{dE} / \mathrm{dr})_{\mathrm{E}_{\mathrm{O}}}=1.72 \mathrm{Mev}\left(\mathrm{cm}^{2} / \mathrm{g}\right)
$$

RESIDUAL RANGE

$$
r_{0}=0.162 \mathrm{~g} / \mathrm{cm}^{2}
$$

OTHER PARANETERS

$$
\begin{array}{ll}
d_{1}=2.094 & A_{1}=0.5291 \\
\alpha=9.679 & A_{2}=0.9542 \\
{ }_{A}^{\text {PTI }}=1.547 & A_{3}=-0.8196 \\
{ }_{A} \text { PLP }=1.805 & A_{4}=0.3363
\end{array}
$$

\section{SCATTERING COEFFICIENTS}

$\begin{array}{cccc}\theta & \sigma / \sigma_{\mathrm{R}} & \ell & s_{\ell} \\ 15^{\circ} & 1.019 & 1 & 1.898 \\ 30 & 1.008 & 2 & 5.218 \\ 45 & 0.968 & 3 & 9.666 \\ 60 & 0.903 & 4 & 15.08 \\ 75 & 0.818 & 5 & 21.33 \\ 90 & 0.722 & 6 & 28.34 \\ 105 & 0.621 & 7 & 36.03 \\ 120 & 0.525 & 8 & 44.34 \\ 135 & 0.441 & 9 . & 53.21 \\ 150 & 0.376 & 10 & 62.60 \\ 165 & 0.334 & 11 & 72.45 \\ 180 & 0.320 & 12 & 82.73\end{array}$

STOPPING POWER

$$
(d E / d r)_{E_{0}}=1.55 \mathrm{Mev}\left(\mathrm{cm}^{2} / \mathrm{g}\right)
$$

RESIDUAL RANGE

$$
r_{0}=0.349 \mathrm{~g} / \mathrm{cm}^{2}
$$

\section{OTHER PARAMETERS}

$$
\begin{array}{ll}
d_{1}=2.084 & A_{1}=0.452 \\
a^{P}=4.755 & A_{2}=0.9030 \\
A^{P T I}=1.497 & A_{3}=-0.5669 \\
A^{P L P}=1.747 & A_{4}=0.2118
\end{array}
$$

SCATTERING COEFFICIENTS

$\begin{array}{rlrc}\theta & \sigma / \sigma_{\mathrm{R}} & l & s_{l} \\ 15^{\circ} & 1.019 & 1 & 1.722 \\ 30 & 1.003 & 2 & 4.801 \\ 45 & 0.954 & 3 & 8.979 \\ 60 & 0.874 & 4 & 14.11 \\ 75 & 0.773 & 5 & 20.10 \\ 90 & 0.658 & 6 & 26.87 \\ 105 & 0.538 & 7 & 34.35 \\ 120 & 0.424 & 8 & 42.49 \\ 135 & 0.324 & 9 & 51.24 \\ 150 & 0.247 & 10 & 60.57 \\ 165 & 0.198 & 11 & 70.43 \\ 180 & 0.181 & 12 & 80.79\end{array}$

ALUMINUM

$Z=13, E_{0}=0.40 \mathrm{Mev}$

$\begin{array}{lc}\text { POTNT } & \text { PLANE } \\ \text { SOTROPIC } & \text { PERPENDICULAR } \\ \text { SOURCE } & \text { SOURCE }\end{array}$

$x$
0.000
0.025
0.050
0.075
0.100
0.125
0.150
0.175
0.200
0.225
0.250
0.275
0.300
0.325
0.350
0.375
0.400
0.425
0.450
0.475

$$
\mathrm{J}(\mathrm{x}) \quad \mathrm{J}(\mathrm{x}) \quad \mathrm{J}(-\mathrm{x})
$$

$$
1.000 \quad 1.680
$$

1.031

1.859

1.043

2.025

1.089

2.177

1.150

1.224

2.315

1.312

2.438

1.410

2.542

2.624

0.680

0.513

0.376

0.274

1.59

2.679

1.636

2.708

0.208

0.176

0.167

0.163

1.891

2.710

0.155
0.142

2.684

0.125

2.025

2.160

2.631

0.104

2.293

2.422

2.553

0.081

2.453

0.057

2.334

0.035

2.540

2.644

2.202

2.725

2.202
2.058
1.906
1.748

0.006

2.776

$-0.001$

0.000

$Z=13, E_{0}=0.70 \mathrm{Mev}$

$\begin{array}{cc}\text { POINT } & \text { PLANE } \\ \text { ISOTROPIC } & \text { PERPENDICULAR } \\ \text { SOURCE } & \text { SOURCE }\end{array}$

0.000

$\mathrm{J}(\mathrm{x})$

0.025

0.050

0.075

1.000

$\mathrm{J}(\mathrm{x})$

$\mathrm{J}(-\mathrm{x})$

1.009

1.535
1.686

1.031

1.828

1.066

1.961

0.535

0.394

0.278

0.100

0.125

1.113

1.170

2.085

0.193

0.150

0.175

1.238

2.199

0.139

1.314

2.299

0.115

2.378

0.113

0.200

0.225

1.400

2.434

1.492

2.465

0.115

0.250

0.275

1.591

1.696

2.471

0.113

0.107

0.095

0.300

0.325

1.804

1.914

2.450

0.080

0.350

0.375

2.024

2.404

0.062

2.131

.334

0.042

2.243

0.024

0.400

0.425

2.232

2.01

0.450

0.475
2.39

2.394

1.885

$\begin{array}{ll}1.748 & -0.003 \\ 1.605 & -0.002\end{array}$

$-0.003$

$-0.003$
0.500

0.525

0.550

0.575

0.600

0.625

0.650

0.675

0.700

0.725

0.750

0.775

0.800

0.825

0.850

0.875

0.900

0.925

0.950

0.975

0.000

0.000

0.000

1.561

0.942

0.652

0.401

0.210

.086
PLANE

ISOTROPIC PERPENDICULAR

SOURCE

$\begin{array}{ccc}J(x) & J(x) & J(-x) \\ 2.788 & 1.585 & 0.002 \\ 2.752 & 1.419 & 0.003 \\ 2.665 & 1.251 & 0.004 \\ 2.527 & 1.083 & 0.004\end{array}$

2.342

2.116

0.917

0.004

$0.755 \quad 0.003$

$0.600 \quad 0.002$

0.456

0.001

$\begin{array}{ll}0.328 & 0.001\end{array}$

$0.219-0.000$

$0.133-0.000$

$0.070-0.001$

$0.031-0.000$

$0.010-0.000$

$0.002-0.000$

$0.000-0.000$

$0.000-0.000$

$0.000-0.000$

$0.000-0.000$

$0.000-0.000$
POINT PLANE

ISOTROPIC PERPENDICULAR SOURCE

$\begin{array}{lll}J(x) & J(x) & J(-x) \\ 2.456 & 1.459 & 0.000 \\ 2.431 & 1.310 & 0.002 \\ 2.361 & 1.160 & 0.002 \\ 2.247 & 1.008 & 0.003 \\ & & \\ 2.093 & 0.858 & 0.003 \\ 1.906 & 0.711 & 0.003 \\ 1.678 & 0.569 & 0.002 \\ 1.426 & 0.437 & 0.001\end{array}$

$\begin{array}{llll}0.700 & 1.155 & 0.317 & 0.001\end{array}$

$\begin{array}{llll}0.725 & 0.880 & 0.214 & -0.000\end{array}$

$\begin{array}{llll}0.750 & 0.618 & 0.132 & -0.000\end{array}$

$\begin{array}{lllll}0.775 & 0.387 & 0.071 & -0.001\end{array}$

$\begin{array}{llll}0.800 & 0.207 & 0.032 & -0.000\end{array}$

$\begin{array}{llll}0.825 & 0.088 & 0.011 & -0.000\end{array}$

$\begin{array}{llll}0.850 & 0.026 & 0.002 & -0.000\end{array}$

0.875

0.004

$0.000-0.000$

0.900

0.925

0.000

$0.000-0.000$

0.950

0.000

$0.000-0.000$

0.975

0.000

$0.000-0.000$

$0.000-0.000$ 
Table 1. Energy Dissipation Function, $J(x)$, and Input Data - Continued

ALUMINUM

$$
z=13, \mathrm{E}_{0}=1.00 \mathrm{Mev}
$$

STOPPING POWER

$$
(d E / d r)_{E_{0}}=1.51 \operatorname{Mev}\left(\mathrm{cm}^{2} / \mathrm{g}\right)
$$

RESIDUAL RANGE

$$
r_{0}=0.545 \mathrm{~g} / \mathrm{cm}^{2}
$$

\section{OTHER PARAMETIRS}

$$
\begin{array}{ll}
d_{1}=2.093 & A_{1}=0.4150 \\
A_{1}=2.965 & A_{2}=0.8046 \\
A^{\text {PTI }}=1.447 & A_{3}=-0.2985 \\
A^{\text {PLP }}=1.690 & A_{4}=0.0790
\end{array}
$$

\section{SCATTERDYG COEFFICIENTS}

$\begin{array}{cccc}\theta & \sigma / \sigma_{R} & l & s l \\ 15^{\circ} & 1.020 & 1 & 1.565 \\ 30 & 1.001 & 2 & 4.395 \\ 45 & 0.947 & 3 & 8.262 \\ 60 & 0.861 & 4 & 13.04 \\ 75 & 0.752 & 5 & 18.64 \\ 90 & 0.628 & 6 & 25.01 \\ 105 & 0.499 & 7 & 32.07 \\ 120 & 0.377 & 8 & 39.78 \\ 135 & 0.270 & 9 & 48.11 \\ 150 & 0.187 & 10 & 57.02 \\ 165 & 0.134 & 11 & 66.47 \\ 180 & 0.116 & 12 & 76.44\end{array}$

STOPPING POWER

$$
(d E / d r)_{E_{0}}=1.51 \mathrm{Mev}\left(\mathrm{cm}^{2} / g\right)
$$

RESIDUAL RANGS

$$
r_{0}=1.21 \mathrm{~g} / \mathrm{cm}^{2}
$$

\section{OTHER PARAMBTERS}

$$
\begin{array}{ll}
d_{1}=2.112 & A_{1}=0.3274 \\
a_{1}=1.288 & A_{2}=0.9604 \\
A^{P T I}=1.294 & A_{3}=-0.4639 \\
A^{\text {PIP }}=1.513 & A_{4}=0.1761
\end{array}
$$

SCATTERING COEFFICIENTS

$\begin{array}{cccc}\theta & \sigma / \sigma_{R} & l & s q \\ 15^{\circ} & 1.020 & 1 & 1.189 \\ 30 & 0.999 & 2 & 3.373 \\ 45 & 0.939 & 3 & 6.392 \\ 60 & 0.846 & 4 & 10.16 \\ 75 & 0.727 & 5 & 14.62 \\ 90 & 0.594 & 6 & 19.71 \\ 105 & 0.455 & 7 & 25.41 \\ 120 & 0.323 & 8 & 31.69 \\ 135 & 0.207 & 9 & 38.50 \\ 150 & 0.118 & 10 & 45.83 \\ 165 & 0.061 & 11 & 53.65 \\ 180 & 0.042 & 12 & 61.95\end{array}$

$\begin{array}{cc}\text { POINT } & \text { PLANE } \\ \text { ISOTROPIC } & \text { PERPENDICULAR } \\ \text { SOURCE } & \text { SOURCE }\end{array}$

$\begin{array}{ccccc}x & J(x) & J(x) & J(-x) & x \\ 0.000 & 1.000 & 1.436 & 0.436 & 0.500 \\ 0.025 & 1.007 & 1.565 & 0.315 & 0.525 \\ 0.050 & 1.024 & 1.688 & 0.216 & 0.550 \\ 0.075 & 1.052 & 1.807 & 0.143 & 0.575 \\ 0.100 & 1.090 & 1.921 & 0.097 & 0.600 \\ 0.125 & 1.136 & 2.029 & 0.078 & 0.625 \\ 0.150 & 1.191 & 2.128 & 0.079 & 0.650 \\ 0.175 & 1.254 & 2.210 & 0.084 & 0.675 \\ & & & & \\ 0.200 & 1.324 & 2.269 & 0.086 & 0.700 \\ 0.225 & 1.401 & 2.306 & 0.083 & 0.725 \\ 0.250 & 1.483 & 2.318 & 0.076 & 0.750 \\ 0.275 & 1.570 & 2.305 & 0.065 & 0.775 \\ & & & & \\ 0.300 & 1.662 & 2.268 & 0.050 & 0.800 \\ 0.325 & 1.756 & 2.208 & 0.034 & 0.825 \\ 0.350 & 1.850 & 2.127 & 0.019 & 0.850 \\ 0.375 & 1.944 & 2.029 & 0.006 & 0.875 \\ 0.400 & 2.034 & 1.918 & -0.002 & 0.900 \\ 0.425 & 2.115 & 1.797 & -0.005 & 0.925 \\ 0.450 & 2.184 & 1.670 & -0.005 & 0.950 \\ 0.475 & 2.232 & 1.538 & -0.003 & 0.975\end{array}$

$z=13, E_{0}=2.00 \mathrm{Mov}$

$\begin{array}{cc}\text { POINT } & \text { PLANE } \\ \text { ISOTROPIC } & \text { PERPENDICULAR } \\ \text { SOURCE } & \text { SOURCE }\end{array}$

$\begin{array}{cccc}x & J(x) & J(x) & J(-x) \\ 0.000 & 1.000 & 1.252 & 0.252 \\ 0.025 & 1.002 & 1.333 & 0.177 \\ 0.050 & 1.012 & 1.415 & 0.114 \\ 0.075 & 1.027 & 1.500 & 0.065 \\ 0.100 & 1.049 & 1.589 & 0.034 \\ 0.125 & 1.076 & 1.683 & 0.021 \\ 0.150 & 1.109 & 1.778 & 0.022 \\ 0.175 & 1.148 & 1.865 & 0.030 \\ 0.200 & 1.192 & 1.938 & 0.036 \\ 0.225 & 1.241 & 1.993 & 0.040 \\ 0.250 & 1.294 & 2.030 & 0.040 \\ 0.275 & 1.353 & 2.048 & 0.038 \\ 0.300 & 1.416 & 2.045 & 0.033 \\ 0.325 & 1.482 & 2.021 & 0.026 \\ 0.350 & 1.552 & 1.976 & 0.017 \\ 0.375 & 1.624 & 1.911 & 0.007 \\ 0.400 & 1.698 & 1.828 & -0.001 \\ 0.425 & 1.770 & 1.731 & -0.006 \\ 0.450 & 1.837 & 1.623 & -0.008 \\ 0.475 & 1.895 & 1.508 & -0.007\end{array}$

$\begin{array}{cc}\text { POINT } & \text { PLANE } \\ \text { ISOTROPIC } & \text { PERPENDICULAR } \\ \text { SOURCE } & \text { SOURCE }\end{array}$

$\mathrm{J}(\mathrm{x}) \mathrm{J}(\mathrm{x}) \quad \mathrm{J}(-\mathrm{x})$

$2.253 \quad 1.402 \quad-0.001$

$2.238 \quad 1.264 \quad 0.001$

$\begin{array}{lll}2.184 & 1.124 & 0.002\end{array}$

$\begin{array}{lll}2.088 & 0.982 & 0.002\end{array}$

$\begin{array}{lll}1.956 & 0.841 & 0.003\end{array}$

$\begin{array}{lll}1.789 & 0.702 & 0.002\end{array}$

$\begin{array}{lll}1.589 & 0.567 & 0.002\end{array}$

$\begin{array}{lll}1.362 & 0.439 & 0.001\end{array}$

$$
\begin{array}{lll}
1.116 & 0.323 & 0.001
\end{array}
$$

$\begin{array}{lll}0.861 & 0.221 & 0.000\end{array}$

$\begin{array}{lll}0.614 & 0.138 & -0.000\end{array}$

$\begin{array}{llll}0.393 & 0.076 & -0.001\end{array}$

$\begin{array}{lll}0.216 & 0.035 & -0.000\end{array}$

$\begin{array}{lll}0.094 & 0.012=0.000\end{array}$

$0.029 \quad 0.003 \quad=0.000$

$\begin{array}{lll}0.005 & 0.000 & -0.000\end{array}$

$\begin{array}{lll}0.000 & 0.000 & -0.000 \\ 0.000 & 0.000 & -0.000 \\ 0.000 & 0.000 & -0.000 \\ 0.000 & 0.000 & -0.000\end{array}$

\section{POINT PLANE \\ ISOTROPIC PERPENDICULAR SOURCE SOURCE}

$\begin{array}{cccc}x & J(x) & J(x) & J(-x) \\ 0.500 & 1.936 & 1.388 & -0.005 \\ 0.525 & 1.953 & 1.265 & -0.002 \\ 0.550 & 1.936 & 1.139 & 0.000 \\ 0.575 & 1.882 & 1.010 & 0.001 \\ 0.600 & 1.788 & 0.879 & 0.002 \\ 0.625 & 1.659 & 0.748 & 0.003 \\ 0.650 & 1.499 & 0.619 & 0.002 \\ 0.675 & 1.313 & 0.493 & 0.002 \\ & & & \\ 0.700 & 1.105 & 0.375 & 0.001 \\ 0.725 & 0.883 & 0.267 & 0.000 \\ 0.750 & 0.658 & 0.175 & -0.000 \\ 0.775 & 0.446 & 0.102 & -0.001 \\ 0.800 & 0.263 & 0.050 & -0.001 \\ 0.825 & 0.127 & 0.019 & -0.000 \\ 0.850 & 0.045 & 0.005 & -0.000 \\ 0.875 & 0.009 & 0.001 & -0.000 \\ 0.900 & 0.001 & 0.000 & -0.000 \\ 0.925 & 0.000 & 0.000 & -0.000 \\ 0.950 & 0.000 & 0.000 & -0.000 \\ 0.975 & 0.000 & 0.000 & -0.000\end{array}$


Table 1. Energy Dissipation Function, $J(x)$, and Input Data - Continued

ALUMINUM

$Z=13, E_{0}=4.00 \mathrm{Mev}$

STOPPING POWER

$$
(\mathrm{dE} / \mathrm{dr})_{E_{\mathrm{O}}}=1.56 \mathrm{Mev}\left(\mathrm{cm}^{2} / \mathrm{g}\right)
$$

RESIDUAL RANGE

$$
r_{0}=2.51 \mathrm{~g} / \mathrm{cm}^{2}
$$

OTHER PARAMETERS

$$
\begin{array}{ll}
d_{1}=2.176 & A_{1}=0.2857 \\
\alpha^{P T I}=0.5808 & A_{2}=0.9244 \\
{ }^{P T I}=1.065 & A_{3}=-0.3746 \\
{ }^{P L P}=1.244 & A_{4}=0.1645
\end{array}
$$

SCATTERING COEFFICIENTS

$\begin{array}{cccc}\theta & \sigma \sigma_{R} & \ell & s_{\ell} \\ 15^{\circ} & 1.020 & 1 & 0.7995 \\ 30 & 0.998 & 2 & 2.284 \\ 45 & 0.936 & 3 & 4.355 \\ 60 & 0.840 & 4 & 6.961 \\ 75 & 0.718 & 5 & 10.07 \\ 90 & 0.580 & 6 & 13.64 \\ 105 & 0.438 & 7 & 17.66 \\ 120 & 0.302 & 8 & 22.11 \\ 135 & 0.183 & 9 & 26.96 \\ 150 & 0.091 & 10 & 32.21 \\ 165 & 0.033 & 11 & 37.84 \\ 180 & 0.013 & 12 & 43.84\end{array}$

STOPPING POWER

$$
(d E / d r)_{E_{O}}=1.66 \mathrm{Mev}\left(\mathrm{cm}^{2} / g\right)
$$

RESIDUAL RANGE

$$
r_{0}=6.22 \mathrm{~g} / \mathrm{cm}^{2}
$$

\section{OTHER PARAMETERS}

$$
\begin{array}{ll}
\mathrm{d}_{1}=2.288 & A_{1}=0.2366 \\
{ }=0.2169 & A_{2}=1.032 \\
A^{P T I}=0.7789 & A_{3}=-0.6144 \\
A^{P L P}=0.9059 & A_{4}=0.3458
\end{array}
$$

SCATTERING COEFFICIENTS

\begin{tabular}{cccc}
$\theta$ & $\sigma \sigma_{\mathrm{R}}$ & $l$ & \multicolumn{1}{c}{$s \ell$} \\
$15^{\circ}$ & 1.020 & 1 & 0.4078 \\
30 & 0.997 & 2 & 1.173 \\
45 & 0.935 & 3 & 2.251 \\
60 & 0.838 & 4 & 3.619 \\
75 & 0.714 & 5 & 5.261 \\
90 & 0.575 & 6 & 7.164 \\
105 & 0.431 & 7 & 9.317 \\
120 & 0.294 & 8 & 11.71 \\
135 & 0.174 & 9 & 14.34 \\
150 & 0.081 & 10 & 17.20 \\
165 & 0.023 & 11 & 20.27 \\
180 & 0.002 & 12 & 23.57
\end{tabular}

$\begin{array}{cc}\text { POINT } & \text { PLANE } \\ \text { ISOTROPIC } & \text { PERPENDICULAR } \\ \text { SOURCE } & \text { SOURCE }\end{array}$

$\begin{array}{cccc}\mathrm{x} & \mathrm{J}(\mathrm{x}) & \mathrm{J}(\mathrm{x}) & \mathrm{J}(\boldsymbol{- x}) \\ 0.000 & 1.000 & 1.107 & 0.107 \\ 0.025 & 1.000 & 1.119 & 0.098 \\ 0.050 & 1.004 & 1.140 & 0.088 \\ 0.075 & 1.010 & 0.075 & 0.077 \\ & & & \\ 0.100 & 1.020 & 1.228 & 0.064 \\ 0.125 & 1.033 & 1.303 & 0.049 \\ 0.150 & 1.049 & 1.400 & 0.032 \\ 0.175 & 1.069 & 1.507 & 0.015 \\ 0.200 & 1.091 & 1.604 & 0.000 \\ 0.225 & 1.118 & 1.686 & -0.010 \\ 0.250 & 1.148 & 1.752 & -0.017 \\ 0.275 & 1.181 & 1.800 & -0.020 \\ & & & \\ 0.300 & 1.219 & 1.829 & -0.018 \\ 0.325 & -1.262 & 1.837 & -0.012 \\ 0.350 & 1.308 & 1.825 & -0.004 \\ 0.375 & 1.359 & 1.793 & 0.005 \\ 0.400 & 1.414 & 1.744 & 0.013 \\ 0.425 & 1.472 & 1.683 & 0.016 \\ 0.450 & 1.530 & 1.612 & 0.015 \\ 0.475 & 1.584 & 1.533 & 0.011\end{array}$

$$
Z=13, E_{0}=10.00 \mathrm{Mev}
$$

$\begin{array}{cc}\text { POINT } & \text { PLANE } \\ \text { ISOTROPIC } & \text { PERPENDICULAR } \\ \text { SOURCE } & \text { SOURCE }\end{array}$

$\begin{array}{cccc}x & J(x) & J(x) & J(-x) \\ 0.000 & 1.000 & 1.023 & 0.023 \\ 0.025 & 0.999 & 1.027 & 0.020 \\ 0.050 & 1.001 & 1.034 & 0.016 \\ 0.075 & 1.003 & 1.044 & 0.013 \\ 0.100 & 1.008 & 1.061 & 0.010 \\ 0.125 & 1.014 & 1.085 & 0.008 \\ 0.150 & 1.021 & 1.120 & 0.006 \\ 0.175 & 1.028 & 1.164 & 0.004\end{array}$

0.200

0.225

1.037

1.211

0.003

0.250

\subsection{4}

1.051

1.256

0.002

0.275

1.057

1.342

0.002

0.300

1.062

0.325

0.350

$$
1.071
$$

$1.382 \quad 0.000$

1.083

$1.418-0.000$

$1.451-0.001$

0.375

1.099

$1.480-0.001$

$\begin{array}{llll}0.400 & 1.122 & 1.503 & -0.002 \\ 0.425 & 1.153 & 1.519 & -0.002 \\ 0.450 & 1.191 & 1.528 & -0.002 \\ 0.475 & 1.238 & 1.528 & -0.001\end{array}$

POINT ISOTROPIC SOURCE

PLANE

PERPENDICULAR SOURCE

$$
\mathrm{J}(\mathrm{x}) \mathrm{J}(\mathrm{x}) \quad \mathrm{J}(-\mathrm{x})
$$

$\begin{array}{lll}1.631 & 1.447 & 0.007\end{array}$

$\begin{array}{lll}1.666 & 1.366 & 0.003\end{array}$

$\begin{array}{lll}1.686 & 1.257 & 0.000\end{array}$

$\begin{array}{lll}1.687 & 1.151 & -0.002\end{array}$

$\begin{array}{lll}1.664 & 1.037 & -0.003\end{array}$

$\begin{array}{llll}1.613 & 0.917 & -0.004\end{array}$

$\begin{array}{llll}1.529 & 0.790 & -0.004\end{array}$

$\begin{array}{lll}1.409 & 0.659 & -0.003\end{array}$

$\begin{array}{llll}0.700 & 1.251 & 0.526 & -0.002\end{array}$

$\begin{array}{lllll}0.725 & 1.059 & 0.397 & -0.001\end{array}$

$\begin{array}{llll}0.750 & 0.839 & 0.277 & 0.001\end{array}$

$\begin{array}{llll}0.750 & 0.607 & 0.174 & 0.001\end{array}$

$\begin{array}{llll}0.800 & 0.386 & 0.093 & 0.001\end{array}$

$\begin{array}{llll}.825 & 0.203 & 0.040 & 0.001\end{array}$

$\begin{array}{llll}0.900 & 0.002 & 0.000 & 0.000\end{array}$

$\begin{array}{llll}0.925 & 0.000 & 0.000 & 0.000\end{array}$

$\begin{array}{llll}0.950 & 0.000 & 0.000 & 0.000\end{array}$

$\begin{array}{lll}0.000 & 0.000 & 0.000 \\ 0.000 & 0.000 & 0.000\end{array}$

$x$
0.500
0.525
0.550
0.575

0.600

0.625

0.650

0.675

0.700

0.725

0.750

0.775

0.800

POINT PLANE ISOTROPIC PERPENDICULAR SOURCE SOURCE

$\mathrm{J}(\mathrm{x}) \quad \mathrm{J}(\mathrm{x}) \quad \mathrm{J}(-\mathrm{x})$

1.290

1.517

1.341

1.492

1.390

1.453

1.432

1.397

$-0.001$

0.000

0.001

0.002

$1.465 \quad 1.324$

1.485

1.486

1.232

0.003

0.003

1.462

1.122

0.003

1.408

1. 316

1.181

0.999

0.001

0.825

0.850

0.875

0.778

0.865

$-0.001$

0.724

0.580

$-0.003$

$-0.003$

0.999

0.437

$-0.002$

0.900

0.925

0.950

0.975
0.533

0.298

0.300

0.179

0.086

0.1190 .028

$-0.001$

0.001

0.001

0.001

$0.027 \quad 0.005$

$0.002 \quad 0.000$

0.000

$0.000 \quad 0.000$

$0.000 \quad 0.000$

0.000

0.000

0.000 
Table 1. Energy Dissipation Function, $J(x)$, and Input Data - Continued

COPPER

STOPPING POWER

$$
(\mathrm{dE} / \mathrm{dr})_{E_{0}}=6.91 \mathrm{Mev}\left(\mathrm{cm}^{2} / \mathrm{g}\right)
$$

RESIDUAL RANGE

$$
r_{0}=0.00210 \mathrm{~g} / \mathrm{cm}^{2}
$$

OTHER PARAMETERS

$$
\begin{array}{ll}
d_{1}=3.826 & A_{1}=0.6465 \\
a=-4.745 & A_{2}=0.8729 \\
A^{P T I}=3.023 & A_{3}=-0.9436 \\
{ }_{A} P L=3.431 & A_{4}=0.4242
\end{array}
$$

SCATTERING COEFFICIENTS

$\begin{array}{rlll}\theta & \sigma / \sigma & l & s_{l} \\ 15^{\circ} & 1.013 & 1 & 4.847 \\ 30 & 1.035 & 2 & 11.32 \\ 45 & 1.055 & 3 & 18.48 \\ 60 & 1.066 & 4 & 25.84 \\ 75 & 1.070 & 5 & 33.12 \\ 90 & 1.066 & 6 & 40.14 \\ 105 & 1.059 & 7 & 46.80 \\ 120 & 1.050 & 8 & 53.03 \\ 135 & 1.041 & 9 & 59.51 \\ 150 & 1.034 & 10 & 65.07 \\ 165 & 1.030 & 11 & 71.36 \\ 180 & 1.028 & 12 & 76.81\end{array}$

STOPPING POWER

$$
(\mathrm{dE} / \mathrm{dr})_{\mathrm{E}_{\mathrm{O}}}=4.26 \mathrm{Mev}\left(\mathrm{cm}^{2} / \mathrm{g}\right)
$$

RESIDUAL RANGE

$$
r_{0}=0.00689 \mathrm{~g} / \mathrm{cm}^{2}
$$

OTHER PARAIETERS

$$
\begin{array}{ll}
d_{1}=4.779 & A_{1}=0.5326 \\
\alpha=-22.11 & A_{2}=1.589 \\
A^{P T I}=3.628 & A_{3}=-1.011 \\
{ }_{A}{ }^{P L P}=4.130 & A_{4}=-0.1106
\end{array}
$$

\section{SCATTERING COEFFICIENTS}

$\begin{array}{clcc}\theta & \sigma / \sigma_{R} & l & s_{l} \\ 15^{\circ} & 1.026 & 1 & 5.006 \\ 30 & 1.054 & 2 & 12.32 \\ 45 & 1.072 & 3 & 21.37 \\ 60 & 1.076 & 4 & 31.28 \\ 75 & 1.067 & 5 & 41.80 \\ 90 & 1.047 & 6 & 52.64 \\ 105 & 1.022 & 7 & 63.61 \\ 120 & 0.995 & 8 & 74.57 \\ 135 & 0.970 & 9 & 85.41 \\ 50 & 0.950 & 10 & 96.03 \\ 65 & 0.937 & 11 & 106.4 \\ 180 & 0.932 & 12 & 116.5\end{array}$

$$
Z=29, E_{0}=0.025 \mathrm{Mev}
$$

POINT ISOTROPIC PERPENDICULAR SOURCE SOURCE

$x$
0.000
0.025
0.050
0.075
0.100
0.125
0.150
0.175
0.200
0.225
0.250
0.275
0.300
0.325
0.350
0.375
0.400
0.425
0.450
0.475

$\mathrm{J}(\mathrm{x})$ $\mathrm{J}(\mathrm{x}) \quad \mathrm{J}(-\mathrm{x})$

1.000

1.069

1.250

1.519

3.115

3.430

3.678

3.850

2.115

1.792

1.504

1.254

\subsection{9}

2.211

3.942

2.575

3.951

1.042

2.916

3.883

3.749

0.869

0.620

3.218

3.566

0.530

3.478

3.354

3.697

3.124

0.450

0.375

3.823

3.997

2.631

4.060

2.374

4.056

2.114

3.979

1.856

0.238

0.179

0.129

\subsection{8}

1.605

3.603

1.605
1.365
1.139
0.930

3.313
2.968

0.057
0.036
0.022
0.015

$Z=29, E_{0}=0.05 \mathrm{Mev}$

$\begin{array}{cc}\text { POINT } & \text { PLANE } \\ \text { ISCTROPIC } & \text { PERPENDICULAR } \\ \text { SOURCE } & \text { SOURCE }\end{array}$

SOURCE

SOURCE

$x$
0.000
0.025
0.050
0.075
0.100
0.125
0.150
0.175

$\mathrm{J}(\mathrm{x})$

$$
\mathrm{J}(\mathrm{x})
$$

1.000

1.108

3.230

3.657

1.344

1.676

3.971

4.171

2.067

2.483

4.262

1.190

2.895

4.249

0.974

3.278

4.144

3.972

0.200

0.225

0.250

3.617

3.743

3.901

4.123

3.476

3.183

0.502

0.275

4.271

2.875

0.300

0.325

4.335

2.560

0.249

0.350

0.375

4.310

2.247

0.180

4.192

1.943

3.986

1.653

0.400

3.700

1.381

0.425

0.450

0.475
2.946

2.518

1.131

0.905

0.705 $x$
0.500
0.525
0.550
0.575

0.600

0.625

0.650

0.675

0.700

0.725

0.750

0.775

0.800

0.825

0.850

0.875

0.900

0.925

0.950

0.975
POINT

ISOTROPIC PERPENDICULAR SOURCE

SOURCE

$\begin{array}{ccc}J(x) & J(x) & J(-x) \\ 2.585 & 0.741 & 0.011 \\ 2.183 & 0.575 & 0.009 \\ 1.782 & 0.432 & 0.007 \\ 1.402 & 0.313 & 0.006 \\ & & \\ 1.058 & 0.217 & 0.004 \\ 0.760 & 0.142 & 0.002 \\ 0.514 & 0.087 & 0.001 \\ 0.323 & 0.049 & 0.000 \\ & & \\ 0.184 & 0.025 & -0.000 \\ 0.093 & 0.011 & -0.000 \\ 0.040 & 0.004 & -0.000 \\ 0.014 & 0.001 & -0.000 \\ & & \\ 0.003 & 0.000 & -0.000 \\ 0.001 & 0.000 & -0.000 \\ 0.000 & 0.000 & -0.000 \\ 0.000 & 0.000 & -0.000 \\ & & \\ 0.000 & 0.000 & -0.000 \\ 0.000 & 0.000 & -0.000 \\ 0.000 & 0.000 & -0.000 \\ 0.000 & 0.000 & -0.000\end{array}$

$\mathrm{J}(-\mathrm{x})$

2.230

1.815

1.469

0.811

0.590

0.415

0.329

0.124

0.081

0.052

POINT

ISOTROPIC PERPENDICULAR SOURCE

$x$

0.015

0.011
0.500

0.550

0.575

0.600

0.625

0.650

0.675

0.700

0.725

0.750

0.775

0.800

0.825

0.850

0.875

0.900

0.925

0.950

J(x)

1.668

1.283

0.944

0.658

0.430

0.259

0.141

0.067

0.027

0.009

0.002

0.975
0.000

0.000

0.000

0.000

0.000

0.000

0.000

0.000
PLANE SOURCE

$$
\mathrm{J}(\mathrm{x}) \quad \mathrm{J}(-\mathrm{x})
$$

0.534

0.391

0.274

0.184

0.009

0.007

$$
0.005
$$

0.116

0.069

0.037

0.018

0.001

0.000

$-0.000$

$0.008-0.000$

$0.003-0.000$

$0.001-0.000$

$0.000-0.000$

$0.000-0.000$

$0.000-0.000$

$0.000-0.000$

$0.000-0.000$

$0.000=0.000$

$0.000-0.000$

$0.000-0.000$

$0.000-0.000$ 
STOPPING POWER

$$
(\mathrm{dE} / \mathrm{d} r)_{E_{0}}=2.72 \mathrm{Mev}\left(\mathrm{om}^{2} / \mathrm{g}\right)
$$

RESIDUAL RANGE

$$
r_{0}=0.0221 \mathrm{~g} / \mathrm{cm}^{2}
$$

OTHER PARAMETERS

$$
\begin{array}{ll}
d_{1}=4.971 & A_{1}=0.6446 \\
a^{2}=-45.31 & A_{2}=1.054 \\
A^{\text {PTI }}=3.808 & A_{3}=-1.240 \\
A^{P L P}=4.342 & A_{4}=0.5412
\end{array}
$$

SCATTERING COETFICIENTS

$\begin{array}{cccc}\theta & \sigma / \sigma_{R} & l & s_{l} \\ 15^{\circ} & 1.038 & 1 & 5.083 \\ 30 & 1.070 & 2 & 13.01 \\ 45 & 1.082 & 3 & 22.86 \\ 60 & 1.073 & 4 & 34.12 \\ 75 & 1.045 & 5 & 46.44 \\ 90 & 1.003 & 6 & 59.56 \\ 105 & 0.952 & 7 & 73.27 \\ 120 & 0.900 & 8 & 87.40 \\ 135 & 0.852 & 9 & 101.8 \\ 150 & 0.813 & 10 & 116.4 \\ 165 & 0.789 & 11 & 131.1 \\ 180 & 0.780 & 12 & 145.8\end{array}$

STOPPING POWER

$$
(\mathrm{dE} / \mathrm{dr})_{\mathrm{E}_{0}}=1.89 \mathrm{Mev}\left(\mathrm{cm}^{2} / \mathrm{B}\right)
$$

RESIDUAL RANGE

$$
r_{0}=0.0678 \mathrm{~g} / \mathrm{cm}^{2}
$$

OTHER PARAMETERS

$$
\begin{array}{ll}
d_{1}=5.125 & A_{1}=0.6033 \\
a=39.73 & A_{2}=1.008 \\
A^{\text {PTI }}=3.901 & A_{3}=-1.143 \\
A^{\text {PLP }}=4.452 & A_{4}=0.5313
\end{array}
$$

SCATTERING COEFFICIENTS

$\begin{array}{cccc}\theta & \sigma / \sigma_{R} & l & s_{l} \\ 15^{\circ} & 1.050 & 1 & 4.999 \\ 30 & 1.082 & 2 & 13.19 \\ 45 & 1.085 & 3 & 23.66 \\ 60 & 1.057 & 4 & 35.94 \\ 75 & 1.004 & 5 & 49.69 \\ 90 & 0.931 & 6 & 64.66 \\ 105 & 0.848 & 7 & 80.66 \\ 120 & 0.763 & 8 & 91.52 \\ 135 & 0.687 & 9 & 115.08 \\ 150 & 0.626 & 10 & 133.24 \\ 165 & 0.587 & 11 & 151.89 \\ 180 & 0.573 & 12 & 170.98\end{array}$

COPPER

$$
Z=29, E_{0}=0.10 \mathrm{Mev}
$$

$$
\begin{array}{cc}
\text { POINT } & \text { PLANE } \\
\text { ISOTROPIC } & \text { PERPENDICULAR } \\
\text { SOURCE } & \text { SOURCE }
\end{array}
$$

$\begin{array}{llll}x & J(x) & J(x) & \mathrm{J}(-x)\end{array}$

0.000

0.025

0.050

0.075

0.100

0.125

0.150

0.175

0.200

0.225

0.250

0.275

0.300

0.325

0.350

0.375

0.400

0.425

0.450

0.475

\subsection{0}

1.079

1.279

1.572

3.211

3.596

3.870

4.037

2.211

1.812

1.479

1.210

1.926

4.101

0.998

2.310

2.700

4.071

3.963

3.074

3.792

0.834

0.599

\subsection{5}

3.575

3.712

3.952

4.123

3.326

3.056

0.502

0.410

0.323

4.214

2.771

0.245

2.480

4.218

2.189

0.178

4.132

3.957

1.903

0.124

1.627

0.083

3.698

1.366

3.368

1.122

2.983

2.561

0.899

0.034

0.021

0.013

0.010

$x$
0.500
0.525
0.550
0.575

0.600

0.625

0.650

0.675

0.700

0.725

0.750

0.775

0.800

0.825

0.850

0.875

0.900

0.925

0.950

0.975
POINT

ISOTROPIC PERPENDICULAR SOURCE SOURCE

$J(x)$

$\mathrm{J}(\mathrm{x}) \quad \mathrm{J}(-\mathrm{x})$

2.126

1.700

1.302

0.950

0.529

0.385

0.268

0.177

0.007

0.005

0.004

0.654

0.420

0.247

0.130

0.110

$0.034-0.000$

$\begin{array}{ll}0.034 & -0.000 \\ 0.016 & -0.000\end{array}$

$\begin{array}{llll}0.060 & 0.006 & -0.000\end{array}$

0.023

0.007

0.002

$0.002-0.000$

$0.001-0.000$

$0.000-0.000$

$\begin{array}{llll}0.000 & 0.000 & -0.000\end{array}$

$\begin{array}{llll}0.000 & 0.000 & -0.000\end{array}$

$\begin{array}{llll}0.000 & 0.000 & -0.000\end{array}$

0.000

$0.000-0.000$

$\begin{array}{llll}0.000 & 0.000 & -0.000\end{array}$

$\begin{array}{llll}0.000 & 0.000 & -0.000\end{array}$

$\begin{array}{llll}0.000 & 0.000 & -0.000\end{array}$

0.000

$0.000-0.000$ $z=29, E_{0}=0.20 \mathrm{Nov}$

$\begin{array}{cc}\text { POINT } & \text { PLANE } \\ \text { ISOTROPIC } & \text { PERPENDICULAR } \\ \text { SOURCE } & \text { SOURCE }\end{array}$

SOURCE SOURCE

$\begin{array}{clll}x & J(x) & J(x) & J(-x) \\ 0.000 & 1.000 & 3.081 & 2.081 \\ 0.025 & 1.070 & 3.470 & 1.674 \\ 0.050 & 1.253 & 3.737 & 1.345 \\ 0.075 & 1.520 & 3.886 & 1.090 \\ 0.100 & 1.845 & 3.928 & 0.898 \\ 0.125 & 2.201 & 3.880 & 0.755 \\ 0.150 & 2.564 & 3.760 & 0.643 \\ 0.175 & 2.913 & 3.591 & 0.547 \\ 0.200 & 3.234 & 3.384 & 0.456 \\ 0.225 & 3.513 & 3.150 & 0.371 \\ 0.250 & 3.737 & 2.895 & 0.291 \\ 0.275 & 3.895 & 2.624 & 0.219 \\ 0.300 & 3.977 & 2.344 & 0.158 \\ 0.325 & 3.976 & 2.063 & 0.107 \\ 0.350 & 3.889 & 1.787 & 0.069 \\ 0.375 & 3.718 & 1.521 & 0.041 \\ 0.400 & 3.469 & 1.271 & 0.024 \\ 0.425 & 3.153 & 1.040 & 0.014 \\ 0.450 & 2.785 & 0.830 & 0.010 \\ 0.475 & 2.385 & 0.645 & 0.007\end{array}$

POINT

PLANE

ISOTROPIC PERPENDICULAR SOURCE

SOURCE

$J(x)$

$x$

0.500

0.525

0.550

0.575

0.600

0.625

0.650

0.675

0.700

0.725

0.750

0.775

$J(x)$

$J(x)$

$J(-x)$

1.974
1.571

0.485

1.196

0.351

0.865

0.243

0.006

0.589

0.372

0.215

0.111

0.159

0.002

$\begin{array}{lll}0.098 & 0.001\end{array}$

0.055

0.028

.000

0.050

0.018

0.005

0.001

0.00

0.002

0.000

0.800

0.825

0.850

0.000

0.000

0.000

0.875

0.000

$0.000-0.000$

0.900

0.000

$0.000-0.000$

0.925

0.950

0.000

$0.000-0.000$

0.000

$0.000-0.000$

0.975

0.000

$\begin{array}{ll}0.000 & -0.000 \\ 0.000 & -0.000\end{array}$ 
CCPPER

STOPPING PCWER

$$
(d E / d r)_{\Sigma_{0}}=1.47 \mathrm{Yev}\left(\mathrm{cm}^{2} / g\right)
$$

RISIDUAL RANGE

$$
r_{0}=0.191 \mathrm{~g} / \mathrm{cm}^{2}
$$

OTHER PARA1ETERS

$$
\begin{array}{ll}
d_{1}=5.159 & A_{1}=0.5130 \\
d_{A I}=11.06 & A_{2}=1.003 \\
A^{P T I}=3.857 & A_{3}=-0.8755 \\
A^{P L P}=4.406 & A_{4}=0.3598
\end{array}
$$

SCATTERING COEFFICIENTS

$\begin{array}{cccc}\theta & \sigma / \sigma_{R} & l & s_{l} \\ 15^{\circ} & 1.059 & 1 & 4.732 \\ 30 & 1.089 & 2 & 12.80 \\ 45 & 1.080 & 3 & 23.38 \\ 60 & 1.032 & 4 & 36.03 \\ 75 & 0.952 & 5 & 50.45 \\ 90 & 0.847 & 6 & 66.42 \\ 105 & 0.729 & 7 & 83.74 \\ 120 & 0.611 & 8 & 102.3 \\ 135 & 0.505 & 9 & 121.9 \\ 150 & 0.420 & 10 & 142.4 \\ 165 & 0.366 & 11 & 163.8 \\ 180 & 0.348 & 12 & 185.9\end{array}$

STOPPING PONER

$$
(d E / d r)_{E_{0}}=1.33 \mathrm{Vev}\left(\mathrm{cm}^{2} / \mathrm{g}\right)
$$

RESIDOAL RANGE

\begin{tabular}{|c|c|c|c|c|c|c|c|}
\hline \multirow[b]{2}{*}{$x$} & \multirow{2}{*}{$\begin{array}{l}\text { POINT } \\
\text { ISOTROPIC } \\
\text { SOURCE } \\
J(x)\end{array}$} & \multicolumn{2}{|c|}{$\begin{array}{l}\text { PLANE } \\
\text { PERPENDICULAR } \\
\text { SOURCE }\end{array}$} & \multirow{2}{*}{\multicolumn{2}{|c|}{$\begin{array}{l}\text { POINT } \\
\text { ISOTROPIC } \\
\text { SOURCE } \\
J(x)\end{array}$}} & \multicolumn{2}{|c|}{$\begin{array}{c}\text { PLANE } \\
\text { PERPENDICULAF } \\
\text { SOURCE }\end{array}$} \\
\hline & & $J(x)$ & $J(-x)$ & & & $J(x)$ & $J(-x)$ \\
\hline $\begin{array}{l}0.000 \\
0.025 \\
0.050 \\
0.075\end{array}$ & $\begin{array}{l}1.000 \\
1.059 \\
1.214 \\
1.442\end{array}$ & $\begin{array}{l}2.806 \\
3.161 \\
3.416 \\
3.571\end{array}$ & $\begin{array}{l}1.805 \\
1.441 \\
1.148 \\
0.921\end{array}$ & & & $\begin{array}{l}0.449 \\
0.324 \\
0.224 \\
0.147\end{array}$ & $\begin{array}{l}0.004 \\
0.003 \\
0.002 \\
0.002\end{array}$ \\
\hline & $\begin{array}{l}1.721 \\
2.027 \\
2.341 \\
2.644\end{array}$ & $\begin{array}{l}3.633 \\
3.608 \\
3.507 \\
3.345\end{array}$ & $\begin{array}{l}0.753 \\
0.631 \\
0.539 \\
0.459\end{array}$ & & & $\begin{array}{l}0.090 \\
0.051 \\
0.027 \\
0.012\end{array}$ & $\begin{array}{r}0.001 \\
0.000 \\
-0.000 \\
-0.000\end{array}$ \\
\hline $\begin{array}{l}0.200 \\
0.225 \\
0.250 \\
0.275\end{array}$ & $\begin{array}{l}2.924 \\
3.168 \\
3.364 \\
3.502\end{array}$ & $\begin{array}{l}3.138 \\
2.902 \\
2.652 \\
2.396\end{array}$ & $\begin{array}{l}0.382 \\
0.304 \\
0.232 \\
0.171\end{array}$ & & & $\begin{array}{l}0.005 \\
0.002 \\
0.000 \\
0.000\end{array}$ & $\begin{array}{l}-0.000 \\
-0.000 \\
-0.000 \\
-0.000\end{array}$ \\
\hline $\begin{array}{l}0.300 \\
0.325 \\
0.350 \\
0.375\end{array}$ & $\begin{array}{l}3.573 \\
3.570 \\
3.492 \\
3.339\end{array}$ & $\begin{array}{l}2.140 \\
1.886 \\
1.638 \\
1.400\end{array}$ & $\begin{array}{l}0.121 \\
0.083 \\
0.055 \\
0.035\end{array}$ & & & $\begin{array}{l}0.000 \\
0.000 \\
0.000 \\
0.000\end{array}$ & $\begin{array}{l}-0.00 \\
-0.00 \\
=0.00 \\
=0.00\end{array}$ \\
\hline $\begin{array}{l}0.425 \\
0.450 \\
0.475\end{array}$ & $\begin{array}{l}3.117 \\
2.837 \\
2.511 \\
2.156\end{array}$ & $\begin{array}{l}1.173 \\
0.962 \\
0.769 \\
0.597\end{array}$ & $\begin{array}{l}0.013 \\
0.008 \\
0.005\end{array}$ & $\begin{array}{l}0.950 \\
0.975\end{array}$ & $\begin{array}{l}0.000 \\
0.000 \\
0.000 \\
0.800\end{array}$ & $\begin{array}{l}0.000 \\
0.000 \\
0.000 \\
0.000\end{array}$ & $\begin{array}{l}-0.00 \\
-0.00 \\
-0.00 \\
-0.00\end{array}$ \\
\hline
\end{tabular}

$$
r_{0}=0.409 \mathrm{~g} / \mathrm{cm}^{2}
$$

OTHER PARAMETERS

$$
\begin{array}{ll}
d_{1}=5.172 & A_{1}=0.4427 \\
d_{A}=5.029 & A_{2}=0.0354 \\
A^{\mathrm{PTI}}=3.717 & A_{3}=-0.6005 \\
A^{\mathrm{PLP}}=4.249 & A_{4}=0.2214
\end{array}
$$

SCATTERING COETFICIENTS

$\begin{array}{cccc}\theta & \sigma / \sigma_{9} & l & s l \\ 15^{\circ} & 1.064 & 1 & 4.314 \\ 30 & 1.091 & 2 & 11.86 \\ 45 & 1.014 & 3 & 21.91 \\ 60 & 1.013 & 4 & 34.08 \\ 75 & 0.914 & 5 & 48.11 \\ 90 & 0.788 & 6 & 63.81 \\ 105 & 0.648 & 7 & 81.02 \\ 120 & 0.508 & 8 & 99.59 \\ 135 & 0.382 & 9 & 119.4 \\ 150 & 0.282 & 10 & 140.4 \\ 165 & 0.218 & 11 & 162.4 \\ 180 & 0.196 & 12 & 185.4\end{array}$

$$
z=29, E_{0}=0.40 \mathrm{Nev}
$$

$z=29, E_{0}=0.70 \mathrm{Mev}$

$\begin{array}{lc}\text { POINT } & \text { PLANE } \\ \text { ISOTROPIC } & \text { PERPENDICULAR } \\ \text { SOURCE } & \text { SOURCE }\end{array}$

$\begin{array}{lllll}x & J(x) & J(x) & J(-x) & x \\ 0.000 & 1.000 & 2.523 & 1.523 & 0.500 \\ 0.025 & 1.045 & 2.846 & 1.194 & 0.525 \\ 0.050 & 1.166 & 3.086 & 0.932 & 0.550\end{array}$

POINT

PLANE

ISOTROPIC PERPENDICULAR SOURCE SOURCE

$\mathrm{J}(\mathrm{x}) \quad \mathrm{J}(\mathrm{x}) \quad \mathrm{J}(-\mathrm{x})$

$\begin{array}{lll}1.679 & 0.442 & 0.003\end{array}$

$\begin{array}{lll}1.356 & 0.323 & 0.002\end{array}$

$1.048 \quad 0.226 \quad 0.002$

$\begin{array}{llllllll}0.075 & 1.348 & 3.242 & 0.735 & 0.575 & 0.771 & 0.150 & 0.001\end{array}$

$\begin{array}{llllllll}0.100 & 1.574 & 3.315 & 0.595 & 0.600 & 0.534 & 0.094 & 0.001\end{array}$

$\begin{array}{lllll}0.125 & 1.829 & 3.310 & 0.500 & 0.625\end{array}$

$\begin{array}{lllll}0.150 & 2.094 & 3.232 & 0.431 & 0.650\end{array}$

0.345

0.204

0.108

$0.054 \quad 0.000$

$0.029-0.000$

$\begin{array}{lllll}0.175 & 2.356 & 3.092 & 0.372 & 0.675\end{array}$

$\begin{array}{lllll}0.200 & 2.599 & 2.906 & 0.310 & 0.700 \\ 0.225 & 2.810 & 2.690 & 0.246 & 0.725\end{array}$

0.050

$0.013=0.000$

0.250

2.980

$2.462 \quad 0.185$

0.275

3.100

$2.231 \quad 0.135$

0.750

0.020

$0.005=0.000$

0.006

$0.002-0.000$

0.001

$0.000-0.000$

$0.000-0.000$

0.300
0.325

$\begin{array}{lll}3.165 & 1.999 & 0.094\end{array}$

0.800

0.325

$\begin{array}{lll}3.170 & 1.769 & 0.064\end{array}$

0.375

$\begin{array}{llll}3.114 & 1.544 & 0.041\end{array}$

0.825

0.000

$0.000-0.000$

0.850

2.995

0.025

0.875

0.000

$0.000=0.000$

0.000

$0.000-0.000$

0.400

$\begin{array}{lll}2.817 & 1.118 & 0.015\end{array}$

0.900

0.425

2.585

0.923

0.009

0.925

2.309

0.744

0.006

0.950

0.975
0.000

0.000

0.000

0.000
$0.000-0.000$

$0.000-0.000$

$0.000-0.000$

$0.000-0.000$

$0.000-0.000$ 
Table 1. Energy Dissipation Function, $J(x)$, and Input Data - Continued

STOPPING POWER

$$
(\mathrm{dE} / \mathrm{dr})_{\mathrm{E}_{\mathrm{O}}}=1.29 \mathrm{Mev}\left(\mathrm{cm}^{2} / \mathrm{g}\right)
$$

RESIDUAL RANGE

$$
r_{0}=0.639 \mathrm{~g} / \mathrm{cm}^{2}
$$

\section{OTHER PARAYETERS}

\begin{tabular}{|c|c|c|c|}
\hline \multicolumn{4}{|c|}{ SCATTERING COEFFICIENTS } \\
\hline$\theta$ & $\sigma / \sigma_{R}$ & $\ell$ & $s_{\ell}$ \\
\hline $15^{\circ}$ & 1.066 & 1 & 3.93 \\
\hline & 1.092 & 2 & \\
\hline 45 & 1.072 & 3 & 20.2 \\
\hline 60 & 1.004 & 4 & 31.68 \\
\hline 75 & 0.896 & 5 & 44.92 \\
\hline 90 & 0.761 & 6 & 59.83 \\
\hline & 0.610 & 7 & 76.25 \\
\hline & 0.460 & 8 & 94.07 \\
\hline & 0.325 & 9 & 113.2 \\
\hline & 0.21 & 10 & 133.5 \\
\hline 6 & 0.150 & 11 & 155.0 \\
\hline & 126 & 12 & 177.5 \\
\hline
\end{tabular}

$$
\begin{array}{ll}
d_{1}=5.207 & A_{1}=0.4033 \\
\alpha=3.091 & A_{2}=0.8887 \\
A^{\text {PTI }}=3.576 & A_{3}=-0.4454 \\
A^{P L P}=4.087 & A_{4}=0.1534
\end{array}
$$

STOPPING PONER

$$
(\mathrm{dE} / \mathrm{dr})_{\mathrm{E}_{\mathrm{O}}}=1.30 \mathrm{Mev}\left(\mathrm{cm}^{2} / \mathrm{g}\right)
$$

RESIDUAL RANGE

$$
r_{0}=1.42 \mathrm{~g} / \mathrm{cm}^{2}
$$

CTHER PARAMETERS

$$
\begin{array}{ll}
\mathrm{d}_{1}=5.304 & \mathrm{~A}_{1}=0.3259 \\
\alpha=1.297 & \mathrm{~A}_{2}=0.9372 \\
\mathrm{~A}^{\text {PTI }}=3.153 & \mathrm{~A}_{3}=-0.4319 \\
\mathrm{~A}^{\mathrm{PLP}}=3.602 & \mathrm{~A}_{4}=0.1688
\end{array}
$$

\section{SCATTERING COEFFICIENTS}

$\begin{array}{cccc}\theta & \sigma / \sigma & l & s \ell \\ 15^{\circ} & 1.068 & 1 & 2.995 \\ 30 & 1.093 & 2 & 8.401 \\ 45 & 1.068 & 3 & 15.76 \\ 60 & 0.993 & 4 & 24.84 \\ 75 & 0.876 & 5 & 35.49 \\ 90 & 0.729 & 6 & 47.59 \\ 105 & 0.566 & 7 & 61.05 \\ 120 & 0.405 & 8 & 75.77 \\ 135 & 0.259 & 9 & 91.69 \\ 150 & 0.145 & 10 & 108.7 \\ 165 & 0.071 & 11 & 126.9 \\ 180 & 0.046 & 12 & 146.0\end{array}$

COPPER

$$
\mathrm{Z}=29, \mathrm{E}_{\mathrm{o}}=1.00 \mathrm{Mev}
$$

POINT PLANE ISOTROPIC PERPENDICULAR SOURCE

SOURCE$$
\begin{array}{llll}
x & \mathrm{~J}(\mathrm{x}) & \mathrm{J}(\mathrm{x}) & \mathrm{J}(-\mathrm{x})
\end{array}
$$

0.000

0.025

0.050

0.075

0.100

0.125

0.150

0.175

0.200

0.225

0.250

0.275

0.300

0.325

0.350

0.375

0.400

0.425

0.450

0.475
1.000

1.036

1.134

1.284

$$
2.320
$$

2.617

2.845

3.003

1.021

0.783

1.473

1.688

1.917

3.089

3.104

3.051

2.146

2.936

2.363

2.772

2.556

2.716

2.576

2.366

2.834

2.152

$2.904 \quad 1.937$

2.922

2.884

2.792

1.723

1.512

1.307

2.644

2.446

1.109

2.206

1.932
1.109
0.923
0.750

0.594
1.320
0.607

0.486

0.409

0.356

0.313

0.264

0.209

0.156

0.112

0.077

0.051

0.032

0.019

0.011

0.006

0.004

0.003 $x$

0.500

0.525

0.550

0.575

0.600

0.625

0.675

0.700

0.725

0.750

0.775

0.800

0.825

0.850

0.875

0.900

0.925

0.950

0.975
0.650

POINT

ISOTROPIC FERPENDICULAR SOURCE SOURCE

$\mathrm{J}(\mathrm{x}) \quad \mathrm{J}(\mathrm{x}) \quad \mathrm{J}(-\mathrm{x})$

$$
Z=29, E_{0}=2.00 \mathrm{Mev}
$$

$\begin{array}{cc}\text { POINT } & \text { PLANE } \\ \text { ISOTROPIC } & \text { PERPENDICULAR } \\ \text { SOURCE } & \text { SOURCE }\end{array}$

$\begin{array}{clll}x & J(x) & J(x) & J(-x) \\ 0.000 & 1.000 & 1.904 & 0.904 \\ 0.025 & 1.019 & 2.153 & 0.659 \\ 0.050 & 1.075 & 2.357 & 0.471 \\ 0.075 & 1.163 & 2.512 & 0.345 \\ & & & \\ 0.100 & 1.279 & 2.618 & 0.273 \\ 0.125 & 1.416 & 2.674 & 0.240 \\ 0.150 & 1.569 & 2.681 & 0.222 \\ 0.175 & 1.731 & 2.639 & 0.205\end{array}$

$\begin{array}{llll}0.200 & 1.894 & 2.550 & 0.179\end{array}$

0.225

0.250

2.051

2.195

2.423

0.144

0.275

2.317

2.268

0.104

0.300

2.411

2.098

0.069

$x$

0.325

2.469

1.919

0.043

0.350

2.486

1.746

0.024

0.375

2.457

1.365

0.013

0.007

0.400

2.382

0.425

$$
2.259
$$

1.184

0.450

2.093

1.008

0.003

0.003

0.8430 .003

0.475

1.890

0.688

0.003

0.900

1.638

0.456

0.003

1.339

0.337

0.002

1.050

0.239

0.002

0.784

0.162

0.001

0.552

0.103

0.001

0.363

0.061

0.000

0.219

$0.033-0.000$

0.119

$0.016-0.000$

0.056

$0.006-0.000$

0.022

$0.002-0.000$

0.007

$0.001-0.000$

0.002

$0.000-0.000$

0.000

$0.000-0.000$

0.000

$0.000-0.000$

0.000

$0.000-0.000$

0.000

$0.000-0.000$

0.000

$0.000-0.000$

0.000

$0.000-0.000$

0.000

$0.000-0.000$

0.000

$0.000-0.000$
POINTT

ISOTROPIC SOURCE
PLANE

PERPENDICULAR SOURCE

$\mathrm{J}(\mathrm{x}) \mathrm{J}(\mathrm{x}) \quad \mathrm{J}(-\mathrm{x})$

0.500

0.525

0.550

0.575

1.658

1.408

0.547

0.003

1.151

0.900

.421

0.312

0.002

0.221

0.001

0.600

0.625

0.668

0.465

0.300

0.175

$0.148-0.000$

$0.093-0.000$

0.67 ?

0.700

0.725

0.750

0.775

0.091

0.040

0.015 
Table 1. Energy Dissipation Function, $J(x)$, and Input Data - Continued

COPPER

$Z=29, E_{0}=4.00 \mathrm{Mev}$

STOPPING POHER

$$
(\mathrm{dE} / \mathrm{dr})_{\mathrm{E}_{0}}=1.36 \mathrm{Mev}\left(\mathrm{cm}^{2} / \mathrm{g}\right)
$$

RESIDUAI RANGE

$$
r_{0}=2.92 \mathrm{~g} / \mathrm{cm}^{2}
$$

OTHER PARAMETERS

$$
\begin{array}{ll}
d_{1}=5.567 & A_{1}=0.2836 \\
\alpha=0.5645 & A_{2}=0.8990 \\
{ }_{A}{ }^{\text {FII }}=2.596 & A_{3}=-0.3550 \\
{ }_{A} \text { PLP }=2.965 & A_{4}=0.1724
\end{array}
$$

SCATTERING COEFFICIENTS

$\begin{array}{cccc}\theta & \sigma / \sigma_{R} & \& & s_{\ell} \\ 15^{\circ} & 1.068 & 1 & 2.008 \\ 30 & 1.093 & 2 & 5.680 \\ 45 & 1.066 & 3 & 10.74 \\ 60 & 0.988 & 4 & 17.04 \\ 75 & 0.868 & 5 & 24.50 \\ 90 & 0.716 & 6 & 33.03 \\ 105 & 0.549 & 7 & 42.59 \\ 120 & 0.383 & 8 & 53.12 \\ 135 & 0.234 & 9 & 64.58 \\ 150 & 0.116 & 10 & 76.93 \\ 165 & 0.040 & 11 & 90.13 \\ 180 & 0.014 & 12 & 104.2\end{array}$

STOPPING PONER

$$
(\mathrm{dE} / \mathrm{dr})_{E_{0}}=1.45 \mathrm{Mev}\left(\mathrm{cm}^{2} / \mathrm{g}\right)
$$

RESIDUAL RANGE

$$
r_{0}=7.17 \mathrm{~g} / \mathrm{cm}^{2}
$$

OTHER PARAMETERS

$$
\begin{array}{ll}
d_{1}=6.060 & A_{1}=0.2252 \\
a^{P T I}=0.2020 & A_{2}=1.082 \\
A^{P T I}=2.348 & A_{3}=-0.7722 \\
A^{P L P}=2.043 & A_{4}=0.4649
\end{array}
$$

SCATTERING COEFFICIENTS

$\begin{array}{cccc}\theta & \sigma \not \sigma_{\mathrm{R}} & t & \mathrm{~s}_{\ell} \\ 15^{\circ} & 1.069 & 1 & 1.018 \\ 30 & 1.094 & 2 & 2.903 \\ 45 & 1.066 & 3 & 5.530 \\ 60 & 0.987 & 4 & 8.839 \\ 75 & 0.865 & 5 & 12.79 \\ 90 & 0.712 & 6 & 17.35 \\ 105 & 0.543 & 7 & 22.48 \\ 120 & 0.375 & 8 & 28.18 \\ 135 & 0.224 & 9 & 34.42 \\ 150 & 0.105 & 10 & 41.18 \\ 165 & 0.029 & 11 & 48.45 \\ 180 & 0.003 & 12 & 56.21\end{array}$

POINT PLANE

ISOTROPIC PERPENDICULAR SOURCE SOURCE

$\begin{array}{lll}x & J(x) & J(x)\end{array}$

0.000

0.025

0.050

0.075

0.100

0.125

0.150

0.175

$\mathrm{J}(\mathrm{x})$

$\mathrm{J}(\mathrm{x})$

1.000

1.407

0.407

1.007

1.546

1.032

1.732

0.313

1.074

1.947

0.259

0.233

1.130

2.151

1.201

2.296

0.215

1.284

2.357

0.181

1.378

2.358

0.083

0.200

1.481

2. 321

0.056

0.225

0.250

1.590

2.258

0.041

0.275

1.704

2.173

0.033

1.815

2.072

0.026

0.300

0.325

1.920

1.958

0.021

0.350

2.011

1.831

2.081

1.693

0.016

0.375

0.400

0.425

0.450

0.475

2.124

1.546

0.012

2.133

1.393

$2.106 \quad 1.236$

2.039

1.077
0.919

0.006

0.004

c.002

1.933

0.001

$Z=29, E_{0}=10.00 \mathrm{Mev}$

$\begin{array}{cc}\text { POINT } & \text { PLANE } \\ \text { ISOTROPIC } & \text { PERPENDICULAR } \\ \text { SOURCE } & \text { SOURCE }\end{array}$

$x$

$J(x)$

$\mathrm{J}(\mathrm{x}) \quad \mathrm{J}(-\mathrm{x})$

0.000

0.025

1.000

1.160

0.050

1.000

1.209

0.160

0.075

1.006

1.267

0.113

1.017

1.336

0.043

0.100

$1.033 \quad 1.420$

0.125

1.055

1.420

0.024

0.150

1.082

1.625

0.017

0.175

1.115

1.723

0.016

0.200

$\begin{array}{lll}1.153 & 1.803 & 0.020\end{array}$

0.225

1.197

0.250

1.247

1.864

1.903

0.020

0.275

1.302

1.920

0.019

1.362

1.914

0.300

1.426

1.88

0.012

0.325

1.494

1.832

0.007

$0.375 \quad 1.563$

$1.761-0.001$

0.400

1.631

$1.673-0.002$

0.425

1.694

$\begin{array}{lll}1.574 & -0.002\end{array}$

0.450

1.746
1.781

$\begin{array}{lll}1.464 & -0.002\end{array}$

$1.346-0.001$

POINT

ISOTROPIC PERPENDICULAR SOURCE SOURCE

$\mathrm{J}(\mathrm{x}) \quad \mathrm{J}(\mathrm{x}) \quad \mathrm{J}(-\mathrm{x})$

0.500

0.525

0.550

0.575

0.600

0.625

0.650

0.675

1.787

1.607

1.399

1.172

$0.767 \quad 0.001$

$\begin{array}{lll}0.623 & 0.000\end{array}$

$0.489-0.000$

$\begin{array}{llll}0.938 & 0.268 & -0.000\end{array}$

0.711

0.505

$0.183-0.000$

0.330

0.069

0.700

0.195

0.102

0.725
0.750

0.775

0.045

0.036

0.017

0.000

0.016

0.006

0.000

0.800

0.004

0.002

0.825

0.850

0.001

0.000

0.000

0.000

0.000

0.000

0.000

0.000

0.000

0.900

0.000

0.000

0.925

0.000

0.000

0.000

0.000

0.000

0.000

0.975

0.000

0.000

0.000

\section{POINT \\ ISOTROPIC SOURCE \\ PLANE}

$\mathrm{J}(\mathrm{x}) \quad \mathrm{J}(\mathrm{x}) \quad \mathrm{J}(-\mathrm{x})$

$\begin{array}{llll}0.500 & 1.792 & 1.219 & 0.000\end{array}$

0.525

0.550

0.575

1.771

1.086

0.001

1.712

0.947

0.001

1.612

0.805

0.001

0.600

1.468

0.663

0.001

0.625

1.284

0.525

0.001

0.650

1.068

0.395

0.000

0.675

0.835

0.280

0.000

0.700

0.606

$0.182-0.000$

0.725

0.401

$0.107-0.000$ 
STOPPING POWER

$$
(\mathrm{dE} / \mathrm{dr})_{\mathrm{F}_{0}}=3.55 \mathrm{Mov}\left(\mathrm{cm}^{2} / \mathrm{g}\right)
$$

RESIDUAL RANGE

$$
r_{0}=0.00840 \mathrm{~g} / \mathrm{cm}^{2}
$$

OTHER PARAMETERS

$$
\begin{array}{ll}
d_{1}=6.467 & A_{1}=0.5778 \\
a^{P}=-6.385 & A_{2}=1.618 \\
A^{P T I}=5.218 & A_{3}=-2.491 \\
A^{P L P}=5.913 & A_{4}=1.295
\end{array}
$$

SCATTERING COEFFICIENTS

$\begin{array}{cccc}\theta & \sigma / \sigma_{R} & l & s \ell \\ 15^{\circ} & 1.018 & 1 & 7.668 \\ 30 & 1.060 & 2 & 17.62 \\ 45 & 1.120 & 3 & 28.50 \\ 60 & 1.173 & 4 & 39.62 \\ 75 & 1.207 & 5 & 50.62 \\ 90 & 1.225 & 6 & 61.24 \\ 105 & 1.230 & 7 & 71.34 \\ 120 & 1.229 & 8 & 80.84 \\ 135 & 1.229 & 9 & 89.70 \\ 150 & 1.221 & 10 & 97.90 \\ 165 & 1.219 & 11 & 105.4 \\ 180 & 1.219 & 12 & 112.3\end{array}$

STOPPING POWER

$$
(\mathrm{dE} / \mathrm{dr})_{\mathrm{E}_{\mathrm{O}}}=2.29 \mathrm{Mev}\left(\mathrm{cm}^{2} / \mathrm{g}\right)
$$

RESIDUAL RANGE

$$
r_{0}=0.0265 \mathrm{~g} / \mathrm{cm}^{2}
$$

OTHER PARAMETERS

$$
\begin{array}{ll}
d_{1}=7.442 & A_{1}=0.6132 \\
a=-10.76 & A_{2}=1.161 \\
{ }_{A}^{P T I}=6.029 & A_{3}=-1.549 \\
A^{P L P}=6.865 & A_{4}=0.7748
\end{array}
$$

\section{SCATTERING COEFFICIENTS}

$\begin{array}{clcc}\theta & \sigma / \sigma_{\mathrm{R}} & l & \mathrm{~s}_{l} \\ 15^{\circ} & 1.115 & 1 & 8.205 \\ 30 & 1.223 & 2 & 20.01 \\ 45 & 1.284 & 3 & 33.87 \\ 60 & 1.277 & 4 & 49.01 \\ 75 & 1.197 & 5 & 64.94 \\ 90 & 1.055 & 6 & 81.33 \\ 105 & 0.869 & 7 & 97.89 \\ 120 & 0.664 & 8 & 114.4 \\ 135 & 0.467 & 9 & 130.8 \\ 150 & 0.305 & 10 & 146.9 \\ 165 & 0.198 & 11 & 162.6 \\ 180 & 0.161 & 12 & 177.8\end{array}$

TIN

$\mathrm{Z}=50, \mathrm{E}_{\mathrm{o}}=0.05 \mathrm{Mev}$

$\begin{array}{cc}\text { POINT } & \text { PLANE } \\ \text { ISOTROPIC } & \text { PERPENDICULAR } \\ \text { SOURCE } & \text { SOURCE }\end{array}$
SOURCE

$$
\mathrm{J}(\mathrm{x}) \quad \mathrm{J}(-\mathrm{x})
$$

$\stackrel{x}{0.000}$

0.025

0.050

0.075

0.100

0.125

0.150

0.175

0.200

0.225

0.250

0.275

0.300

0.325

0.350

0.375

0.400

0.425

0.450

0.475

\subsection{5}

2.995

1.167

2.096

4.704

1.686

$\begin{array}{lll}2.661 & 4.571 & 1.427 \\ 3.221 & 4.354 & 1.197 \\ 3.744 & 4.080 & 0.988 \\ 4.195 & 3.763 & 0.800\end{array}$

\section{$\begin{array}{lll}4.547 & 3.415 & 0.634\end{array}$}

$\begin{array}{lll}4.779 & 3.049 & 0.490\end{array}$

$\begin{array}{lll}4.879 & 2.677 & 0.369\end{array}$

4.842

2.310

0.270

$\begin{array}{lll}4.675 & 1.957 & 0.191\end{array}$

4.389

1.625

4.004

1.321

3.545

1.050

0.132

0.088

3.041

0.813

0.058

2.522

0.611

0.037

2.017

0.445

1.550

0.312

0.023

0.014

0.009
$4.491 \quad 2.424$

$\mathrm{Z}=50, \mathrm{E}_{0}=0.10 \mathrm{Mev}$

$\begin{array}{cc}\text { POINT } & \text { PLANE } \\ \text { ISOTROPIC } & \text { PERPENDICULAR } \\ \text { SOURCE } & \text { SOURCE }\end{array}$

$x$
0.000
0.025
0.050
0.075

$J(x)$

$\begin{array}{ll}J(x) & J(-x) \\ 4.087 & 3.087 \\ 4.593 & 2.539 \\ 4.879 & 2.075 \\ 4.895 & 1.711\end{array}$

0.100

0.125

0.150

0.175

1.000

1.173

1.603

2.180

4.895

2.815

3.436

$4.691 \quad 1.430$

3.992

4.37

1.194

4.446

4.035

3.672

0.981

0.200

0.225

4.771

3.295

0.786

0.250

4.951

2.908

0.612

4.980

2. 520

0.462

0.275

4.861

2.140

0.337

0.300

0.325

4.607

1.779

0.350

4.239

1.444

0.161

0.375

3.269

1.144

0.106

0.400

0.425

1.069

0.450

0.475
2.730

0.882

2.196

1.696

1.251

0.660

0.479

0.045

0.334

0.224

0.029

0.018

0.011

0.006

POINT

ISOTROPIC PERPENDICULAR SOURCE SOURCE

$x$

0.500

0.525

0.550

0.575

0.600

0.625

0.650

0.675

0.700

0.725

0.750

0.775

0.800

0.825

0.850

0.875

0.900

0.925

0.950

0.975
$\mathrm{J}(\mathrm{x})$

1.140

0.798

0.529

0.328

0.189

0.099

0.047

0.019

0.007

0.002

0.000

0.000

0.000

0.000

0.000

0.000

0.000

0.000

0.000

0.000
$\mathrm{J}(\mathrm{x}) \mathrm{J}(-\mathrm{x})$

0.2090 .005

$0.134 \quad 0.003$

0.0810 .001

$0.046 \quad 0.000$

$0.024 \quad 0.000$

$0.011-0.000$

$0.005-0.000$

$0.002-0.000$

$0.001-0.000$

$0.000-0.000$

$0.000-0.000$

$0.000-0.000$

$0.000-0.000$

$0.000-0.000$

$0.000-0.000$

$0.000=0.000$

$0.000-0.000$

$0.000-0.000$

$0.000-0.000$

$0.000-0.000$
POINT ISOTROPIC SOURCE

$\mathrm{J}(\mathrm{x})$

$x$
0.500

0.525

0.550

0.575

0.600

0.625

0.650

0.675

0.700

0.725

0.750

0.775

0.800

0.825

0.850

0.875

0.900

0.925

0.950

0.975

0.877

0.579

0.357

0.203

0.105

0.049

0.019

0.007

0.002

0.000

0.000 
Table 1. Energy Disaipation Function, $J(x)$, and Input Data - Continued

STOPPING POWER

$$
(d E / d r)_{E_{0}}=1.60 \mathrm{Mev}\left(\mathrm{cm}^{2} / \mathrm{g}\right)
$$

RESIDUAL RANGE

$$
r_{0}=0.0805 \mathrm{~g} / \mathrm{cm}^{2}
$$

OTHER PARAMETERS

$$
\begin{array}{ll}
d_{1}=8.129 & A_{1}=0.5730 \\
a=-35.95 & A_{2}=1.083 \\
A^{\text {PTI }}=6.512 & A_{3}=-1.237 \\
A^{\text {PLP }}=7.438 & A_{4}=0.5813
\end{array}
$$

SCATTERING COEPFICIENTS

$\begin{array}{cccc}\theta & \sigma / \sigma_{\mathrm{R}} & l & \mathrm{~s}_{\ell} \\ 15^{\circ} & 1.072 & 1 & 8.362 \\ 30 & 1.161 & 2 & 21.30 \\ 45 & 1.232 & 3 & 37.15 \\ 60 & 1.262 & 4 & 55.19 \\ 75 & 1.246 & 5 & 74.88 \\ 90 & 1.188 & 6 & 95.87 \\ 105 & 1.101 & 7 & 117.8 \\ 120 & 0.999 & 8 & 140.5 \\ 135 & 0.899 & 9 & 163.8 \\ 150 & 0.815 & 10 & 187.4 \\ 165 & 0.760 & 11 & 211.2 \\ 180 & 0.741 & 12 & 235.3\end{array}$

STOPPING POWER

$$
(d E / d r)_{E_{0}}=1.27 \mathrm{Nev}\left(\mathrm{cm}^{2} / 8\right)
$$

RESIDUAL RANGE

$$
r_{0}=0.225 \mathrm{~g} / \mathrm{cm}^{2}
$$

OTHER PARAMETERS

$$
\begin{array}{ll}
d_{1}=8.547 & A_{1}=0.4939 \\
a=16.16 & A_{2}=1.031 \\
A^{P I I}=6.636 & A_{3}=-0.8945 \\
A^{P L P}=7.582 & A_{4}=0.3696
\end{array}
$$

SCATTERING COEFFICIENTS

$\begin{array}{rlcc}\theta & \sigma / \sigma_{R} & l & s_{\ell} \\ 15^{\circ} & 1.096 & 1 & 8.049 \\ 30 & 1.197 & 2 & 21.18 \\ 45 & 1.264 & 3 & 37.77 \\ 60 & 1.276 & 4 & 57.15 \\ 75 & 1.227 & 5 & 78.83 \\ 90 & 1.126 & 6 & 102.5 \\ 105 & 0.988 & 7 & 127.8 \\ 120 & 0.832 & 8 & 154.5 \\ 135 & 0.682 & 9 & 182.4 \\ 150 & 0.557 & 10 & 211.4 \\ 165 & 0.475 & 11 & 241.2 \\ 180 & 0.446 & 12 & 271.9\end{array}$

TIN

$$
z=50, E_{0}=0.20 \mathrm{Mev}
$$

$\begin{array}{cc}\text { POINT } & \text { PLANE } \\ \text { ISOTROPIC } & \text { PERPENDICULAR } \\ \text { SOURCE } & \text { SOURCE }\end{array}$

$\begin{array}{llll}x & J(x) & J(x) & \mathrm{J}(-x)\end{array}$

0.000

1.000

4.076

3.076

0.025

0.050

1.180

4.637

2.401

1.617

4.791

1.948

0.075

2.192

4.704

1.616

0.100

0.125

0.150

0.175

\subsection{2}

4.491

1.342

3.408

4.204

1.101

3.932

3.869

0.888
0.702

$4.351 \quad 3.499$

0.200

0.225

4.641

3.111

0.542

4.786

2.715

0.409

4.780

2.326

0.299

0.275

4.628

1.952

0.211

\section{$\begin{array}{lll}4.343 & 1.602 & 0.144\end{array}$}

0.300

0.325
0.350

$3.949 \quad 1.28$

$\begin{array}{lll}3.474 & 1.002 & 0.058 \\ 2.952 & 0.759 & 0.034\end{array}$

0.094

0.375

0.400

0.425

2.417
1.900

0.450

1.430

0.557

1.024

0.394
0.267

0.019

0.010

0.173

0.002

$$
Z=50, E_{0}=0.40 \mathrm{Mev}
$$

POINT

ISOTROPIC PERPENDICULAR SOURCE SOURCE

$\begin{array}{clll}x & J(x) & J(x) & J(-x) \\ 0.000 & 1.000 & 3.724 & 2.724 \\ 0.025 & 1.156 & 4.264 & 2.104 \\ 0.050 & 1.543 & 4.447 & 1.693 \\ 0.075 & 2.058 & 4.395 & 1.402 \\ & & & \\ 0.100 & 2.620 & 4.200 & 1.168 \\ 0.125 & 3.161 & 3.920 & 0.961 \\ 0.150 & 3.633 & 3.590 & 0.773 \\ 0.175 & 4.000 & 3.228 & 0.604 \\ 0.200 & 4.241 & 2.852 & 0.459 \\ 0.225 & 4.347 & 2.745 & 0.337 \\ 0.250 & 4.318 & 2.108 & 0.240 \\ 0.275 & 4.162 & 1.760 & 0.164 \\ 0.300 & 3.893 & 1.439 & 0.109 \\ 0.325 & 3.530 & 1.150 & 0.070 \\ 0.350 & 3.098 & 0.896 & 0.044 \\ 0.375 & 2.625 & 0.678 & 0.027 \\ 0.400 & 2.141 & 0.496 & 0.016 \\ 0.425 & 1.675 & 0.349 & 0.009 \\ 0.450 & 1.252 & 0.235 & 0.005 \\ 0.475 & 0.889 & 0.151 & 0.003\end{array}$

$x$
0.500
0.525
0.550
0.575
0.600
0.625
0.650
0.675
0.700
0.725
0.750
0.775
0.800
0.825
0.850
0.875
0.900
0.925
0.950
0.975

$\begin{array}{lc}\text { POINT } & \text { PLANE } \\ \text { ISOTROPIC } & \text { PERPENDICULAR } \\ \text { SOURCE } & \text { SOURCE }\end{array}$

$x$
0.500
0.525
0.500
0.575

$\mathrm{J}(\mathrm{x})$

$J(x) \quad J(-x)$

0.694

0.441

0.108

0.061

0.261

0.142

0.016

0.001

0.001

0.600

0.625

0.650

0.070

0.030

0.011

0.007

0.003

0.001

$0.004 \quad 0.000$

0.000

0.700

0.725

0.750

0.001

0.000

0.000

0.000

0.000

0.000

0.000

0.000

0.000

0.800

0.825

0.850

0.875

0.900

0.925

0.950

0.975

0.000

0.000

0.000

0.000

0.000

0.000

0.000

0.000

0.000

0.000

0.000

0.000

0.000

0.000

0.000

0.000

0.000

0.000

0.000

0.000

0.000

0.000

$\theta .000$

0.000

0.000

0.000

0.000

0.000

$\begin{array}{cc}\text { POINT } & \text { POINT } \\ \text { ISOTROPIC } & \text { PERPENDICULAR } \\ \text { SOURCE } & \text { SOURCE }\end{array}$

$J(x) \quad J(x) \quad J(-x)$

$\begin{array}{lll}0.596 & 0.091 & 0.001\end{array}$

0.374

0.218

0.052

0.001

0.117

0.027

0.000

\subsection{6}

0.024

0.009

0.003

$0.006-0.060$

$0.002-0.000$

$0.001-0.000$

$0.000=0.000$

\section{$\begin{array}{llll}0.001 & 0.000 & -0.000\end{array}$}

$\begin{array}{llll}0.000 & 0.000 & -0.000\end{array}$

$\begin{array}{llll}0.000 & 0.000 & -0.000\end{array}$

0.000

$0.000=0.000$

$\begin{array}{llll}0.000 & 0.000 & -0.000\end{array}$

$\begin{array}{llll}0.000 & 0.000 & =0.000\end{array}$

$\begin{array}{llll}0.000 & 0.000 & -0.000\end{array}$

0.000

$0.000-0.000$

$\begin{array}{llll}0.000 & 0.000 & -0.000\end{array}$

$\begin{array}{llll}0.000 & 0.000 & -0.000\end{array}$

$\begin{array}{llll}0.000 & 0.000 & -0.000\end{array}$

0.000

$0.000-0.000$ 
STOPPING PONER

$$
(\mathrm{dE} / \mathrm{dr})_{\mathrm{E}_{\mathrm{O}}}=1.16 \mathrm{Mev}\left(\mathrm{cm}^{2} / \mathrm{g}\right)
$$

RESIDUAL RANGE

$$
r_{O}=0.475 \mathrm{~g} / \mathrm{cm}^{2}
$$

OTHER PARAMETERS

$$
\begin{array}{ll}
d_{1}=8.797 & A_{1}=0.4294 \\
a^{1}=5.083 & A_{2}=0.9362 \\
{ }^{\mathrm{PT}}=6.448 & A_{3}=-0.5806 \\
{ }_{A}{ }^{P L P}=7.361 & A_{4}=0.2150
\end{array}
$$

SCATTERING COEFFICIENTS

$\begin{array}{cccc}\theta & \sigma / \sigma_{\mathrm{R}} & \ell & \mathrm{s}_{\ell} \\ 15^{\circ} & 1.109 & 1 & 7.351 \\ 30 & 1.216 & 2 & 19.72 \\ 45 & 1.279 & 3 & 35.67 \\ 60 & 1.277 & 4 & 54.59 \\ 75 & 1.208 & 5 & 76.09 \\ 90 & 1.078 & 6 & 99.84 \\ 105 & 0.907 & 7 & 125.6 \\ 120 & 0.718 & 8 & 153.1 \\ 135 & 0.535 & 9 & 182.3 \\ 150 & 0.385 & 10 & 212.8 \\ 165 & 0.285 & 11 & 244.7 \\ 180 & 0.251 & 12 & 277.6\end{array}$

STOPPING POWER

$$
(\mathrm{dE} / \mathrm{dr})_{E_{O}}=1.13 \mathrm{Mev}\left(\mathrm{cm}^{2} / \mathrm{g}\right)
$$

RESIDUAL RANGE

$$
\begin{aligned}
& r_{O}=0.738 \mathrm{~g} / \mathrm{cm}^{2} \\
& \text { OTHER PARAMETERS } \\
& d_{1}=8.972 \quad A_{1}=0.3919 \\
& \alpha=2.920 \quad A_{2}=0.8830 \\
& \mathrm{~A}^{\mathrm{PTI}}=6.193 \quad \mathrm{~A}_{3}=-0.4221 \\
& { }_{A} P L P=7.056 \quad A_{4}=0.1472
\end{aligned}
$$

SCATTERING GNEFFICIENTS

$\begin{array}{cccc}\theta & \sigma / \sigma_{\mathrm{R}} & l & s_{\ell} \\ 15^{\circ} & 1.115 & 1 & 6.684 \\ 30 & 1.223 & 2 & 18.11 \\ 45 & 1.284 & 3 & 32.99 \\ 60 & 1.277 & 4 & 50.82 \\ 75 & 1.197 & 5 & 71.22 \\ 90 & 1.055 & 6 & 93.94 \\ 105 & 0.869 & 7 & 118.7 \\ 120 & 0.664 & 8 & 145.4 \\ 135 & 0.467 & 9 & 173.9 \\ 150 & 0.305 & 10 & 203.9 \\ 165 & 0.198 & 11 & 235.3 \\ 180 & 0.161 & 12 & 268.1\end{array}$

TIN

$$
\mathrm{Z}=50, \mathrm{E}_{\mathrm{O}}=0.70 \mathrm{Mev}
$$

$\begin{array}{cc}\text { POINT } & \text { PLANE } \\ \text { ISOTROPIC } & \text { PERPENDICULAR } \\ \text { SOURCE } & \text { SOURCE }\end{array}$

$\begin{array}{ccccc}x & J(x) & J(x) & J(-x) & x \\ 0.000 & 1.000 & 3.353 & 2.353 & 0.500 \\ 0.025 & 1.126 & 3.851 & 1.788 & 0.525 \\ 0.050 & 1.444 & 4.040 & 1.410 & 0.550 \\ 0.075 & 1.872 & 4.015 & 1.150 & 0.575 \\ 0.100 & 2.344 & 3.853 & 0.954 & 0.600 \\ 0.125 & 2.806 & 3.608 & 0.786 & 0.625 \\ 0.150 & 3.214 & 3.314 & 0.636 & 0.650 \\ 0.175 & 3.538 & 2.991 & 0.500 & 0.675 \\ 0.200 & 3.757 & 2.652 & 0.382 & 0.700 \\ 0.225 & 3.861 & 2.311 & 0.281 & 0.725 \\ 0.250 & 3.848 & 1.976 & 0.199 & 0.750 \\ 0.275 & 3.724 & 1.658 & 0.135 & 0.775 \\ & & & & \\ 0.300 & 3.500 & 1.363 & 0.088 & 0.800 \\ 0.325 & 3.193 & 1.095 & 0.055 & 0.825 \\ 0.350 & 2.823 & 0.858 & 0.033 & 0.850 \\ 0.375 & 2.413 & 0.654 & 0.019 & 0.875 \\ 0.400 & 1.988 & 0.483 & 0.012 & 0.900 \\ 0.425 & 1.573 & 0.344 & 0.007 & 0.925 \\ 0.450 & 1.189 & 0.235 & 0.004 & 0.950 \\ 0.475 & 0.855 & 0.153 & 0.003 & 0.975\end{array}$

$$
\mathrm{Z}=50, \mathrm{E}_{\mathrm{o}}=1.00 \mathrm{Mev}
$$

POINT PLANE

ISOTROPIC PERPENDICULAR SCURCE SOURCE

$\begin{array}{clllc}x & J(x) & J(x) & J(-x) & x \\ 0.000 & 1.000 & 3.031 & 2.031 & 0.500 \\ 0.025 & 1.103 & 3.418 & 1.619 & 0.525 \\ 0.050 & 1.367 & 3.673 & 1.273 & 0.550 \\ 0.075 & 1.729 & 3.765 & 1.003 & 0.575 \\ & & & & \\ 0.100 & 2.135 & 3.689 & 0.806 & 0.600 \\ 0.125 & 2.539 & 3.479 & 0.661 & 0.625 \\ 0.150 & 2.905 & 3.186 & 0.540 & 0.650 \\ 0.175 & 3.204 & 2.863 & 0.427 & 0.675 \\ 0.200 & 3.417 & 2.538 & 0.325 & 0.700 \\ 0.225 & 3.531 & 2.221 & 0.239 & 0.725 \\ 0.250 & 3.543 & 1.916 & 0.169 & 0.750 \\ 0.275 & 3.454 & 1.625 & 0.116 & 0.775 \\ & & & & \\ 0.300 & 3.273 & 1.353 & 0.076 & 0.800 \\ 0.325 & 3.013 & 1.102 & 0.048 & 0.825 \\ 0.350 & 2.691 & 0.875 & 0.029 & 0.850 \\ 0.375 & 2.326 & 0.675 & 0.018 & 0.875 \\ 0.400 & 1.942 & 0.504 & 0.010 & 0.900 \\ 0.425 & 1.558 & 0.362 & 0.006 & 0.925 \\ 0.450 & 1.197 & 0.250 & 0.004 & 0.950 \\ 0.475 & 0.876 & 0.165 & 0.002 & 0.975\end{array}$
$\begin{array}{cc}\text { POINT } & \text { PLANE } \\ \text { ISOTROPIC } & \text { PFRPENDICULAR } \\ \text { SOURCE } & \text { SOURCE }\end{array}$

$\begin{array}{llr}J(x) & J(x) & J(-x) \\ 0.580 & 0.094 & 0.002 \\ 0.370 & 0.054 & 0.001 \\ 0.218 & 0.029 & -0.000 \\ 0.118 & 0.014 & -0.000 \\ & & \\ 0.058 & 0.006 & -0.000 \\ 0.025 & 0.002 & -0.000 \\ 0.009 & 0.001 & -0.000 \\ 0.003 & 0.000 & -0.000 \\ & & \\ 0.001 & 0.000 & -0.000 \\ 0.000 & 0.000 & -0.000 \\ 0.000 & 0.000 & -0.000 \\ 0.000 & 0.000 & -0.000 \\ & & \\ 0.000 & 0.000 & -0.000 \\ 0.000 & 0.000 & -0.000 \\ 0.000 & 0.000 & -0.000 \\ 0.000 & 0.000 & -0.000 \\ & & \\ 0.000 & 0.000 & -0.000 \\ 0.000 & 0.000 & -0.000 \\ 0.000 & 0.000 & -0.000 \\ 0.000 & 0.000 & -0.000\end{array}$

POINT PLANE ISOTROPIC PERPENDICULAR SOURCE SOURCE

$\begin{array}{lll}J(x) & J(x) & J(-x) \\ 0.606 & 0.103 & 0.001 \\ 0.394 & 0.060 & 0.000 \\ 0.238 & 0.033 & 0.000 \\ 0.132 & 0.016 & -0.000 \\ & & \\ 0.066 & 0.007 & -0.000 \\ 0.029 & 0.003 & -0.000 \\ 0.011 & 0.001 & -0.000 \\ 0.004 & 0.000 & -0.000 \\ & & \\ 0.001 & 0.000 & -0.000 \\ 0.000 & 0.000 & -0.000 \\ 0.000 & 0.000 & -0.000 \\ 0.000 & 0.000 & -0.000 \\ & & \\ 0.000 & 0.000 & -0.000 \\ 0.000 & 0.000 & -0.000 \\ 0.000 & 0.000 & -0.000 \\ 0.000 & 0.000 & -0.000 \\ & & \\ 0.000 & 0.000 & -0.000 \\ 0.000 & 0.000 & -0.000 \\ 0.000 & 0.000 & -0.000 \\ 0.000 & 0.000 & -0.000\end{array}$


Table 1. Energy Dissipation Function, $J(x)$, and Input Data - Continued

TIN

$$
Z=50, E_{0}=2.00 \mathrm{Mev}
$$

STOPPING PCWER

$$
(d E / d r)_{E_{0}}=1.16 \mathrm{Mev}\left(\mathrm{cm}^{2} / \mathrm{g}\right)
$$

RESIDUAL RANGE

$$
I_{0}=1.61 \mathrm{~g} / \mathrm{cm}^{2}
$$

OTHER PARAYETERS

$$
\begin{array}{ll}
d_{1}=9.229 & A_{1}=0.3167 \\
a=1.201 & A_{2}=0.9157 \\
{ }_{A}^{\text {PTI }}=5.344 & A_{3}=-0.3981 \\
{ }_{A} \text { PLP }=6.068 & A_{4}=0.1658
\end{array}
$$

SCATTERING COEFFICIENTS

$\begin{array}{cccc}\theta & \sigma f_{R} & l & S_{l} \\ 15^{\circ} & 1.121 & 1 & 5.036 \\ 30 & 1.231 & 2 & 13.84 \\ 45 & 1.290 & 3 & 25.52 \\ 60 & 1.275 & 4 & 39.72 \\ 75 & 1.184 & 5 & 56.19 \\ 90 & 1.028 & 6 & 74.73 \\ 105 & 0.825 & 7 & 95.21 \\ 120 & 0.602 & 8 & 117.5 \\ 135 & 0.390 & 9 & 141.4 \\ 150 & 0.214 & 10 & 167.0 \\ 165 & 0.098 & 11 & 194.0 \\ 180 & 0.058 & 12 & 222.4\end{array}$

$\begin{array}{cc}\text { POINT } & \text { PLANE } \\ \text { ISOTROPIC } & \text { PERPENDICULAR } \\ \text { SOURCE } & \text { SOURCE }\end{array}$

$\begin{array}{clll}x & J(x) & J(x) & J(-x) \\ 0.000 & 1.000 & 2.493 & 1.493 \\ 0.025 & 1.055 & 2.815 & 1.155 \\ 0.050 & 1.209 & 3.045 & 0.876 \\ 0.075 & 1.436 & 3.170 & 0.661 \\ & & & \\ 0.100 & 1.706 & 3.184 & 0.510 \\ 0.125 & 1.993 & 3.098 & 0.412 \\ 0.150 & 2.271 & 2.933 & 0.348 \\ 0.175 & 2.522 & 2.716 & 0.295 \\ 0.200 & 2.726 & 2.470 & 0.242 \\ 0.225 & 2.872 & 2.211 & 0.186 \\ 0.250 & 2.950 & 1.948 & 0.132 \\ 0.275 & 2.954 & 1.690 & 0.087 \\ 0.300 & 2.884 & 1.442 & 0.052 \\ 0.325 & 2.743 & 1.209 & 0.029 \\ 0.350 & 2.539 & 0.994 & 0.015 \\ 0.375 & 2.282 & 0.798 & 0.008 \\ 0.400 & 1.988 & 0.624 & 0.005 \\ 0.425 & 1.673 & 0.472 & 0.004 \\ 0.450 & 1.355 & 0.345 & 0.004 \\ 0.475 & 1.050 & 0.241 & 0.003\end{array}$

$x$
0.500
0.525
0.550
0.575
0.600
0.625
0.650
0.675
0.700
0.725
0.750
0.775
0.800
0.825
0.850
0.875
0.900
0.925
0.950
0.975

$Z=50, E_{0}=4.00 \mathrm{Kev}$

STOPPING PONER

$$
(\mathrm{dE} / \mathrm{dr})_{E_{\circ}}=1.23 \mathrm{Nev}\left(\mathrm{cm}^{2} / \mathrm{g}\right)
$$

RESIDUAL RANGE

$$
r_{0}=3.29 \mathrm{~g} / \mathrm{cm}^{2}
$$

OTHER PARANETERS

$$
\begin{array}{ll}
d_{1}=9.905 & A_{1}=0.2757 \\
a=0.5076 & A_{2}=0.8755 \\
A^{\text {PII }}=4.281 & A_{3}=-0.3534 \\
A^{\text {PLP }}=4.558 & A_{4}=0.2022
\end{array}
$$

SCATTERING COEFFICIENTS

$\begin{array}{cccc}\theta & \sigma / \sigma_{R} & l & s_{\ell} \\ 15^{\circ} & 1.123 & 1 & 3.335 \\ 30 & 1.234 & 2 & 9.263 \\ 45 & 1.292 & 3 & 17.24 \\ 60 & 1.274 & 4 & 27.06 \\ 75 & 1.179 & 5 & 38.57 \\ 90 & 1.017 & 6 & 51.65 \\ 105 & 0.807 & 7 & 66.22 \\ 120 & 0.578 & 8 & 82.20 \\ 135 & 0.359 & 9 & 99.51 \\ 150 & 0.178 & 10 & 118.1 \\ 165 & 0.059 & 11 & 137.9 \\ 180 & 0.018 & 12 & 158.9\end{array}$

$\begin{array}{cc}\text { POINT } & \text { PLANE } \\ \text { ISOTROPIC } & \text { PERPENDICULAR } \\ \text { SOURCE } & \text { SOURCE }\end{array}$

$\begin{array}{clll}x & J(x) & J(x) & J(-x) \\ 0.000 & 1.000 & 1.895 & 0.895 \\ 0.025 & 1.024 & 2.037 & 0.751 \\ 0.050 & 1.096 & 2.221 & 0.606 \\ 0.075 & 1.207 & 2.424 & 0.443 \\ 0.100 & 1.349 & 2.613 & 0.294 \\ 0.125 & 1.513 & 2.744 & 0.185 \\ 0.150 & 1.690 & 2.776 & 0.136 \\ 0.175 & 1.871 & 2.697 & 0.137 \\ 0.200 & 2.046 & 2.537 & 0.149 \\ 0.225 & 2.204 & 2.334 & 0.139 \\ 0.250 & 2.336 & 2.108 & 0.111 \\ 0.275 & 2.430 & 1.878 & 0.079 \\ 0.300 & 2.478 & 1.653 & 0.051 \\ 0.325 & 2.472 & 1.436 & 0.029 \\ 0.350 & 2.411 & 1.231 & 0.013 \\ 0.375 & 2.293 & 1.038 & 0.002 \\ 0.400 & 2.124 & 0.861 & -0.002 \\ 0.425 & 1.911 & 0.699 & -0.004 \\ 0.450 & 1.665 & 0.553 & -0.003 \\ 0.475 & 1.400 & 0.424 & -0.002\end{array}$

$\begin{array}{cc}\text { POINT } & \text { PLANE } \\ \text { SOTROPIC } & \text { PERPENDICULAR } \\ \text { SOURCE } & \text { SOURCE }\end{array}$

$\begin{array}{llr}J(x) & J(x) & J(-x) \\ 0.775 & 0.160 & 0.002 \\ 0.540 & 0.100 & 0.001 \\ 0.352 & 0.059 & 0.000 \\ 0.212 & 0.032 & -0.000 \\ & & \\ 0.117 & 0.016 & -0.000 \\ 0.058 & 0.007 & -0.000 \\ 0.025 & 0.003 & -0.000 \\ 0.009 & 0.001 & -0.000 \\ & & \\ 0.003 & 0.000 & -0.000 \\ 0.001 & 0.000 & -0.000 \\ 0.000 & 0.000 & -0.000 \\ 0.000 & 0.000 & -0.000 \\ & & \\ 0.000 & 0.000 & -0.000 \\ 0.000 & 0.000 & -0.000 \\ 0.000 & 0.000 & -0.000 \\ 0.000 & 0.000 & -0.000 \\ & & \\ 0.000 & 0.000 & -0.000 \\ 0.000 & 0.000 & -0.000 \\ 0.000 & 0.000 & -0.000 \\ 0.000 & 0.000 & -0.000\end{array}$

\section{POINT PLANE ISOTROPIC PERPENDICULAR SOURCE SOURCE}

$\begin{array}{lll}J(x) & J(x) & J(-x) \\ 1.130 & 0.313 & -0.002 \\ 0.871 & 0.221 & -0.001 \\ 0.635 & 0.148 & -0.000 \\ 0.434 & 0.093 & -0.000 \\ 0.275 & 0.054 & 0.000 \\ 0.158 & 0.029 & 0.000 \\ 0.081 & 0.014 & 0.000 \\ 0.036 & 0.006 & 0.000 \\ & & \\ 0.013 & 0.002 & 0.000 \\ 0.004 & 0.001 & 0.000 \\ 0.001 & 0.000 & 0.000 \\ 0.000 & 0.000 & 0.000 \\ & & \\ 0.000 & 0.000 & 0.000 \\ -0.000 & 0.000 & 0.000 \\ -0.000 & 0.000 & 0.000 \\ -0.000 & 0.000 & 0.000 \\ & & \\ -0.000 & 0.000 & 0.000 \\ -0.000 & 0.000 & 0.000 \\ -0.000 & 0.000 & 0.000 \\ -0.000 & 0.000 & 0.000\end{array}$


Table 1. Energy Dissipation Function, $J(x)$, and Input Data - Continued

TIN

$Z=50, E_{0}=10.00 \mathrm{Mev}$

STOPPING POWER

$$
(d E / d r)_{E_{0}}=1.33 \operatorname{Mov}\left(\mathrm{cm}^{2} / \mathrm{g}\right)
$$

$\begin{array}{cc}\text { POINT } & \text { PLANE } \\ \text { ISOTROPIC } & \text { PERPENDICULAR } \\ \text { SOURCE } & \text { SOTRCE }\end{array}$

$\begin{array}{cc}\text { POINT } & \text { PLANE } \\ \text { ISOTROPIC } & \text { PERPENDICULAR } \\ \text { SOURCE } & \text { SOURCE }\end{array}$

RESIDUAL RANGE

$$
r_{0}=7.95 \mathrm{~g} / \mathrm{cm}^{2}
$$

OTHER PARAMETERS

$$
\begin{array}{ll}
d_{1}=11.50 & A_{1}=0.1902 \\
\alpha^{P T I}=0.1689 & A_{2}=1.295 \\
A^{P T I}=2.801 & A_{3}=-1.267 \\
A^{\text {PLP }}=3.166 & A_{4}=0.7827
\end{array}
$$

\section{SCATTERING COEFEICIENTS}

$\begin{array}{clcc}\theta & \sigma / \sigma_{R} & l & s_{\ell} \\ 15^{\circ} & 1.124 & 1 & 1.662 \\ 30 & 1.235 & 2 & 4.616 \\ 45 & 1.292 & 3 & 8.592 \\ 60 & 1.274 & 4 & 13.48 \\ 75 & 1.177 & 5 & 19.22 \\ 90 & 1.013 & 6 & 25.74 \\ 105 & 0.801 & 7 & 33.00 \\ 120 & 0.569 & 8 & 40.96 \\ 135 & 0.348 & 9 & 49.59 \\ 150 & 0.165 & 10 & 58.85 \\ 165 & 0.045 & 11 & 68.72 \\ 180 & 0.003 & 12 & 79.18\end{array}$

$\begin{array}{cccr}x & J(x) & J(x) & J(-x) \\ 0.000 & 1.000 & 1.436 & 0.436 \\ 0.025 & 1.004 & 1.545 & 0.328 \\ 0.050 & 1.020 & 1.668 & 0.209 \\ 0.075 & 1.049 & 1.794 & 0.097 \\ 0.100 & 1.091 & 1.902 & 0.013 \\ 0.125 & 1.146 & 1.973 & -0.022 \\ 0.150 & 1.215 & 2.011 & -0.012 \\ 0.175 & 1.294 & 2.036 & 0.008 \\ & & & \\ 0.200 & 1.382 & 2.053 & 0.026 \\ 0.225 & 1.476 & 2.060 & 0.040 \\ 0.250 & 1.574 & 2.050 & 0.050 \\ 0.275 & 1.671 & 2.018 & 0.055 \\ 0.300 & 1.764 & 1.962 & 0.053 \\ 0.325 & 1.848 & 1.876 & 0.045 \\ 0.350 & 1.917 & 1.760 & 0.032 \\ 0.375 & 1.966 & 1.618 & 0.017 \\ & & & \\ 0.400 & 1.988 & 1.454 & 0.004 \\ 0.425 & 1.978 & 1.278 & -0.005 \\ 0.450 & 1.930 & 1.098 & -0.009 \\ 0.475 & 1.842 & 0.923 & -0.009\end{array}$

$x$
0.500
0.525
0.550
0.575
0.600
0.625
0.650
0.675
0.700
0.725
0.750
0.775
0.800
0.825
0.850
0.875
0.900
0.925
0.950
0.975

$J(x)$

1.712

1.541

1.335

1.105

0.864

0.632

0.424

0.258

0.139

0.065

0.026

0.008

$J(x)$

0.759

0.608

0.472

0.354

0.254

0.173

0.110

$\mathrm{J}(-\mathrm{x})$

$-0.007$

$-0.006$

$-0.004$

$-0.002$

$-0.001$

$-0.000$

0.000

0.000

0.034

0.015

0.000

0.000

0.000

0.000

$0.002-0.000$

$0.000-0.000$

0.000

0.000

$0.000-0.000$

0.000

$\begin{array}{lll}0.000 & -0.000\end{array}$

0.000

$0.000-0.000$

0.000

$0.000-0.000$

0.000

$0.000-0.000$

$0.000-0.000$

0.000

0.000 
$Z=82, E_{0}=0.10 \mathrm{Mev}$

STOPPING POWER

$$
(\mathrm{dE} / \mathrm{dr})_{\mathrm{E}_{0}}=1.97 \mathrm{Mev}\left(\mathrm{cm}^{2} / \mathrm{g}\right)
$$

RESIDUAL RANGE

$$
r_{0}=0.0313 \mathrm{~g} / \mathrm{cm}^{2}
$$

OTHER PARAMETERS

$$
a_{1}=10.90 \quad A_{1}=0.5442
$$

$\alpha=-5.084 \quad A_{2}=1.1310$

${ }_{A} P T I=9.759 \quad A_{3}=-1.552$

$A^{P L P}=11.18 \quad A_{4}=0.6977$

SCATTERING COEFFICIENTS

$\begin{array}{clcc}\theta & \sigma \sigma_{\mathrm{R}} & l & s \ell \\ 15^{\circ} & 1.074 & 1 & 13.56 \\ 30 & 1.230 & 2 & 29.52 \\ 45 & 1.479 & 3 & 46.58 \\ 60 & 1.728 & 4 & 64.12 \\ 75 & 1.896 & 5 & 81.67 \\ 90 & 1.936 & 6 & 98.84 \\ 105 & 1.842 & 7 & 115.4 \\ 120 & 1.640 & 8 & 131.1 \\ 135 & 1.385 & 9 & 145.8 \\ 150 & 1.143 & 10 & 159.6 \\ 165 & 0.969 & 11 & 172.4 \\ 180 & 0.908 & 12 & 184.7\end{array}$

STOPPING POWER

$$
(\mathrm{dE} / \mathrm{dr})_{E_{0}}=1.02 \mathrm{Mov}\left(\mathrm{cm}^{2} / \mathrm{g}\right)
$$

RESIDUAL RANGE

$$
r_{0}=0.544 \mathrm{~g} / \mathrm{cm}^{2}
$$

OTHER PARANETERS

$$
\begin{array}{ll}
d_{1}=15.90 & A_{1}=0.4133 \\
\alpha=6.172 & A_{2}=0.9603 \\
A^{\text {PTI }}=12.80 & A_{3}=-0.5993 \\
A^{\text {PLP }}=14.82 & A_{4}=0.2256
\end{array}
$$

SCATTERING COEFFICIENTS

$\begin{array}{cccc}\theta & \sigma / \sigma_{\mathrm{R}} & \ell & s_{\ell} \\ 15^{\circ} & 1.098 & 1 & 13.68 \\ 30 & 1.290 & 2 & 34.41 \\ 45 & 1.564 & 3 & 59.30 \\ 60 & 1.819 & 4 & 87.92 \\ 75 & 1.971 & 5 & 119.8 \\ 90 & 1.966 & 6 & 154.5 \\ 105 & 1.801 & 7 & 191.6 \\ 120 & 1.510 & 8 & 230.8 \\ 135 & 1.159 & 9 & 271.9 \\ 150 & 0.830 & 10 & 314.5 \\ 165 & 0.595 & 11 & 358.5 \\ 180 & 0.511 & 12 & 403.5\end{array}$

1.393

$\begin{array}{cc}\text { POINT } & \text { PLANE } \\ \text { SOTROPIC } & \text { PERPENDICULAR } \\ \text { SOURCE } & \text { SOURCE }\end{array}$

$\begin{array}{clll}x & J(x) & J(x) & J(-x) \\ 0.000 & 1.000 & 5.264 & 4.264 \\ 0.025 & 1.470 & 5.834 & 3.439 \\ 0.050 & 2.374 & 5.822 & 2.816 \\ 0.075 & 3.384 & 5.490 & 2.304 \\ & & & \\ 0.100 & 4.348 & 5.000 & 1.858 \\ 0.125 & 5.149 & 4.430 & 1.466 \\ 0.150 & 5.708 & 3.838 & 1.126 \\ 0.175 & 5.986 & 3.249 & 0.841 \\ 0.200 & 5.980 & 2.688 & 0.608 \\ 0.225 & 5.713 & 2.168 & 0.427 \\ 0.250 & 5.232 & 1.704 & 0.289 \\ 0.275 & 4.597 & 1.301 & 0.189 \\ 0.300 & 3.874 & 0.963 & 0.119 \\ 0.325 & 3.128 & 0.680 & 0.072 \\ 0.350 & 2.415 & 0.475 & 0.042 \\ 0.375 & 1.778 & 0.314 & 0.023 \\ 0.400 & 1.244 & 0.198 & 0.012 \\ 0.425 & 0.824 & 0.119 & 0.006 \\ 0.450 & 0.514 & 0.068 & 0.003 \\ 0.475 & 0.300 & 0.036 & 0.001\end{array}$

$$
Z=82, E_{0}=0.70 \mathrm{Mev}
$$

$\begin{array}{cc}\text { POINT } & \text { PLANE } \\ \text { ISOTROPIC } & \text { PERPENDICULAR } \\ \text { SOURCE } & \text { SOURCE }\end{array}$

$x$

0.000

0.025

0.050

0.075

0.100

0.125

0.150

C. 175

0.200

0.225

0.250

0.275

0.300

0.325

0.350

0.375

0.400

0.425

0.450

0.475

$$
\mathrm{J}(\mathrm{x})
$$

1.000

2.236

3.176

4.005

4.615

4.956

5.023

4.836

4.438

3.887

$$
\mathrm{J}(\mathrm{x})
$$

4.576

5.105

5.068

4.708

$\mathrm{J}(-\mathrm{x})$

3.576

2.789

2.194

1.722

4.187

3.603

1.336

1.336
1.015

3.016

2.462

0.749

0.533

1.958

1.515

0.365
0.239

1.136

0.823

1.149

2.579

1.946

0.573

0.381

1.389

$0.93 / 4$

$0.2 / 1$

0.144

0.050

0.027

0.014

0.588

0.345

0.081

0.043

0.187

0.093
0.021

0.009
0.003

0.002

0.001

0.000 $x$
0.500

0.525

0.550

0.575

0.600

0.625

0.650

0.675

0.700

0.725

0.750

0.775

0.800

0.825

0.850

0.875

0.900

0.925

0.950

0.975
POINT

ISOTROPIC PERPENDICULAR SOURCE SOURCE

$J(x) \quad J(x) \quad J(-x)$

$\begin{array}{lll}0.163 & 0.018 & 0.001\end{array}$

$\begin{array}{lll}0.081 & 0.008 & 0.000\end{array}$

$\begin{array}{lll}0.037 & 0.003 & 0.000\end{array}$

$\begin{array}{llll}0.015 & 0.001 & -0.000\end{array}$

$\begin{array}{llll}0.005 & 0.000 & -0.000\end{array}$

$\begin{array}{llll}0.002 & 0.000 & -0.000\end{array}$

$\begin{array}{llll}0.000 & 0.000 & -0.000\end{array}$

0.000

$0.000-0.000$

$\begin{array}{llll}0.000 & 0.000 & -0.000\end{array}$

$\begin{array}{llll}0.000 & 0.000 & -0.000\end{array}$

0.000

$0.000-0.000$

0.000

$0.000=0.000$

$\begin{array}{llll}0.000 & 0.000 & -0.000\end{array}$

0.000

$0.000-0.000$

0.000

$0.000 \quad-0.000$

0.000

$0.000-0.000$

0.000

$0.000-0.000$

0.000

$0.000-0.000$

0.000

$0.000-0.000$

$-0.001$

$0.000-0.000$

\section{POINT PLANE ISOTROPIC PERPENDICULAR SOURCE SOURCE}

$x$
0.500
0.525
0.550
0.575

0.600

0.625

0.650

0.675

$J(x)$

$J(x) \quad J(-x)$

0.042

0.017

0.006

0.002

$0.004 \quad 0.000$

$0.001-0.000$

$0.000-0.000$

$0.000-0.000$

$\begin{array}{llll}0.000 & 0.000 & -0.000\end{array}$

$\begin{array}{llll}0.000 & 0.000 & -0.000\end{array}$

0.000

0.000

$0.000-0.000$

0.700

0.725

0.000

$0.000-0.000$

0.750

0.000

$0.000-0.000$

0.775

0.000

$0.000-0.000$

0.000

$0.000-0.000$

0.800

0.825

0.000

$0.000-0.000$

0.850

0.875

0.000

$0.000-0.000$

0.000

$0.000-0.000$

0.000

$0.000=0.000$

0.900

0.925

0.000

$0.000-0.000$

0.950

$-0.000$

$0.000-0.000$

$0.000-0.000$

0.975

$-0.000$

$0.000-0.000$

$-0.000$

$0.000-0.000$ 
Table 1. Energy Dissipation Function, $J(x)$, and Input Data - Continued

LEAD

$$
\mathrm{Z}=82, \mathrm{E}_{\mathrm{O}}=1.00 \mathrm{Mev}
$$

STOPPING PONER

$$
(\mathrm{dE} / \mathrm{dr})_{E_{O}}=1.01 \mathrm{Mev}\left(\mathrm{cm}^{2} / \mathrm{g}\right)
$$

RESIDUAL RANGE

$$
r_{0}=0.841 \mathrm{~g} / \mathrm{cm}^{2}
$$

OTHER PARAMETERS

$$
\begin{array}{ll}
\mathrm{d}_{1}=16.75 & \mathrm{~A}_{1}=0.3810 \\
\alpha=2.890 & \mathrm{~A}_{2}=0.9006 \\
\mathrm{~A}^{\mathrm{PTI}}=12.34 & \mathrm{~A}_{3}=-0.4442 \\
\mathrm{~A}^{\mathrm{PLP}}=14.23 & \mathrm{~A}_{4}=0.1625
\end{array}
$$

SCATTERING COEFFICIENTS

$\begin{array}{cccc}\theta & \sigma / \sigma_{\mathrm{R}} & \ell & \mathrm{s}_{\ell} \\ 15^{\circ} & 1.108 & 1 & 12.44 \\ 30 & 1.315 & 2 & 31.72 \\ 45 & 1.599 & 3 & 55.21 \\ 60 & 1.857 & 4 & 82.51 \\ 76 & 2.000 & 5 & 113.2 \\ 90 & 1.975 & 6 & 147.0 \\ 105 & 1.777 & 7 & 183.4 \\ 120 & 1.444 & 8 & 222.2 \\ 135 & 1.050 & 9 & 263.2 \\ 150 & 0.683 & 10 & 306.1 \\ 165 & 0.422 & 11 & 350.7 \\ 180 & 0.328 & 12 & 396.9\end{array}$

STOPPING PONER

$$
(\mathrm{dE} / \mathrm{dr})_{\mathrm{E}_{\mathrm{O}}}=1.04 \mathrm{Mev}\left(\mathrm{cm}^{2} / \mathrm{g}\right)
$$

RESIDUAL RANGE

$$
r_{O}=1.82 \mathrm{~g} / \mathrm{cm}^{2}
$$

\section{OTHER PARAMETERS}

$$
\begin{array}{ll}
\mathrm{d}_{1}=17.46 & \mathrm{~A}_{1}=0.3433 \\
{ }=1.144 & \mathrm{~A}_{2}=0.4377 \\
{ }_{\mathrm{ATI}}=10.26 & \mathrm{~A}_{3}=0.6620 \\
{ }_{A}^{\mathrm{PLP}}=11.72 & \mathrm{~A}_{4}=-0.4430
\end{array}
$$

SCATTERING COEFFICIENTS

$\begin{array}{cccc}\theta & \sigma / \sigma_{\mathrm{R}} & l & \mathrm{~S}_{\ell} \\ 15^{\circ} & 1.120 & 1 & 9.317 \\ 30 & 1.334 & 2 & 24.27 \\ 45 & 1.638 & 3 & 42.94 \\ 60 & 1.897 & 4 & 65.09 \\ 75 & 2.029 & 5 & 90.42 \\ 90 & 1.979 & 6 & 118.7 \\ 105 & 1.745 & 7 & 149.6 \\ 120 & 1.366 & 8 & 183.1 \\ 135 & 0.924 & 9 & 218.8 \\ 150 & 0.513 & 10 & 256.7 \\ 165 & 0.222 & 11 & 296.5 \\ 180 & 0.119 & 12 & 338.1\end{array}$

$\begin{array}{cc}\text { POINT } & \text { PLANE } \\ \text { ISOTROPIC } & \text { PERPENDICULAR } \\ \text { SOURCE } & \text { SOURCE }\end{array}$

$\begin{array}{clll}x & J(x) & J(x) & J(-x) \\ 0.000 & 1.000 & 4.208 & 3.208 \\ 0.025 & 1.329 & 4.730 & 2.469 \\ 0.050 & 2.053 & 4.736 & 1.927 \\ 0.075 & 2.880 & 4.438 & 1.509 \\ & & & \\ 0.100 & 3.629 & 3.978 & 1.172 \\ 0.125 & 4.195 & 3.451 & 0.893 \\ 0.150 & 4.530 & 2.913 & 0.662 \\ 0.175 & 4.621 & 2.398 & 0.474 \\ & & & \\ 0.200 & 4.484 & 1.924 & 0.325 \\ 0.225 & 4.153 & 1.503 & 0.213 \\ 0.250 & 3.675 & 1.140 & 0.133 \\ 0.275 & 3.107 & 0.835 & 0.079 \\ & & & \\ 0.300 & 2.504 & 0.589 & 0.044 \\ 0.325 & 1.918 & 0.398 & 0.024 \\ 0.350 & 1.392 & 0.256 & 0.013 \\ 0.375 & 0.953 & 0.156 & 0.006 \\ 0.400 & 0.611 & 0.089 & 0.003 \\ 0.425 & 0.365 & 0.048 & 0.002 \\ 0.450 & 0.209 & 0.024 & 0.001 \\ 0.475 & 0.102 & 0.011 & 0.000\end{array}$

$$
\mathrm{Z}=82, \mathrm{E}_{\mathrm{O}}=2.00 \mathrm{Mev}
$$

$\begin{array}{cc}\text { POINT } & \text { PLANE } \\ \text { ISOTROPIC } & \text { PERPENDICULAR } \\ \text { SOURCE } & \text { SOURCE }\end{array}$

$\begin{array}{clllllll}x & J(x) & J(x) & J(-x) & x & J(x) & J(x) & J(-x) \\ 0.000 & 1.000 & 3.412 & 2.412 & 0.500 & 0.097 & 0.011 & 0.000 \\ 0.025 & 1.199 & 3.971 & 1.745 & 0.525 & 0.045 & 0.005 & -0.000 \\ 0.050 & 1.663 & 4.070 & 1.352 & 0.550 & 0.019 & 0.002 & -0.000 \\ 0.075 & 2.232 & 3.902 & 1.090 & 0.575 & 0.007 & 0.001 & -0.000 \\ & & & & & & & \\ 0.100 & 2.792 & 3.596 & 0.875 & 0.600 & 0.002 & 0.000 & -0.000 \\ 0.125 & 3.265 & 3.224 & 0.682 & 0.625 & 0.001 & 0.000 & -0.000 \\ 0.150 & 3.603 & 2.817 & 0.511 & 0.650 & 0.000 & 0.000 & -0.000 \\ 0.175 & 3.780 & 2.403 & 0.369 & 0.675 & 0.000 & 0.000 & -0.000 \\ & & & & & & & \\ 0.200 & 3.792 & 2.000 & 0.255 & 0.700 & 0.000 & 0.000 & -0.000 \\ 0.225 & 3.647 & 1.623 & 0.169 & 0.725 & 0.000 & 0.000 & -0.000 \\ 0.250 & 3.368 & 1.283 & 0.107 & 0.750 & 0.000 & 0.000 & -0.000 \\ 0.275 & 2.987 & 0.985 & 0.066 & 0.775 & 0.000 & 0.000 & -0.000 \\ & & & & & & & \\ 0.300 & 2.540 & 0.733 & 0.039 & 0.800 & 0.000 & 0.000 & -0.000 \\ 0.325 & 2.067 & 0.527 & 0.023 & 0.825 & 0.000 & 0.000 & -0.000 \\ 0.350 & 1.606 & 0.363 & 0.013 & 0.850 & 0.000 & 0.000 & -0.000 \\ 0.375 & 1.185 & 0.240 & 0.007 & 0.875 & 0.000 & 0.000 & -0.000 \\ 0.400 & 0.827 & 0.150 & 0.004 & 0.900 & 0.000 & 0.000 & -0.000 \\ 0.425 & 0.543 & 0.088 & 0.002 & 0.925 & 0.000 & 0.000 & -0.000 \\ 0.450 & 0.333 & 0.048 & 0.001 & 0.950 & 0.000 & 0.000 & -0.000 \\ 0.475 & 0.188 & 0.025 & 0.000 & 0.975 & 0.000 & 0.000 & -0.000\end{array}$

POIN"m PLANE ISOTROPIC PERPENDICULAR SOURCE SOURCE

$x$
0.500
0.525
0.550
0.575

$\mathrm{J}(\mathrm{x})$

$\mathrm{J}(\mathrm{x}) \quad \mathrm{J}(-\mathrm{x})$

0.047

0.004

0.000

0.019

$0.002-0.000$

0.007

$0.001-0.000$

0.600

0.625

0.650

0.675

0.700

0.725

0.750

0.775

0.800

0.825

0.850

0.875

0.900

0.925

0.950

0.975
$0.000-0.000$

$\begin{array}{lll}0.001 & 0.000 & -0.000\end{array}$

$\begin{array}{llll}0.000 & 0.000 & -0.000\end{array}$

$\begin{array}{llll}0.000 & 0.000 & -0.000\end{array}$

\section{$\begin{array}{llll}0.000 & 0.000 & -0.000\end{array}$}

$\begin{array}{llll}0.000 & 0.000 & -0.000\end{array}$

$0.000 \quad 0.000-0.000$

$\begin{array}{llll}0.000 & 0.000 & -0.000\end{array}$

$\begin{array}{llll}0.000 & 0.000 & -0.000\end{array}$

$\begin{array}{llll}0.000 & 0.000 & -0.000\end{array}$

$\begin{array}{llll}0.000 & 0.000 & -0.000\end{array}$

0.000

$0.000-0.000$

$-0.000$

$0.000-0.000$

$-0.000$

$0.000-0.000$

$-0.000$

$0.000-0.000$ $\begin{array}{llll}0.000 & 0.000 & -0.000\end{array}$

$\begin{array}{llll}0.000 & 0.000 & -0.000\end{array}$

$\begin{array}{cc}\text { POINT } & \text { PLANE } \\ \text { ISOTROPIC } & \text { PEPPENDICULAR } \\ \text { SOURCE } & \text { SOURCE }\end{array}$

SOTROPIC PEPPENDICULAR

$\mathrm{J}(\mathrm{x}) \quad \mathrm{J}(\mathrm{x}) \quad \mathrm{J}(-\mathrm{x})$

$\begin{array}{lll}0.019 & 0.002 & -0.000\end{array}$

$\begin{array}{llll}0.002 & 0.000 & -0.000\end{array}$

$\begin{array}{llll}0.001 & 0.600 & -0.000\end{array}$

$\begin{array}{llll}0.000 & 0.000 & -0.000\end{array}$

$\begin{array}{llll}0.000 & 0.000 & -0.000\end{array}$

$\begin{array}{llll}0.000 & 0.000 & -0.000\end{array}$

$\begin{array}{lll}0.000 & 0.000 & -0.000\end{array}$

$\begin{array}{llll}0.000 & 0.000 & -0.000\end{array}$

$\begin{array}{llll}0.000 & 0.000 & -0.000\end{array}$

$0.000 \quad 0.000-0.000$

$\begin{array}{lll}0.000 & 0.000 & -0.000 \\ 0.000 & 0.000 & -0.000\end{array}$ 
Table 1. Energy Dissipation Function, $J(x)$, and Input Data - Continued

AIR

STOPPING POWER

$$
(d E / d r)_{E_{0}}=9.65 \mathrm{Mev}\left(\mathrm{cm}^{2} / \mathrm{g}\right)
$$

RESIDUAL RANGE

$$
r_{0}=0.00146 \mathrm{~g} / \mathrm{cm}^{2}
$$

\section{OTHER PARAMETERS}

$$
\begin{array}{ll}
a_{1}=1.286 & A_{1}=0.7240 \\
\alpha^{1}=-65.85 & A_{2}=1.164 \\
{ }_{A}{ }^{P T I}=0.9233 & A_{3}=-1.801 \\
{ }_{A} P I P=1.080 & A_{4}=0.9126
\end{array}
$$

SCATTERING COEFFICIENTS

$$
\begin{array}{cc}
\ell & s_{\ell} \\
1 & 1.306 \\
2 & 3.299 \\
3 & 5.766 \\
4 & 8.575 \\
5 & 11.63 \\
6 & 14.87 \\
7 & 18.23 \\
8 & 21.67 \\
9 & 25.17 \\
10 & 28.70 \\
11 & 32.22 \\
12 & 35.72
\end{array}
$$

STOPPING PCWER

$$
(d E / d r)_{E_{O}}=5.77 \mathrm{Mev}\left(\mathrm{cm}^{2} / g\right)
$$

RESIDUAL RANGE

$$
r_{0}=0.00496 \mathrm{~g} / \mathrm{cm}^{2}
$$

OTHER PARATETERS

$$
\begin{array}{ll}
d_{1}=1.329 & A_{1}=0.7547 \\
\alpha=16.71 & A_{2}=0.8725 \\
A^{\text {PTI }}=0.9498 & A_{3}=-1.192 \\
A^{\text {PLP }}=1.114 & A_{4}=0.5644
\end{array}
$$

SCATTERING COEFFICIENTS

$$
\begin{array}{rl}
\ell & \multicolumn{1}{c}{s_{\ell}} \\
1 & 1.254 \\
2 & 3.249 \\
3 & 5.791 \\
4 & 8.757 \\
5 & 12.06 \\
6 & 15.64 \\
7 & 19.43 \\
8 & 23.40 \\
9 & 27.51 \\
10 & 31.72 \\
11 & 36.02 \\
12 & 40.38
\end{array}
$$

$x$
0.000
0.025
0.050
0.075
0.100
0.125
0.150
0.175
0.200
0.225
0.250
0.275
0.300
0.325
0.350
0.375
0.400
0.425
0.450
0.475
SOURC
$E_{0}=0.025 \mathrm{Mev}$

$\begin{array}{cc}\text { POINT } & \text { PLANE } \\ \text { ISOTROPIC } & \text { PERPENDICUI,AR } \\ \text { SOURCE } & \text { SOURCE }\end{array}$

SOURCE

$\begin{array}{lllc}J(x) & J(x) & J(-x) & x \\ 1.000 & 1.53 \varepsilon & 0.538 & 0.500 \\ 1.011 & 1.651 & 0.434 & 0.525 \\ 1.034 & 1.763 & 0.339 & 0.550 \\ 1.069 & 1.876 & 0.257 & 0.575 \\ & & & \\ 1.115 & 1.990 & 0.191 & 0.600 \\ 1.172 & 2.107 & 0.145 & 0.625 \\ 1.239 & 2.227 & 0.122 & 0.650 \\ 1.317 & 2.348 & 0.116 & 0.675 \\ & & & \\ 1.4 n 5 & 2.463 & 0.114 & 0.700 \\ 1.502 & 2.568 & 0.111 & 0.725 \\ 1.609 & 2.662 & 0.106 & 0.750 \\ 1.724 & 2.744 & 0.101 & 0.775 \\ 1.846 & 2.811 & 0.094 & 0.800 \\ 1.976 & 2.862 & 0.085 & 0.825 \\ 2.111 & 2.893 & 0.075 & 0.850 \\ 2.251 & 2.904 & 0.062 & 0.875 \\ 2.394 & 2.890 & 0.048 & 0.900 \\ 2.537 & 2.850 & 0.033 & 0.925 \\ 2.679 & 2.784 & 0.017 & 0.950 \\ 2.815 & 2.691 & 0.004 & 0.975\end{array}$

$E_{0}=0.05 \mathrm{Mev}$

$\begin{array}{cc}\text { POINT } & \text { PLANE } \\ \text { SOTRCPIC } & \text { PERPENDICULAR } \\ \text { SOURCE } & \text { SOURCE }\end{array}$

$\begin{array}{cccccccc}x & J(x) & J(x) & J(-x) & x & J(x) & J(x) & J(-x) \\ 0.000 & 1.000 & 1.497 & 0.497 & 0.500 & 2.878 & 2.557 & -0.009 \\ 0.025 & 1.013 & 1.609 & 0.396 & 0.525 & 3.007 & 2.424 & -0.010 \\ 0.050 & 1.036 & 1.721 & 0.305 & 0.550 & 3.122 & 2.278 & -0.007 \\ 0.075 & 1.070 & 1.834 & 0.227 & 0.575 & 3.217 & 2.121 & -0.002 \\ & & & & & & & \\ 0.100 & 1.114 & 1.950 & 0.165 & 0.600 & 3.279 & 1.953 & 0.002 \\ 0.125 & 1.168 & 2.070 & 0.123 & 0.625 & 3.295 & 1.774 & 0.005 \\ 0.150 & 1.231 & 2.194 & 0.105 & 0.650 & 3.248 & 1.584 & 0.007 \\ 0.175 & 1.303 & 2.320 & 0.100 & 0.675 & 3.117 & 1.384 & 0.008 \\ & & & & & & & \\ 0.200 & 1.385 & 2.433 & 0.099 & 0.700 & 2.884 & 1.174 & 0.007 \\ 0.225 & 1.475 & 2.547 & 0.097 & 0.725 & 2.558 & 0.958 & 0.006 \\ 0.250 & 1.574 & 2.644 & 0.094 & 0.750 & 2.139 & 0.741 & 0.003 \\ 0.275 & 1.681 & 2.728 & 0.089 & 0.775 & 1.691 & 0.533 & 0.001 \\ 0.300 & 1.795 & 2.796 & 0.084 & 0.800 & 1.245 & 0.345 & -0.001 \\ 0.325 & 1.917 & 2.847 & 0.076 & 0.825 & 0.833 & 0.192 & -0.002 \\ 0.350 & 2.045 & 2.878 & 0.066 & 0.850 & 0.483 & 0.083 & -0.002 \\ 0.375 & 2.178 & 2.887 & 0.054 & 0.875 & 0.223 & 0.024 & -0.001 \\ 0.400 & 2.316 & 2.872 & 0.040 & 0.800 & 0.070 & 0.003 & -0.000 \\ 0.425 & 2.457 & 2.831 & 0.026 & 0.925 & 0.011 & 0.000 & -0.000 \\ 0.450 & 2.600 & 2.763 & 0.011 & 0.950 & 0.000 & 0.000 & -0.000 \\ 0.475 & 2.741 & 2.671 & -0.001 & 0.975 & 0.000 & 0.000 & -0.000\end{array}$

POINTT PLANE

ISOTROPIC PERPENDICULAR SOURCE SOURCE

$\mathrm{J}(\mathrm{x}) \quad \mathrm{J}(\mathrm{x}) \quad \mathrm{J}(-\mathrm{x})$

$2.943 \quad 2.57 \ell_{4}-0.006$

$\begin{array}{llll}3.056 & 2.438 & -0.010\end{array}$

$\begin{array}{lll}3.150 & 2.288 & -0.008\end{array}$

$\begin{array}{lll}3.218 & 2.126 & -0.003\end{array}$

$\begin{array}{lll}3.252 & 1.956 & 0.001\end{array}$

$\begin{array}{lll}3.243 & 1.776 & 0.005\end{array}$

$\begin{array}{lll}3.182 & 1.588 & 0.007\end{array}$

$\begin{array}{lll}3.060 & 1.389 & 0.008\end{array}$

$\begin{array}{lll}2.867 & 1.183 & 0.008\end{array}$

$\begin{array}{lll}2.597 & 0.971 & 0.006\end{array}$

$\begin{array}{lll}2.248 & 0.758 & 0.004\end{array}$

$\begin{array}{lll}1.827 & 0.552 & 0.001\end{array}$

$\begin{array}{llll}1.356 & 0.364 & -0.001\end{array}$

$\begin{array}{llll}0.879 & 0.206 & -0.002\end{array}$

$\begin{array}{llll}0.461 & 0.093 & -0.003\end{array}$

$\begin{array}{llll}0.170 & 0.028 & -0.002\end{array}$

$\begin{array}{llll}0.033 & 0.004 & -0.001\end{array}$

$\begin{array}{lll}0.002 & 0.000 & 0.000\end{array}$

$\begin{array}{lll}0.000 & 0.000 & 0.000\end{array}$

$0.000 \quad 0.000 \quad 0.000$
POINT YLANE

ISOTROPIC PERPENDICULAR SOURCE SOURCE

$\mathrm{J}(\mathrm{x}) \quad \mathrm{J}(\mathrm{x}) \quad \mathrm{J}(-\mathrm{x})$ 
STOPPING PONER

$$
(\mathrm{dE} / \mathrm{dr})_{E_{0}}=3.60 \mathrm{Mev}\left(\mathrm{cm}^{2} / \mathrm{g}\right)
$$

RESIDUAL RANGE

$$
r_{0}=0.0164 \mathrm{~g} / \mathrm{cm}^{2}
$$

OTHER PARANETERS

$$
\begin{array}{ll}
d_{1}=1.2670 & A_{1}=0.6992 \\
\alpha=29.16 & A_{2}=0.9039 \\
A^{\text {PTI }}=0.9328 & A_{3}=-1.0148 \\
A^{\text {PLP }}=1.101 & A_{4}=0.41452
\end{array}
$$

SCATTERING COEFFICIENTS

$\begin{array}{cc}l & s_{l} \\ 1 & 1.225 \\ 2 & 3.247 \\ 3 & 5.882 \\ 4 & 9.019 \\ 5 & 12.58 \\ 6 & 16.49 \\ 7 & 20.70 \\ 8 & 25.18 \\ 9 & 29.89 \\ 10 & 34.79 \\ 11 & 39.87 \\ 12 & 45.08\end{array}$

STOPPING POWER

$$
(\mathrm{dE} / \mathrm{dr})_{E_{0}}=2.45 \mathrm{Mev}\left(\mathrm{cm}^{2} / \mathrm{g}\right)
$$

RESIDUAL RANGE

$$
r_{0}=0.0513 \mathrm{~g} / \mathrm{cm}^{2}
$$

OTHER PARAMETERS

$$
\begin{array}{ll}
a_{1}=1.246 & A_{1}=0.6401 \\
\alpha=18.84 & A_{2}=0.9019 \\
A^{P T I}=0.928 & A_{3}=-1.004 \\
A^{P L P}=0.1100 & A_{4}=0.4624
\end{array}
$$

SCATTERING COEFICIENTS

$$
\begin{array}{cc}
l & s_{\ell} \\
1 & 1.183 \\
2 & 3.206 \\
3 & 5.894 \\
4 & 9.143 \\
5 & 12.88 \\
6 & 17.04 \\
7 & 21.59 \\
8 & 26.48 \\
9 & 31.67 \\
10 & 37.14 \\
11 & 42.90 \\
12 & 48.89
\end{array}
$$

AIR

$E_{0}=0.10 \mathrm{Mev}$

$\begin{array}{cc}\text { POIIT } & \text { PLANE } \\ \text { ISOTROPIC } & \text { PERPENDICULAR } \\ \text { SOURCE } & \text { SCURCE }\end{array}$

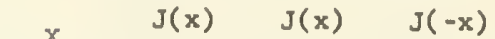

0.000

0.025

0.050

0.075

0.100

0.125

0.150

0.175

0.200

0.225

0.250

0.275

0.300

0.325

0.350

0.375

0.400

0.425

0.450

0.475

$$
E_{0}=0.20 \mathrm{Mev}
$$

$\begin{array}{cc}\text { POINT } & \text { PLANE } \\ \text { ISOTROPIC } & \text { PERPENDICULAR } \\ \text { SOURCE } & \text { SOURCE }\end{array}$

$x$

0.000

0.025

0.050

0.075

0.100

0.125

0.150

0.175

0.200

0.225

0.250

0.275

0.300

0.325

0.350

0.375

0.400

0.425

0.450

0.475

\section{$J(x)$}

1.000

1.009

1.027

1.052

1.085

1.126

1.174

1.230

1.293

1.363

1.440

1.525

1.616

1.714

1.818

1.929

$$
\mathrm{J}(\mathrm{x})
$$

1.395

1.493

1.592

0.395

0.306

0.225

1.693

0.156

1.797

1.906

2.022

0.103

0.069

0.056

2.140

2.251

2.352

2.442

2.518

0.063

0.067

0.068

0.068

2.579

2.623

0.065

0.060

2.647

0.051

0.040

2.045

2.167

2.292

2.421
$\mathrm{J}(-\mathrm{x})$
2.628

2.583

0.028

0.014

2.514

2.425
0.000

$-0.009$ $x$

0.500

0.525

0.550

0.575

0.600

0.625

0.650

0.675

0.700

0.725

0.750

0.775

0.800

0.825

0.850

0.875

0.900

0.925

0.950

0.975
POINT

ISOTROPIC PERPENDICULAR SCURCE SOURCE

$$
\mathrm{J}(\mathrm{x})
$$

$\mathrm{J}(\mathrm{x})$

$\mathrm{J}(-\mathrm{x})$

2.749

2.876

2.993

3.090

2.472

2.344

2.205

$\begin{array}{ll}2.205 & -0.007 \\ 2.055 & -0.002\end{array}$

$\begin{array}{lll}3.158 & 1.895 & 0.002\end{array}$

$3.181 \quad 1.725$

$3.138 \quad 1.543$

0.005

3.008

1.351

0.006

2.780

2.466

1.149

0.940

0.730

2.098

0.527

0.006

0.005

1.695

1.275

0.863

0.496

$0.343-0.001$

0.218

0.191

0.083

0.024

0.060

0.006

$0.003-0.000$

0.000

$0.000-0.000$

0.000

$\begin{array}{ll}0.000 & -0.000 \\ 0.000 & -0.000\end{array}$
POINT PLANE

ISOTROPIC PERPENDICULAR SOURCE

$\mathrm{J}(\mathrm{x})$

$\mathrm{J}(\mathrm{x})$

SOURCE

0.500

0.525

0.550

0.575

2.551

2.677

2.794

2.892

2.319

2.200

2.071

1.932

$J(-x)$

0.600

2.959

1.783

2.975

1.624

0.650

0.675

2.921

1.454

1.273

2.780

1.083

2.578

0.725

0.750

2.311

0.886

0.688

0.496

0.006

0.775

1.626

0.800

1.228

0.322

0.179

0.078

0.850

0.466

0.195

$\begin{array}{lll}0.022 & -0.001\end{array}$

0.900

0.925

0.048

0.004

0.950

0.975

0.000

$0.003-0.000$

$0.000-0.000$

0.000

$\begin{array}{ll}0.000 & -0.000 \\ 0.000 & -0.000\end{array}$ 
Table 1. Energy Dissipation Function, $J(x)$, and Input Data - Continued

AIR

$E_{0}=0.40 \mathrm{MeV}$

STOPFING PC'NER

$(\mathrm{dE} / \mathrm{dr})_{E_{0}}=1.89 \mathrm{~N} \vee v\left(\mathrm{~cm}^{2} / \varepsilon\right)$

RESIDUAL RANGE

$r_{O}=0.147 \mathrm{~g} / \mathrm{cm}^{2}$

OTHER PARAMETERS

$d_{1}=1.227 \quad A_{1}=0.5361$

$a=9.408 \quad A_{2}=0.9248$

${ }_{A} \mathrm{PTI}=0.9134 \quad A_{3}=-0.7547$

$A^{\text {PLF }}=1.084 \quad A_{4}=0.2938$

SCATTERING COEFFICIENTS

$\begin{array}{cc}l & s_{l} \\ 1 & 1.109 \\ 2 & 3.067 \\ 3 & 5.712 \\ 4 & 8.952 \\ 5 & 12.72 \\ 6 & 16.97 \\ 7 & 21.65 \\ 8 & 26.73 \\ 9 & 32.17 \\ 10 & 37.96 \\ 11 & 44.06 \\ 12 & 50.46\end{array}$

STOPPING POWER

$(\mathrm{dE} / \mathrm{dr})_{E_{O}}=1.70 \mathrm{Mev}\left(\mathrm{cm}^{2} / \mathrm{g}\right)$

RESIDOAL RANGE

$r_{0}=0.317 \mathrm{~g} / \mathrm{cm}^{2}$

CTHER PARAMETERS

$d_{1}=1.215 \quad A_{1}=0.4343$

${ }_{\mathrm{PTI}}=4.830 \quad \mathrm{~A}_{2}=0.9874$

$\begin{array}{ll}A_{A} \text { PLP }=0.9157 & A_{3}=-0.7402 \\ A_{4}=0.3184 & A_{4}=0.30\end{array}$

SCATTERING COERTICIENTS

\begin{tabular}{cc}
$l$ & \multicolumn{1}{c}{$s$} \\
1 & 1.007 \\
2 & 2.822 \\
3 & 5.302 \\
4 & 8.368 \\
5 & 11.96 \\
6 & 16.04 \\
7 & 20.57 \\
8 & 25.51 \\
9 & 30.84 \\
10 & 36.52 \\
11 & 42.59 \\
12 & 48.96
\end{tabular}

$\begin{array}{lc}\text { POINT } & \text { PLANE } \\ \text { SOTROPIC } & \text { FERPENDICUTAAR } \\ \text { SOURCE } & \text { SOURCE }\end{array}$

SOURCE SOURCE

$\begin{array}{cccc}x & J(x) & J(x) & J(-x) \\ 0.000 & 1.000 & 1.314 & 0.314 \\ 0.025 & 1.008 & 1.400 & 0.237 \\ 0.050 & 1.022 & 1.486 & 0.168 \\ 0.075 & 1.043 & 1.574 & 0.109 \\ 0.100 & 1.070 & 1.666 & 0.064 \\ 0.125 & 1.102 & 1.763 & 0.035 \\ 0.150 & 1.141 & 1.867 & 0.027 \\ 0.175 & 1.186 & 1.974 & 0.033 \\ & & & \\ 0.200 & 1.237 & 2.074 & 0.041 \\ 0.225 & 1.293 & 2.165 & 0.047 \\ 0.250 & 1.355 & 2.245 & 0.051 \\ 0.275 & 1.423 & 2.311 & 0.053 \\ 0.300 & 1.497 & 2.364 & 0.052 \\ 0.325 & 1.576 & 2.399 & 0.048 \\ 0.350 & 1.660 & 2.415 & 0.041 \\ 0.375 & 1.749 & 2.411 & 0.032 \\ 0.400 & 1.843 & 2.385 & 0.020 \\ 0.425 & 1.942 & 2.336 & 0.007 \\ 0.450 & 2.044 & 2.266 & -0.005 \\ 0.475 & 2.149 & 2.179 & -0.012\end{array}$

$E_{0}=0.70 \mathrm{Mev}$

$\begin{array}{cc}\text { POINT } & \text { PLANE } \\ \text { ISOTROPIC } & \text { PERPENDICULAR } \\ \text { SOURCE } & \text { SOURCE }\end{array}$

$\mathrm{J}(\mathrm{x}) \quad \mathrm{J}(\mathrm{x}) \quad \mathrm{J}(-\mathrm{x})$

$x$

0.000

0.025

0.050

0.075

0.100

0.125

0.150

0.175

0.200

0.225

0.250

0.275

0.300

0.325

0.350

0.375

0.400

0.425

0.450

0.475 $\begin{array}{lll}1.000 & 1.271 & 0.271\end{array}$

$\begin{array}{lll}1.013 & 1.423 & 0.136\end{array}$

$1.028 \quad 1.501$

0.083

$1.049 \quad 1.581$

$1.075 \quad 1.667$

0.042

$1.10 \mathrm{~h}$

1.758

0.018

0.012

1.143

1.851

0.020

1.185

1.231

1.937

2. 014

0.030

1.283

2.080

2.134

0.038

0.043

1.400

2.172

1.466

1.535

2.195

2.198

2.182

0.046

0.042

0.036

0.026

1.686

1.766

2.145

0.014

1.848

2.088

0.002

1.931 $\begin{array}{ll}2.013 & -0.008 \\ 1.925 & -0.013\end{array}$ $\begin{array}{lll}1.004 & 1.347 & 0.200\end{array}$ $x$
0.500

0.525

0.550

0.575

0.600

0.625

0.650

0.675

0.700

0.725

0.750

0.775

0.800

0.825

0.850

0.875

0.900

0.925

0.950

0.975
POINT

ISOTROPIC PERPENDICULAR SOURCE SOURCE

$\mathrm{J}(\mathrm{x}) \mathrm{J}(\mathrm{x}) \quad \mathrm{J}(-\mathrm{x})$

2.255

2.358

2.4 .55

0.537

2.592

2.606

2.560

2.443

2.262

2.030

1.752

1.434

1.088

0.737

0.418

0.177

0.044

0.004

0.000

0.000
$2.079-0.013$

$1.969-0.010$

$1.850-0.006$

$1.724-0.002$

$\begin{array}{lll}1.591 & 0.001\end{array}$

1.448

1.297

1.136

0.003

0.005

$0.967 \quad 0.005$

0.792

0.616

0.445

0.004

0.002

$0.290-0.001$

$0.161-0.002$

c. $070-0.002$

$0.020-0.001$

$0.003-0.000$

$0.000-0.000$

$0.000 \quad 0.000$

$0.000 \quad c .000$ $\begin{array}{cc}\text { POINT } & \text { PLANE } \\ \text { ISOTRCPIC } & \text { PERFENDICULAR } \\ \text { SOURCE } & \text { SOURCE }\end{array}$

$x$
0.500
0.525
0.550
0.575

$J(x)$

2.013

$\mathrm{J}(\mathrm{x})$

$\mathrm{J}(-\mathrm{x})$

2.092

1.827

$-0.012$

2.164

$1.722-0.009$

2.224

1.612

1.496

$-0.005$

0.600

2.26

0.625

0.650

0.675

2. 267

1.374

1.246

2.2241 .110

$2.118 \quad 0.968$

0.001

0.700

0.725

1.948

0.819

0.667

1.477

1.198

0.516

0.370

0.004

0.775

0.800

0.825

0.850

0.875

0.902

0.239

.609

0.346

0.132

0.148

$0.016-0.001$

0.900

0.925

0.950

0.975
0.038

0.003

0.000

0.000
$0.002-0.000$

$0.000-0.000$

$0.000-0.000$

$0.000-0.000$ 
Table 1. Energy Dissipation Function, $J(x)$, and Input Data - Continued

$$
E_{0}=1.00 \mathrm{MeV}
$$

STOPPING POWER

$$
(\mathrm{dE} / \mathrm{dr})_{\mathrm{E}_{\mathrm{O}}}=1.65 \mathrm{Nev}\left(\mathrm{cm}^{2} / \mathrm{g}\right)
$$

RESIDUAL RANGE

$$
r_{0}=0.497 \mathrm{~g} / \mathrm{cm}^{2}
$$

OTHER PARAMETERS

$$
\begin{array}{ll}
d_{1}=1.223 & A_{1}=0.3807 \\
a \quad=2.968 & A_{2}=1.205 \\
A^{P T I}=0.8594 & A_{3}=-1.095 \\
A^{P L P}=0.1022 & A_{4}=0.5094
\end{array}
$$

SCATTERING COEFFICIENTS

\begin{tabular}{cc}
$\ell$ & \multicolumn{1}{c}{$s_{\ell}$} \\
1 & 0.9151 \\
2 & 2.522 \\
3 & 4.874 \\
4 & 7.721 \\
5 & 11.08 \\
6 & 14.90 \\
7 & 19.15 \\
8 & 23.82 \\
9 & 28.87 \\
10 & 34.29 \\
11 & 40.05 \\
12 & 46.14
\end{tabular}

STOPPING PONER

$$
(d E / d r)_{E_{O}}=1.68 \mathrm{Mev}\left(\mathrm{cm}^{2} / \mathrm{g}\right)
$$

RESIDUAL RANGE

$$
r_{0}=1.10 \mathrm{~g} / \mathrm{cm}^{2}
$$

OTHER PARAYETERS

$$
\begin{array}{ll}
\mathrm{d}_{1}=1.251 & \mathrm{~A}_{1}=0.2699 \\
\alpha=1.230 & A_{2}=1.856 \\
A^{\mathrm{PTI}}=0.7775 & A_{3}=-2.638 \\
A^{\mathrm{PT} P}=0.9236 & A_{4}=1.512
\end{array}
$$

SCATTERING COEFFICIENTS

\begin{tabular}{cc}
$l$ & \multicolumn{1}{c}{$s$} \\
1 & 0.6902 \\
2 & 1.966 \\
3 & 3.738 \\
4 & 5.955 \\
5 & 8.592 \\
6 & 11.62 \\
7 & 15.01 \\
8 & 18.75 \\
9 & 22.83 \\
10 & 27.22 \\
11 & 31.23 \\
12 & 36.91
\end{tabular}
SOURCE

$x$
0.000
0.025
0.050
0.075
0.100
0.125
0.150
0.175
0.200
0.225
0.250
0.275
0.300
0.325
0.350
0.375
0.400
0.425
0.450
0.475

$$
\mathrm{J}(\mathrm{x})
$$

$$
\mathrm{J}(\mathrm{x})
$$

1.000

1.186

1.001

1.007

1.239

1.017

1.294

1.352

$1.032 \quad 1.416$

1.051

1.416
1.487

1.073

1.567

1.100

1.654

1.132

1.737

1.167

1.737
1.81

1.206

1.882

1.250

1.942

$1.298 \quad 1.990$

1.349

1.405

2.025
2.046

1.465

2.049

\section{529}

2.035

1.597

2.000

1.669

1.946

$E_{0}=2.00 \mathrm{Mev}$

POINT PLANE ISOTROPIC PERPENDICULAR SOURCE

$\mathrm{J}(-\mathrm{x})$

0.186

0.135

0.090

0.050

0.020

0.002

$-0.003$

$-0.003$

0.011

0.018

0.024

0.028

0.030

0.030

0.028
0.023

0.016

0.007

$-0.003$

POIIT

ISOTROPIC SOURCE

PLANE $\mathrm{J}(\mathrm{x})$

$x$
0.000
0.025
0.050
0.075
0.100
0.125
0.150
0.175

1.000

0.993

0.989

0.988

0.990

0.99

1.003

1.014

0.200

0.225

0.250

0.275

0.300

0.325

0.350

0.375

0.400

0.425

0.450

0.475 $\begin{array}{lll}1.028 & 1.423 & -0.001\end{array}$

$\begin{array}{lll}1.045 & 1.485 & 0.003\end{array}$

$\begin{array}{lll}1.066 & 1.543 & 0.007\end{array}$

1.089

1.597

0.009

1.116

1.447

1.645
1.687

1.182

1. 221

1.721

1.746

0.012

0.013

0.014

1.264

1.760

1.313

1.762

1.368

1.428

0.013
SOURCE

$x^{x}$
0.500
0.525
0.550
0.575

0.600

0.625

0.650

0.675

0.700

0.725

0.750

0.775

0.800

0.825

0.850

0.875

0.900

0.925

0.950

0.975 
Table 1. Energy Dissipation Function, $J(x)$, and Input Data - Continued

AIR

$E_{0}=4.00 \mathrm{Mev}$

STOPPING POWER

$$
(\mathrm{dE} / \mathrm{dr})_{\mathrm{E}_{\mathrm{o}}}=1.78 \mathrm{Mev}\left(\mathrm{cm}^{2} / \mathrm{g}\right)
$$

RESIDUAL RANGE

$$
r_{0}=2.26 \mathrm{~g} / \mathrm{cm}^{2}
$$

OTHER PARAIETERS

$$
\begin{array}{ll}
\mathrm{d}_{1}=1.326 & \mathrm{~A}_{1}=0.2624 \\
a=0.5283 & A_{2}=1.034 \\
{ }_{\mathrm{ATI}}=0.6663 & \mathrm{~A}_{3}=-0.6649 \\
{ }_{\mathrm{A} P P}=0.7867 & \mathrm{~A}_{4}=0.3688
\end{array}
$$

POINT ISOTROPIC PERPENDICULAR SOURCE SOURCE

$\begin{array}{ccccc}x & J(x) & J(x) & J(-x) & x \\ 0.000 & 1.000 & 1.038 & 0.038 & 0.500 \\ 0.025 & 0.998 & 1.046 & 0.028 & 0.525 \\ 0.050 & 0.997 & 1.056 & 0.019 & 0.550 \\ 0.075 & 0.998 & 1.069 & 0.011 & 0.575 \\ & & & & \\ 0.100 & 1.000 & 1.087 & 0.004 & 0.600 \\ 0.125 & 1.004 & 1.110 & -0.000 & 0.625 \\ 0.150 & 1.009 & 1.142 & -0.003 & 0.650 \\ 0.175 & 1.016 & 1.184 & -0.003 & 0.675 \\ 0.200 & 1.024 & 1.230 & -0.002 & 0.700 \\ 0.225 & 1.033 & 1.274 & -0.000 & 0.725 \\ 0.250 & 1.044 & 1.317 & 0.001 & 0.750 \\ 0.275 & 1.057 & 1.359 & 0.002 & 0.775 \\ & & & & \\ 0.300 & 1.071 & 1.398 & 0.003 & 0.800 \\ 0.325 & 1.087 & 1.435 & 0.003 & 0.825 \\ 0.350 & 1.105 & 1.468 & 0.004 & 0.850 \\ 0.375 & 1.125 & 1.497 & 0.004 & 0.875 \\ 0.400 & 1.147 & 1.520 & 0.004 & 0.900 \\ 0.425 & 1.171 & 1.537 & 0.004 & 0.925 \\ 0.450 & 1.197 & 1.544 & 0.003 & 0.950 \\ 0.475 & 1.226 & 1.541 & 0.002 & 0.975\end{array}$
$E_{0}=10.00 \mathrm{Mev}$

STOPPING PONER

$$
(\mathrm{dE} / \mathrm{dr})_{\mathrm{E}_{\mathrm{O}}}=1.97 \mathrm{Mev}\left(\mathrm{cm}^{2} / \mathrm{g}\right)
$$

RESIDUAL RANGE

$$
r_{O}=5.44 \mathrm{~g} / \mathrm{cm}^{2}
$$

OTHER PARAMETERS

$$
\begin{array}{ll}
d_{1}=1.682 & A_{1}=0.1200 \\
\alpha=0.1557 & A_{2}=1.942 \\
{ }_{A} \text { PTI }=0.5002 & A_{3}=-2.596 \\
A^{P L P}=0.5760 & A_{4}=1.534
\end{array}
$$

SCATTERING COEFFICIENTS

$$
\begin{array}{rl}
l & s_{l} \\
1 & 0.2266 \\
2 & 0.6534 \\
3 & 1.257 \\
4 & 2.026 \\
5 & 2.951 \\
6 & 4.024 \\
7 & 5.240 \\
8 & 6.594 \\
9 & 8.082 \\
10 & 9.700 \\
11 & 11.45 \\
12 & 13.32
\end{array}
$$

$\begin{array}{cc}\text { POINT } & \text { PLANE } \\ \text { ISOTROPIC } & \text { PERPENDICULAR } \\ \text { SOURCE } & \text { SOURCE }\end{array}$

$x$

0.000

0.025

0.050

0.075

0.100

0.125

0.150

0.175

0.200

0.225

0.250

0.275

$J(x)$

$$
\mathrm{J}(\mathrm{x})
$$

$J(-x)$

1.000

1.010

0.010

0.997

0.995

1.008

1.009

0.009

0.994

1.011

0.008

0.008

$0.993 \quad 1.014$

0.9921 .019

0.993

0.993

1.027

0.007

0.006

0.006

1.036

0.005

$0.996 \quad 1.060$

$0.999 \quad 1.075$

1.001

1.092

0.003

0.003

1.005

0.300

0.325

1.008

1.11

1.012

1.132

0.350

0.375

1.016

$1.155-0.001$

0.400

0.425

0.450

1.020

$1.181-0.002$

1.026

$1.207-0.003$

$1.234-0.004$

1.033

$\begin{array}{ll}1.260 & -0.005\end{array}$

$\begin{array}{lllll}0.475 & 1.041 & 1.284 & -0.006 & 0.975\end{array}$ $\begin{array}{lll}0.994 & 1.047 & 0.004\end{array}$ $\mathrm{x}$

0.500

0.550

0.575

0.600

0.625

0.650

0.675

0.700

0.725

0.750

0.775

0.800

0.825

0.850

0.875

0.900

0.925

0.950
0.525
POINT PLANE

ISOTROPIC PERPENDICULAR SOURCE SOURCE

$\mathrm{J}(\mathrm{x}) \mathrm{J}(\mathrm{x}) \quad \mathrm{J}(-\mathrm{x})$

$\begin{array}{lll}1.257 & 1.526 & 0.001\end{array}$

$\begin{array}{lll}1.291 & 1.496 & -0.001\end{array}$

$\begin{array}{lll}1.328 & 1.450 & -0.003\end{array}$

$\begin{array}{lll}1.367 & 1.388 & -0.004\end{array}$

$\begin{array}{lll}1.408 & 1.312 & -0.004\end{array}$

$1.447 \quad 1.224 \quad-0.003$

$\begin{array}{llll}1.479 & 1.129 & -0.001\end{array}$

$\begin{array}{lll}1.496 & 1.027 & 0.001\end{array}$

$\begin{array}{lll}1.486 & 0.916 & 0.002\end{array}$

$\begin{array}{lll}1.438 & 0.797 & 0.002\end{array}$

$\begin{array}{lll}1.343 & 0.659 & 0.002\end{array}$

$\begin{array}{lll}1.191 & 0.543 & 0.002\end{array}$

$\begin{array}{lll}0.978 & 0.395 & 0.001\end{array}$

$\begin{array}{llll}0.716 & 0.261 & -0.000\end{array}$

$\begin{array}{llll}0.437 & 0.143 & -0.001\end{array}$

$\begin{array}{llll}0.198 & 0.057 & -0.001\end{array}$

$\begin{array}{lll}0.055 & 0.013-0.000\end{array}$

$\begin{array}{llll}0.008 & 0.001 & -0.000\end{array}$

$\begin{array}{llll}0.003 & 0.000 & -0.000\end{array}$

$\begin{array}{llll}0.005 & 0.000 & -0.000\end{array}$
POINT PLANE

ISOTROFIC PERPENDICULAR SOURCE SOURCE

$\mathrm{J}(\mathrm{x}) \mathrm{J}(\mathrm{x}) \quad \mathrm{J}(-\mathrm{x})$

$\begin{array}{llll}1.051 & 1.303 & -0.007\end{array}$

$\begin{array}{llll}1.063 & 1.314 & -0.007\end{array}$

$\begin{array}{llll}1.079 & 1.314 & -0.007\end{array}$

$\begin{array}{llll}1.099 & 1.301 & -0.004\end{array}$

$\begin{array}{lll}1.123 & 1.279 & 0.001\end{array}$

$\begin{array}{lll}1.154 & 1.252 & 0.007\end{array}$

$\begin{array}{lll}1.193 & 1.220 & 0.012\end{array}$

$\begin{array}{lll}1.238 & 1.179 & 0.015\end{array}$

$\begin{array}{lll}1.285 & 1.127 & 0.016\end{array}$

$\begin{array}{lll}1.322 & 1.059 & 0.014\end{array}$

$\begin{array}{lll}1.330 & 0.968 & 0.007\end{array}$

$\begin{array}{llll}1.290 & 0.849 & -0.004\end{array}$

$\begin{array}{llll}1.184 & 0.695 & -0.018\end{array}$

$\begin{array}{llll}0.996 & 0.511 & -0.028\end{array}$

$\begin{array}{llll}0.728 & 0.315 & -0.023\end{array}$

$\begin{array}{llll}0.420 & 0.142 & -0.000\end{array}$

$\begin{array}{lll}0.162 & 0.029 & 0.020\end{array}$

$\begin{array}{llll}0.031 & -0.009 & -0.017\end{array}$

$\begin{array}{llll}0.001 & -0.004 & 0.005\end{array}$

$0.000 \quad-0.000 \quad 0.000$ 
Table 1. Energy Disipation Function, $J(x)$, and Input Data - Continued

POLYSTYRENE

$$
E_{0}=0.025 \mathrm{Mev}
$$

STOPPING PCWER

$$
(d E / d r)_{E_{0}}=11.2 \mathrm{Mev}\left(\mathrm{cm}^{2} / \mathrm{g}\right)
$$

RESIDUAL RANGE

$$
r_{0}=0.00125 \mathrm{~g} / \mathrm{cm}^{2}
$$

OTHER PARAMETERS

$$
\begin{array}{ll}
d_{1}=0.9338 & A_{1}=0.7245 \\
a=-63.71 & A_{2}=1.169 \\
A^{\text {FTI }}=0.6711 & A_{3}=-1.781 \\
A^{\text {PLP }}=0.7937 & A_{4}=0.8868
\end{array}
$$

SCATTERING COERYICIENTS

\begin{tabular}{rl}
$l$ & \multicolumn{1}{c}{$s_{l}$} \\
1 & 0.9487 \\
2 & 1.457 \\
3 & 4.251 \\
4 & 6.360 \\
5 & 8.733 \\
6 & 11.28 \\
7 & 13.94 \\
8 & 16.71 \\
9 & 19.91 \\
10 & 23.19 \\
11 & 26.55 \\
12 & 29.98
\end{tabular}

STORPING POWER

$$
(d E / d r)_{E_{0}}=6.64 \operatorname{Mev}\left(\mathrm{cm}^{2} / g\right)
$$

RESIDITAL RANGE

$$
r_{0}=0.00428 \mathrm{~g} / \mathrm{cm}^{2}
$$

OTHER PARAMETERS

$$
\begin{array}{ll}
d_{1}=0.9697 & A_{1}=0.7637 \\
a=13.56 & A_{2}=0.9428 \\
A^{P T I}=0.6898 & A_{3}=-1.383 \\
A^{P L P}=0.8173 & A_{4}=0.6763
\end{array}
$$

SCATTERING COEPTICIENTS

$$
\begin{array}{cc}
l & \multicolumn{1}{c}{s l} \\
1 & 0.9031 \\
2 & 2.349 \\
3 & 4.202 \\
4 & 6.375 \\
5 & 8.807 \\
6 & 11.45 \\
7 & 14.27 \\
8 & 17.23 \\
9 & 20.31 \\
10 & 23.48 \\
11 & 26.72 \\
12 & 30.02
\end{array}
$$

$\begin{array}{cc}\text { POINT } & \text { PLANE } \\ \text { ISOTRCPIC } & \text { PERPENDICULAR } \\ \text { SOURCE } & \text { SOURCE }\end{array}$

$\begin{array}{clll}x & J(x) & J(x) & J(-x) \\ 0.000 & 1.000 & 1.342 & 0.342 \\ 0.025 & 1.010 & 1.414 & 0.278 \\ 0.050 & 1.027 & 1.488 & 0.218 \\ 0.075 & 1.052 & 1.565 & 0.163 \\ & & & \\ 0.100 & 1.084 & 1.647 & 0.116 \\ 0.125 & 1.123 & 1.735 & 0.079 \\ 0.150 & 1.169 & 1.833 & 0.058 \\ 0.175 & 1.221 & 1.942 & 0.053\end{array}$

0.200

0.225

0.250

0.275

0.300

0.325

0.350

0.375

0.400

0.425

0.450

0.475

\subsection{0}

\subsection{6}

1.419

1.499

2.055

2.164

2.270

2.370

1.585

1.679

2.465

1.779

2.553

2.631

2.698

1.999

2.118

2.242

2.750

2.786

2.800

2.370
0.055

0.057

0.058
0.059

0.058

0.057

0.055

0.051

0.04 ?

0.037

0.028
$E_{0}=0.05 \mathrm{Mev}$

POINT PLANE

ISCTRCPIC PERPENDICULAR

SUITRCE

SOURCE

$x$

$J(x)$

$J(x)$

$\mathrm{J}(-\mathrm{x})$

0.000

1.000

1.300

0.300

0.050

0.075

1.010

1.369

1.026

1.440

0.240

0.186

0.137

0.100

0.125

0.150

1.079

1.114

1.599

1.156

1.693

0.175

1.205

1.801

0.097

0.070

0.059

0.057

0.200

1.259

0.225

1.321

2.029

0.056

1.389

2.139

0.275

1.464

2.245

0.054

0.052

2.346

0.050

1.545

2.442

0.048

0.325

0.350

1.634

2.530

1.729

2.609

0.045

0.042

1.832

2.677

0.400

0.425

0.450

0.475
1.941
2.058
2.181

2.311
2.732

2.770

2.788

2.784
0.033

0.027

0.021

0.013 $x$

0.525

0.550

0.575

0.600

0.625

0.650

0.675

0.700

0.725

0.750

0.775

0.800

0.825

0.850

0.875

0.900

0.925

0.950

0.975
POINT ISOTROPIC PERPENDICUIAAR SOIIRCE

$$
J(x)
$$

2.448

2.587

2.729

2.867

2.996

3.107

3.191

3.237

3.231

3.156

2.993

2.724

2.334

1.826

1.238

0.657

0.221

0.028

0.000

0.000
PLANE

PERPENDICULAR SOURCE

$\mathrm{J}(\mathrm{x}) \quad \mathrm{J}(-\mathrm{x})$

$2.754 \quad 0.003$

$2.690-0.008$

$2.601-0.016$

$2.491-0.016$

$2.367 \quad-0.011$

$2.231-0.004$

2.0820 .002

1.9190 .006

$\begin{array}{ll}1.739 & 0.009\end{array}$

$\begin{array}{lll}1.540 & 0.010\end{array}$

$\begin{array}{ll}1.321 & 0.010\end{array}$

$\begin{array}{ll}1.083 & 0.007\end{array}$

$\begin{array}{lll}0.831 & 0.003\end{array}$

$\begin{array}{lll}0.576 & -0.001\end{array}$

$\begin{array}{lll}0.341 & -0.004\end{array}$

$0.154-0.005$

$\begin{array}{lll}0.042 & -0.003\end{array}$

$\begin{array}{lll}0.004 & -0.001\end{array}$

$0.000-0.000$

$0.000 \quad 0.000$ 
Table 1. Energy Dissipation Function, $J(x)$, and Input Data - Continued

POLYSTYRENE

$E_{0}=0.10 \mathrm{Mev}$

STOPFING POWER

$$
(d E / d r)_{E_{0}}=4.13 \operatorname{Mov}\left(\mathrm{cm}^{2} / \mathrm{g}\right)
$$

RESIDUAL RANGE

$$
r_{0}=0.0142 \mathrm{~g} / \mathrm{cm}^{2}
$$

OTHER PARAMETERS

$$
\begin{array}{ll}
d_{1}=0.9090 & A_{1}=0.7102 \\
A=45.20 & A_{2}=0.8488 \\
A^{P T I}=0.6738 & A_{3}=-0.9880 \\
A^{\text {PLP }}=0.8051 & A_{4}=0.4290
\end{array}
$$

SCATTERING COEFFICIENTS

$\begin{array}{cc}l & s l \\ 1 & 0.8894 \\ 2 & 2.367 \\ 3 & 4.303 \\ 4 & 6.618 \\ 5 & 9.254 \\ 6 & 12.17 \\ 7 & 15.32 \\ 8 & 18.68 \\ 9 & 22.23 \\ 10 & 25.93 \\ 11 & 29.78 \\ 12 & 33.75\end{array}$

STOPPING POWER

$$
(d E / d r)_{E_{O}}=2.79 \mathrm{Mov}\left(\mathrm{cm}^{2} / g\right)
$$

RESIDUAL RANGE

$$
r_{0}=0.0448 \mathrm{p} / \mathrm{cm}^{2}
$$

OTHER PARAMETERS

$$
\begin{array}{ll}
a_{1}=0.9034 & A_{1}=0.6469 \\
a^{\text {PTI }}=20.18 & A_{2}=0.8812 \\
A^{\text {PTI }}=0.6770 & A_{3}=-0.9830 \\
A^{\text {PLP }}=0.8119 & A_{4}=0.4548
\end{array}
$$

SCATTERING COEPFICIENTS

\begin{tabular}{rl}
$l$ & \multicolumn{1}{c}{$s_{l}$} \\
1 & 0.8607 \\
2 & 2.340 \\
3 & 4.314 \\
4 & 6.709 \\
5 & 9.472 \\
6 & 12.56 \\
7 & 15.94 \\
8 & 19.59 \\
9 & 23.47 \\
10 & 27.57 \\
11 & 31.87 \\
12 & 36.35
\end{tabular}

$\begin{array}{cc}\text { POINT } & \text { PLANE } \\ \text { ISOTROPIC } & \text { PERPENDICULAR } \\ \text { SOURCE } & \text { SOURCE }\end{array}$

$\begin{array}{ccccc}x & J(x) & J(x) & J(-x) & x \\ 0.000 & 1.000 & 1.252 & 0.252 & 0.500 \\ 0.025 & 1.011 & 1.319 & 0.196 & 0.525 \\ 0.050 & 1.028 & 1.391 & 0.146 & 0.550 \\ 0.075 & 1.052 & 1.469 & 0.105 & 0.575 \\ 0.100 & 1.082 & 1.561 & 0.078 & 0.600 \\ 0.125 & 1.119 & 1.668 & 0.069 & 0.625 \\ 0.150 & 1.161 & 1.778 & 0.064 & 0.650 \\ 0.175 & 1.206 & 1.886 & 0.059 & 0.675 \\ & & & & \\ 0.200 & 1.252 & 1.991 & 0.055 & 0.700 \\ 0.225 & 1.302 & 2.093 & 0.051 & 0.725 \\ 0.250 & 1.358 & 2.192 & 0.046 & 0.750 \\ 0.275 & 1.419 & 2.287 & 0.042 & 0.775 \\ 0.300 & 1.487 & 2.376 & 0.038 & 0.800 \\ 0.325 & 1.562 & 2.457 & 0.034 & 0.825 \\ 0.350 & 1.644 & 2.531 & 0.029 & 0.850 \\ 0.375 & 1.734 & 2.593 & 0.025 & 0.875 \\ 0.400 & 1.832 & 2.643 & 0.021 & 0.900 \\ 0.425 & 1.938 & 2.676 & 0.017 & 0.925 \\ 0.450 & 2.054 & 2.690 & 0.013 & 0.950 \\ 0.475 & 2.178 & 2.682 & 0.009 & 0.975\end{array}$

$E_{0}=0.20 \mathrm{MeV}$

$\begin{array}{cc}\text { POINT } & \text { PLANE } \\ \text { ISOTROPIC } & \text { PERPENDICULAR } \\ \text { SOURCE } & \text { SOURCE }\end{array}$

$\begin{array}{clllc}x & J(x) & J(x) & J(-x) & x \\ 0.000 & 1.000 & 1.237 & 0.237 & 0.500 \\ 0.025 & 1.008 & 1.297 & 0.185 & 0.525 \\ 0.050 & 1.020 & 1.359 & 0.137 & 0.550 \\ 0.075 & 1.038 & 1.424 & 0.093 & 0.575 \\ 0.100 & 1.060 & 1.494 & 0.056 & 0.600 \\ 0.125 & 1.087 & 1.573 & 0.029 & 0.625 \\ 0.150 & 1.118 & 1.663 & 0.015 & 0.650 \\ 0.175 & 1.155 & 1.766 & 0.015 & 0.675 \\ 0.200 & 1.197 & 1.872 & 0.021 & 0.700 \\ 0.225 & 1.244 & 1.974 & 0.026 & 0.725 \\ 0.250 & 1.296 & 2.073 & 0.031 & 0.750 \\ 0.275 & 1.354 & 2.166 & 0.035 & 0.775 \\ 0.300 & 1.417 & 2.254 & 0.038 & 0.800 \\ 0.325 & 1.486 & 2.333 & 0.039 & 0.825 \\ 0.350 & 1.561 & 2.403 & 0.038 & 0.850 \\ 0.375 & 1.642 & 2.461 & 0.036 & 0.875 \\ 0.400 & 1.729 & 2.504 & 0.032 & 0.900 \\ 0.425 & 1.824 & 2.531 & 0.025 & 0.925 \\ 0.450 & 1.925 & 2.537 & 0.016 & 0.950 \\ 0.475 & 2.034 & 2.520 & 0.004 & 0.975\end{array}$

$\begin{array}{cc}\text { POINT } & \text { PLANE } \\ \text { ISOTROPIC } & \text { PERPENDICULAR } \\ \text { SOURCE } & \text { SOURCE }\end{array}$

$\mathrm{J}(\mathrm{x}) \quad \mathrm{J}(\mathrm{x}) \quad \mathrm{J}(-\mathrm{x})$

$\begin{array}{lll}2.310 & 2.649 & 0.005\end{array}$

$\begin{array}{lll}2.448 & 2.591 & 0.002\end{array}$

$2.590 \quad 2.509-0.001$

$2.733 \quad 2.410=0.003$

$\begin{array}{lll}2.871 & 2.299 & -0.004\end{array}$

$\begin{array}{llll}2.996 & 2.175 & =0.004\end{array}$

$\begin{array}{lll}3.095 & 2.036 & -0.003\end{array}$

$\begin{array}{llll}3.151 & 1.880 & -0.001\end{array}$

$\begin{array}{lll}3.146 & 1.705 & 0.002\end{array}$

$\begin{array}{lll}3.057 & 1.509 & 0.005\end{array}$

$\begin{array}{lll}2.868 & 1.293 & 0.006\end{array}$

$\begin{array}{lll}2.577 & 1.057 & 0.006\end{array}$

$\begin{array}{lll}2.203 & 0.809 & 0.004\end{array}$

$\begin{array}{lll}1.762 & 0.559 & 0.000\end{array}$

$\begin{array}{llll}1.269 & 0.329 & -0.002\end{array}$

$\begin{array}{llll}0.762 & 0.147 & -0.004\end{array}$

$\begin{array}{lll}0.325 & 0.040 & -0.002\end{array}$

$0.066 \quad 0.004 \quad-0.000$

$0.002 \quad 0.000=0.000$

$0.000 \quad 0.000=0.000$

$\begin{array}{cc}\text { POINT } & \text { PLANE } \\ \text { ISOTROPIC } & \text { PERPENDICULAR } \\ \text { SOURCE } & \text { SOURCE }\end{array}$

$J(x) \quad J(x) \quad J(-x)$

$\begin{array}{llll}2.150 & 2.480 & -0.007\end{array}$

$\begin{array}{llll}2.272 & 2.418 & -0.015\end{array}$

$\begin{array}{llll}2.399 & 2.338 & -0.017\end{array}$

$\begin{array}{lll}2.525 & 2.244 & -0.013\end{array}$

$\begin{array}{lll}2.645 & 2.138 & -0.008\end{array}$

$\begin{array}{lll}2.750 & 2.022 & -0.002\end{array}$

$\begin{array}{lll}2.834 & 1.892 & 0.002\end{array}$

$\begin{array}{lll}2.885 & 1.748 & 0.005\end{array}$

$\begin{array}{lll}2.891 & 1.586 & 0.007\end{array}$

$\begin{array}{lll}2.836 & 1.404 & 0.008\end{array}$

$\begin{array}{lll}2.704 & 1.201 & 0.007\end{array}$

$\begin{array}{lll}2.476 & 0.979 & 0.005\end{array}$

$\begin{array}{lll}2.137 & 0.744 & 0.002\end{array}$

$\begin{array}{llll}1.688 & 0.509 & -0.001\end{array}$

$\begin{array}{llll}1.159 & 0.294 & -0.004\end{array}$

$\begin{array}{lll}0.625 & 0.127 & -0.004\end{array}$

$\begin{array}{lll}0.216 & 0.032 & -0.002\end{array}$

$\begin{array}{lll}0.029 & 0.003 & -0.000\end{array}$

$\begin{array}{llll}0.000 & 0.000 & -0.000\end{array}$ 
Table 1. Energy Dissipation Function, $J(x)$, and Input Data - Continued

POLYSTYRENE

$E_{0}=0.40 \mathrm{Mev}$

STCPPING POHER

$$
(d E / d r)_{E_{O}}=2.13 \mathrm{Mev}\left(\mathrm{cm}^{2} / g\right)
$$

RESIDUAL RANGE

$$
r_{O}=0.129 \mathrm{~g} / \mathrm{cm}^{2}
$$

OTHER PARAMETERS

$$
\begin{array}{ll}
d_{1}=0.8909 & A_{1}=0.5444 \\
\alpha=9.898 & A_{2}=0.9242 \\
A^{P T I}=0.6689 & A_{3}=-0.7832 \\
{ }_{A} \text { PLP }=0.8040 & A_{4}=0.3145
\end{array}
$$

SCATTERING COEFEICIENTS

$\begin{array}{cc}\ell & s_{\ell} \\ 1 & 0.8092 \\ 2 & 2.243 \\ 3 & 4.188 \\ 4 & 6.576 \\ 5 & 9.361 \\ 6 & 12.51 \\ 7 & 15.98 \\ 8 & 19.76 \\ 9 & 23.82 \\ 10 & 28.14 \\ 11 & 32.70 \\ 12 & 37.49\end{array}$

STOPPING PONER

$$
(\mathrm{dE} / \mathrm{dr})_{E_{O}}=1.89 \mathrm{Mev}\left(\mathrm{cm}^{2} / \mathrm{g}\right)
$$

RESIDUAL RANGE

$$
r_{O}=0.281 \mathrm{~g} / \mathrm{cm}^{2}
$$

OTHER PHRAMETERS

$$
\begin{array}{ll}
\mathrm{d}_{1}=0.8808 & A_{1}=0.4652 \\
{ }^{\alpha}=5.200 & A_{2}=0.9275 \\
{ }_{A} \mathrm{PTI}=0.6502 & A_{3}=-0.6353 \\
{ }_{A} \mathrm{PLP}=0.7821 & A_{4}=0.2426
\end{array}
$$

SCATTERING COEFFICIENTS

\begin{tabular}{cl}
$\ell$ & \multicolumn{1}{c}{$s \ell$} \\
1 & 0.7387 \\
2 & 2.075 \\
3 & 3.906 \\
4 & 6.174 \\
5 & 8.841 \\
6 & 11.87 \\
7 & 15.24 \\
8 & 18.93 \\
9 & 22.91 \\
10 & 27.17 \\
11 & 31.70 \\
12 & 36.48
\end{tabular}

$\begin{array}{cc}\text { POINT } & \text { PLANE } \\ \text { ISOTROPIC } & \text { PERPENDICULAR } \\ \text { SOURCE } & \text { SOURCE }\end{array}$

$\begin{array}{cccc}x & J(x) & J(x) & J(-x) \\ 0.000 & 1.000 & 1.186 & 0.186 \\ 0.025 & 1.006 & 1.237 & 0.142 \\ 0.050 & 1.017 & 1.290 & 0.100 \\ 0.075 & 1.031 & 1.346 & 0.063 \\ 0.100 & 1.049 & 1.407 & 0.032 \\ 0.125 & 1.070 & 1.476 & 0.010 \\ 0.150 & 1.096 & 1.555 & 0.001 \\ 0.175 & 1.125 & 1.646 & 0.001 \\ & & & \\ 0.200 & 1.159 & 1.740 & 0.008 \\ 0.225 & 1.197 & 1.831 & 0.015 \\ 0.250 & 1.238 & 1.918 & 0.020 \\ 0.275 & 1.285 & 2.000 & 0.025 \\ 0.300 & 1.335 & 2.076 & 0.029 \\ 0.325 & 1.390 & 2.145 & 0.031 \\ 0.350 & 1.451 & 2.204 & 0.032 \\ 0.375 & 1.516 & 2.252 & 0.030 \\ & & & \\ 0.400 & 1.586 & 2.286 & 0.026 \\ 0.425 & 1.662 & 2.303 & 0.020 \\ 0.450 & 1.744 & 2.300 & 0.011 \\ 0.475 & 1.832 & 2.277 & 0.000\end{array}$

$E_{0}=0.70 \mathrm{Mev}$

$\begin{array}{cc}\text { POINT } & \text { PLANE } \\ \text { ISOTROPIC } & \text { PERPENDICULAR } \\ \text { SOURCE } & \text { SOURCE }\end{array}$

$\begin{array}{clll}x & J(x) & J(x) & J(-x) \\ 0.000 & 1.000 & 1.140 & 0.140 \\ 0.025 & 1.004 & 1.180 & 0.104 \\ 0.050 & 1.012 & 1.221 & 0.071 \\ 0.075 & 1.023 & 1.266 & 0.042\end{array}$

$\mathrm{x}$

0.500

0.525

0.550

0.575

0.100

0.125

0.150

0.175

$\begin{array}{lll}1.038 & 1.315 & 0.017\end{array}$

$\begin{array}{lll}1.055 & 1.371 & -0.001\end{array}$

1.075

1.098

1.438

1.515

0.200

0.225

1.123

1.596

1.151

1.675

$1.181 \quad 1.750$

1.822

$-0.010$

0.600

0.625

0.650

0.675

0.250

0.275

0.300

0.325

1.213

1. 249

1.888

1. 287

1. 948

0.350

0.375

1.330

2. 000

1.378

2.042

$-0.000$

0.006

0.012

0.017

0.700

0.725

0.750

0.775

0.021

0.024

0.025

0.024

0.400

1.431

2.071

2. 086

0.022

0.425

1.489

1.554

0.475

1.626

2.061

0.016

0.009

0.800

0.825

0.850

0.875

0.900

0.925

0.950

0.975
POINT

ISOTROPIC PERPENDICULAR SOURCE SOURCE

$\mathrm{J}(\mathrm{x})$

$\mathrm{J}(\mathrm{x}) \quad \mathrm{J}(-\mathrm{x})$

1.926

2.026

2.129

2.232

$2.232-0.010$

$2.168-0.016$

$2.090-0.016$

$2.002-0.012$

2.330

2.416

$1.904-0.006$

2.484

$1.798-0.002$

$\begin{array}{ll}1.681 & 0.002\end{array}$

2.524

1.551

0.005

2.526

1.405

0.006

2.476

2. 361

1.243

0.007

2.163

1.063

0.006

1.871

1.482

1.021

0.555

$0.659 \quad 0.002$

$0.450-0.001$

$0.260-0.003$

$0.112-0.003$

$\begin{array}{lll}0.193 & 0.029 & -0.002\end{array}$

0.026

0.000

0.000

$0.002-0.000$

$0.000-0.000$

$0.000-0.000$
POINT PLANE ISOTROPIC PERPENDICULAR SOURCE SOURCE

$\mathrm{J}(\mathrm{x}) \mathrm{J}(\mathrm{x}) \quad \mathrm{J}(-\mathrm{x})$

$\begin{array}{llll}1.705 & 2.019 & -0.009\end{array}$

$\begin{array}{llll}1.792 & 1.960 & -0.014\end{array}$

$\begin{array}{llll}1.884 & 1.889 & -0.015\end{array}$

$\begin{array}{lll}1.980 & 1.808 & -0.011\end{array}$

$\begin{array}{llll}2.074 & 1.719 & -0.006\end{array}$

$2.157 \quad 1.624 \quad-0.002$

$\begin{array}{lll}2.217 & 1.519 & 0.001\end{array}$

$2.245 \quad 1.402 \quad 0.004$

$2.232 \quad 1.273 \quad 0.005$

$2.150 \quad 1.129 \quad 0.006$

$2.050 \quad 0.970 \quad 0.006$

$\begin{array}{lll}1.864 & 0.795 & 0.004\end{array}$

$\begin{array}{lll}1.608 & 0.609 & 0.002\end{array}$

$\begin{array}{llll}1.287 & 0.420 & -0.001\end{array}$

$\begin{array}{llll}0.921 & 0.246 & -0.003\end{array}$

$\begin{array}{llll}0.550 & 0.109 & -0.003\end{array}$

0.240

0.057

$0.029-0.002$

0.003

$0.002-0.000$

$0.000-0.000$

0.000

$0.000-0.000$ 
Table 1. Energy Dissipatioñ Function, $J(x)$, and Input Data - Continued

STOPPING POWER

$$
(\mathrm{dE} / \mathrm{dr})_{\mathrm{E}_{\mathrm{O}}}=1.82 \mathrm{Mev}\left(\mathrm{cm}^{2} / \mathrm{g}\right)
$$

RESIDUAL RANGE

$$
r_{0}=0.443 \mathrm{~g} / \mathrm{cm}^{2}
$$

OTHER PARAMETERS

$$
\begin{array}{ll}
d_{1}=0.8767 & A_{1}=0.4222 \\
a=3.391 & A_{2}=0.8847 \\
{ }_{A}^{\text {PTI }}=0.6288 & A_{3}=-0.4664 \\
{ }_{A}^{\text {PLP }}=0.7565 & A_{4}=0.1595
\end{array}
$$

SCATTERING COEFFICIENTS

\begin{tabular}{rl}
$l$ & \multicolumn{1}{c}{$S_{\ell}$} \\
1 & 0.6770 \\
2 & 1.908 \\
3 & 3.608 \\
4 & 5.726 \\
5 & 8.224 \\
6 & 11.08 \\
7 & 14.26 \\
8 & 17.75 \\
9 & 21.54 \\
10 & 25.60 \\
11 & 29.93 \\
12 & 34.51
\end{tabular}

STOPPING POWER

$$
(d E / d r)_{E_{0}}=1.79 \mathrm{Mev}\left(\mathrm{cm}^{2} / g\right)
$$

RESIDUAL RANGE

$$
r_{0}=1.00 \mathrm{~g} / \mathrm{cm}^{2}
$$

OTHER PARAMETERS

$$
\begin{array}{ll}
d_{1}=0.8960 & A_{1}=0.3442 \\
d=1.370 & A_{2}=0.9492 \\
{ }_{A}{ }^{P T}=0.5780 & A_{3}=-0.4675 \\
{ }_{A}^{P L P}=0.6926 & A_{4}=0.1740
\end{array}
$$

SCATTERING COEFFICIENTS

$$
\begin{array}{cc}
l & \multicolumn{1}{c}{s_{\ell}} \\
1 & 0.5179 \\
2 & 1.477 \\
3 & 2.814 \\
4 & 4.492 \\
5 & 6.486 \\
6 & 8.779 \\
7 & 11.35 \\
8 & 14.19 \\
9 & 17.29 \\
10 & 20.63 \\
11 & 24.20 \\
12 & 28.00
\end{array}
$$

POLYSTYRENE

\begin{tabular}{|c|c|c|c|c|c|c|c|}
\hline $\mathbf{x}$ & $J(x)$ & $J(x)$ & $J(-x)$ & $x$ & $J(x)$ & $J(x)$ & $J(-x)$ \\
\hline $\begin{array}{l}0.000 \\
0.025 \\
0.050 \\
0.075\end{array}$ & $\begin{array}{l}1.000 \\
1.000 \\
1.002 \\
1.006\end{array}$ & $\begin{array}{l}1.058 \\
1.074 \\
1.092 \\
1.112\end{array}$ & $\begin{array}{l}0.058 \\
0.043 \\
0.028 \\
0.015\end{array}$ & $\begin{array}{l}0.500 \\
0.525 \\
0.550 \\
0.575\end{array}$ & $\begin{array}{l}1.361 \\
1.403 \\
1.448 \\
1.495\end{array}$ & $\begin{array}{l}1.698 \\
1.669 \\
1.623 \\
1.562\end{array}$ & $\begin{array}{r}0.001 \\
-0.004 \\
-0.008 \\
-0.010\end{array}$ \\
\hline $\begin{array}{l}0.100 \\
0.125 \\
0.150 \\
0.175\end{array}$ & $\begin{array}{l}1.011 \\
1.018 \\
1.027 \\
1.038\end{array}$ & $\begin{array}{l}1.136 \\
1.165 \\
1.203 \\
1.251\end{array}$ & $\begin{array}{r}0.004 \\
-0.005 \\
-0.009 \\
-0.009\end{array}$ & $\begin{array}{l}0.600 \\
0.625 \\
0.650 \\
0.675\end{array}$ & $\begin{array}{l}1.544 \\
1.594 \\
1.644 \\
1.688\end{array}$ & $\begin{array}{l}1.490 \\
1.412 \\
1.326 \\
1.233\end{array}$ & $\begin{array}{r}-0.008 \\
-0.005 \\
-0.002 \\
0.001\end{array}$ \\
\hline $\begin{array}{l}0.200 \\
0.225 \\
0.250 \\
0.275\end{array}$ & $\begin{array}{l}1.050 \\
1.064 \\
1.080 \\
1.098\end{array}$ & $\begin{array}{l}1.305 \\
1.378 \\
1.410 \\
1.461\end{array}$ & $\begin{array}{r}-0.005 \\
-0.002 \\
0.001 \\
0.003\end{array}$ & $\begin{array}{l}0.700 \\
0.725 \\
0.750 \\
0.775\end{array}$ & $\begin{array}{l}1.720 \\
1.725 \\
1.687 \\
1.589\end{array}$ & $\begin{array}{l}1.131 \\
1.017 \\
0.889 \\
0.748\end{array}$ & $\begin{array}{l}0.003 \\
0.004 \\
0.005 \\
0.004\end{array}$ \\
\hline $\begin{array}{l}0.300 \\
0.325 \\
0.350 \\
0.375\end{array}$ & $\begin{array}{l}1.118 \\
1.140 \\
1.164 \\
1.191\end{array}$ & $\begin{array}{l}1.510 \\
1.556 \\
1.599 \\
1.637\end{array}$ & $\begin{array}{l}0.006 \\
0.008 \\
0.009 \\
0.010\end{array}$ & $\begin{array}{l}0.800 \\
0.825 \\
0.850 \\
0.875\end{array}$ & $\begin{array}{l}1.415 \\
1.157 \\
0.823 \\
0.461\end{array}$ & $\begin{array}{l}0.593 \\
0.430 \\
0.269 \\
0.131\end{array}$ & $\begin{array}{r}0.002 \\
0.000 \\
-0.002 \\
-0.003\end{array}$ \\
\hline $\begin{array}{l}0.400 \\
0.425 \\
0.450 \\
0.475\end{array}$ & $\begin{array}{l}1.220 \\
1.251 \\
1.285 \\
1.322\end{array}$ & $\begin{array}{l}1.669 \\
1.693 \\
1.708 \\
1.710\end{array}$ & $\begin{array}{l}0.010 \\
0.010 \\
0.008 \\
0.005\end{array}$ & $\begin{array}{l}0.900 \\
0.925 \\
0.950 \\
0.975\end{array}$ & $\begin{array}{l}0.167 \\
0.026 \\
0.001 \\
0.001\end{array}$ & $\begin{array}{l}0.040 \\
0.004 \\
0.000 \\
0.000\end{array}$ & $\begin{array}{l}-0.002 \\
-0.000 \\
-0.000 \\
-0.000\end{array}$ \\
\hline
\end{tabular}

$$
E_{0}=1.00 \mathrm{Mev}
$$

$\begin{array}{cc}\text { POINT } & \text { PLANE } \\ \text { ISOTROPIC } & \text { PERPENDICULAR } \\ \text { SOURCE } & \text { SOURCE }\end{array}$

$\begin{array}{cccc}\mathbf{x} & \mathrm{J}(\mathbf{x}) & \mathrm{J}(\mathbf{x}) & \mathrm{J}(-\mathbf{x}) \\ 0.000 & 1.000 & 1.110 & 0.110 \\ 0.025 & 1.003 & 1.142 & 0.081 \\ 0.050 & 1.008 & 1.176 & 0.054 \\ 0.075 & 1.016 & 1.212 & 0.030 \\ & & & \\ 0.100 & 1.026 & 1.253 & 0.010 \\ 0.125 & 1.039 & 1.300 & -0.005 \\ 0.150 & 1.054 & 1.357 & -0.012 \\ 0.175 & 1.072 & 1.424 & -0.010 \\ & & & \\ 0.200 & 1.093 & 1.496 & -0.003 \\ 0.225 & 1.117 & 1.566 & 0.003 \\ 0.250 & 1.143 & 1.634 & 0.008 \\ 0.275 & 1.172 & 1.698 & 0.013 \\ & & & \\ 0.300 & 1.204 & 1.758 & 0.017 \\ 0.325 & 1.239 & 1.812 & 0.020 \\ 0.350 & 1.278 & 1.860 & 0.021 \\ 0.375 & 1.319 & 1.899 & 0.021 \\ 0.400 & 1.364 & 1.928 & 0.020 \\ 0.425 & 1.412 & 1.943 & 0.016 \\ 0.450 & 1.463 & 1.943 & 0.009 \\ 0.475 & 1.517 & 1.925 & 0.001\end{array}$

$x$
0.500
0.525
0.550
0.575

POINT ISOTROPIC PERPENDICULAR SOURCE SOURCE

$\begin{array}{llr}J(x) & J(x) & J(-x) \\ 1.575 & 1.889 & -0.007 \\ 1.636 & 1.836 & -0.013 \\ 1.700 & 1.770 & -0.014 \\ 1.766 & 1.696 & -0.011 \\ & & \\ 1.834 & 1.614 & -0.007 \\ 1.900 & 1.526 & -0.003 \\ 1.962 & 1.430 & 0.001 \\ 2.013 & 1.324 & 0.003 \\ & & \\ 2.042 & 1.206 & 0.065 \\ 2.032 & 1.074 & 0.006 \\ 1.963 & 0.928 & 0.005 \\ 1.818 & 0.767 & 0.004 \\ & & \\ 1.582 & 0.594 & 0.002 \\ 1.253 & 0.416 & -0.001 \\ 0.854 & 0.249 & -0.003 \\ 0.450 & 0.113 & -0.003 \\ & & \\ 0.150 & 0.031 & -0.002 \\ 0.023 & 0.003 & -0.000 \\ 0.006 & 0.000 & -0.000 \\ 0.010 & 0.000 & -0.000\end{array}$

$E_{0}=2.00 \mathrm{MeV}$

$\begin{array}{cc}\text { POTNT } & \text { PLANE } \\ \text { ISOTROPIC } & \text { PERPENDICULAR } \\ \text { SOURCE } & \text { SOURCE }\end{array}$

0.600

0.625

0.650

0.675

0.700

0.725

0.750

0.775

0.800

0.825

0.850

0.875

0.900

0.925

0.950

0.975

$0.000-0.000$

$\begin{array}{cc}\text { POINT } & \text { PLANE } \\ \text { ISOTROPIC } & \text { PERPENDICULAR } \\ \text { SOURCE } & \text { SOURCE }\end{array}$


Table 1. Energy Disaipation Function, J(x), and Input Data - Continued

STOPPING POVER

$$
(d E / d r)_{E_{0}}=1.84 \operatorname{Mov}\left(\mathrm{cm}^{2} / g\right)
$$

RESIDUAL RANGE

$$
r_{0}=2.10 \mathrm{~g} / \mathrm{cm}^{2}
$$

OTHER PARANETERS

$$
\begin{array}{ll}
d_{1}=0.9138 & A_{1}=0.2982 \\
a=0.6231 & A_{2}=0.9289 \\
A^{P T I}=0.4952 & A_{3}=-0.3859 \\
A^{P L P}=0.5886 & A_{4}=0.1588
\end{array}
$$

SCATTERING COEFICIENTS

$$
\begin{array}{cc}
l & s_{l} \\
1 & 0.3508 \\
2 & 1.006 \\
3 & 1.927 \\
4 & 3.092 \\
5 & 4.394 \\
6 & 6.093 \\
7 & 7.909 \\
8 & 9.922 \\
9 & 12.13 \\
10 & 14.51 \\
11 & 17.08 \\
12 & 19.82
\end{array}
$$

STOPPING POWER

$$
(d E / d r)_{E_{O}}=1.94 \operatorname{Mev}\left(\mathrm{cm}^{2} / g\right)
$$

RESIDUAL RANGE

$$
r_{0}=5.26 \mathrm{~g} / \mathrm{cm}^{2}
$$

OTHER' PARAMETERS

$$
\begin{array}{ll}
d_{1}=0.9486 & A_{1}=0.2476 \\
a^{\text {PTI }}=0.2341 & A_{2}=1.040 \\
A^{\text {PTI }}=0.3688 & A_{3}=-0.6041 \\
A^{\text {PLP }}=0.4265 & A_{4}=0.3168
\end{array}
$$

\section{SCATTERING COEFFICIENTS}

\begin{tabular}{cc}
$l$ & \multicolumn{1}{c}{$s_{l}$} \\
1 & 0.1799 \\
2 & 0.5194 \\
3 & 1.001 \\
4 & 1.614 \\
5 & 2.352 \\
6 & 3.269 \\
7 & 4.182 \\
8 & 5.265 \\
9 & 6.457 \\
10 & 7.754 \\
11 & 9.153 \\
12 & 10.65
\end{tabular}

\section{POLYSTYRENE}

$$
E_{0}=4.00 \mathrm{MeV}
$$

$\begin{array}{cc}\text { POINT } & \text { PLANE } \\ \text { ISOTROPIC } & \text { PERPENDICULAR } \\ \text { SOURCE } & \text { SOURCE }\end{array}$

$\begin{array}{cccc}x & J(x) & J(x) & J(-x) \\ 0.000 & 1.000 & 1.025 & 0.025 \\ 0.025 & 0.999 & 1.030 & 0.019 \\ 0.050 & 0.999 & 1.036 & 0.014 \\ 0.075 & 1.000 & 1.045 & 0.009 \\ & & & \\ 0.100 & 1.002 & 1.056 & 0.005 \\ 0.125 & 1.006 & 1.070 & 0.001 \\ 0.150 & 1.010 & 1.090 & -0.002 \\ 0.175 & 1.015 & 1.117 & -0.002 \\ & & & \\ 0.200 & 1.021 & 1.150 & -0.002 \\ 0.225 & 1.028 & 1.183 & -0.001 \\ 0.250 & 1.036 & 1.217 & -0.000 \\ 0.275 & 1.046 & 1.251 & 0.000 \\ 0.300 & 1.057 & 1.285 & 0.001 \\ 0.325 & 1.069 & 1.320 & 0.001 \\ 0.350 & 1.082 & 1.353 & 0.002 \\ 0.375 & 1.097 & 1.386 & 0.002 \\ 0.400 & 1.114 & 1.418 & 0.002 \\ 0.425 & 1.132 & 1.447 & 0.003 \\ 0.450 & 1.152 & 1.472 & 0.003 \\ 0.475 & 1.173 & 1.493 & 0.002\end{array}$

$$
E_{0}=10.00 \mathrm{Mev}
$$

$\begin{array}{cc}\text { POINT } & \text { PLANE } \\ \text { ISOTROPIC } & \text { PERPENDICULAR } \\ \text { SOURCE } & \text { SOURCE }\end{array}$

$x$

0.000

0.025

0.050

0.075

$J(x) \quad J(x)$

0.100

0.125

0.150

0.175

1.000

1.008

0.9991 .009

0.008

0.999

1.011

0.007

0.999

1.013

0.006

1.000

1.017

1. 001

1.022

1.003

1.028

0.004

1.006

1.035

0.003

0.002

0.200

1.008

$1.044-0.000$

0.225

0.250

1.011

$1.054-0.001$

1.012

$1.065-0.002$

0.275

1.013

$1.078-0.002$

0.300

$\begin{array}{lll}1.015 & 1.092 & -0.001\end{array}$

0.325

0.350

1.017

1.108

$-0.001$

1. 019

1.125

$-0.001$

0.375

1.021

$1.143-0.001$

0.400

$$
\begin{array}{lll}
1.024 & 1.162 & -0.000
\end{array}
$$

0.425

\subsection{8}

1.182

0.000

1.033

1.202

0.000

0.475
1.040
POINT

ISOTROPIC

SOURCE

$J(-x)$

0.500

0.525

0.550

0.575

0.600

0.625

0.650

0.675

0.700

0.725

0.750

0.775

0.800

0.825

0.850

0.875

0.900

0.925

0.950

0.975

$1.22 \varepsilon$

1.102

0.289

0.577

0.229

0.024

0.000
POINT

ISOTROPIC PERPENDICULAR SOURCE SOURCE

$\begin{array}{llr}J(x) & J(x) & J(-x) \\ 1.197 & 1.508 & 0.002 \\ 1.223 & 1.514 & 0.001 \\ 1.250 & 1.509 & 0.000 \\ 1.280 & 1.490 & -0.001 \\ & & \\ 1.312 & 1.454 & -0.002 \\ 1.346 & 1.401 & -0.003 \\ 1.381 & 1.331 & -0.003 \\ 1.418 & 1.250 & -0.002 \\ & & \\ 1.453 & 1.160 & -0.000 \\ 1.484 & 1.060 & 0.001 \\ 1.501 & 0.949 & 0.002 \\ 1.487 & 0.824 & 0.002 \\ & & \\ 1.410 & 0.684 & 0.002 \\ 1.230 & 0.528 & 0.001 \\ 0.933 & 0.363 & -0.000 \\ 0.560 & 0.203 & -0.001 \\ & & \\ 0.224 & 0.077 & -0.001 \\ 0.044 & 0.013 & -0.000 \\ 0.002 & 0.000 & 0.000 \\ 0.000 & 0.000 & 0.000\end{array}$

PLANE

PERPENDICULAR SOURCE

$\mathrm{J}(\mathrm{x}) \mathrm{J}(\mathrm{x}) \quad \mathrm{J}(-\mathrm{x})$

$1.049 \quad 1.245 \quad 0.001$

$1.061 \quad 1.266 \quad 0.001$

$\begin{array}{lll}1.078 & 1.287 & 0.001\end{array}$

$\begin{array}{lll}1.100 & 1.305 & 0.001\end{array}$

$\begin{array}{lll}1.128 & 1.319 & 0.001\end{array}$

$\begin{array}{lll}1.159 & 1.328 & 0.001\end{array}$

$\begin{array}{lll}1.192 & 1.328 & 0.001\end{array}$

$\begin{array}{lll}1.225 & 1.316 & -0.000\end{array}$

$\begin{array}{lll}1.257 & 1.286 & -0.001\end{array}$

$\begin{array}{lll}1.285 & 1.233 & -0.002\end{array}$

$\begin{array}{lll}1.305 & 1.152 & -0.003\end{array}$

$\begin{array}{lll}1.310 & 1.042-0.002\end{array}$

$\begin{array}{lll}1.289 & 0.910 & 0.000\end{array}$

0.761

0.596

0.002

0.413

0.003
$0.227-0.001$

$0.074-0.002$

$0.066-0.000$

$0.000 \quad 0.000$ 
Table 2. Spatial Moments; Plane Isotropic Source

$$
p=-1 / 2
$$$$
1 / 2
$$

$3 / 2$

$5 / 2$

\section{MEV $=0.025 \quad Z=6$.}

$0.200623 E 01$ $0.207134 E=00$

$0.554574 \varepsilon-01$

$0.203295 E-01$

$0.882493 E-02$

$0.427186 E-02$

$0.223431 E=02$

$0.665423 E \quad 00$ $0.353090 E-01$ $0.665209 E-02$ $0.190310 E-02$ $0.680553 E-03$ $0.280751 E-03$ $0.128119 E-03$

$0.398403 E-00$ $0.128270 E-01$ $0.179023 E-02$ $0.410774 E-03$ $0.123130 \mathrm{E}-03$ $0.438166 E=04$ $0.176028 E-04$ MEV $=0.05$

$z=6$.

- 195082E OI $0.200989 E=00$ $0.536731 E=01$ $0.195981 E-01$ $0.846725 E-02$ $0.407747 E-02$

$0.212097 E=02$

$0.676671 E \quad 00$ $0.364758 E-01$ $0.690895 E=02$ $0.197824 \mathrm{E}-02$ $0.706352 \mathrm{E}-03$ $0.290559 E-03$ $0.132104 \mathrm{E}-03$ MEV $=0.10$ $z=6$.

0 $0.208707 E-00$ $0.565488 E=01$ $0.208796 \mathrm{E}=01$ . - $910314 \mathrm{E}=02$ $0.441732 \mathrm{E}=02$ $0.231293 E=02$ $0.362987 E-01$ $0.692826 \mathrm{E}-02$ $0.199788 \mathrm{E}-02$ $0.717894 \mathrm{E}-0$ $0.296977 \mathrm{E}-03$ $0.135706 E-03$ $M E V=0.20$

$z=6$.
0

$0.196614 E \quad 01$ $0.208310 \varepsilon-00$ $0.566645 E-01$ $0.209639 E=01$ $0.914741 E-02$ $0.443911 E=02$

$0.232330 E=02$
$0.412986 E-00$ $0.137752 E=01$ $0.195421 E-02$ $0.452009 \mathrm{E}=03$ $0.135979 \mathrm{E}-03$ $0.484358 E-04$ $0.194447 E-04$

$0.404333 E-00$ $0.133567 \mathrm{E}=0$ $0.189422 \mathrm{E}-02$ $0.438975 E-03$ 0. $132406 \mathrm{E}-0$ $.472980 \mathrm{E}-0$ $0.190424 E-04$

$0.408867 E-00$ $0.673518 \mathrm{E} 00$ $0.368629 \varepsilon=01$ $0.707560 \mathrm{E}-02$ $0.204653 E-02$ $0.736484 E-03$ $0.304840 E-03$ $0.139289 \mathrm{E}-03$

$M E V=0.40$ $2=6$.

0 $0.193695 E 01$ $0.207728 E-00$ $0.570670 E-01$ $0.212698 E-01$ $0.933468 E-02$ $0.933468 \mathrm{E}-02$ $0.455114 \mathrm{E}-02$
$0.239109 \mathrm{E}-02$

0.679556 E 00 $0.378685 E-01$ $0.736060 E-02$ $0.214846 E-02$ $0.778572 \mathrm{E}-03$ $0.324045 E-03$ $0.148731 \mathrm{E}-03$ $M E V=0.70 \quad Z=6$.

$0.188794 \mathrm{E} \quad 01$ $0.205297 E-00$ $0.571854 E-01$ $0.215602 E-01$ $0.955452 E-02$ $0.469773 E-02$ $0.248656 E-02$

$0.689981 E \quad 00$ $0.394641 \mathrm{E}-01$ $0.781980 \mathrm{OE}-02$ $0.231680 E-02$ $0.849804 E-03$ $0.357313 E=03$ $0.165449 E-03$ $0.137127 E=01$ $0.196121 E-02$ $0.456729 E-03$ $0.138155 \mathrm{E}-03$ $0.494290 E-04$ $0.199145 E-04$

$0.416776 \mathrm{E}-00$ $0.143514 E=01$ $0.208820 \varepsilon-02$ $0.492294 E-03$ $0.150292 E-03$ $0.541621 E-04$ $0.219492 \mathrm{E}-04$

$0.430648 E-00$ $0.154562 \mathrm{E}-01$ $0.231200 E-02$ $0.556385 E-03$ $0.172641 \mathrm{E}-0$ O. 172641 $0.258421 E=04$ MEV $=1.00$ $z=6$. $0.202286 E=00$ $0.570851 E-01$ $0.217698 E-01$ $0.974471 E-02$ $0.483431 E=02$

$0.257967 \mathrm{E}-02$ MEV $=2.00$

$0.168856 E$ OI $0.189832 \mathrm{E}=00$ $0.553143 \mathrm{E}-01$ $0.217104 E-01$ $0.996824 E-02$ $0.505878 E-02$ $0.275556 E-02$
.700595E 00 $0.410641 E=01$ $0.829187 \mathrm{E}=02$ $0.249421 \mathrm{E}-02$ $0.926540 E-03$ $0.393849 E-03$

$0.184127 \mathrm{E}-03$

$z=6$

$0.445051 E-00$ $0.166306 E-01$ $0.255706 \mathrm{E}-02$ $0.628434 E-03$ $0.198337 \mathrm{E}-03$ $0.734754 E-04$ $0.304857 E-04$

$0.736579 E$ OD $0.464625 \mathrm{E}-01$ $0.990914 \mathrm{E}-02$ $0.311271 \mathrm{E}-02$ $0.119848 \mathrm{E}-02$ $0.525255 E=03$ $0.252211 \mathrm{E}-03$
$0.284237 \mathrm{E}-0$ $0.613529 E=0$ $0.659319 E-03$ $0.124023 E-03$ $0.316172 \mathrm{E}-04$ $0.980682 \mathrm{E}-05$ 0.349565 E- 05

$0.297812 \mathrm{E}=00$ $0.676858 E=02$ $0.747400 \mathrm{E}-03$ $0.142820 \mathrm{E}-03$ $0.367520 E-04$ $0.114620 \mathrm{E}-04$ $0.409748 E=05$

$0.289734 \mathrm{E}-00$ $0.644678 E-02$ $0.706300 E-03$ $0.134514 E-03$ $0.345695 E-04$ $0.107789 E-04$ 0.385476 E-OS

$0.293959 E-00$ 0.666916 E-02 $0.739035 \mathrm{E}-03$ $0.141740 E-03$ $0.365888 \mathrm{E}-04$ $0.114406 E-04$ $0.409837 E-05$

$0.301370 E=00$ $0.707140 \mathrm{E}-02$ $0.801071 E-03$ $0.156075 E=03$ $0.407707 E-04$ $0.128676 E-04$ $0.464441 E=05$

$0.314500 E-00$ $0.779301 \mathrm{E}-02$ $0.915316 E-03$ $0.183165 E-03$ $0.488608 E-04$ $0.156866 E=04$

$0.328306 E-00$ $0.858396 \mathrm{E}-02$ $0.104529 E=02$ $0.214957 \mathrm{E}-03$ $0.586055 E-04$ $0.191584 E-04$ C. $712373 E-05$

$0.378611 E-00$ $0.117631 \mathrm{E}-0$ $0.160502 E-02$ $0.359467 \mathrm{E}=03$ $0.104899 \mathrm{E}-03$ $0.362749 \mathrm{E}-04$ $0.141477 \mathrm{E}-04$

$0.220907 E-00$ $0.341688 E-02$ $0.291312 E-03$ $0.457380 E-04$ $0.273872 E-05$ $0.872190 E=06$

$0.233046 E=00$ $0.384528 E=02$ $0.340233 E-03$ $0.546787 \mathrm{E}=0$ $0.121866 \mathrm{E}-04$ $0.335871 E-05$ $0.107693 E=05$

$0.225809 \mathrm{E}-00$ $0.361474 E-02$ $0.315200 E-03$ $0.502319 E-04$ $0.11370 E=04$ $0.305905 E=05$ $0.978681 \mathrm{E}=06$

$0.229589 \mathrm{E}-00$ $0.376064 E-02$ $0.332597 \mathrm{E}=03$ $0.534919 E-04$ $0.119326 E-04$ $0.329124 E-05$ $0.105592 E-05$

$0.236247 E-00$ $0.402638 E-02$ 
Toble 2. Spotial Maments, Plane Isotropic Source-Continued

n $p=-1 / 2$

$1 / 2$

$3 / 2$

$5 / 2$

$7 / 2$

$9 / 2$

$11 / 2$

$Z=6$.

$0.155943 E-00$

$0.512465 E-01$

$0.226702 E-01$

$0.116375 \bar{E}-0.1$

$0.654805 E-02$

$0.654805 E-02$
$0.392541 E-02$

MEV $=0.025$

$0.783300 E$ OO

$0.545245 E-01$

$0.125918 E-01$

$0.422722 \mathrm{E}-02$

$0.172299 E-02$

$0.793759 E-03$

$0.398476 \dot{E}-03$

. $67490 E$ OO $284224 E-01$ $0.534768 E-02$ $0.153779 E-02$ $0.552072 E-03$ $0.228121 E-0$ $0.104063 \bar{E}-03$

6.

$0.850168 E 00$ $0.682416 E-0$ $0.178329 E-01$ $0.667148 E-02$ $0.299340 E-02$ $0.1516145 E-03$

$0.680953 E 00$ $0.431202 E=01$ $0.967636 E-02$ $0.322119 E-02$ $0.131213 E-02$ $0.006114 E-03$ $0.305548 \mathrm{E}-03$

$Z=13$.

$0.203906 E$ OI

$0.151044 E=00$

$0.301676 E-01$

$0.856798 E-02$

$0.296188 E-02$

$0.116552 E-0$

$0.503584 E-03$

$0.658958 E 00$

$0.269695 E-01$

$0.390208 E-02$

$0.878561 E-03$

$0.252766 E-03$

$0.854027 E-04$

$0.323834 E-04$

$0.390161 E-00$ $0.100702 E-01$ $0.110239 E-02$ $0.201491 \mathrm{E}-03$ $0.489806 \mathrm{E}-04$ $0.143590 E=04$ $0.481525 E-05$ MEV $=0.05$ $z=13$.

$0.198057 E$ OI $0.144237 E-00$ $0.282923 \varepsilon=01$ $0.788767 E-02$ $0.267656 E-02$ $0.103406 E-02$ $0.438749 E-03$

\section{$0.670578 E \quad 00$ $0.275627 E-01$ $0.395426 E-02$ $0.878980 E-03$ $0.249209 E-03$ $0.829052 E-04$ \\ $0.309403 E-04$} $M E V=0.10$ $z=13$.

$0.198161 E 01$
$0.146022 E-00$
$0.288370 E-01$
$0.807240 E-02$
$0.274594 E-02$
$0.106227 E-02$
$0.450951 E-03$

$0.670368 E 00$ $0.278011 E-01$

$0.401017 E-02$

$0.894246 E-03$

. $253993 E-03$

$.845683 E-04$

$0.315657 \mathrm{E}-04$

$0.405047 E-00$ $0.107315 E-01$ $0.117931 E-02$ $0.214536 E=03$ $0.516923 E-04$ $0.149865 E-04$ $0.496351 E-05$ $0.107991 E-01$ $0.119195 E-02$ $0.217362 E-03$ 0 $0.524374 E=04$ $0.503610 E=05$
MEV $=0.20$

$0.196899 E \quad 01$ $0.146858 E-00$ $0.292205 E-01$ $0.821866 E-02$ $0.280425 E-02$ $0.108691 E=02$ $0.461912 E-03$

\section{$0.672936 \mathrm{E} 00$} $0.282834 E-01$ $0.411316 E-02$ $0.921919 E-03$ 0.26271 YE -03 $0.876569 E-04$ $0.327586 E-04$ $z=13$.

0.193635 E O $0.147251 E=00$ $0.297308 E-01$ $0.845498 \mathrm{E}-02$ $0.291013 E-02$ $0.113599 \mathrm{E}-02$ $0.485042 E-03$
$0.408108 E-00$ $0.110631 E-01$ $0.123302 E-02$ $0.226242 E-03$ $0.548013 E-04$ $0.159367 E-04$ $0.528603 E-05$
$0.452743 E-00$ $0.175219 E-01$ $0.278311 E-02$ $0.702075 \mathrm{E}-03$ $0.226029 E-03$ $0.849868 E-04$ $0.356521 E-04$

$0.579212 E 00$ $0.305003 E-01$ $0.605345 \mathrm{E}-02$ $0.183102 E-02$ $0.688322 E-03$ $0.296468 E-03$ $0.140390 E-03$

$0.276646 E=00$ $0.490342 E-02$ $0.420088 E-03$ $0.035733 E-04$ $0.132303 E-04$ $0.339694 E-05$ $0.101455 E-05$

$0.290399 E=00$ $0.537920 E-02$ $0.468200 E-03$ $0.711238 E-04$ $0.147654 E-0$ $0.376806 E-0$ $0.111598 E-05$

$0.290147 E-00$ $0.540427 \mathrm{E}-02$ $0.472056 E-03$ $0.718395 E-04$ $0.149249 E-04$ $0.380881 E=0 S$ $0.112746 E=05$

$0.293251 E-00$ $0.556312 E-02$ $0.491523 E-03$ $0.753583 E-04$ $0.157344 E-04$ $0.402910 E-05$ $0.119540 E-05$

$0.301525 E-00$ $0.596068 E-02$ $0.541517 E-03$ $0.179663 E-04$ $0.179663 E-04$ $0.139605 E-05$

$0.316764 E-00$ $0.670338 E-02$ $0.638412 E-03$ $0.103493 E-03$ $0.225748 E=04$
$0.599017 E=05$ $0.599017 E=05$
$0.379255 E-00$ $0.118231 \mathrm{E}-01$ $0.162094 E-02$ $0.364277 \mathrm{E}=03$ $0.106431 E-03$ $0.367706 E-04$ $0.143024 E-04$

$0.508263 E 00$ $0.229145 E-01$ $0.409141 E-02$ $0.113848 E-02$ $0.398903 E-03$ $1540 E=03$ $0.723784 E-04$

$0.214169 E-00$ $0.276502 E-02$ $0.190391 E-03$ $0.242509 E=04$ $0.436988 E-05$ $0.990875 E-06$ $0.265250 E=06$

$0.226403 E-00$ $0.309902 E-02$ $0.219179 E-03$ $0.282572 \mathrm{E}-04$ $0.511241 E-05$ $0.115824 E-05$ $0.308789 E-06$

$0.226177 E-00$ $0.310972 E-02$ $0.220571 E-03$ $0.284736 E-04$ $0.116707 E-05$ $0.310888 E-06$

$0.228954 E-00$ $0.321259 E-02$ $0.230870 E-03$ $0.300634 E-04$ $0.547370 E-05$ $0.124493 E-05$ $0.332619 E-06$

$0.236386 E-00$ $0.347761 E-02$ $0.258190 \mathrm{E}-03$ $0.344369 E-04$ $0.638735 E-05$ $0.147446 E-05$ $0.398775 E-06$

$0.250183 \varepsilon=00$ $0.398536 \mathrm{E}-02$ $0.312868 E-03$ $0.435351 E-04$ $0.835180 E-05$ 0.18 $0.198224 E-05$
$0.548839 E-06$

$0.327453 E=00$ $0.845708 E-02$ $0.101666 \mathrm{E}-02$ $0.2058195-03$ $0.550514 E=04$ $0.176029 E-0$ $0.638771 E-05$

$.454913 E-00$ $0.179008 E-01$ $0.290878 E-02$ $0.750981 E-03$ $0.246943 E-03$ $0.945738 E-04$ $0.402988 E-04$

0.174667E-00 $0.171482 E-02$ $0.969603 E-04$ $0.105396 E-04$ $0.166067 E-05$ 
Toble 2. Spotial Moments, Plone Isotropic Source - Continued

$5 / 2$

$7 / 2$

$9 / 2$

$11 / 2$ $z=13$.

$M E V=1.00$
$0.704539 E \quad 00$ $0.512369 E-02$ $0.121500 E-02$ $0.362352 E-03$ $0.125598 E-03$ $0.485042 E-04$ $0.327330 E-01$
$0.450476 E-00$ $0.140759 E-01$ $0.173089 E-02$ $0.341890 E-03$ $0.877958 E-04$ $0.267912 E-04$ $0.925672 E-05$ $Z=13$.

$$
M E V=2.00
$$

$0.739576 E 00$ $0.380482 E-01$ $0.645579 E-02$ $0.163376 E-02$ $0.514562 E-03$ $0.514562 E-03$ $0.753012 E-04$

$0.317098 E-01$

$0.981214 E-02$

$0.363200 E-02$

$0.151186 \mathrm{E}-02$

$0 \quad z=130$

$$
M E V=4.00
$$

00

$0.150159 \mathrm{E} \mathrm{Ol}$

$0.787877 E$ OO

$0.464647 E-01$

$0.882940 E-02$

$0.245461 E-02$

$0.837663 E-03$

$0.326544 E-03$

$0.435153 \mathrm{E}-02$

$0.140016 E-03$ $0.403105 E-02$$$
z=13 \text {. }
$$

$0.500452 E 00$ $0.181241 \mathrm{E}-01$ $0.248215 E-02$ $0.533581 E-03$ $0.146902 E-03$ $0.146902 E=03$ $0.475634 E=04$
$0.173055 E-04$

$0.574821 E 00$ $0.253533 E-01$ $0.977699 \mathrm{E}-03$ $0.297898 E-03$

$0.105281 E-03$ $0.413833 E-04$ $z=13$.
$0.691847 E \quad 00$ $0.403513 E-01$ $0.803401 E-02$ $0.235675 E-02$ $0.846984 E-03$ $0.346458 E=03$ $0.155339 E-03$ $z=29$.

0

$0.216947 E$ OI $0.996314 E-01$ $0.131741 \mathrm{E}-01$ $0.261674 E-02$ $0.657843 E-03$ $0.193843 E-03$ $0.641671 E-04$

$0.856174 E$ $0.615027 E-01$ $0.452050 E-02$ $0.176588 E-02$ $0.776773 E-03$ $0.371636 E-03$ 0.55436 dE $=04$ $0.1411: 9 E=04$ $0.412-18 E-05$

$0.360038 E-00$ $0.618359 E-02$ $0.459264 E-03$ $0.594974 E-04$ $0.10623 E=04$ $0.234 \% 76 E-05$ $0.60: 781 E-06$ MEV $=0.05$ $z=29$.

0 $0.846654 E-01$ $0.101974 E-01$ $0.185200 E-02$ $0.427152 \mathrm{E}=03$ $0.427152 E=03$ $0.353668 E-04$

$0 .-00475 E 00$ $0.168527 E-01$ G, $153258 \mathrm{E}-02$ . $226240 E-C$. $0.442080 E-04$ $0.104336 \bar{E}-04$ $0.282725 E-05$ $0.678815 E-02$ $0.484238 E-03$ $0.593475 E-04$ $0.995816 E-05$ $0.206502 E-05$ $0.499959 E-06$ MEV - $0.10 \quad z=29$.

0.201498 E Ol

$0.663686 E$ OO

$0.396179 \varepsilon=0$ $0.681848 \mathrm{E} \cdot 02$ 0.4787045 .23 $0.575897 k-04$ $0.947584 E-05$ $0.192605 E-05$ $0.456992 E-06$

0 $0.198355 E$ OI $0.806730 E-01$ $0.943628 E-02$ $0.166107 E-02$ $0.166107 E=02$
$0.370971 E=03$ $0.370971 E=03$
$0.973453 E=04$ $0.287634 \mathrm{E}=04$ $Z=29$.

$0.167139 \mathrm{E}-0$

$0.149053 E=0$

$0.215478 \mathrm{E}-03$

$0.412166 E-0$

$0.252569 \mathrm{E}-05$

$0.669975 E \quad 00$ $0.169690 E=01$ $0.150941 E-02$ $0.216913 E-03$ $0.411686 \mathrm{E}=0$ $0.942574 E-05$ $0.247603 E-05$ $0.706935 \mathrm{E}-02$ $0.497904 E-03$ $0.597770 E=04$ $0.978755 \mathrm{E}-05$ $0.197610 E-05$
$0.404267 E-00$ $0.465166 E-06$
$0.333553 E=00$ $0.756074 E-02$ $0.755215 \mathrm{E}-03$ $0.126943 \mathrm{E}-03$ $0.285036 E-04$ $0.774726 \mathrm{E}-05$ $0.241700 E-05$

$0.383056 E-00$ $0.104595 \mathrm{E}-01$ $0.119390 E-02$ $0.222737 \mathrm{E}-03$ $0.544688 E-04$ $0.159122 E-04$ $0.528451 E-05$

$0.460590 E-00$ $0.161098 E-01$ $0.220652 E-02$ $0.476487 E-03$ $0.131682 E-03$ $0.427361 E-04$ $0.155664 E-04$

$0.591925 E 00$ $0.292770 E=01$ $0.524067 E-02$ $0.141508 \mathrm{E}=02$ $0.474291 \mathrm{E}-03$ $0.182493 E-03$ $0.774461 E-04$

$0.249416 E-00$ $0.297265 E-02$ $0.173260 \mathrm{E}-03$ $0.185793 E-04$ $0.283690 E=05$ $0.126494 E-06$

$0.265546 E-00$ $0.458675 E-02$ $0.381105 E=03$ $0.553828 \mathrm{E}-04$ $0.110013 E-04$ $0.268762 E-05$ $0.762668 E=06$

$0.311804 E-00$ $0.670000 E-02$ $0.651216 E-03$ $0.107011 \mathrm{E}-03$ $0.235091 E-04$ $0.625337 E=05$ $0.190997 E-05$

$0.387016 E=00$ $0.111236 E-01$ $0.133540 E-02$ $0.260109 E-03$ $0.658744 E-04$ $0.198007 E-04$ $0.673228 E-05$

0.521688 E 00 $0.224217 E-01$ $0.365803 E-02$ $0.918362 E-03$ $0.289414 E-03$ $0.105479 E-03$ $0.426287 E-04$

$0.190304 E-00$ $0.166282 E-02$ $0.779974 \mathrm{E}-04$ $0.702894 E-05$ $0.927317 E-06$ $0.158273 E-06$ $0.326097 E-07$

$0.278414 E-00$ $0.349544 E-02$ $0.201378 E-03$ $0.20=531 E-04$ $0.3 r 4015 E-05$ $0.558605 E-06$ $0.121603 E-06$

$0.782183 E-00$ $0.354274 \mathrm{E}=02$ $0.201583 \mathrm{E}-03$ $0.205406 \mathrm{E}-04$ $0.294210 \mathrm{E}-05$ $0.530688 E-06$ $0.113361 \mathrm{E}-06$

$0.215735 E-00$ $0.205763 E-02$ $0.979357 E-04$ $0.869410 E-05$ $0.111250 E-05$ $0.182480 E-06$ $0.35922 P E=07$

$0.219080 E-00$ $0.209962 E-02$ C.990235E-04 $0.867089 E-05$ $0.109216 E-05$ $0.176141 E=06$ 0.340708 E-07

$0.289673 E=00$ $0.372680 \mathrm{E}-02$ $0.213928 E-03$ $0.218430 E-04$ $0.312321 E=05$ $0.561018 \mathrm{E}-06$ $0.119145 E-06$

$0.220853 E-00$ $0.301111 E-02$ $0.211923 E-03$ $0.269583 \mathrm{E}-04$ $0.478198 E-05$ 
Toble 2. Spotiol Moments, Plone Isotropic Source-Continued

n

$p=-1 / 2$

$1 / 2$

$3 / 2$

$5 / 2$

$7 / 2$

$9 / 2$

$11 / 2$

$M E V=0.40$

$2=29$.

$\ell=0$

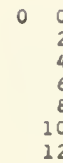

0

$\begin{array}{rr}0 & 0.182812 E-01 \\ 2 & 0.817533 E-01 \\ 4 & 0.103283 E-01 \\ 6 & 0.193513 E-02 \\ 8 & 0.455413 E-03 \\ 10 & 0.125019 E-03 \\ 12 & 0.384329 E-04\end{array}$

$0.678134 E 00$

$0.176946 E-01$

$0.160539 E-02$

$0.233984 \mathrm{E}-03$

$0.448806 E-04$

$0.273872 \mathrm{E}-05$ $0.137934 E-04$
$0.103596 \mathrm{E}-04$
$M E V=1.00$

$M E V=0.70$ $z=29$.

\begin{abstract}
$0.188548 E$ OI
$0.813055 \mathrm{E}-01$

$0.996169 E-02$

$0.181938 \mathrm{E}-02$

$0.418844 E-03$

$0.112761 \mathrm{E}-03$

$0.340616 E-04$
\end{abstract}

$0.703219 E \quad 00$ $0.198668 E-02$ $0.307621 E-03$ $0.620579 E-04$ $0.149609 E-04$ $0.410960 E-05$
$0.414906 E-00$ $0.755310 E-02$ $0.546031 E-03$ $0.667792 E-04$ $0.110864 E-04$ $0.226239 E-05$

$0.537052 E-06$

$0.431365 E-00$ $0.635590 \mathrm{E}-03$ $0.806377 E-04$ $0.137934 E-04$ $0.288673 E-05$
$0.700382 E-06$
$0.299613 E-00$ $0.404847 E-02$ $0.240007 E-03$ $0.250794 E-04$ $0.364917 E-05$ $0.142652 E-06$

$0.315182 E-00$ $0.288856 E-03$ $0.315300 E-0$ $0.475303 E-05$ $0.891563 E-06$ $0.196351 E-06$

0.331791 E-00 $0.523912 \mathrm{E}-02$ $0.347898 E-03$ $0.397076 E-04$ $0.620897 \mathrm{E}-05$ $0.271824 E-06$

$M E V=2.00$ $2=29$.

$0.167823 E \quad 01$ $0.835772 E-01$ $0.115887 E-01$ $0.234902 E-02$ $0.592132 E-03$ $0.172851 E-03$ $0.561913 E-04$
$0.448657 \mathrm{E}-00$ $0.930545 E-0$ $0.739939 E-0$ $0.171958 E-04$ $0.369509 E-0$ $0.664481 E-06$ $0.917469 E-06$ $0.838471 E-02$ $0.460502 E-02$ $0.120140 E-05$

. $234665 \mathrm{E}-00$ $0.245524 E-02$ $0.121948 E-03$ $0.110434 E-0$ $0.142278 E-05$ $0.232963 E-06$ $0.455052 E-07$

$0.248745 E-00$ $0.284356 E-02$ $0.150668 E-03$ $0.143479 E-04$ $0.143479 E-04$ $0.326288 E-06$ $0.656365 E-07$

$0.263926 E-00$ $0.186389 E-03$

$0.186852 E-04$ 0. $61580 E-05$ $0.459370 E-06$ $0.953161 E-07$

. $311274 E-00$ . $497948 E-02$ $0.339622 E-03$ $0.395653 E-04$ $0.627919 E-05$ $0.122828 E-05$ $0.280184 E-06$

$0.390316 E-00$ $0.874185 E-02$ $0.772257 \mathrm{E}-03$ $0.111142 \mathrm{E}-03$ $0.211032 E-04$ $0.482620 E-05$ $0.482620 E-05$

$0.862994 E-03$

$0.275568 E-03$ . $322005 E-01$ $0.812827 \mathrm{E}-03$

$0.199720 E-03$

$0.572869 \mathrm{E}-04$

$0.183979 E-04$
$0.577916 E 00$ $0.185875 \mathrm{E}-01$ $0.360649 \mathrm{E}-03$ $0.211766 \mathrm{E}-04$ $0.630392 E-05$ $0.329553 E-02$ $0.207707 \mathrm{E}-02$ $2=29$.

0.128370E O1 $0.920964 E-01$ $0.180872 E-01$ $0.496602 E-02$ $0.163932 \mathrm{E}-02$ $0.610969 E-03$ $0.248663 E-03$

$\begin{array}{ll}0.860967 E-00 & 0.700630 E 00 \\ 0.474054 E-01 & 0.326040 E-01 \\ 0.799009 E-02 & 0.495631 E-02 \\ 0.195283 E-02 & 0.111742 E-02 \\ 0.585341 E-03 & 0.312812 E-03 \\ 0.200692 E-03 & 0.100997 E-03 \\ 0.758668 E-04 & 0.361751 E-04\end{array}$
MEV $=0.05$ $z=50$.

$0.211954 \mathrm{E} 01$ $0.657000 E-01$ $0.610643 E-02$ $0.882671 E-03$ $0.165809 E-03$ $0.372759 E-04$
$0.957510 E-05$

$0.643694 E$ OO $0.123535 E-01$ $0.870923 E-03$ $0.102742 E-\sigma_{3}$ $0.164108 E=04$ $0.322182 E-05$ $0.736462 E-06$

$$
M E V=0.10
$$

$2=50$.

$\begin{array}{llll}0 & 0 & 0.206696 E & 01\end{array}$ $0.588604 E-01$ $0.503548 E-02$ $0.671967 \mathrm{E}-03$ $0.116840 E-03$ $0.243686 E-04$ $0.581877 E-05$
$0.653575 E \quad 00$ $0.117894 E-01$ $0.772687 \mathrm{E}-03$ $0.846850 E-04$ $0.125775 E-04$ $0.229882 E-05$ $0.409851 E-06$
$0.371104 E-00$ $0.484113 E-02$ $0.267379 E-03$ $0.261691 E-04$ $0.358895 E-05$ $0.619234 E-06$ $0.126524 E-06$

$0.383376 E-00$ $0.480885 E-02$ $0.249928 E-03$ $0.229124 E-04$ $0.293975 E-05$ $0.474477 \mathrm{E}-06$ $0.907249 E-07$
$0.463916 E-00$ $0.122910 E-0$ $0.121260 E-02$ $0.190685 \mathrm{E}-0$ $0.390628 E-04$ $0.955434 E-05$ $0.266063 E-05$

$0.602247 E 00$ $0.244578 E-01$ $0.341245 E-02$ $0.719047 E-03$ $0.190020 E-03$ $0.583044 E-04$ $0.199466 E-04$

$0.259326 E-00$ $0.245494 E-02$ $0.109232 \mathrm{E}-03$ $0.901196 E-05$ $0.107203 E-05$ $0.163696 E-06$ $0.300484 E-07$

$0.27 .0443 E-00$ $0.250900 E-02$ $0.828367 \mathrm{E}-05$ $0.229879 E-07$ $0.106290 \mathrm{E}-0 \mathrm{~S}$ $0.927912 E-06$ $0.133241 E-06$

$0.192933 E-00$ $0.161240 E-02$ $0.682331 E-04$ $0.541726 E-05$ $0.623271 E-06$ $0.923745 E-07$ $0.165039 E-07$

$0.205605 E-00$ $0.189376 E-02$ $0.861210 E-04$ $0.723496 E-05$ $0.871723 E-06$ $0.134307 E-06$ $0.248086 E-07$

$0.219377 \mathrm{E}-00$ . $222680 E-02$ $0.108897 \mathrm{E}-03$ $0.969129 E-05$ $0.122455 \mathrm{E}-05$ $0.196437 E-06$ $0.375769 \mathrm{E}-07$

$0.263030 E-00$ $0.350465 E-02$ $0.210789 E-03$

$0.221668 E-04$ $0.322103 E-05$ $0.582725 E-06$ $0.123884 E-06$

$0.338120 E-00$ $0.651889 \mathrm{E}-02$ $0.520865 E-03$ $0.691255 E-04$ $0.122406 E-04$ $0.263112 E-05$ $0.651950 E-06$

$0.532648 E \quad 00$ $0.192215 E-01$ $0.248629 E-02$ $0.493373 E-03$ $0.123859 E-03$ $0.363136 E-04$ $0.119233 E-04$

$0.198934 E-00$ $0.143179 E-02$ $0.525039 E-04$ $0.370317 E-05$ 
Table 2. Spotial Moments, Plone Isotropic Source - Continued

$\begin{array}{lllllll}p=-1 / 2 & 1 / 2 & 3 / 2 & 5 / 2 & 1 / 2 & 9 / 2 & 11 / 2\end{array}$

\section{MEV $=0.20 \quad Z=50$.}

0.201897 E OI 0.502898 E 00 $0.551487 E-01$ $0.452350 \mathrm{E}-02$ $0.579002 E-03$ C. $966167 \mathrm{E}-04$ $0.153492 E-04$
$0.443893 E-03$ $\begin{array}{ll}0.502898 E & 00 \\ 0.116695 E-01\end{array}$ $0.116695 E-01$ $0.780883 \mathrm{E}-04$ C. $111701 \mathrm{E}-04$ $0.190575 E=05$

$0.403319 E-06$

$M E V=0.40$ $z=50$.

0
$0.196068 E 01$ $0.536107 E-0$ : $0.438571 E-02$ C.55835IE-0 C. $924943 E-04$ C. $183638 E-04$ $C .183638 E-04$
$0.417223 E-05$
$0.674639 E 00$ $0.120860 E-01$ $0.769632 \mathrm{E}-03$ C. $012866 \mathrm{E}-04$ . $15844 E-04$ O. $115844 E-04$ $0.202650 E-05$
$C .412643 E-06$
$0.410328 E-00$ C. 529260 E-02 $0.272686 E=03$ $0.244298 E-04$ $0.303927 E-05$ $0.473365 E-06$ $0.473365 E-06$
$0.870680 E-07$ $M E V=0.70$
$z=50$.

$0.395172 E-00$ $0.492287 E-02$ C. $249685 E-03$ $0.222154 E-04$ $0.275912 E-05$ $0.430482 \mathrm{E}-06$ C. 795103 E-07

0

$0.188653 E$ OI $0.534659 E-01$ C. $450472 E-02$ $0.587444 E-03$ $0.992870 \bar{E}=04$ $0.200526 E-04$ $0.462383 E-05$

$0.690286 E 00$ $0.130582 E-01$ $0.864804 E-03$ $0.941435 \mathrm{E}-04$ $0.137491 E-04$ $0.245479 E-05$ $0.508623 E-06$ $M E V=1.00$ $z=50$.

0 $0.182016 E$ OI $0.530072 E-01$ $0.469954 E-02$ C. $631781 \mathrm{E}-03$ $0.109639 \mathrm{E}-03$ $0.226675 E=04$ $0.533797 \mathrm{E}-05$ $M E V=2.00$

$0.705028 \mathrm{E} 00$ $0.141435 E-0 I$ $0.979837 \mathrm{E}-03$ $0.110616 E-03$ $0.166582 \mathrm{E}-04$ $0.166582 E-04$
$C .305452 E-05$ $0.648015 E-06$

$0.431057 \mathrm{E}-00$ $0.598760 E-02$ $0.324840 E-03$ $0.302626 E-04$ $0.388414 E-05$ $0.620705 E-06$ $0.116672 E-06$

$0.451152 E-00$ $0.676105 E-02$ C. $388001 E-03$ $0.377907 \mathrm{E}-04$ $0.503272 E-05$ $0.503272 E=05$
$0.830089 E=06$ $0.160413 E-06$ $Z=50$.

0

$0.166229 E$ O1 $0.563596 E-01$ $0.554613 E-02$ $0.826278 E-03$ $0.157029 E=03$ $0.894329 E-05$

$0.743289 \mathrm{E} 00$ $0.177901 \mathrm{E}-01$ $0.142079 E-02$ $0.180549 E-03$ $0.301202 E-04$ 0010405 $0.139298 E-05$ $M E V=4.00$ $z=50$.

0 $0.147129 E 01$ $0.604356 E-01$ $0.704963 \mathrm{E}-02$ $0.121655 E-02$
$0.263396 E-03$ $0.665068 E=04$

$0.505939 E \quad 00$ $0.941511 E-02$ $0.639671 \mathrm{E}-03$ $0.715387 \mathrm{E}=04$ $0.107200 E=04$ $0.196107 E=05$ $0.415794 E-06$

0.797057 E 00 $0.244225 E-01$ $0.239096 E-02$ $0.360559 \mathrm{E}-03$ $0.360559 E-03$ $0.698191 E-04$
$0.160149 E-04$ $0.416192 E-05$

$0.589712 E \quad 00$ $0.147884 E-01$ $0.127496 E-02$ $0.173800 \mathrm{E}-03$ $0.308944 E-04$ $0.657447 \mathrm{E}-05$ $0.159766 E-05$ MEV $=10.00$

$z=50$.

0

$0.125340 E$ OI $0.691817 \mathrm{E}-0 \mathrm{I}$ $0.106504 \mathrm{E}-0 \mathrm{~J}$ $0.234724 E-02$ $0.633232 E-03$

$0.195631 \mathrm{E}-03$ $M E V=0.10$

$c$

$0.215598 E 01$ 0.41434 EE-OI $0.248478 E-02$ C. $240327 \mathrm{E}-03$ C. 311030 E-04 C. $493435 E-05$ C. $912627 E=06$ $0.872687 E$ OO
$0.38958 \& E-0 I$ $0.529093 E-02$ $0.105830 \varepsilon-02$ $0.263255 E-03$ $0.758088 E-04$ $0.243809 E-04$ $z=82$.

$0.722447 E 00$ $0.281765 E-01$ $0.352000 E-02$ $0.659050 E-03$ $0.154970 E-03$ $0.424,738 E-04$ $0.130781 \mathrm{E}-04$

$0.637042 E 00$ $0.782347 \bar{E}-02$ $0.361457 \mathrm{E}-03$ C. $288851 E-04$ $0.321144 E-05$ 0.44808 $0.448808 E-06$
$0.744238 E-07$
$0.281254 E-00$ $0.262969 E-02$ $0.109743 \mathrm{E}=03$ C. $835840 \mathrm{E}-05$ $0.911258 \mathrm{E}-06$ $0.127045 \mathrm{E}-06$ $0.212496 \varepsilon=07$

$0.295324 E-00$ $0.290048 \mathrm{E}-02$ $0.124176 E-03$ $0.959416 E-05$ $0.105390 E-05$ $0.147373 E-06$ $0.246440 E-07$

$0.314890 E-00$ $0.338557 E-02$ $0.154425 E-03$ $0.125194 \mathrm{E}-04$ $0.142900 E-05$ $0.208232 E-06$ $0.354156 \varepsilon-07$

$0.334208 E-00$ $0.393286 E-02$ $0.191744 E-03$ $0.163844 E-04$ $0.195318 E-05$ $0.292508 E-06$ $0.518768 E=07$

$0.388619 E-00$ $0.587233 \mathrm{E}-02$ $0.347359 E-03$ $0.347490 E-04$ $0.473557 \mathrm{E}-05$ $0.797090 E-06$ $0.156869 E-06$

$0.476666 E-00$ $0.101343 E-01$ $0.784646 \mathrm{E}-03$ $0.980988 \mathrm{E}-04$ $0.161936 E-04$ $0.322799 E-05$ $0.739569 E-06$

$0.628176 E 00$ $0.219391 E-01$ $0.255667 E-02$ $0.452881 E-03$ $0.101557 E-03$ $0.266945 E-04$ $0.792272 E-05$

$0.252034 \mathrm{E}-00$ $0.160132 \mathrm{E}-02$ $0.478044 E-04$ $0.271958 E-05$ C.228559E=06 (1) C. $338876 E-08$

$0.218255 \varepsilon-00$ $0.159510 E-02$ $0.559286 E-04$ $0.369575 E-05$ $0.357022 E-06$ $0.447867 E-07$ $0.681999 \varepsilon=08$

$0.230813 E-00$ $0.179301 E-02$ $0.650711 E-04$ $0.439302 E-05$ $0.430089 E-06$ $0.543780 \mathrm{E}-07$ $0.831279 \varepsilon-08$

$0.248479 E-00$ $0.214173 E=02$ $0.836886 E-04$ $0.597950 E-05$ $0.612625 E-06$ $0.804179 E-07$ $0.126889 E=07$

$0.266149 E=00$ $0.254087 E-02$ $0.107121 E-03$ $0.812906 E-05$ $0.875396 E-O S$ $0.119888 \mathrm{E}-06$ $0.196272 \mathrm{E}=07$

$0.317090 E-00$ $0.399784 \mathrm{E}-02$ $0.209028 E-03$ $0.189103 E-04$ $0.236377 \mathrm{E}-05$ $0.368629 E-06$ $0.677315 E-07$

$0.403018 E-00$ $0.741759 E-02$ $0.521921 E-03$ $0.603830 E=04$ $0.932378 E-05$ $0.175164 E-05$ $0.380376 E=06$

$0.560420 E$ OO $0.177696 E-01$ $0.194688 \mathrm{E}-02$ $0.328258 E-03$ $0.705565 E-04$ $0.178652 E-04$ 
Toble 2. Spotiol Moments, Plone Isotropic Source - Continued

n

$$
p=-1 / 2
$$

$1 / 2$

$3 / 2$

$5 / 2$

$7 / 2$

$9 / 2$

$11 / 2$

$\boldsymbol{l}=0 \begin{array}{r}0 \\ 2 \\ 4 \\ 6 \\ 0 \\ 1 Q \\ 12\end{array}$

0

0
2
4
6
8
10
12

$Z=82$.

$0.208503 E 01$

$0.357941 E-01$

$0.192109 E-02$

$0.166777 E-03$

$0.194256 E-04$

$0.278030 E-05$

$0.464950 E-06$

MEV $=0.40$

$0.650141 E 00$ $0.731934 \mathrm{E}-02$ $0.306302 E-03$ $0.221426 E-04$ $0.222825 E-05$ $0.282187 \mathrm{E}-06$ $0.282187 E-06$
$0.424621 E-07$

$0.379085 E-00$ $0.305595 E-02$ $0.102961 E-03$ $0.629961 E-05$ $0.553039 E-06$ $0.623668 E=07$ $0.848229 E-08$

$Z=82$ 。

$0.200135 E$ OI

$0.328401 E-01$

$0.168246 E-02$

$0.139326 E-03$

$0.154738 E-04$

$0.211138 E-05$

$0.336599 E-06$

$0.666398 \mathrm{E} \quad 00$ $0.735518 \mathrm{E}-02$ $0.297491 E-03$ $0.206778 \mathrm{E}-04$ $0.199540 E-05$

$0.241952 E-06$

$0.348270 E-07$

MiEV $=0.70$

$z=82$.

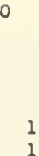

$0.190429 E \quad 01$

$0.317921 E-01$

$0.164978 \mathrm{E}-02$

$0.137813 \mathrm{E}-03$

$0.153900 E-04$

$0.210611 E-05$

$0.336048 E-06$

$$
M E V=1.00
$$

$0.686462 E \quad 00$

$0.791503 E-02$

$0.328727 \mathrm{E}-03$

$0.232506 E-04$

$0.227000 E-05$

$0.277345 E-06$

$0.401029 E-07$

$0.399654 E-00$ $0.324162 \mathrm{E}-02$ $0.107209 E-03$ $0.637633 \mathrm{E}-05$ $0.541229 E-06$ $0.588189 E-07$ $0.769281 E-08$

$0.425935 E-00$ $0.370649 E-02$ $0.127998 \mathrm{E}-03$ $0.783967 \mathrm{E}-05$ $0.679365 E-06$ $0.749275 \mathrm{E}-07$ $0.990174 E-08$

$0.181865 E$ OL $0.315889 E-01$

$0.169680 E-02$ $0.145990 E-03$ $0.167264 E-04$ $0.234107 \mathrm{E}-0.5$ $0.381059 E-06$ $2 \approx 82$.

$0.705370 E \quad 00$ $0.065881 E-02$ $0.376899 E-03$ $0.276802 E-04$ $0.278880 E-05$ $0.350034 E-06$ $0.518154 E-07$

$0.451625 E-00$ $0.427977 E=02$ $0.157176 E-03$ $0.101026 E-04$ $0.910832 E=06$ $0.103876 E-06$ $0.141294 \mathrm{E}-07$ MEV $=2.00$

$2=82$.

$0.165215 E$ OI $0.337757 E-01$ $0.210098 \mathrm{E}-02$ $0.206011 E-03$ $0.265658 E=04$ $0.414345 E-05$ $0.745462 E-06$

$0.745919 \mathrm{E} \quad 00$ $0.112530 E-01$ $0.581245 E-03$ $0.494502 E-04$ $0.567501 E-05$ $0.801165 E-06$ $0.132073 E-06$

$0.509848 \mathrm{E} \quad 00$ $0.618643 E-02$ $0.277055 E-03$ $0.210500 E-04$ $0.219634 E-05$ $0.2854 \mathrm{C6E}-06$ $0.437048 E-07$ $M E V=4.00$ $z=82$.

$0.144942 \mathrm{E} \quad 01$ $0.376061 E-01$ $0.288659 E-02$ $0.340194 E-03$ $0.517003 E-04$ $0.936000 E-05$ $0.193120 E-05$

\begin{abstract}
$0.803859 E \quad 00$ $0.163267 E-01$ $0.107738 \mathrm{E}-02$ $0.112925 E-03$ $0.155679 E-04$ $0.259106 E-05$ $0.496270 E-06$
\end{abstract}

MEV $=0.025$ Air

0 $2 \quad 0.201025 E 01$ $0.479180 E-01$ $0.165278 \mathrm{E}-01$ $0.679527 \mathrm{E}-02$ $0.313094 E-02$ $0.156476 E-02$ MEV $=0.05$

$0.664624 E \quad 00$ $0.332702 E-01$ $0.589477 \mathrm{E}-02$ $0.159400 E-02$ $0.541449 E-03$ $0.213050 E-03$ $0.930497 E-04$

Air

$0.196193 E$ OI $0.186748 E-00$ $0.464103 E-01$ $0.159093 E-01$ $0.649552 E=02$ C. $297072 \mathrm{E}-02$ $0.147334 E-02$

$0.674383 E 00$ $0.341924 E-01$ $0.607313 E-02$

$0.163909 E-02$

$0.554481 E-03$

$0.217016 E-03$

$0.942065 E-04$
$0.600905 E 00$ $0.103370 E-01$ $0.612198 \mathrm{E}-03$ $0.588505 E-04$ $0.753816 E-05$ $0.117620 E=05$ $0.212607 E-06$

$0.397379 E-00$ $0.122010 \mathrm{E}-01$ $0.161166 E-02$ $0.350845 E-03$ $0.100143 E-03$ $0.340512 \mathrm{E}-04$ $0.131104 E-04$

$0.409994 E-00$ $0.129702 E-01$ $0.173417 \mathrm{E}-02$ $0.379187 E-03$ $0.108275 E-03$ $0.367447 E-04$ $0.140989 E-04$
$0.266540 E-00$ $0.163054 E-02$ $0.453997 E-04$ $0.238635 E-05$ $0.184513 E-06$ $0.186514 E-07$ $0.230386 E-08$

$0.285394 E-00$ $0.179503 E-02$ $0.497892 \mathrm{E}-04$ $0.257265 E-05$ $0.194040 E-06$ $0.190388 \mathrm{E}-07$ $0.227507 E-08$

$0.310020 E-00$ $0.213839 \mathrm{E}-02$ $0.629108 E-04$ $0.338812 \mathrm{E}-05$ $0.263410 E-06$ $0.264360 E-07$

$0.321299 E-08$

$0.334668 E-00$ $0.256099 E-02$ $0.812126 E-04$ $0.463831 E-05$ $0.378356 E-06$ $0.395406 E-07$ $0.497580 E-08$

$0.392598 E-00$ $0.397881 \mathrm{E}=0$ $0.157715 E-03$ $0.108571 E-0$ $0.104140 E-05$ $0.125678 \mathrm{E}-06$ $0.180105 E-07$

$0.488873 E-00$ $0.733190 \mathrm{E}-02$ $0.396305 E-03$ $0.353791 \mathrm{E}-04$ $0.425300 E-05$ $0.627381 E-06$ $0.107804 E-06$

$0.283291 E-00$ $0.587097 \mathrm{E}-0$ $0.600080 E-03$ $0.107433 E-03$ $0.261361 E=04$ $0.775836 \mathrm{E}-05$ $0.265363 \mathrm{E}-05$

$0.295011 \mathrm{E}-00$ $0.638905 E-02$ $0.667327 \mathrm{E}-03$ $0.120819 E-03$ $0.295539 E-04$ $0.879046 \mathrm{E}-0 \mathrm{E}$ $0.300584 E-05$

$0.205256 E-00$ $0.991963 E-03$ $0.233028 E-04$ $0.106586 E-05$ $0.732142 E-07$ $0.667502 E-08$ $0.752316 E-09$

$0.221937 E-00$ $0.112254 E-02$ $0.266201 E-04$ $0.120976 E-05$ $0.817767 E-07$ $0.729033 E-08$ $0.799866 \mathrm{E}-09$

$0.244060 E-00$ $0.137824 E-02$ $0.351542 E-04$ $0.168422 E-05$ $0.118464 E-06$ $0.108885 E-07$ $0.122333 E-08$

$0.266572 E-00$ $0.169580 E-02$ $0.471855 E-04$ $0.242100 E-05$ 
Table 2. Spatial Moments, Plane Isotropic Source - Continued

Air

$M E V=0.10$

$\begin{array}{ll}0.197775 E-01 & 0.671151 E 00 \\ 0.191636 E-00 & 0.343062 E-01 \\ 0.482065 E-01 & 0.614250 E-02 \\ 0.166754 E-01 & 0.166879 E-02 \\ 0.685712 E-02 & 0.567631 E-03 \\ 0.315453 E-02 & 0.223191 E-03 \\ 0.157217 E-02 & 0.972705 E-04\end{array}$

$$
\text { MEV }=0.20
$$

Air

0

\begin{tabular}{|c|c|}
\hline $\begin{array}{l}0.196606 \mathrm{E} 01 \\
0.192497 \mathrm{E}-00 \\
0.487461 \mathrm{E}-01 \\
0.169338 \mathrm{E}-01 \\
0.698299 \mathrm{E}-02 \\
0.321832 \mathrm{E}-02 \\
0.160583 \mathrm{E}-02\end{array}$ & $\begin{array}{l}0.673536 E-00 \\
0.348191 E-01 \\
0.627951 E=02 \\
0.171391 E=02 \\
0.584757 E=03 \\
0.230389 E-03 \\
0.100537 E-03\end{array}$ \\
\hline
\end{tabular}

MEV $=0.40$

Air

$\begin{array}{ll}0.193466 E ~ 01 & 0.680035 E 00 \\ 0.192145 E-00 & 0.358811 E-01 \\ 0.492124 E-01 & 0.656434 E-02 \\ 0.172423 E-01 & 0.181043 E-02 \\ 0.715799 E-02 & 0.622651 E-03 \\ 0.331694 E-02 & 0.246891 E-03 \\ 0.166251 E-02 & 0.108304 E-03\end{array}$

$$
M E V=0.70
$$

Air

0

$\begin{array}{ll}0.193466 E ~ 01 & 0.680035 E 00 \\ 0.192922 E-00 & 0.359868 E-01 \\ 0.494984 E-01 & 0.659264 E-02 \\ 0.173535 E-01 & 0.181893 E-02 \\ 0.720420 E-02 & 0.625464 E-03 \\ 0.333708 E-02 & 0.247877 E-03 \\ 0.167151 E-02 & 0.108654 E-03\end{array}$

$$
M E V=1.00
$$

Air

0

$\begin{array}{ll}0.182248 E ~ 01 & 0.704500 E 00 \\ 0.186279 E-00 & 0.394339 E-01 \\ 0.491333 E-01 & 0.753406 E-02 \\ 0.176428 E-01 & 0.214872 E-02 \\ 0.747898 E-02 & 0.759373 E-03 \\ 0.352943 E-02 & 0.308074 E-03 \\ 0.179791 E-02 & 0.137844 E-03\end{array}$

$$
\text { MEV }=2.00
$$

Air

0

$\begin{array}{ll}0.166734 \mathrm{E} 01 & 0.741988 \mathrm{E} \quad 00 \\ 0.175920 \mathrm{E}-00 & 0.451362 \mathrm{E}-01 \\ 0.482984 \mathrm{E}-01 & 0.919118 \mathrm{E}-02 \\ 0.179694 \mathrm{E}-01 & 0.275856 \mathrm{E}-02 \\ 0.785902 \mathrm{E}-02 & 0.101717 \mathrm{E}-02 \\ 0.381371 \mathrm{E}-02 & 0.427968 \mathrm{E}-03 \\ 0.199251 \mathrm{E}-02 & 0.197714 \mathrm{E}-03\end{array}$

0 $0.161451 E-00$ $0.467705 E-02$ $0.182931 E-01$ $0.836919 \mathrm{E}-02$ $0.423029 \mathrm{E}=02$ $0.229413 E-02$

$0.794306 E$ OO $0.540810 E-01$ $0.120556 \mathrm{E}-01$ $0.389930 E-02$ $0.153215 E-02$ $0.681277 E-03$ $0.330549 E-03$ $M E V=10.00$

Air

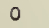

$0.124042 E \quad 01$ $0.137570 E-00$ $0.427443 E-01$ $0.179803 E-01$ $0.881183 E-02$ $0.475029 \mathrm{E}-02$ $0.274325 E-02$

$$
3 / 2
$$$$
5 / 2
$$$$
7 / 2
$$

$0.405790 E-00$ $0.128361 E-01$ $0.172244 E-02$ $0.378026 E-03$ - $108312 E-03$ $0.368695 E-04$ $0.141852 \mathrm{E}-04$

$0.408889 E-00$ $0.131132 E-01$ $0.177502 E-02$ $0.391767 E-03$ $0.112674 E-03$ $0.384525 E-04$ $0.148196 E-04$

$0.417408 E-00$ $0.137837 E=01$ $0.190210 E-02$ $0.425645 E-03$ $0.123704 E-03$ $0.425664 E=04$ $0.165154 \mathrm{E}-04$

$0.417408 E=00$ $0.138157 E-01$ $0.190863 E-02$ $0.427196 E-03$ $0.124116 E-03$ $0.426815 \mathrm{E}-04$ $0.165461 E-04$

$0.450422 E-00$ $0.163580 E-01$ $0.240526 E-02$ $0.564296 E-03$ $0.170240 E-03$ $0.604069 \mathrm{E}-04$ $0.240552 E-04$

$0.504011 E 00$ $0.210061 E-01$ $0.339463 \mathrm{E}-02$ $0.856301 \mathrm{E}-0$ $0.273960 \mathrm{E}-03$ $0.102121 E-03$ $0.424296 \mathrm{E}-04$

$0.585224 E \quad 00$ $0.293733 \mathrm{E}-01$ $0.541884 E-02$ $0.151819 E-02$ $0.529941 E-03$ $0.212814 \mathrm{E}-03$ $0.943643 E-04$

$0.732230 \mathrm{E}$ OO $0.488896 \mathrm{E}-01$ $0.111437 \mathrm{E}-01$ $0.371383 E-02$ $0.150375 \mathrm{E}-02$ $0.688493 E-03$ $0.345134 E-03$
$0.291090 E-00$ $0.626377 \mathrm{E}-02$ $0.653941 E-03$ $0.128499 \mathrm{E}-03$ $0.290232 \mathrm{E}=04$ $0.864445 E-05$ $0.864445 E-05$
$0.295992 E-05$

$0.293980 E-00$ $0.642847 \mathrm{E}-02$ $0.678131 E-03$ $0.123727 \mathrm{E}-03$ $0.304464 \mathrm{E}-04$ $0.909816 \mathrm{E}-05$ $0.312244 E-05$

$0.301965 E-00$ $0.685147 \mathrm{E}-02$ $0.740609 \mathrm{E}-03$ $0.137517 \mathrm{E}-03$ $0.342930 \mathrm{E}-04$ $0.103558 E-04$ $0.358449 E-05$

$0.301965 E-00$ $0.686464 \mathrm{E}-02$ $0.742722 \mathrm{E}-03$ $0.137922 \mathrm{E}-03$ 0.343801 E-0 4 $0.103747 \mathrm{E}-04$ $0.358775 E-05$

$0.333501 E-00$ $0.857957 \mathrm{E}-02$ $0.100805 E-02$ $0.199120 \mathrm{E}-03$ $0.521291 E-04$ $0.163798 \mathrm{E}-04$ $0.586199 \mathrm{E}-05$

$0.386662 E-00$ $0.119437 \mathrm{E}-01$ $0.158825 E-02$ $0.344746 \mathrm{E}-03$ $0.973710 \mathrm{E}-0$ $0.325942 E-04$ $0.123135 E-04$

0.471801 E-00 $0.186297 \mathrm{E}-0$ $0.293889 \mathrm{E}-\mathrm{O} 2$ $0.729354 \mathrm{E}-03$ $0.230075 E-03$ $0.846097 \mathrm{E}-04$ $0.346875 E-04$

$0.639936 E$ OO $0.368079 E-01$ $0.760442 \mathrm{E}-02$ $0.234723 E-02$ $0.891152 \mathrm{E}-03$ $0.385811 \mathrm{E}-03$ $0.184204 \mathrm{E}-0_{3}$
$0.227021 E-00$ $0.353951 E-02$ $0.295843 E-03$ $0.450466 \mathrm{E}-04$ $0.954706 E-05$ $0.251074 E=05$ $0.770494 E-06$

$0.229607 E-00$ $0.364509 E-02$ $0.308336 \mathrm{E}-03$ $0.473315 E-04$ $0.100886 E-04$ $0.266403 E-05$ $0.819959 E-06$

$0.236783 E-00$ $0.392527 E-02$ $0.341880 E-03$ $0.536086 E-04$ $0116141 \mathrm{E}-04$ $0.116141 E-04$ $0.310674 E-05$
$0.966338 E-06$

$0.236783 E-00$ $0.393170 E=02$ $0.342714 E-03$ $0.537399 E-04$ $0.116370 E-04$ $0.311044 E-05$ $0.966550 E-06$

$0.265498 E-00$ $0.511664 E-02$ $0.493388 E-03$ $0.834817 E-04$ $0.192050 E=04$ $0.539574 E-05$ $0.174894 \mathrm{E}-05$

$0.315229 E-00$ $0.757135 E=02$ $0.848698 \mathrm{E}-03$ $0.161113 E-03$ $0.406685 E-04$ $0.123455 E-04$ $0.427517 \mathrm{E}-05$

$0.398161 E-00$ $0.128445 E-01$ $0.176809 E-02$ $0.394250 E-03$ $0.113651 E-03$ $0.386278 \mathrm{E}-04$ $0.147572 E-04$

$0.573129 E \quad O 0$ $0.290469 \mathrm{E}-01$ $0.551207 \mathrm{E}=02$ $0.159134 \mathrm{E}-02$ $0.571044 \mathrm{E}-03$ $0.235381 E-03$ $0.107696 \mathrm{E}-03$

$0.186095 E-00$ $0.219937 E-02$ $0.150545 E-03$ $0.195426 \mathrm{E}-04$ D. $162126 E-O 5$ $0.847495 \mathrm{E}-06$ $0.234534 E-06$

$0.188406 \mathrm{E}-00$ $0.227104 E-02$ $0.157556 \mathrm{E}-03$ $0.206449 E-04$ $0.385129 E-05$ $0.905783 E-06$ $0.251586 \mathrm{E}-06$

$0.194833 E-00$ $0.246530 E-02$ $0.176876 E-03$ $0.237593 E-04$ $0.451833 E-05$ $0.107913 E-05$ $0.303536 E-06$

$0.194833 E-00$ $0.246883 \mathrm{E}-02$ $0.177253 E-03$ $0.238087 E-04$ $0.452531 E-05$ $0.107991 E-05$ $0.303445 E-06$

$0.220810 E-00$ $0.332589 E-02$ $0.267803 E-03$ $0.393432 E-04$ $0.803377 \mathrm{E}-05$ $0.203424 E-05$ $0.601042 E-06$

$0.266723 E-00$ $0.514951 E-02$ $0.495263 E-03$ $0.832369 E-04$ $0.189545 E-04$ $0.525785 E-05$ $0.167964 \mathrm{E}-05$

$0.345717 \mathrm{E}-00$ $0.934763 E-02$ $0.113892 \mathrm{E}-02$ $0.230518 E-03$ $0.612196 E-04$ $0.193612 E-04$ $0.693291 E-05$

$0.521417 E 00$ $0.236354 \mathrm{E}-01$ $0.415678 E-02$ $0.112997 \mathrm{E}-02$ $0.385353 E-03$ $0.151951 E-03$ 
Toble 2. Spatiol Moments, Plone Isatrapic Source - Continued

n $\quad p=-1 / 2$

$1 / 2$

$3 / 2$

$5 / 2$

$7 / 2$

$9 / 2$

$11 / 2$
Palystyrene

$\ell=0 \quad$
$0.556961 \mathrm{E}-04$
$0.397290 E-00$ $0.180943 E-02$ $0.454981 \mathrm{E}$ $0.141358 \mathrm{E}-03$ $0.520289 \mathrm{E}-04$ $0.215769 \mathrm{E}-04$

Polystyrene $0.198551 E$ OI $0.626532 \mathrm{E}-01$ $0.241448 E-01$ $0.109394 E-01$ $0.549814 E-02$ $0.297399 E-02$

$0.412248 E-00$ $0.141550 \mathrm{E}-01$ $0.208407 E-02$ $0.500116 E-03$ $0.155796 \mathrm{E}-0$ 0.155 $796=03$ $0.573485 E-04$
$0.237468 E=04$

Polystyrene

$0.669579 \mathrm{E} 00$ $0.376414 E-01$ $0.748866 \mathrm{E}-02$ $0.224538 E-02$ $0.636447 \mathrm{E}-03$ $0.357784 \mathrm{E}-03$ $0.168677 E-03$

$0.403755 E-00$ $0.137137 \mathrm{E}-01$ $0.201586 E-02$ $0.484100 E=03$ $0.231057 \mathrm{E}-04$ $0.131708 E-01$
Polystyrene

$0.196823 \mathrm{E} \mathrm{OI}$ $0.219506 E-00$ $0.625948 E-01$ $0.241450 E-01$ $0.109386 E=01$ $0.549382 E-02$ $0.296822 E-02$

$0.408310 E=00$ $0.140673 \mathrm{E}=01$ $0.208344 \mathrm{E}-0$ $0.502355 E-03$ $0.157057 \mathrm{E}-03$ $0.579669 E-04$ $0.240493 E-04$ $0.151036 \mathrm{E}-03$

$0.283208 \mathrm{E}-00$ $0.625006 E-02$ $0.694030 E-03$ $0.135100 \mathrm{E}-03$ $0.356101 \mathrm{E}-04$ $0.114034 \mathrm{E}-0$ $0.419007 \mathrm{E}-05$

0.297120 E-00 $0.690776 \mathrm{E}-02$ $0.787901 E-03$ $0.155708 \mathrm{E}-03$ $0.413994 E-04$ $0.133205 E-04$ $0.490524 E-05$

$0.289198 \mathrm{E}-00$ $0.657693 \mathrm{E}-02$ $0.743515 E-03$ $0.146295 \mathrm{E}-03$ $0.388083 E-04$ $0.124723 E-04$ $0.459056 E-05$

$0.293439 E=00$ $0.679966 E-02$ $0.776883 E-03$ $0.153821 \mathrm{E}-03$ $0.153821 E-03$
$0.409575 E-04$ $0.131914 \mathrm{E}=04$ $0.486053 E-05$

MEV $=0.40$

Polystyrene

0.193766 E OI $0.218261 E-00$ $0.627574 \mathrm{E}-01$ $0.243562 E-01$ $0.110860 E-01$ $0.558835 \mathrm{E}-02$ $0.302822 E-02$

MEV $=0.70$

\begin{abstract}
0.679408 E 00 $0.391898 E-01$ $0.792111 \mathrm{E}-02$ $0.239909 E-02$ $0.899668 E-03$ $0.386541 E-03$ $0.182768 \mathrm{E}-03$
\end{abstract} $0.147299 E-01$ $0.221757 \mathrm{E}-02$ $0.540813 \mathrm{E}-03$ $0.170507 E-03$ $0.633389 E-04$ $0.264128 E-04$

Polystyrene

$0.188873 \mathrm{E} \mathrm{O}$

$0.689809 \mathrm{E} \quad 00$ $0.407720 E-01$ $0.838907 \mathrm{E}-02$ $0.257604 \mathrm{E}-02$ $0.976854 \mathrm{E}-03$ $0.423645 E-03$ 0.626699 $0.245758 E-0$ $0.112846 E-01$ $0.573187 E-02$ $0.201931 \mathrm{E}-03$

$0.301188 \mathrm{E}-00$ $0.722000 E-02$ $0.842919 E-03$ $0.169442 \mathrm{E}-03$ $0.456270 E-04$ $0.148236 E=04$ $0.549981 \mathrm{E}-05$

$0.314279 E-00$ $0.794732 \mathrm{E}-02$ $0.960810 E=03$ $0.198154 E-03$ $0.544359 E-04$ $0.733158 \mathrm{E}-04 \quad 0.179747 \mathrm{E}-04$
$0.219992 \mathrm{E}-00$ $0.346145 \mathrm{E}-02$ $0.303631 E-03$ $0.111353 \mathrm{E}-0$ $0.313079 E-05$ $0.102638 E-05$

$0.232425 E-00$ $0.390552 \mathrm{E}-02$ $0.355577 \mathrm{E}=03$ $0.589341 E-04$ $0.135435 E=04$ $0.384498 \mathrm{E}-0$ $0.126840 E-05$

$0.225329 E-00$ $0.367127 \mathrm{E}-02$ $0.329119 E-0$ $0.540459 E-04$ $0.123449 E-04$ $0.348996 \mathrm{E}-05$ $0.114782 E-05$

$0.229123 E-00$ $0.381777 E-02$ $0.346898 \mathrm{E}-03$ $0.574507 E-04$ $0.131947 \mathrm{E}-04$ $0.374345 E-05$ $0.123394 E-05$

Polystyrene

$0.184048 \mathrm{E}$ OI $0.211703 E-00$ $0.623553 \mathrm{E}-01$ $0.623553 E=01$ $0.114492 \mathrm{E}-01$ $0.586319 \mathrm{E}-02$ $0.322234 \mathrm{E}-02$

\subsection{E 00 $0.423657 \mathrm{E}=01$ $0.887136 E-02$ $0.276277 E-02$ $0.106003 E-02$ $0.464386 \mathrm{E}-03$ $0.223328 \mathrm{E}-03$}

$0.444834 E-00$
$0.170285 E-01$
$0.270213 E-02$
$0.685465 E-03$
$0.222990 E-03$
$0.850096 E-04$
$0.362408 E-04$
MEV $=2.00$

Polystyrene

$0.168928 \mathrm{E} \mathrm{OI}$ $0.197669 \mathrm{E}-00$ $0.599089 \mathrm{E}-01$ $0.243673 E-01$ $0.115575 \mathrm{E}-01$ $0.604347 \mathrm{E}-0$ $0.336491 E-02$
0.736399 E 00 $0.477535 \mathrm{E}-01$ $0.105252 \mathrm{E}-01$ $0.341351 \mathrm{E}-02$ $0.135438 \mathrm{E}-02$ $0.610544 E-03$ $0.301042 E-03$
$0.495786 E-00$ $0.215027 \mathrm{E}-01$ $0.370237 \mathrm{E}-02$ $0.998604 E-03$ $0.341179 E-03$ $0.135471 \mathrm{E}-03$ $0.597951 \mathrm{E}-04$
$0.328097 \mathrm{E}-00$ $0.874766 E-02$ $0.109529 E-02$ $0.231899 E-03$ $0.650511 \mathrm{E}=04$ $0.218542 \mathrm{E}-04$ $0.834057 \mathrm{E}-05$

$0.378345 E=00$ $0.119607 \mathrm{E}-01$ $0.167337 E-02$ $0.384848 E-0$ $0.408845 E-04$ $0.115282 E-03$ $0.163358 E-04$

$0.236082 E-00$ $0.409610 E-02$ $0.382184 E-03$ $0.645026 E-04$ $0.150264 E-04$ $0.431067 \mathrm{E}-05$ $0.143360 E-05$

$0.247924 E-00$ $0.458742 E-02$ $0.446810 E-03$ $0.778431 \mathrm{E}-04$ $0.185905 E-04$

$0.179834 E-00$ $0.211840 E-02$ $0.150436 E-03$ $0.205874 E-04$ $0.404741 E-05$ $0.100715 E-05$ $0.296425 E-06$

$0.190926 E-00$ $0.242849 \mathrm{E}-02$ $0.180677 \mathrm{E}-03$ $0.254926 E-04$ $0.511769 E-05$ $0.129231 E-05$ $0.384336 \mathrm{E}-06$

$0.184586 \mathrm{E}-00$ $0.226056 \mathrm{E}=02$ $0.164642 \mathrm{E}-03$ $0.229107 E-04$ $0.455467 E-05$ $0.114184 \mathrm{E}-05$ $0.337704 E-06$

$0.187973 E=00$ $0.236138 \mathrm{E}-02$ $0.174775 \mathrm{E}-0$ $0.245804 E-04$ $0.492200 E-0$ $0.124008 E-0$

$0.368015 E-06$ 
Table 2. Spatial Moments, Plane Isotropic Source-Continued

n

$$
p=-1 / 2
$$

$1 / 2$

$3 / 2$

$5 / 2$

$7 / 2$

Polystyrene

$M E V=4.00$

\begin{abstract}
$0.151748 E 01$ $0.181303 E-00$ $0.181303 \mathrm{E}-00$ $0.573708 E=01$
$0.243315 E-01$ $0.119923 E-01$ $0.649528 E-02$

$0.375781 E-02$
\end{abstract} $M E V=10.00$

$0.783171 E 00$ $0.557602 \mathrm{E}-01$ $0.132424 E-01$ $0.457095 E-02$ $0.191323 E-02$ $0.903853 E-03$ $0.464687 E-03$

\begin{abstract}
$0.567284 E 00$ $0.289184 \mathrm{E}-01$ $0.557353 E-02$ $0.557353 E=02$ $0.604591 E-03$ $0.255779 E-03$ $0.119341 \mathrm{E}-03$
\end{abstract}

Polystyrene

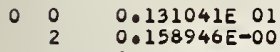
$0.535286 E-01$ $0.242518 E-01$ $0.127343 E-01$ $0.731978 \mathrm{E}-02$ $0.731978 E-02$
$0.447754 E-02$
$0.851004 E 00$ $0.694258 \mathrm{E}-01$ $0.185185 E-01$ $0.707505 E-02$ $0.324029 E-02$ $0.165969 \mathrm{E}-02$ $0.918293 E-03$
$0.682461 E 00$ $0.438542 \mathrm{E}-01$ $0.100261 E=01$ $0.340310 E-02$ $0.141318 E-02$ $0.665135 \mathrm{E}-03$ $0.665135 E-03$
$0.341420 E=03$
$0.452523 E-00$ $0.177714 \mathrm{E}-01$ $0.177714 \mathrm{E}=01$ $0.288344 \mathrm{E}=02$ $0.245128 \mathrm{E}=03$ $0.942419 E-04$ $0.403929 E-04$

$0.580966 E 00$ $0.310214 E-01$ $0.193030 E-02$ $0.739111 E-03$ 0.739111E $0.324127 \mathrm{E}-0$ $0.156194 E-03$ $0.626536 E=02$
$0.379038 E-00$ $0.119649 E-01$ $0.167210 E-02$ $0.383801 E-03$ $0.114568 \mathrm{E}-03$ $0.404271 E-04$ $0.160510 E-04$

$0.510111 E 00$ $0.233106 \mathrm{E}-01$ $0.423216 E=0$ $0.119862 \mathrm{E}-0$ $0.427491 E=0$ $0.427491 E-03$ $0.176166 \mathrm{E}-03$ $0.802865 E=04$
$0.327244 E=00$ $0.854434 E-02$ $0.104523 \mathrm{E}=02$ $0.215812 E-03$ $0.589070 E=04$ $0.192193 E-04$ $0.721324 E-05$

$0.456785 \mathrm{E}=00$ $0.182148 E=01$ $0.300793 E-02$ $0.789959 E-03$ $0.264276 E=03$ $0.102950 E=03$ $0.102950 E=03$
$0.288489 E-00$ $0.636265 E-02$ $0.689710 E-03$ $0.129231 \mathrm{E}-03$ $0.324796 E-04$

$0.985495 E-05$

$0.341694 E-05$

$0.414738 E-00$ $0.146364 E-01$ $0.221759 \mathrm{E}-02$

$0.543561 E-03$ $0.171474 E-03$ - $172474 E-03$ $0.634292 E-04$ $0.262294 E-04$ 
Toble 2. Spotial Maments, Plone Perpendicular Source

n $\quad p=-1 / 2$

$1 / 2$

$3 / 2$

$5 / 2$

$7 / 2$

$9 / 2$

$11 / 2$
- $0.200623 E_{01}$ $0.775878 E$ OD

$0.403694 E-00$

$0.229335 E-00$

$0.142350 E-00$

$0.920499 E-01$

$0.623239 E-01$

$0.432490 E-0 I$

$0.308954 E-01$

$0.224427 E-01$

$0.166376 E-01$

$0.124860 E-01$

$0.951441 E-02$
$2=6$.

$\begin{array}{ll}0.665423 E-00 & 0.398403 E-00 \\ 0.185371 E-00 & 0.866827 E-01 \\ 0.747481 E-01 & 0.287182 E-01 \\ 0.350140 E-01 & 0.114897 E-01 \\ 0.184342 E-01 & 0.527029 E-02 \\ 0.103813 E-01 & 0.263427 E-02 \\ 0.622012 E-02 & 0.141788 E-02 \\ 0.387725 E-02 & 0.803079 E-03 \\ 0.251355 E-02 & 0.476938 E-03 \\ 0.167291 E-02 & 0.293062 E-03 \\ 0.114432 E-02 & 0.186164 E-03 \\ 0.797676 E-03 & 0.121189 E-03 \\ 0.567480 E-03 & 0.8008698 E-04\end{array}$

MEV $=0.05$ $Z=6$.
. $195082 E 01$ $0.760494 \mathrm{E} 00$ $0.394309 E-00$ $0.223980 E=00$

$0.138547 \mathrm{E}=00$

$0.894289 E-01$ $0.603383 E-01$ $0.417689 E-01$ $0.297334 E-01$

$0.215393 E-01$

$0.159118 E-01$ $0.119066 \mathrm{E}-01$

$0.904111 E-02$
$0.676671 E 00$ $0.191484 \mathrm{E}-00$ $0.775606 E-01$ $0.364855 E-0$ $0.192188 \mathrm{E}-01$

$0.108325 E-01$ $0.648362 E-02$ $0.403896 E-02$ $0.261353 \mathrm{E}-02$ $0.173697 E-02$

$0.118540 E-02$ $0.824739 E-03$ $0.585221 E-03$
$0.412986 E-00$ $0.921155 E-01$ $0.309137 E-01$ $0.124927 E-01$ $0.576276 E-02$

$0.289427 E-02$ $0.156162 E-02$ $0.886444 E-03$ $0.526813 E-03$ $0.323937 \mathrm{E}-03$

$0.205702 E-03$ $0.133876 \mathrm{E}-03$ $0.892424 E-04$ $z=6$.

O.198330E OI $0.784967 \mathrm{E} 00$ $0.409145 E=00$ $0.234077 \mathrm{E}-00$ $0.145405 E-00$

$0.943347 \mathrm{E}-01$ $0.638747 E=01$ $0.443950 E-01$ $0.316988 E-01$ $0.230394 \mathrm{E}=01$

$0.170647 E-01$ $0.128054 E-01$ $0.974589 E-02$
$0.670026 E 00$ $0.190559 E-00$ $0.772044 E-01$ $0.364165 E-01$ $0.192068 \mathrm{E}-01$

$0.108528 E-01$ $0.650659 E-02$ $0.406278 E-02$ $0.263353 E-02$ $0.175403 E-02$

0.119907 E-02 $0.835883 \mathrm{E}-03$ $0.594066 E-03$

$z=6$.

$0.196614 E 01$ $0.785821 E 00$ $0.409596 \mathrm{E}-00$ $0.234950 E-00$ $0.145910 E-00$

$0.947531 E-01$ $0.641268 \mathrm{E}-0$ $0.445800 E-01$ $0.318095 E-01$ $0.231152 E-01$

$0.171068 \mathrm{E}-01$ $0.128309 E-01$ $0.975629 E-02$

MEV $=0.40$

$0.673518 E \quad 00$ $0.193501 E-00$ $0.785816 E=01$ $0.371874 E-01$ $0.196283 E-01$

$0.111066 E-01$ $0.665948 E-02$ $0.416058 E-02$ $0.269621 E-02$ $0.179589 E-02$

$0.122705 E-02$ $0.855161 E-03$ $0.607344 E-03$

$Z=6$.

0

$0.193695 E 01$ $0.787531 E$ OO $0.412566 \mathrm{E}-00$ $0.238355 E-00$ 0.148560 E-00

$0.969068 E-01$ $0.657633 E-0$ $0.458646 E-01$ $0.327963 \mathrm{E}-01$ $0.238909 E-01$

0.177115 E-01 $0.133104 E-01$

$0.679556 E 00$ $0.198716 E-00$ $0.813962 \mathrm{E}-01$ $0.388541 \mathrm{E}-01$ $0.206183 E-01$

$0.117309 E-01$ $0.706029 E-02$ $0.442820 E-02$ $0.287777 E-02$ $0.192250 E-02$

$0.131647 \mathrm{E}-02$ $0.919618 E-03$
$0.404333 E-00$ $0.698725 \mathrm{E}-01$ $0.300110 E-01$ $0.121050 E-01$ $0.557148 E-02$

$0.279656 E-02$ $0.150771 \mathrm{E}-02$ $0.855987 E-03$ $0.508690 E-03$ $0.312966 \mathrm{E}-03$

$0.198801 E-03$ $0.129479 E-03$ $0.863562 E-04$
$0.284237 E-00$ $0.507241 \mathrm{E}-01$ $0.143033 E-01$ $0.500519 E-02$ $0.203848 E-02$

$0.917319 E-03$ $0.448735 E-03$ $0.233087 E-03$ $0.127801 E-03$ $0.729669 \mathrm{E}-04$

$0.432825 E-04$ $0.264364 E-04$ $0.166152 E-04$

$0.297812 E-00$ $0.548962 \mathrm{E}-01$ $0.157929 E-01$ $0.561305 E-02$ $0.231042 E-02$

$0.104896 E-02$ $0.516276 \mathrm{E}-03$ $0.269600 E-03$ $0.148343 \mathrm{E}-03$ $0.849627 \mathrm{E}-04$

$0.504929 E-04$ $0.308939 E-04$ $0.194316 E-04$

$0.289734 E-00$ $0.528804 E-01$ $0.150702 E-01$ $0.532441 E-02$ 0.217955 E-02

$0.986006 E-03$ $0.483700 \mathrm{E}-03$ $0.252062 E-03$ $0.138419 E-03$ $0.791847 \mathrm{E}-04$

0.470035 E-04 $0.287402 \mathrm{E}-04$

$0.180644 E-04$

$0.408867 E-00$ $0.919621 E-01$ $0.308582 \mathrm{E}-01$ $0.125044 E-01$ $0.576784 \mathrm{E}-02$

0.290192 E-02 $0.156617 E-02$ $0.890291 E-03$ $0.529301 E-03$ $0.325842 E-03$

$0.206983 \mathrm{E}-03$ $0.134830 E-03$ $0.899001 E-04$

$0.416776 E-00$ $0.956897 E-0$ $0.325102 E-01$ $0.133212 E-01$ $0.619256 E-02$

$0.313823 E-02$ $0.170289 E-02$ $0.973035 E-03$ $0.580825 \mathrm{E}=0$ $0.358965 \mathrm{E}-03$

$0.228736 E-03$ $0.149461 \mathrm{E}-03$ $0.999038 E-04$

$0.293959 E-00$ $0.543781 \mathrm{E}-01$

$0.156056 E-01$ $0.554697 E-02$ $0.227881 \mathrm{E}-02$

$0.103441 E-02$ $0.508500 E-03$

$0.265533 E-03$ $0.145994 E-03$ $0.836225 E-04$

$0.496696 \mathrm{E}-04$ $0.303915 E-04$ $0.191068 E-04$

$0.220907 E-00$ $0.334119 E-01$ $0.821343 E-02$ $0.255752 E-02$ $0.937970 E-03$

$0.384246 E-03$ $0.172427 \mathrm{E}-03$

$0.827647 E-04$ $0.421687 E-04$ $0.224929 E-04$

$0.125182 E-04$ $0.720328 E-05$ $0.427937 E-05$

$0.233046 E-00$ $0.366282 \mathrm{E}-01$ $0.924108 E-02$ $0.293711 E-02$ $0.109364 E-02$

$0.453771 E-03$ $0.205608 E-03$ $0.995276 \mathrm{E}-04$ $0.510346 E-04$ $0.273776 E-04$

$0.153012 E-04$ $0.883852 E-05$ $0.526512 E-05$

$0.225809 E-00$ $0.349676 E-01$ $0.870698 E-02$ $0.274136 E-02$ $0.101213 E-02$

$0.417302 E=03$ $0.188013 E-03$ $0.906213 E-04$ $0.462873 E-04$ $0.247576 E-04$

$0.137991 E-04$ $0.795419 E-05$ $0.472895 E-05$ 
Table 2. Spatial Moments, Plone Perpendicular Source-Continued

n

$p=-1 / 2$

$1 / 2$

$3 / 2$

$5 / 2$

$7 / 2$

$9 / 2$

$11 / 2$ $z=6$. $0.415246 \mathrm{E}=00$ $0.242517 E-00$ $0.152262 E-00$

$0.100060 E-00$ $0.682933 \mathrm{E}-01$ $0.479040 E-01$ $0.344180 E-01$ $0.251921 \mathrm{E}-01$

$0.187527 E-01$ $0.141507 E-01$

$0.689981 E 00$ $0.206911 E-00$ $0.861150 E-01$ $0.416984 E-01$ $0.223617 E-01$

$0.128459 E-01$ $07 E-02$ $0.492198 E-02$ $0.321813 E-02$ $0.216238 \varepsilon-02$

$0.148820 E-02$ $0.104465 E-02$ $0.746457 \mathrm{E}-03$
$0.188794 \mathrm{E}$ OI $0.784723 E 00$

$0.430648 E-00$ $0.102020 E-00$ $0.148013 \mathrm{E}=01$

$0.358506 E-02$ $0.196619 E=02$ $0.113439 E-02$ $0.682729 E=03$ $0.425174 E-03$

$0.272736 E-03$ $0.179333 E-03$ $0.120543 E-03$ $z=6$.

$0.183977 E$ OI $0.779351 E \quad 00$ $0.417028 E-00$ $0.246111 E-00$ $0.155778 E-00$

$0.103138 E-00$ $0.708484 E-01$ $0.499951 E-01$ $0.361154 E-01$ $0.265698 E-01$

$0.198720 E-01$ $0.150629 E-01$
$0.115599 E-01$
$0.700595 E 00$ $0.215034 E-00$ $0.910255 E-01$ $0.447124 E-01$ $0.242547 E-01$

$0.140729 E-01$ $0.860829 E-02$ $0.548004 E-02$ $0.36 C 752 E-02$ $0.243929 E-02$

$0.168844 E-02$ $0.119158 E-02$
$0.445051 E-00$ $0.108634 E-00$ $0.386246 \mathrm{E}-01$ $0.164440 E-01$ $0.788124 E-02$

$0.410124 E-02$ $0.227544 E-02$ $0.132630 \mathrm{E}-02$ $0.805497 E-03$ $0.505761 E-03$

$0.326855 E-03$ $0.216399 E-03$ $0.146382 E-03$ $z=6$.

$0.168856 \mathrm{E}$ ol $0.745520 E$ OO $0.413272 E-00$ $0.250831 E-00$ $0.162281 E-00$

$0.109636 E-00$ $0.766172 E-01$ $0.549461 E=01$ $0.402654 E-01$

$0.300307 E-01$

$0.227420 E-01$

$0.174461 E-01$

$0.135382 E-01$

$$
\text { MEV }=4.00
$$

$0.151704 E 01$ $0.710917 E$ OO $0.410431 E-00$ $0.259722 \mathrm{E}-00$ $0.174128 E-00$

$0.121481 E-00$ C. $873658 E-01$ $0.643352 E-01$ C. $483073 E-0 I$ C. 368607 E-0I

$0.285174 \mathrm{E}-01$ $0.223250 E-02$ $0.176605 \mathrm{E}-01$

C.131270E OI C. $651212 \mathrm{E} \mathrm{OO}$ $0.401648 E-00$ $0.270937 E-00$ C. $192561 \mathrm{E}-00$ $0.141749 \mathrm{E}-00$ C. $107094 \mathrm{E}=00$ $0.825615 E-01$ $0.647000 E=01$ $0.513958 \overrightarrow{\mathrm{E}}-01$

$0.413020 E-01$ $0.335225 E-01$
$0.736579 E \quad 00$ $0.241768 E-00$ $0.107601 E-00$ $0.551498 E-01$ $0.309520 E-0]$

$0.184990 \mathrm{E}=01$ $0.116030 E-01$ $0.755515 \mathrm{E}-02$ $0.507326 \mathrm{E}-02$

$0.349374 E-02$

$0.245857 E-02$ $0.176216 E-02$ $0.128357 E-02$ $z=6$.

$0.783300 E$ OO $0.279926 E=00$ $0.2733749 E=00$ $0.728698 \mathrm{E}-01$ C. 43071 OE-OI

\section{$0.269368 E-01$} $0.175828 \mathrm{E}-01$ C. $118674 \mathrm{E}-01$ $0.523189 \mathrm{E}-02$ $0.584086 \mathrm{E}-02$

\section{$0.422512 E-02$} C. $310731 E-02$

$0.231860 E-02$

$z=6$.

$0.496049 E-00$ $0.132702 E-00$ $0.506534 E-01$ $0.228826 \mathrm{E}=01$ $0.115128 E-01$

$0.624697 E-02$ $0.216089 E-02$ $0.134940 \mathrm{E}-02$ $0.368966 E-03$

$0.574496 E-03$ $0.388430 E-03$ $0.267849 E-03$

$0.567490 E \quad 00$ $0.170542 E-00$ $0.716391 E-01$ $0.351120 E-01$ $0.189379 \mathrm{E}-01$

$0.109191 \mathrm{E}-01$ $0.062226 \mathrm{E}-02$ C. $417909 \mathrm{E} \rightarrow 02$ $0.272446 E-02$ $0.182481 E=02$

$0.125073 E-02$ $0.074410 E-03$ C.622020E-03 $0.240074 E-00$ $0.115752 \mathrm{E}-00$ $0.640418 \mathrm{E}-0$ $0.384454 E-01$

$0.243967 \mathrm{E}-01$ C. $161320 E=01$ $0.110147 \mathrm{E}-01$ $0.771869 E-02$ $0.552705 E-02$

$0.234445 E$ O. $172120 E-01$ C.128731E-0I

$0.778104 E-02$ $0.753319 E-02$ $0.587122 E-02$

$0.403102 E-02$ $0.298680 \mathrm{E}-02$ $0.354420 E=01$ $0.698296 E-02$ $0.359178 \mathrm{E}-02$

$0.680953 E 00$

$0.314500 E-00$ $0.617800 E-01$ $0.185161 \mathrm{E}-01$ $0.682984 E-02$ $0.288844 E-02$ $0.134511 E-02$
$0.675240 E-03$ $0.359360 E=03$ $0.200772 E-03$ $0.116713 E=03$

$0.702153 E=04$ $0.434808 E-04$ $0.276242 E=04$

$0.328306 E-00$ $0.668357 E-01$ $0.206105 E-01$ $0.778555 E=02$ $0.335869 \mathrm{E}-02$

$0.159102 E-02$ $0.810530 E-03$ $0.437000 E=03$ $0.246979 \mathrm{E}-03$ $0.145074 \mathrm{E}-03$

$0.881021 E-04$ $0.550297 \mathrm{E}-0$ $0.352399 \mathrm{E}-04$

$0.378611 E=00$ $0.862225 E=01$ $0.290584 E-0$ $0.118254 E-01$ $0.542854 \mathrm{E}-02$

$0.271340 E-02$ $0.144779 E-02$ $0.813281 \mathrm{E}-03$ $0.476614 E-0$ $0.289301 E-03$

$0.180963 E-03$ $0.116148 E-03$ $0.762512 \mathrm{E}-04$

$0.45274,3 E-00$ $0.118835 E=00$ $0.450107 \mathrm{E}=01$ $0.450107 E=01$ $0.202348 E-01$
$0.101192 E-01$

$0.545210 E-02$ C. $310843 E-02$ $0.185301 E=02$ $0.114572 \mathrm{E}-02$ $0.730310 \mathrm{E}-03$

$0.477781 E=03$ $0.319653 E-03$ $0.218101 \mathrm{E}-03$

$0.579212 \mathrm{E} 00$ $0.184960 E-00$ $0.826969 E-01$ $0.429708 \mathrm{E}-01$ $0.244235 E-01$

$0.147578 E-01$ C. $933191 E-02$ $0.611394 E-02$ $0.412260 E-02$ $0.284718 E-02$
$0.200680 E-02$ $0.143953 E-02$

$0.248124 E-00$ $0.416952 E=01$ $0.110177 \mathrm{E}=01$ $0.364885 E-02$ $0.140191 \mathrm{E}-02$ $0.598676 E-03$
$0.277611 E=03$ $0.137339 E=03$ $0.717020 E-04$ 
Toble 2. Spotial Moments, Plone Perpendiculor Source-Continued

n $\quad p=-1 / 2$

$1 / 2$

$3 / 2$

$5 / 2$

$7 / 2$

$9 / 2$

$11 / 2$
$2=13$.

$\ell=0$

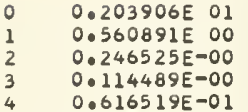

$0.658958 \mathrm{E} 00$ $0.141344 \mathrm{E}-00$ $0.482409 E-01$ $0.188148 \mathrm{E}-01$ $0.861956 E-02$

$0.341734 E-01$ $0.204383 \mathrm{E}-01$ $0.124584 \mathrm{E}-01$ $0.796245 E-02$ $0.515980 E-02$ $0.223083 E-02$ $0.122910 \mathrm{E}-02$ $0.715314 \mathrm{E}-03$

$0.345534 E-02$ $0.233870 \mathrm{E}-02$

$0.264418 E-03$ $0.166829 \mathrm{E}-03$ $0.108302 E-03$
$0.419965 E-02$ $0.426662 E-03$ $Z=13$. $0.235139 \mathrm{E}=00$ $0.108350 E=00$ $0.575659 \mathrm{E}-01$

$0.316229 E-01$ $0.186833 E-01$ $0.112824 \mathrm{E}-01$ $0.712875 \mathrm{E}-02$ $0.457603 E-02$

$0.303116 E-02$ $0.203234 E-02$ $0.139488 E-02$ MEV $=0.10$ $0.491161 \mathrm{E}=01$ $0.221114 \mathrm{E}-02$

\section{$0.198161 \mathrm{E} 01$ $0.550135 \mathrm{E} \mathrm{00}$ $0.237612 \mathrm{E}-00$ $0.110031 E=00$ $0.584098 \mathrm{E}-01$ \\ $0.321740 E-01$ $0.189949 \mathrm{E}-01$ $0.114879 \mathrm{E}-01$ $0.725261 E-02$ $0.465897 \mathrm{E}-02$ \\ $0.308322 \mathrm{E}-02$ $0.206768 \mathrm{E}-02$} $0.141764 \mathrm{E}-02$

$0.670368 E 00$ MEV $=0.20$

0.196899 E 01 $0.555017 E \quad 00$ $0.239490 E-00$ $0.111623 E-00$ $0.592385 \mathrm{E}-01$

$0.327506 E-01$ $0.193308 E-01$ $0.117170 \mathrm{E}=01$ $0.739470 \mathrm{E}-0$ $0.475657 \mathrm{E}-02$

$0.314628 \mathrm{E}-02$ $0.211153 E-02$ $0.144681 E-02$ $M E V=0.40$ $0.430890 E=03$

$0.193635 E \quad 01$ $0.560952 E$ OO $0.242860 \mathrm{E}-00$ $0.114620 E-00$ $0.610421 \mathrm{E}-01$

$0.340165 E-01$ $0.201408 E-01$ $0.122778 E-01$ $0.776954 \mathrm{E}-02$ $0.501960 E-02$

$0.332794 \mathrm{E}-02$ $0.224127 \mathrm{E}-02$ $0.153875 \mathrm{E}-02$
$0.670578 E \quad 00$ $0.144828 E=00$ $0.190939 E-01$ $0.867334 \mathrm{E}-02$

$0.420053 E-02$ $0.120945 \mathrm{E}-02$ $0.697376 E-03$ $0.412695 \mathrm{E}-03$

$0.253392 E-03$ $0.158566 E-03$ $0.101989 E-03$ $z=13$. $0.146071 E-00$ $0.494515 E-01$ $0.192869 E-01$ $0.875133 \mathrm{E}=02$

\section{$0.424612 E-02$} $0.223310 E=02$ $0.122268 E-02$ $0.704343 E-03$ $0.416987 \mathrm{E}-03$

$0.255764 E-03$ $0.160047 \mathrm{E}-03$ $0.102825 E-03$ $Z=13$.

$0.672936 E 00$ $0.148675 E-00$ $0.503902 E=01$ $0.197707 \mathrm{E}-01$ $0.897652 \mathrm{E}-02$

$0.437081 \mathrm{E}-02$ $0.229936 E-02$ $0.126173 E-02$ $0.726838 E=03$

$0.264225 E-03$ $0.165470 E=03$ $0.106261 E-03$ $z=23$.

$0.405047 E-00$ $0.72212 \mathrm{E}-01$ $0.204189 E-01$ $0.690198 E-02$

$0.119570 \mathrm{E}-02$ $0.569007 E=03$ $0.284873 E-03$ $0.151273 E-03$ $0.831181 \mathrm{E}-04$

$0.476028 E-04$ $0.279456 E-04$ $0.169223 \mathrm{E}-04$

$0.404776 E-00$ $0.726769 E-01$ $0.205123 E-01$ $0.694764 E-02$ $0.276787 \mathrm{E}-02$

$0.120324 E-02$ $0.571952 \mathrm{E}-03$ $0.286502 \mathrm{E}-03$ $0.151973 E-03$ $0.835109 E-04$

$0.477732 E-04$ $0.280388 E-04$ $0.169581 E=04$

$0.408108 E-00$ $0.742974 E-01$ $0.210347 \mathrm{E}-01$ $0.716893 E-0$

$0.124848 E-02$ $0.594068 E-03$ $0.298325 \mathrm{E}-03$ $0.158328 \mathrm{E}-03$ $0.871440 E-04$

$0.498591 E=04$ $0.292923 E-04$

$0.416941 E-00$ $0.780799 E-01$ $0.224113 E-01$ $0.775758 E-0$ $0.312403 E-02$ $0.248577 \mathrm{E}-02$ $0.137266 E-02$ $0.793614 E-03$ $0.472766 E-03$

$0.290764 E-03$ $0.182797 E-03$ $0.117678 E-03$

$0.137756 E-02$ $0.659831 E=03$ $0.333884 E-03$ $0.178104 E-03$ $0.986062 \mathrm{E}-04$

$0.566465 E-04$ $0.334365 E-04$ $0.202871 \mathrm{E}-04$ $0.177138 E-04$
$0.390161 E-00$ $0.683960 E-01$ $0.192809 \mathrm{E}=01$ $0.649958 \mathrm{E}-02$ $0.259981 E-02$

$0.113096 E-02$ $0.540876 E-03$ $0.271904 \mathrm{E}-03$ $0.145291 E-03$ $0.802685 E-04$

$0.462930 E-04$ $0.273483 E-04$ $0.16684 \mathrm{IE}=04$ $0.275356 E-02$ $0.286094 \mathrm{E}-02$

$0.276646 \mathrm{E}-00$ $0.409665 E=01$ $0.989262 \mathrm{E}-02$ $0.294300 \mathrm{E}-02$ $0.104740 E-02$

$0.411974 E-03$ $0.179350 \mathrm{E}-03$ $0.829333 E=04$ $0.409782 \mathrm{E}-04$ $0.210862 E-04$

$0.113733 E-04$ $0.631683 E=05$ $0.363468 E-05$

$0.290399 E-00$ $0.440978 \mathrm{E}-01$ $0.107657 E=01$ $0.322972 E-02$ $0.115278 E-02$

$0.454567 E-03$ $0.197737 E-03$ $0.913700 \mathrm{E}-04$ $0.913700 E=04$
$0.450122 E-04$ $0.230983 E-04$

$0.124039 E-04$ $0.686113 E-05$ $0.392693 E-05$

$0.290147 E-00$ $0.443178 E=01$ $0.107985 E-01$ 0.32432 OE-02 $0.115563 \mathrm{E}-02$

$0.455908 \mathrm{E}-03$ $0.198054 \mathrm{E}-03$ $0.915287 \mathrm{E}-0.4$ $0.450344 E-04$ 0.231056 E-04

$0.123922 E-04$ $0.685263 \mathrm{E}-05$ $0.391635 \mathrm{E}-05$

$0.293251 E-00$ $0.454302 E-01$ $0.111216 \mathrm{E}-01$ $0.336249 E-02$ 0.120140 E-O2

$0.475995 E-03$ $0.207149 E-03$ $0.960106 E-04$ $0.472922 E-04$ 0.24312 OE-04

$0.130472 E-04$ $0.722320 \mathrm{E}-05$ $0.412972 E-05$

$0.301525 \mathrm{E}-00$ $0.481457 E-01$ $0.119960 \mathrm{E}=01$ $0.369189 \mathrm{E}-02$ $0.133484 \mathrm{E}-02$

$0.214169 E=00$ $0.274409 E-01$ $0.581444 E-02$ $0.155048 E-02$ $0.497977 E-03$

$0.178912 \mathrm{E}-03$ $0.715414 E-04$ $0.306396 E-04$ $0.140849 E-04$ $0.678366 \mathrm{E}-05$

$0.343691 E-05$ $0.180121 E-05$ 0.980757 E-06

$0.226403 E-00$ $0.299475 E-01$ $0.645705 E-02$ $0.174515 E-02$ 
Toble 2. Spotiol Moments, Plone Perpendicular Source - Continued

n

$p=-1 / 2$

$1 / 2$

$3 / 2$

$5 / 2$

$7 / 2$

$9 / 2$

$11 / 2$
$2=13$.

$0.187981 E 01$ $0.566164 \mathrm{E} \mathrm{OO}$ $0.247399 \mathrm{E}=00$ $0.118898 \mathrm{E}=00$ $0.638515 \mathrm{E}-01$

$0.360121 E-01$ $0.214784 E-01$

$0.132138 \mathrm{E}-01$

$0.841563 E-02$

$0.547805 \mathrm{E}=02$

$0.365262 E-02$

$0.247578 \bar{E}-02$

$0.170847 E-02$

MEV $=1.00$

$\begin{array}{ll}0.691746 E-00 & 0.433023 E-00 \\ 0.163275 E-00 & 0.847478 E-01 \\ 0.568825 E-01 & 0.249941 E-01 \\ 0.231038 E-01 & 0.888019 E-02 \\ 0.106904 E-01 & 0.364211 E-02 \\ & \\ 0.532622 E-02 & 0.163500 E-02 \\ 0.284287 E-02 & 0.793844 E-03 \\ 0.158684 E-02 & 0.407149 E-03 \\ 0.924942 E-03 & 0.219538 E-03 \\ 0.555814 E-03 & 0.122864 E-03 \\ 0.344218 E-03 & 0.712154 E-04 \\ 0.217995 E-03 & 0.424153 E-04 \\ 0.141188 E-03 & 0.259320 E-04\end{array}$

$2=13$.

$0.182231 E$ OI

$0.568399 E$ OO

$0.251021 E-00$

$0.122626 E=00$

$0.664483 E-01$

$0.378910 E-01$

$0.227731 E-01$

$0.141308 E-01$

$0.906012 E-02$

$0.593989 E-02$

$0.398422 E-02$

$0.271736 E-02$

$0.188528 E-02$

MEV $=2.00$

$0.704539 \mathrm{E} 00$

$0.172613 E-00$

$0.613611 \mathrm{E}=01$

$0.254381 E-01$

$0.119342 \mathrm{E}-01$

$0.602870 E-02$

$0.325195 \mathrm{E}-02$

$0.183443 E-02$

$0.107853 E-02$

$0.653715 E-03$

$0.407839 E-03$

$.260189 E-03$

$0.169606 E-03$ $2=13$.

$0.167676 E$ OI $0.573559 E$ OO $0.263196 E=00$

$0.134599 E-00$

$0.752097 \mathrm{E}-01$

$0.442924 E-01$

$0.27313 \mathrm{CE}-01$

$0.173932 E-0]$

$0.114034 E-01$

$0.764399 E-02$

$0.523013 E-02$

$0.36378 \mathrm{CE}=02$

$0.256976 E-02$

$0.739576 E$ OO

$0.200308 E-00$

$0.757799 E-01$

$0.333040 E-01$

C. 103208 E-01

$0.858878 E-02$

$0.479173 E-02$

$0.279099 E-02$

$0.168730 \mathrm{E}-02$

$0.105039 E-02$

$0.671234 E-03$

$0.438254 E-03$

$0.291808 E-03$ $M E V=4.00$

$z=13$.

$0.150159 E 01$ $0.572468 E$ OO $0.279520 E-00$ $0.151933 \mathrm{E}-00$ $0.890286 E=01$

$0.548428 E-01$ C. $351621 E-01$ $0.232361 E-01$

$0.157540 E-01$

$0.109048 E-01$

$0.767877 E \quad 00$ $0.242184 \mathrm{E}-00$ $0.100374 \mathrm{E}-00$ $0.478173 E-01$ $0.250165 E-01$

$0.139612 E-01$ $0.019614 E-02$ $0.500322 E-02$ $0.315537 E-02$ $0.204368 E-02$

$0.135458 E-02$ . $768693 E-02$ $0.915562 E-03$ $0.399315 E-02$

$0.629695 E-03$ $M E V=10 \cdot C 0$ $z=13$.

$0.129644 E$ OI $0.562491 E 00$ $0.304850 E-00$ $0.182307 E-00$ $0.115994 E-00$

$0.770350 E-02$ $0.528664 E-01$ $0.372220 E-01$ $0.267668 \mathrm{E}-01$ C. $195835 E-01$

$0.145528 E-01$ $0.109526 E-01$ $0.833801 E-02$
$0.356174 E \quad 00$ $0.311597 E-00$ $0.140861 E=00$ $0.803939 E-01$ $0.468728 E-01$

$0.288250 E-01$ $0.124611 E-01$ $0.122067 E-01$ $0.328565 E-02$ $0.574002 E-02$

$0.40>276 E-02$ $C . \angle 9161 C E-U 2$ $0.21260 C E-02$
$0.691847 E 0 O$
$0.450476 \mathrm{E}-00$ $0.920220 E=01$ $0.279288 \mathrm{E}-01$ $0.101839 E-01$

$0.425868 E-02$

$0.194641 E-02$ $0.958771 \mathrm{E}-03$ $0.498477 \mathrm{E}-03$ $0.271885 E-03$

$0.153840 E-03$

$0.900253 E=04$ $0.541147 E=04$ $0.333569 E=04$

$0.500452 E \quad 00$ $0.114760 E-00$ $0.378565 E-01$ $0.148548 E-01$ $0.658325 E-02$

$0.317009 E-02$ $0.163183 E-02$ $0.883537 \mathrm{E}-03$ $0.499391 \mathrm{E}-03$

$0.292161 E-03$

$0.176193 E-03$ $0.108974 E-03$ $0.689538 E-04$

$0.574821 E 00$ $0.153087 E-00$ $0.567412 E-01$ $0.246255 E-01$ $0.118675 E-01$

$0.615493 E-02$ $0.338047 E-02$ $0.194146 E-02$ $0.115725 E-02$ $0.711219 E-03$

$0.448805 E-03$ $0.289656 E-03$

$0.190709 E=03$

$0.316764 E-00$ $0.530956 E-0$ $0.136837 \mathrm{E}-01$ $0.434277 \mathrm{E}-02$ $0.160714 \mathrm{E}-02$

$0.658760 E-03$ $0.294236 E-03$ $0.139913 E-03$ $0.703323 E-04$ $0.368973 E-04$

$0.201312 E-04$ $0.113319 \mathrm{E}-04$ $0.656889 E-05$

$0.333553 E=00$ $0.586621 E-01$ $0.156635 E-01$ $0.512814 E-02$ $0.194497 \mathrm{E}-02$

$0.815038 E-03$ $0.370771 E-03$ $0.179308 E-03$ $0.914531 E-04$ $0.486342 E-04$

$0.268546 E-04$ $0.152892 E-04$ $0.895362 E-05$

$0.383056 E-00$ $0.767045 E-01$ $0.226402 \mathrm{E}-01$ $0.808553 \mathrm{E}-02$ $0.329292 E-02$

$0.146995 E-02$ $0.705938 E-03$ $0.358662 E-03$ $0.191098 E-03$

$0.105824 E-03$

$0.606147 E-04$ $0.006147 E-04$ $0.215891 E-04$

$04460590 E-00$ $0.109311 E-00$ $0.370087 E-01$ $0.148771 E=01$ $0.669737 \mathrm{E}-02$

$0.326654 E-02$ $0.169584 E-02$ $0.924638 E-03$ $0.525118 E=03$ $0.308446 E-03$

$0.186523 E-03$ $0.115633 E-03$ $0.732801 E-04$ $0.226015 E-00$ $0.996151 E-01$ $0.503201 E-01$ $0.276734 E-01$

$0.161496 E-01$ $0.986044 E-02$ $0.623876 \Xi-02$ $0.406458 \mathrm{E}-02$ $0.271350 E=02$

$0.184981 E-02$ $0.128349 E-02$ $0.905541 E-03$

$0.250183 E-00$ $0.367175 E-01$

$0.843863 E-02$ $0.242742 E-02$ $0.820864 E-03$

$0.310268 E=03$ $0.128568 \mathrm{E}=03$ $0.570772 E=04$ $0.269114 \mathrm{E}-04$ $0.133030 E-04$

$0.686350 E-05$ $0.366616 E-05$ $0.202232 E-05$

$0.265546 E-00$ $0.410761 \mathrm{E}-01$ $0.983424 E-02$ $0.293151 E-02$ $0.102063 E-02$

$0.395928 E-03$ $0.167718 E-03$ $0.759680 \mathrm{E}=04$ $0.364512 E-04$ $0.183139 E-04$

$0.958640 E-05$ $0.519061 E-05$ $0.289859 E-05$

$0.311804 E-00$ $0.555952 E-01$ $0.149184 \mathrm{E}-01$ $0.490912 E-02$ $0.185678 E-02$

$0.775147 \mathrm{E}-03$ $0.349963 E-03$ $0.167945 E-03$ $0.848442 E-04$ $0.447031 E-04$

$0.244329 E-04$ $0.137744 E-04$ $0.798358 E-05$

$0.387016 E=00$ $0.832301 E-01$ $0.260649 E-01$ $0.980345 E-02$ $0.415842 E-02$

$0.192162 E-02$ $0.949215 E-03$ 
Table 2. Spotial Moments, Plane Perpendicular Source-Continued

n

$p=-1 / 2$

$1 / 2$

$3 / 2$

$5 / 2$

$7 / 2$

$9 / 2$

$11 / 2$

MEV $=0.025 \quad Z=290$

\begin{tabular}{|c|c|c|}
\hline$\ell=0$ & 0 & $0.216947 \mathrm{E} 01$ \\
\hline & 1 & $0.353260 E=00$ \\
\hline & 2 & $0.134714 E-00$ \\
\hline & 3 & $0.452376 E-01$ \\
\hline & 4 & $0.210711 E-01$ \\
\hline & 5 & $0.914676 E-02$ \\
\hline & 6 & $0.475547 \mathrm{E}-02$ \\
\hline & 7 & $0.237059 \mathrm{E}-02$ \\
\hline & 8 & $0.132650 E-02$ \\
\hline & 9 & $0.722547 E-03$ \\
\hline & & $0.426740 E=03$ \\
\hline & & $0.247482 E-03$ \\
\hline & 2 & $0.152419 E-03$ \\
\hline
\end{tabular}

$0.634619 E \quad 00$
$0.873889 E-01$
$0.249486 E-01$
$0.725027 E-02$
$0.283837 E-02$
$0.110081 E-02$
$0.506332 E-03$
$0.230472 E-03$
$0.117323 E=03$
$0.592318 E-04$
$0.323820 E-04$
$0.175945 E-04$
$0.101499 E-04$

$0.360038 E-00$ $0.425694 \mathrm{E}-01$ $0.978854 E-02$ $0.249685 E-02$ $0.842541 E-03$ $0.121046 E-03$ $0.505317 E-04$ $0.110487 E-04$

$0.561193 E-05$ $0.155053 E-05$
$0.294128 E-03$ $0.235438 E-04$ $0.286252 E-05$

$0.249416 E-00$ $0.257620 \mathrm{E}-01$ $0.499731 E-02$

$0.113522 \mathrm{E}-02$

$0.33718 a E-03$

$0.106858 E-03$ $0.397805 E-04$ $0.153127 E-04$ $0.657178 \mathrm{E}-05$ $0.287711 \mathrm{E}-05$

$0.136334 E-05$ $0.654549 E-06$ $0.333856 \mathrm{E}-06$

MEV $=0.05$ $z=29$.

$\begin{array}{ll}0 & 0.203129 E \quad 01 \\ 1 & 0.314083 E-00 \\ 2 & 0.113059 E-00 \\ 3 & 0.361477 E-01 \\ 4 & 0.159719 E-01\end{array}$

$0.159719 E-01$

$0.661714 E-02$ $0.327440 E-02$ $0.156060 E-02$ $0.833313 E-03$

$0.434610 E-03$

$0.245461 E-03$ $0.136475 E-03$ $0.805204 E-04$

$0.660475 E 00$ $0.660475 \mathrm{E} 00$ $0.881791 \mathrm{E}-01$
$0.243071 \mathrm{E}-01$ $0.600550 E-02$ $0.255940 E-02$ $0.459883 E-01$ $0.104363 \mathrm{E}-01$ $0.260233 E-02$ $0.856186 E-03$

$0.954830 E-03$ $0.421401 \mathrm{E}-03$ $0.184408 E-03$ $0.900864 \mathrm{E}-04$ $0.437273 E-04$

$0.229565 E-04$ $0.119966 E-04$ $0.665139 E-05$

$0.290564 E-03$ $0.115949 E-03$ $0.469011 E-04$ $0.211330 E-04$ $0.959228 E-05$

$0.470638 E-05$ $0.231988 E-05$ $0.121335 E-05$ MEV $=0.10$ $z=29$.

$0.201498 E$ OI $0.306929 E-00$ $0.108722 E-00$ $0.343618 E-01$ $0.149447 E-01$

$0.612762 E-02$ $0.293559 E-02$ $0.140886 E-02$ $0.741066 E-03$ $0.382742 E-03$

$0.663686 E_{0} 00$ $0.876050 E-01$ $0.237994 \mathrm{E}-01$ $0.659808 E-02$ $0.244577 \mathrm{E}-02$

$0.904007 E-03$ $0.393261 E-03$ $0.170514 \mathrm{E}-03$ $0.821247 \mathrm{E}-04$ $0.394957 \mathrm{E}-04$

$0.213044 E-03$ $0.117311 E-03$

$0.204491 E-04$ 0.105876 E-04 $0.579123 E-05$

$0.396179 E-00$ $0.461053 E-01$ $0.103308 E-01$ $0.255529 E-02$ $0.830007 E-03$

$0.279441 E-03$ $0.110064 E-03$ $0.441543 E-04$ $0.196362 E-04$ $0.883720 E-05$

$0.427988 E-05$ $0.209135 E-05$ $0.107990 E-05$ $M E V=0.20$ $z=29$.

0 $0.198355 E$
$0.304265 E-00$ $0.106473 E=00$ $0.337182 E-01$ $0.145119 E-01$

$0.594742 E-02$ $0.287024 E-02$ $0.135170 E-02$ $0.704666 \mathrm{E}-03$ $0.362833 E-03$

$10 \quad 0.200252 E-03$ $\begin{array}{ll}11 & 0.109852 E-03 \\ 12 & 0.633861 E-04\end{array}$

MEV $=0.40$

0 $0.308599 \mathrm{E}-00$ $0.308599 \mathrm{E}=00$
$0.107369 \mathrm{E}-00$ $0.346926 E-0$ $0.148883 E-01$

$0.618472 \mathrm{E}-02$ $0.298005 E-02$ $0.141706 E-02$ $0.738027 E-03$ $0.382755 E-03$

$10 \quad 0.211104 E-03$

$11 \quad 0.116443 E-03$

0.671510 E-04
$0.669975 E 00$ $0.892455 E-01$ $0.240893 E-01$ $0.669993 E-02$ 0.246560 E-02

$0.911746 E-03$ $0.393665 E-03$ $0.170461 E=03$ $0.814840 E-04$ $0.390880 \mathrm{E}-04$

$0.200880 E-04$ $0.103659 E-04$ $0.562834 E-05$ $z=29$.

$0.404267 E-00$ $0.476253 E-01$ $0.106583 E-01$ $0.265032 E-02$ $0.857732 E-03$

$0.289385 E-03$ $0.113417 E-03$ $0.454976 E-04$ $0.201195 E-04$ $0.904120 E-05$

$0.435234 E-05$ $0.212144 E-05$ $0.108862 E-05$

$0.678134 E \quad 00$ $0.934421 E-01$ $0.252966 \mathrm{E}-01$ $0.717876 \mathrm{E}-02$

$0.264588 \mathrm{E}-02$

$0.991991 E-03$ $0.428761 E-03$ $0.187522 \mathrm{E}-03$ $0.897074 E-04$ $0.433581 E-04$

$0.222939 E-04$ $0.115709 E-04$
$0.424906 E-00$ $0.506347 E-01$ $0.290973 E-02$ $0.947585 E-03$

$0.324608 \mathrm{E}-03$ $0.127759 E-03$ $0.518281 E-04$ $0.229881 E-04$ $0.104187 E-04$

$0.502663 E-05$ $0.246640 E-05$ $0.126773 E-05$ $0.114497 E-01$ $0.628471 E-05$
$0.278414 E-00$ $0.291046 E-01$ $0.566974 E-02$ $0.127636 \mathrm{E}-02$ $0.374699 E-03$

$0.116695 E-03$ $0.425680 E-04$ $0.160115 E-04$ $0.670055 \mathrm{E}-05$

$0.285643 \mathrm{E}-05$

$0.131605 E-05$ $0.613908 \mathrm{E}-06$ $0.303953 E-06$

$0.282183 E-00$ $0.293570 \mathrm{E}-01$ $0.565612 \mathrm{E}-02$ $0.126500 \mathrm{E}-02$ $0.367197 \mathrm{E}-03$

$0.113591 E-03$ $0.409537 E-04$ $0.152928 \mathrm{E}-04$ $0.267495 E-05$

$0.121764 E-05$ $0.563442 E-06$ $0.275631 E-06$

$0.289673 E-00$ $0.305964 E-01$ $0.591156 E-02$

$0.133195 \mathrm{E}-02$

$0.386452 E-03$

$0.120015 E-03$ $0.431630 E-04$ $0.161409 E-04$ $0.664945 E-05$ $0.281203 E-05$

$0.127430 E-05$ $0.286592 E-06$

$0.299613 E-00$ $0.3284025-01$ $0.644699 E-02$ $0.148737 E-02$ $0.436048 E-03$

$0.137738 E-03$ $0.498989 E-04$ $0.188985 E-04$ $0.782717 E-05$ $0.334267 E-05$

$0.152091 E-05$ $0.708166 E-06$ $0.345779 E=06$ $0.632353 E-05$ $0.588781 E-06$

$0.190304 E-00$ $0.174285 E-01$ $0.293967 E-02$ $0.602184 E-03$ $0.160047 E-03$

$0.464263 E-04$ $0.157797 E-04$ $0.563054 \mathrm{E}-05$ $0.223917 E-05$ $0.917935 E-06$

$0.407429 E-06$ $0.184610 E-06$ $0.889226 E-07$

$0.215735 E-00$ $0.203362 E-01$ $0.349307 E-02$ $0.717715 \mathrm{E}-03$

$0.549335 E-04$ $0.184802 E-04$ $0.649785 E-05$ $0.254030 E-05$ $0.102104 E-05$

$0.443587 E-06$ $0.196412 E-06$ $0.923446 E-07$

$0.219080 E-00$ $0.206050 E=01$ $0.350553 E-02$ $0.716516 E-03$ $0.188582 E-03$

$0.539961 E-04$ $0.179765 E-04$ 
Toble 2. Spatial Moments, Plane Perpendicular Source-Continued

$0.674346 E-02$
$0.188548 E$ OI $0.317061 \varepsilon-00$ $0.110227 \mathrm{E}-00$

$0.368331 \varepsilon-01$

$0.158588 \varepsilon-01$ $0.326536 E-02$ $0.158042 E-02$ $0.827667 E=03$

$0.435365 E-03$

$0.241478 E-03$

$0.134777 E-03$

$0.701555 E-04$

MEV $=1.00$
$0.690514 E 00$ $0.275947 E-01$ $0.810123 E-02$ $0.301558 E-02$

$0.115807 E-02$ $0.504955 E-03$ $0.224946 E-03$ $0.108476 E-03$ $0.532130 E-04$

$0.275638 E-04$ $0.144846 E-04$ $0.792114 E-05$ $0.100632 E=00$

$0.431365 \varepsilon=00$ $0.557714 E-01$ $0.129181 \varepsilon-01$ $0.340545 E-02$ $0.112783 E-02$

$0.396687 E-03$ $0.158226 E-03$ $0.655109 E-04$ $0.293865 E-04$ $0.135403 E-04$

$0.659720 E-05$ $0.328205 E-05$ 0.170177 E-05

$z=29$.

0

$0.182812 E \quad 01$ $0.324857 E-00$ 0.113198 E-00 $0.390279 E-01$ $0.169016 E=01$

$0.734395 E-02$ $0.358124 E-02$ $0.176214 E-02$ $0.929619 E-03$ $0.495588 E-03$

$0.276873 E-03$ $0.156294 \mathrm{E}-03$

$0.703219 E \quad 00$ $0.108204 E-00$ $0.914074 E-02$ $0.344476 E-02$

$0.135347 E-02$ $0.596591 \varepsilon-03$ $0.270496 E-03$ $0.131730 E-03$ $0.655568 \mathrm{E}-04$

$0.342647 E-04$ $0.182268 E-04$ $0.912673 E-04$

$0.100507 E-04$ $M E V=2.00$ $z=29$.

- 0 0.167823E OI $0.347708 E=00$ $0.123808 E-00$ $0.464463 E-01$ a. $206823 \bar{E}-01$

$0.953182 E-02$ $0.478427 E-02$ $0.246480 E-02$ C. $133729 \mathrm{E}-02$ $\checkmark .740716 E=03$

$0.425002 E-03$ $0.247943 E-03$

$0.148512 \mathrm{E}-03$
$0.448657 E-00$ $0.613753 E=01$ $0.146006 E-01$ $0.398879 E-02$ $0.134644 E-02$

$0.485892 E-03$ $0.196802 E-03$ $0.831262 E-04$ $0.377784 E-04$ 0.176936 E-04

$0.872014 E-05$ $0.439863 E-05$ $0.230420 E-05$
0.499899E-00 $0.801521 E-01$ $0.207407 E-01$ $0.626346 E-02$ $0.224971 E-02$ $0.132451 E=00$ $0.129550 E-01$ $0.510663 E-02$

$0.213928 E-02$ $0.980503 E-03$ $0.467550 E-03$ $0.235782 \mathrm{E}-03$ . $122371 E-03$

$0.873181 E-03$ $0.372067 \mathrm{E}-03$ $0.166476 E-03$ $0.790241 E-04$ $0.388310 E-04$

$0.660179 \Xi-04$ $0.364157 \bar{E}-04$ $0.198863 E-04$ $0.104547 \bar{E}-04$ $0.566868 \bar{E}-05$ $0.301428 E-01$

$0.315182 E-00$ $0.366803 E-01$ $0.743820 \varepsilon-02$ $0.178563 \varepsilon-02$ $0.535598 \mathrm{E}-03$

$0.174199 E-03$ $0.642378 E-04$ $0.248902 E-04$ $0.104604 E-04$ $0.455083 E-05$

$0.209654 E-05$ $0.991492 E-06$ $0.489400 E-06$

$0.331791 E-00$ $0.409593 E-01$ $0.860064 E-02$ $0.214718 \mathrm{E}-02$ $0.660304 E-03$

$0.221027 E-03$ $0.831215 \mathrm{E}-04$ $0.329433 E-04$ $0.140727 E-04$ $0.623712 E-05$

$0.291407 \mathrm{E}-05$ $0.139998 E-05$ $0.699625 E-06$ MEV $=4.00$ $z=29$.

$0.149518 \equiv 01$ 0.376583 E- 00 $0.141857 E-00$ $0.590544 \varepsilon-01$ $0.277876 \mathrm{E}-0 \mathrm{I}$

$0.137946 \bar{E}-01$ $0.728703 \varepsilon-02$ $0.398612 \bar{E}-02$ C. $226662 \varepsilon-02$

$0.132163 E-02$

$0.791803 E=03$ $0.483568 \bar{E}-03$ $0.301405 E-03$

$0.789790 E \quad 00$ 0.17275 CE-00 $0.561377 E-01$ $0.209535 \varepsilon-01$ $0.891719 E-02$

$0.400896 E-02$ $0.198967 E-02$ 0.101640 E-02 $0.542470 E-0_{3}$ $0.296541 E-03$

$0.169442 E-03$ $0.984120 E-04$ $0.585002 \bar{E}-04$

$0.577916 E 00$ $0.114444 E=00$ $0.336913 E-01$ $0.116325 E=01$ $0.460396 E-02$

$0.197291 \bar{E}-02$ $0.910123 E-03$ $0.441124 \bar{E}-03$ $0.224140 \varepsilon-03$ $0.117877 \varepsilon-03$

$0.640970 E-04$ $0.357624 E=04$ $0.204628 E-04$ $z=29$.

O $0 \quad 0.1283708 \quad 01$ $0.418644 E=00$ $0.180140 \bar{E}-00$ $0.670162 \mathrm{E}-0$ 0.45781 E-0I

$0.254550 E-01$ $0.148127 E-0$ $0.891035 E-02$ $0.552685 E-02$ $0.350698 \varepsilon-02$

$0.227241 E-02$ $0.149844 E-02$ $0.100408 \mathrm{E}-02$
$0.860967 \bar{E} 00$ $0.24818 \equiv E-00$ $0.964691 \bar{E}-01$ $0.428992 E-01$ $0.209860 E-01$

$0.104450 E-01$ $0.601090 \bar{E}-02$ $0.343290 \bar{E}-02$ $0.202721 E-02$ ऽ. $122587 E-02$

$0.764162 E-03$ $0.484450 E-03$ C. $312799 E-03$
$0.700630 E 00$ $0.186629 E=00$ $0.679755 \varepsilon-01$ $0.266279 E-01$ $0.133397 \varepsilon-01$

$0.6660565-02$ $0.351476 E-02$ 0. $193493 E-02$ $0.110417 \varepsilon-02$ $0.048771 \varepsilon-03$

$0.391123 E-03$ $0.240987 \bar{E}-03$ $0.151446 \bar{E}-03$
$0.382497 \mathrm{E}-00$ $0.557530 E-01$ $0.130027 E-01$ $0.362522 \mathrm{E}-02$ $0.120409 E-02$

$0.437418 E-03$ $0.174976 \mathrm{E}-03$ $0.740229 \mathrm{E}-04$ $0.333123 E-04$ C. $155909 E-04$

$0.762200 E-05$ $0.383770 E-05$ $0.199660 \mathrm{E}-05$

$0.463916 E-00$ 0.845172 E-0I $0.229764 \mathrm{E}-0 \mathrm{I}$ $0.743410 \mathrm{E}-02$ $0.276938 E-02$

$0.112514 E-02$ $0.493893 E-03$ $0.228801 E-03$ $0.111423 E-03$ $0.563312 E-04$

$0.295077 E-04$

$0.248745 E-00$ $0.263397 \vec{E}-01$ $0.478232 E-02$ $0.105633 E-02$ $0.290590 E-03$

$0.880385 E-04$ $0.302539 E-04$ $0.110299 E-04$ $0.436707 E-05$ $0.180141 E-05$

$0.787976 E-06$ $0.355426 E-06$ $0.167557 E-06$

$0.263926 E-00$ $0.297185 E-01$ $0.562251 E-02$ $0.129582 E-02$ $0.367316 E-03$

$0.114884 E-03$ $0.404197 E-04$ $0.151132 E-04$ $0.610140 E-05$ $0.256987 E-05$

$0.114297 E-05$ $0.524771 E-06$ $0.251008 E-06$ 
Table 2. Spatial Moments, Plane Perpendicular Source-Continued

$0.211954 E \quad 01$ $0.235416 E-00$ $0.815438 \mathrm{E}-01$ $0.206691 \mathrm{E}-01$ $0.864823 E-02$ $0.637948 E-01$ $0.164232 E-01$ $0.372254 E-02$ $0.130823 E-02$

$0.298817 E-02$ $0.139028 \mathrm{E}-02$ $0.570350 \mathrm{E}-03$ $0.285624 \mathrm{E}-03$ $0.131006 E-03$

$0.412418 E-03$ $0.169983 E-03$ $0.645869 E-04$ $0.294815 E-04$

$0.126619 E-04$

$0.693742 E-04$ $0.344513 E-04$

$0.621967 E-05$

$0.291617 E-05$

$0.151552 E-05$

$0.371104 E-00$ $0.330460 E-01$ $0.684021 \mathrm{E}-02$ $0.139850 E-02$

$0.122684 E-03$ $0.453723 E-04$ $0.160209 E-04$ $0.671315 E-05$ $0.270625 E-05$

$0.123853 E-05$ $0.549239 E-06$ $0.268707 E-06$ MEV $=0.10$

$0.215043 E-00$

$0.716962 \mathrm{E}-01$

$0.172138 E-01$

$0.692665 E-02$

$0.228122 E-02$

$0.102035 E-02$

$0.400554 E-03$

$0.192885 E-03$

$0.848891 \mathrm{E}-04$

$0.432491 E-04$

$0.206497 \mathrm{E}-04$

$0.110075 E-04$

$z=50$.
$0.653575 E 00$

$0.613067 E-01$

$0.152845 E-01$

$0.330374 E-02$

$0.112116 \mathrm{E}-02$

$0.338451 E-03$

$0.134489 \mathrm{E}-03$

$0.490581 E-04$

$0.215788 E-04$

$0.891400 E-05$

$0.421981 E-05$

$0.190575 E-05$

$0.954845 E-06$ $z=50$.

$0.201897 E$ OI $0.205269 \mathrm{E}-00$ $0.666747 E-01$ $0.156733 E-01$ $0.614824 E-02$

$0.198453 E-02$ $0.865731 E=03$

$0.333278 E-03$ $0.156613 E=03$ $0.676175 E-04$

$0.336375 E-0.4$ $0.157601 E=04$ $0.820766 E-05$

$0.662898 E 00$ $0.610985 E=01$ $0.149597 \mathrm{E}-0$ $0.317794 E-02$ $0.105628 E-02$

$0.313343 E-03$ $0.121807 E-03$

$0.436546 E-04$ $0.187789 E-04$ $0.762095 \mathrm{E}-05$

$0.352843 E-05$

$0.156544 E-05$

$0.767291 E-06$ $Z=50$.

$0.196068 E$ OI $0.204177 \mathrm{E}=00$ $0.650598 E-01$ $0.155116 \mathrm{E}-01$ $0.598905 E-02$

$0.194989 E-02$ $0.838945 E-03$ $0.324582 E-03$ $0.150637 \mathrm{E}-03$ $0.651971 \mathrm{E}-04$

$0.320623 E-04$ $0.150313 E=04$ $0.774398 E-05$ $M E V=0.70$

- 0 0.188653E 01 $0.209573 E-00$ $0.658805 E-01$ $0.658805 E=01$ $0.628417 E-02$

$0.211121 E-02$ $0.904831 E-03$ $0.358585 \mathrm{E}-03$ $0.166118 E-03$ $0.732789 E-04$

$0.360169 E-04$ $0.171487 E-04$ $0.883675 E-05$
$0.674639 E \quad 00$ $0.638034 E-01$ $0.155235 E-0.1$ $0.334919 E-02$ $0.110327 E-02$

$0.330466 E-03$ $0.127244 E-03$ $0.458694 \mathrm{E}-04$ $0.195444 E-04$ $0.795661 \mathrm{E}-05$

$0.364950 E-05$ $0.162110 E-05$ $0.787323 E-06$ $2=50$

$0.410328 E-00$ $0.355919 E-01$ $0.713892 \mathrm{E}-02$ $0.140991 E-02$ $0.408327 E-03$

$0.113425 E-03$ $0.397003 E-04$ $0.134133 E-04$ $0.529767 \mathrm{E}-05$ $0.203783 E-05$

$0.877453 E-06$ $0.170484 E-06$

$0.690286 E \quad 00$ $0.696077 E-01$ $0.696077 \mathrm{E}=01$ $0.170208 E-01$ $0.126567 \mathrm{E}-02$

$0.391314 E-03$ $0.150976 E-03$ $0.557725 \mathrm{E}-04$ $0.238155 E-04$ $0.988654 \mathrm{E}-05$

$0.454514 E-05$ $0.205146 E-05$ $0.998685 E-06$

$0.431057 E-00$ $0.399547 E-01$ $0.818054 \mathrm{E}-02$ $0.495752 \mathrm{E}-03$

$0.142474 \mathrm{E}-03$ $0.503065 E-04$ $0.174530 E-04$ $0.694183 E-05$

$0.118156 E-05$ $0.507887 E-06$ $0.234854 \mathrm{E}-06$ $0.272770 \mathrm{E}-05$ $0.424379 E-03$
$0.383376 E-00$ $0.326594 E-01$ $0.658869 \mathrm{E}-02$ $0.129371 \mathrm{E}-02$

$0.381048 E-03$

$0.106042 E-03$ $0.379585 E-04$ $0.129177 E-04$ $0.523181 E-05$

$0.203461 E-05$

$0.899540 E-06$ $0.385144 E-06$ $0.182020 E-06$
$0.395172 E-00$ $0.332872 \mathrm{E}-01$ $0.664435 \mathrm{E}-02$ $0.128812 E-02$ $0.373615 E-03$

$0.102531 E-03$ $0.360451 E-04$ $0.120839 E=04$ $0.480027 E-05$ $0.183773 E-05$

$0.796462 E-06$ $0.335555 E-06$ $0.155424 E-06$ $0.370623 E-06$

$0.259326 E-00$ $0.209325 E-0$ $0.365803 E-02$ $0.680999 E-0$ $0.182391 \mathrm{E}-03$

$0.486437 E-04$ $0.163292 E-04$ $0.538011 E-05$ $0.208381 E-0$ $0.790811 \mathrm{E}=06$

$0.338723 E-06$ $0.142391 E-06$ $0.657872 \mathrm{E}-07$

$0.270443 E-00$ $0.210845 E-0$ $0.360913 E-02$ $0.049382 \mathrm{E}-03$

$0.169615 E-03$

$0.437678 E-04$ $0.142780 E-04$ $0.455198 \mathrm{E}-05$ $0.170966 \mathrm{E}-05$ $0.627880 E-06$

$0.260501 E-06$ $0.105997 E-00^{\circ}$ $0.474131 \mathrm{E}-07$

$0.281254 \mathrm{E}=00$ $0.218090 E-01$ $0.371445 \mathrm{E}-02$ $0.662748 \mathrm{E}-03$ $0.171296 E-03$

$0.437438 \mathrm{E}-04$ $0.140692 E-04$ $0.443109 \mathrm{E}-0$ $0.163736 E-0$ $0.593312 E=06$

$0.241899 E-06$ $0.970339 E-07$ $0.426257 \mathrm{E}-07$

$0.295324 \mathrm{E}-00$ $0.236670 E=01$ $0.408446 \mathrm{E}-02$ $0.744853 \mathrm{E}-03$ $0.193489 \mathrm{E}-03$

$0.501562 E-04$ $0.161404 E-04$ $0.513525 E-0$ $0.189380 \mathrm{E}-0$ $0.690872 E-06$

$0.280692 E-06$ $0.113073 E-06$ $0.494502 E-07$ $0.169110 E-02$

$0.198934 E-00$ $0.146746 E-01$ $0.223484 E-02$ $0.382068 E-03$ $0.918464 E-04$

$0.227293 E-04$ $0.699312 E-05$ $0.215868 E-05$ $0.777598 E-06$ $0.278640 E-06$

$0.112170 E-06$ $0.448011 E-07$ $0.196068 \mathrm{E}-07$

$0.208691 E-00$ $0.149928 E-01$ 
Table 2. Spatiol Moments, Plone Perpendicular Source-Continued

n

$$
p=-1 / 2
$$$$
1 / 2
$$

$3 / 2$

$5 / 2$

$7 / 2$

$9 / 2$

$11 / 2$ $0.759749 E-01$ $0.852592 \mathrm{E}-04$

$0.166229 \mathrm{E} \mathrm{OI}$
$0.182016 E 01$

$0.705028 E 00$ $0.187673 E-01$ $0.441848 E-02$ $0.147006 \mathrm{E}-02$ $0.175624 E-01$ $0.672288 E-02$

$0.233309 \mathrm{E}-02$ $0.100198 \mathrm{E}-02$ $0.407229 \mathrm{E}-03$ $0.189366 \mathrm{E}-03$

$0.469695 E-03$ $0.182575 \mathrm{E}=03$ $0.692098 E-04$ $0.297735 E-04$ $0.126232 E-04$

$0.421024 E-04$ $0.203918 E-04$

$0.584587 E-05$ $0.268572 E-05$ $0.105623 \mathrm{E}-04$ $0.131682 E-05$ $z=50$. $0.239573 E-00$ $0.750472 E-01$ $0.220783 E-01$ $0.860304 \mathrm{E}-02$

$0.326758 E-02$ $0.143778 E=02$ $0.627508 E-03$ $0.299775 E-03$ $0.143178 E-03$

$0.743289 E$ OO $0.968341 E-01$ $0.250330 E-01$ $0.667265 E-02$ $0.230878 E-02$

$0.451152 E-00$ $0.447614 \mathrm{E}=01$ $0.938938 E-02$ $0.203380 E-0$ $0.606190 E-03$

$0.180437 E-03$ $0.645765 E-04$ $0.230354 E-04$ $0.927092 E-05$ $0.372704 \mathrm{E}-05$

$0.163172 E-05$ $0.715037 E-06$ $0.333890 E-06$

$0.809187 E-03$ $0.326514 E=03$ $0.133259 \mathrm{E}-03$ $0.594201 E-04$ $0.267931 \mathrm{E}-04$

$0.727000 E-04$ $0.370406 E-04$

$0.128411 E-04$ $0.315160 E-05$ $0.217351 E-04$ $0.622080 E-05$
$0.505939 E \quad 00$ $0.608830 E=01$ $0.138126 E-01$ $0.340934 \mathrm{E}-02$ $0.107869 E-02$

$0.354403 E-03$ $0.133521 E-03$ $0.515694 E-04$ $0.933993 E-05$

$0.426562 E-05$ $0.197989 E-05$ $0.961416 E-06$
$0.334208 E-00$ $0.307823 E-01$ $0.568044 \mathrm{E}-02$ $0.114304 \mathrm{E}-0$ $0.310589 E-03$

$0.867562 E-04$ $0.288898 E-04$ $0.975259 \bar{E}-05$ $0.369861 \mathrm{E}-0$ $0.141629 E-05$

$0.589308 E-06$ $0.247236 E-06$ 0.110405 E-06

$0.388619 E-00$ $0.436085 E-01$ $0.892260 E-02$ $0.206088 \mathrm{E}-02$ $0.604927 E-03$

$0.187812 E-03$ $0.666869 E=04$ $0.245288 E-04$ $0.983870 E-05$ $0.404991 E-05$

$0.177193 E-05$ $0.791379 E-06$ $0.369923 E=06$ $Z=50$.

$$
\text { MEV }=4.00
$$
$0.874 \mathrm{C} 41 \mathrm{E}=0$

$0.476666 \mathrm{E}-00$ $0.698177 E=01$ $0.166827 \mathrm{E}=01$ $0.461884 \mathrm{E}-0$
$0.797057 E \quad 00$ $0.133502 E-00$ $0.377488 E-01$ $0.117744 E-0$. $0.440065 E-02$ $0.888482 \mathrm{E}-01$ $0.303306 \mathrm{E}-01$ $0.124397 \mathrm{E}=01$

$0.526459 E-02$ $0.244442 E-02$ $0.116332 \mathrm{E}-02$ $0.585945 E-03$ $0.301124 \mathrm{E}-03$

$0.173289 E-02$ $0.750038 E-03$ $0.336296 E-03$ $0.159954 E-03$ $0.781168 E-04$

$0.397454 E-04$ $0.200493 E=04$

$0.160883 E-0$

$0.110602 E-04$

$0.589712 E \quad 00$ $0.918895 E-01$ $0.236636 E=01$ $0.691871 \mathrm{E}=02$ $0.242025 E-02$

$0.903447 E-03$ $0.371146 E-03$ $0.159038 E-03$ $0.724046 E-0$ $0.339902 E-04$

$0.166478 E-04$ $0.835013 E-05$ $0.432326 E-05$ $z=50$.

$0.125340 E$ OI $0.327278 \mathrm{E}-00$ $0.121946 E-00$ $0.508175 E-01$ $0.236335 E-01$

$0.116337 \mathrm{E}-01$ $0.606391 \mathrm{E}-02$ $0.327652 \mathrm{E}-02$ $0.183605 E-02$ $0.105520 E-02$

$0.622816 \mathrm{E}-03$ $0.374914 E-03$

MEV $=0.10$

$0.872687 E \quad 00$ $0.208403 E-00$ $0.711676 E-01$ $0.277170 E-01$ $0.121182 E-01$

$0.565588 E-02$ $0.280738 \mathrm{E}-02$ $0.145156 \mathrm{E}-02$ $0.780768 E-03$ $0.432222 \mathrm{E}-03$

$0.246178 E-03$ $0.143399 E-03$

$0.722447 \mathrm{E} \quad 00$ $0.162842 E-00$ $0.526326 E-01$ $0.196126 E-01$ $0.823256 \mathrm{E}-02$

$0.370732 E-02$ $0.178013 \mathrm{E}-02$ $0.893005 E-03$ $0.466905 E-03$ $0.251752 E-03$

$0.139872 E-03$ $0.796084 \mathrm{E}-0$ $0.464375 E-04$

$Z=82$.

$0.215598 E$ Ol $0.145450 E=00$ $0.810198 \mathrm{E}-02$ $0.313144 E-02$

$0.773991 E=03$ $0.328149 E-03$ $0.100658 E-03$ $0.455790 E-04$ $0.161539 E-04$

$10 \quad 0.770234 E=05$

$110.303136 E-05$ $0.150822 E-05$
$0.637042 E \quad 00$ $0.399781 \mathrm{E}-01$ $0.945426 E-02$ $0.148787 \mathrm{E}-02$ $0.470640 E-03$

$0.110047 E-03$ $0.410248 E-04$ $0.118577 \mathrm{E}-04$ $0.487253 E=05$ $0.163826 E-05$

$0.722688 E-06$ $0.271285 E-06$ $0.126499 E-06$
$0.362973 E-00$ $0.212012 E-01$ $0.394269 E-02$ $0.574647 \mathrm{E}-03$

$0.338981 \mathrm{E}-04$ $0.112572 \mathrm{E}-04$ $0.306801 E-05$ $0.115210 E-05$ $0.367667 E-06$

$0.150683 E-06$ $0.539713 E-07$ $0.236501 E=07$ $0.157015 E-03$

$0.266149 E-00$ $0.228941 E-01$ $0.379942 \mathrm{E}-02$ $0.715244 E-03$

$0.179310 E-03$

$0.472562 E-04$ $0.147534 \mathrm{E}-04$ $0.473339 E-05$ $0.170061 E-05$ $0.622436 E-06$

$0.247143 E-06$ $0.995533 E=07$ $0.426501 E-07$

$0.221403 E-00$ $0.178731 E=01$ $0.270839 \mathrm{E}-02$ $0.479632 E-03$ $0.111932 \mathrm{E}-03$

$0.279522 E-04$ $0.822968 E-05$ $0.251802 E-05$ $0.860687 E-06$ $0.301953 E-06$

$0.124781 E-06$ $0.444984 E-07$ $0.183370 E-07$

$0.317090 E-00$ $0.333873 \mathrm{E}=01$ $0.626340 \mathrm{E}-02$ $0.136316 \mathrm{E}-02$ $0.374710 E-03$

$0.110529 E-03$ $0.372207 \mathrm{E}=04$ $0.130945 E-0$ $0.502193 \mathrm{E}-0$ $0.198726 \mathrm{E}-05$

$0.836035 E-06$ $0.360375 E-06$ $0.162654 E-06$ 
Table 2. Spatial Moments, Plane Perpendicular Source-Continued

n

$p=-1 / 2$

$1 / 2$

$3 / 2$

$5 / 2$

$7 / 2$

$9 / 2$

$11 / 2$

\begin{tabular}{|c|c|c|}
\hline 0 & $0.208503 \mathrm{E} \mathrm{O1}$ & $0.650141 E 00$ \\
\hline 1 & $0.129397 E-00$ & $0.378541 \mathrm{E}-01$ \\
\hline 2 & $0.405122 E-01$ & $0.866059 \mathrm{E}-02$ \\
\hline 3 & $0.640491 E-02$ & $0.127430 E-02$ \\
\hline 4 & $0.236493 E-02$ & $0.392324 E-03$ \\
\hline 5 & $0.546094 E-03$ & $0.850667 E-04$ \\
\hline 6 & $0.220857 \mathrm{E}-03$ & $0.303625 E-04$ \\
\hline 7 & $0.635871 \mathrm{E}-04$ & $0.826954 E-05$ \\
\hline 8 & $0.274445 E-04$ & $0.324725 E-05$ \\
\hline 9 & $0.916256 E-05$ & $0.103163 E-05$ \\
\hline 10 & $0.416290 \mathrm{E}-05$ & \\
\hline 11 & $0.154778 E-05$ & $0.154459 \mathrm{E}-06$ \\
\hline
\end{tabular}

$0.733784 E-06$
$0.379085 E-00$ $0.207433 E-01$ $0.377005 E-02$ $0.517870 E-03$ $0.137012 \mathrm{E}-03$

$0.279536 E-04$ $0.893498 E-05$ 0.230574 E-05 $0.830644 E-06$ $0.251432 E-06$

$0.986783 E-07$ $0.335769 E-07$ $0.140762 E-07$ $2=82$ MEV $=0.40$

$0.666398 E \quad 00$ $0.386056 E-01$
$0.865618 E-02$ $0.126244 E-02$ $0.378532 E-03$ $0.370955 E-01$ $0.579388 E-02$ $0.206347 E-02$

$0.811198 E-04$ $0.281352 E-04$ $0.755804 E-05$ $0.288196 E-05$ $0.901714 E-06$

$0.183513 E=0$

$0.520154 E-04$

$0.217093 E-04$
$0.712748 E-05$

$0.313455 E-05$

$0.114513 E-05$

$0.368745 E-06$

$0.128961 E-06$ $0.557353 E-07$

MEV $=0.70$

$0.190429 E 01$ $0.124255 E=00$ $0.362491 E-01$ $0.591669 E-02$ 0.205293 E-02

$0.483367 E-03$ $0.184656 E-03$ $0.537625 E-04$ $0.220120 E-04$ $0.738256 E-05$

$0.319240 E-05$ $0.118630 \mathrm{E}-05$ $0.536695 E-06$

\section{$0.686462 E 00$ $0.422318 E-01$ $0.939300 E=02$ $0.143238 \mathrm{E}-02$ $0.423803 E-03$}

$0.940092 E-04$ $0.321566 E-04$ $0.887634 E-05$ $0.333989 E-05$ $0.106786 E-05$

$0.431261 E-06$ $0.153472 E-06$ $0.655528 E-07$
$0.399654 E-00$ $0.218830 E-01$ $0.396087 \mathrm{E}-02$ $0.542024 E-03$ $0.141196 E=03$

$0.285853 E-04$ $0.894626 E-05$ $0.228426 E-05$ $0.803549 E-06$ $0.240162 E-06$

$0.919126 E-07$ $0.308329 E-07$ $0.125961 \mathrm{E}-07$ $z=82$
$0.425935 E-00$ $0.248427 \mathrm{E}=01$ $0.455604 E-02$ $0.653853 E-03$ $0.170447 \mathrm{E}=03$

$0.358066 E-04$ $0.111604 E-04$ $0.293419 E-05$ $0.102589 E-05$ $0.313892 E-06$

\section{$0.119294 E-06$} $0.407845 E-07$ $0.165393 E-07$

$0.266540 E-00$ 0.349600 E-O5 $0.856624 E-06$ $0.824235 E-07$

$$
M E V=1.00 \quad .2=82
$$

$\begin{array}{llll}0 & 0.181865 E-1 & 0.705370 E-00 & 0.451625 E-00 \\ 1 & 0.128040 E-00 & 0.468159 E-01 & 0.284706 E-01 \\ 2 & 0.365076 E-01 & 0.104137 E-01 & 0.532366 E-02 \\ 3 & 0.631034 E-02 & 0.168145 E-02 & 0.810674 E-03 \\ 4 & 0.215368 E-02 & 0.495763 E-03 & 0.213264 E-03 \\ 5 & 0.530928 E-03 & 0.115085 E-03 & 0.469732 E-04 \\ 6 & 0.200546 E-03 & 0.392370 E-04 & 0.147224 E-04 \\ 7 & 0.605889 E-04 & 0.112404 E-04 & 0.402398 E-05 \\ 8 & 0.246079 E-04 & 0.421917 E-05 & 0.141273 E-05 \\ 9 & 0.851047 E-05 & 0.139141 E-05 & 0.446534 E-06 \\ 0 & 0.365895 E-05 & 0.561020 E-06 & 0.170295 E-06 \\ 1 & 0.139535 E-05 & 0.204950 E-06 & 0.598519 E-07 \\ 2 & 0.628667 E-06 & 0.874612 E-07 & 0.243484 E-07\end{array}$

$$
M E V=2.00 \quad Z=82 \text {. }
$$

$0.165215 E$ OI $0.146382 E-00$ $0.408400 E-01$ $0.637800 E-02$

$0.284931 E-02$

$0.805298 E-03$ $0.306551 E-03$ $0.103708 E-03$ $0.427448 E-04$ $0.162757 E-04$

$0.713232 E-05$ $0.295694 \mathrm{E}-05$ $0.136165 \mathrm{E}-05$
$0.745919 E \quad 00$ $0.620839 E-01$ $0.141726 E-01$ $0.271018 E-02$ $0.817193 E-03$

$0.217301 E-03$ $0.759179 E-04$ $0.243530 E-04$ $0.938565 \mathrm{E}-05$ $0.340932 E-05$

$0.141335 E-05$ $0.561719 E-06$ $0.246650 E-06$
$0.509848 E 00$ $0.403520 E-01$ $0.804347 \mathrm{E}-02$ $0.145459 E-02$ $0.401014 E-03$

$0.101487 E-03$ $0.331570 E=04$ $0.101797 E-04$ $0.371664 E-05$

$0.514022 E-06$ $0.197126 E-06$ 0.831772 E-07 $0.129795 E-05$ $0.201156 E-01$ $0.874139 \mathrm{E}=08$ $0.137474 E-01$ $0.209057 \mathrm{E}-02$ $0.269181 E-03$ $0.626458 E-04$

$0.120603 E-04$ $0.285307 \mathrm{E}-06$

$0.302989 E-07$ $0.988146 E=08$ $0.391646 \mathrm{E}-08$

$0.285394 E-00$ $0.148141 E-01$ $0.227020 E-02$ $0.292742 E-03$ $0.677305 E-04$

$0.129961 E-04$ $0.371592 E-05$ $0.904164 E=06$ $0.295743 \mathrm{E}=06$ $0.846157 \mathrm{E}-07$

$0.304704 E-07$ $0.982206 \mathrm{E}-08$ $0.380780 \mathrm{E}-08$

$0.310020 E-00$ $0.172010 E-01$ $0.271366 \mathrm{E}-02$ $0.368301 E-03$ $0.863191 \mathrm{E}=04$

$0.172408 E-04$ $0.495474 E-05$ $0.124478 \mathrm{E}-05$ $0.407568 \mathrm{E}-06$

$0.119663 E-06$

$0.430348 E-07$ $0.141677 \mathrm{E}-07$ $0.547754 E-08$

$0.205256 E-00$ $0.100086 E-01$ $0.131774 \mathrm{E}-02$ $0.159681 E-03$ $0.332769 E=04$

$0.606263 E-05$ $0.161048 E-05$ $0.375535 E-06$ $0.116413 E-06$ $0.321552 E-07$

$0.111226 E-07$ $0.348186 \mathrm{E}-08$ $0.130908 E-08$

$0.221937 E-00$ $0.109507 E-01$ $0.146553 E-02$ $0.178748 E-03$ $0.373337 E-04$

$0.680938 E-05$ $0.179621 E-05$ $0.417488 E-06$ $0.127797 E-06$ $0.350715 E-07$

$0.119378 \mathrm{E}-07$ $0.370384 E-0 B$ $0.136725 E-08$

$0.244060 E-00$ $0.129161 E-01$ $0.180096 E-02$ $0.232044 \mathrm{E}-03$ $0.496027 E-04$

$0.944918 E-05$ $0.252608 E-05$ $0.60 B 026 E-06$ $0.187560 E-06$ $0.529605 E-07$

$0.181025 E-07$ $0.574981 E-08$ $0.212647 E-08$

$0.334668 E-00$ $0.328317 E-02$ $0.474076 \mathrm{E}-03$

$0.123353 E-03$

$0.237965 E-04$ $0.693319 E-05$ $0.181487 \mathrm{E}-05$ $0.600478 E-06$

$0.182498 \mathrm{E}-06$

$0.661961 E-07$ $0.224449 E-07$

$0.392598 E-00$ $0.296927 \mathrm{E}-01$ $0.531817 \mathrm{E}-02$ $0.915541 E-03$

$0.234226 \mathrm{E}-03$

$0.567293 E-04$ $0.174973 E-04$ $0.516554 E-05$ $0.179883 \mathrm{E}-05$ $0.606382 E-06$

$0.230615 E-06$ $0.856344 \mathrm{E}-07$ $0.348690 E-07$

$0.166792 E-00$ $0.771089 E-02$ $0.900110 E-03$ $0.103034 \mathrm{E}-03$

$0.194941 E-04$

$0.337048 E-05$ $0.827566 E-06$ $0.184045 E-06$ $0.534094 E-07$ $0.141301 E-07$

$0.461811 E-08$ $0.138967 E-08$ $0.497125 E-09$

$0.181564 E-00$ $0.853667 E-02$ $0.101951 E-02$ $0.118009 E-03$ $0.225291 E-04$

$0.391629 E-05$ 
Table 2. Spatial Moments, Plane Perpendicular Source-Continued

MEV $=4.00$

$z=82$.

$0.144942 E \quad 01$ $0.175290 E=00$ $0.488544 E=0$ $0.124684 \mathrm{E}-0$ $0.432734 \mathrm{E}-02$

$0.144980 E-02$ $0.569255 \mathrm{E}-03$ $0.221469 \mathrm{E}-03$ $0.946449 E-04$ $0.405929 E-04$

$0.184882 \mathrm{E}-04$ $0.850554 E-05$

$0.407434 \mathrm{E}-05$
0.803859 E 00 $0.911787 \mathrm{E}-01$ $0.220284 \mathrm{E}-01$ $0.525701 E-02$ $0.166951 \mathrm{E}-02$

$0.528464 E-03$ $0.194395 E-03$ $0.720346 E-04$ $0.292140 E-04$

$0.523197 E-05$ $0.231722 \mathrm{E}-05$

$0.106803 E-05$ $0.120066 \mathrm{E}=04$ Air $0.718231 E$ OO $0.357447 \mathrm{E}-00$ $0.193674 \mathrm{E}-00$ $0.115974 \mathrm{E}=00$

$0.723178 \mathrm{E}-01$ $0.474855 \mathrm{E}-01$ $0.319594 \mathrm{E}-01$ $0.222188 E-01$ $0.157111 E=0$

$0.113640 \mathrm{E}-01$ $0.832331 \mathrm{E}-02$ $0.620031 E-02$

$0.664624 \mathrm{E} \quad 00$ $0.174648 \mathrm{E}-00$ $0.675555 E-01$ $0.303174 E-01$ $0.154210 E-01$

$0.839313 E-02$ $0.488290 \mathrm{E}-02$ $0.295650 \mathrm{E}-02$ $0.186718 \mathrm{E}-02$ $0.121108 \mathrm{E}-02$

$0.808958 E-03$ $0.550847 \mathrm{E}-03$ $0.383374 E-03$

MEV $=0.05$

Air

0

$0.196193 E 01$ 0.705657 E 00 $0.348996 \mathrm{E}-00$ $0.188819 \mathrm{E}-00$ $0.112446 \mathrm{E}-00$

$0.698961 E-0$ $0.456573 \mathrm{E}-0$ $0.306120 E-01$ $0.211749 \mathrm{E}-0$ $0.149114 E-01$

$0.107323 E-01$ $0.782718 \mathrm{E}-0$ $0.580240 \mathrm{E}-02$

$0.674383 E 00$ $0.179575 E-00$ $0.695371 \mathrm{E}-0$ $0.312723 \mathrm{E}-01$ $0.158762 \mathrm{E}=01$

$0.863278 E-02$ $0.500728 E-02$ $0.302496 E-02$ $0.190367 E-02$ $0.123109 E-02$

$0.819174 \mathrm{E}-03$ $0.555917 \mathrm{E}-03$ $0.385352 \mathrm{E}-03$

MEV $=0.10$

Air

$0.197775 E$ O $0.721772 \mathrm{E} O 0$ $0.358245 \mathrm{E}-00$ $0.195105 E-00$ $0.116541 E=00$

$0.671151 E 00$ $0.180139 \mathrm{E}-00$ $0.698270 E-01$ $0.315178 E=01$ $0.160228 E-01$

$0.127640 \mathrm{E}-01$ $0.476509 \mathrm{E}=01$ $0.320552 E-01$ $0.222199 \mathrm{E}-0$ $0.156884 E-01$

$0.113115 E-01$ $0.826711 E-02$ $0.613760 E-02$

$0.873602 E-02$ $0.507490 E-02$ $0.307278 E=0$ $0.193642 \mathrm{E}-02$ $0.125465 \mathrm{E}-02$

$0.835869 E-03$ $0.568151 E-03$ $0.394253 \mathrm{E}-03$

MEV $=0.20$

Air

$0.196606 E$ O $0.726624 E 00$ $0.360996 E-00$ $0.197482 E=00$ $0.118047 \mathrm{E}-00$

$0.738788 E-0$ $0.484020 E-01$ $0.326056 E-01$ $0.226054 \mathrm{E}-01$ 0.159734 E-0I

$0.115164 E-01$ $0.842039 E-02$

$0.625008 E=02$
$0.673536 E \quad 00$ $0.182837 \mathrm{E}-00$ $0.710537 E-01$ $0.322106 E-01$ $0.163968 E-01$

$0.896200 E-02$ $0.520982 \mathrm{E}-02$ $0.315908 E-02$ $0.199159 E-02$ $0.129153 E-02$

$0.860546 E-03$ $0.585208 \mathrm{E}-03$ $0.406053 E-03$
$0.600905 E 00$ $0.650525 E-01$ $0.143264 \mathrm{E}-01$ $0.325720 \mathrm{E}-02$ $0.972066 \mathrm{E}-03$

$0.295044 E-03$ $0.103490 \mathrm{E}-03$ $0.369676 \mathrm{E}-04$ $0.144161 E=04$ $0.573452 E-05$

$0.241592 E-05$ $0.103891 E-05$ $0.464743 E-06$

$0.488873 E-00$ $0.508133 \mathrm{E}-01$ $0.103941 \mathrm{E}-01$ $0.226721 E-02$ $0.642564 E-03$

$0.188108 \mathrm{E}-03$ $0.633727 \mathrm{E}-04$ $0.219292 \mathrm{E}-04$ $0.826854 \mathrm{E}-05$ $0.319694 \mathrm{E}-05$

$0.130803 E-05$ $0.548163 E-06$ $0.238899 E-06$

$0.397379 E-00$ $0.825087 E=0$ $0.263011 E-01$ $0.101140 \mathrm{E}-01$ $0.448773 \mathrm{E}-02$

$0.217138 \mathrm{E}-02$ $0.113583 E-02$ $0.625594 E-03$ $0.362222 \mathrm{E}-03$ $0.217099 E-03$

$0.134760 E-03$ $0.857570 E-04$ $0.560159 E=04$

$0.409994 E=00$ $0.869395 E=01$ $0.279540 E-01$ $0.108252 \mathrm{E}-01$ $0.481587 \mathrm{E}-02$

$0.233574 \mathrm{E}-02$ $0.122192 E-02$ $0.673128 E-03$ $0.389250 E-03$ $0.233039 E=03$

$0.144350 E-03$ $0.916832 \mathrm{E}-04$ $0.597286 \mathrm{E}=04$

$0.405790 E-00$ $0.862902 \mathrm{E}-01$ $0.277014 \mathrm{E}=0$ $0.107372 \mathrm{E}-0$

$0.477434 \mathrm{E}-02$

$0.231779 E-02$ $0.121268 E-02$ $0.668713 \mathrm{E}-03$ $0.386849 E-0$ $0.231829 \mathrm{E}=03$

$0.143670 E-03$ $0.913331 E-04$ $0.595297 \mathrm{E}=04$

$0.408889 \mathrm{E}-00$ $0.879590 E-01$ $0.283556 E-01$ $0.110444 E-01$ $0.492170 E-02$

$0.239608 E-02$ $0.125532 E-02$ $0.693473 E-03$ $0.401501 E-03$ $0.240888 E-03$

$0.149351 E-03$ $0.950121 E-04$
$0.283291 E-00$ $0.486035 \mathrm{E}-0$

$0.132235 E-01$ $0.445910 E-02$ $0.175879 E-02$

$0.767127 E-03$ $0.364972 \mathrm{E}-03$ $0.184516 E-03$ $0.986955 E=04$ $0.550025 \mathrm{E}-04$

$0.318987 E-04$ $0.190574 \mathrm{E}-04$ $0.117299 E-0$

$0.295011 E-00$ $0.520278 \mathrm{E}-0$ $0.143694 \mathrm{E}-0$ $0.490269 E-02$

$0.194729 E-02$

$0.854352 E-03$ $0.407821 \mathrm{E}-0$ $0.206768 \mathrm{E}-0$ $0.110733 E-03$ $0.617733 \mathrm{E}-04$

$0.358209 E-0$ $0.213963 E-04$ $0.131558 E=04$

$0.291090 \mathrm{E}-00$ $0.512850 \mathrm{E}-01$ $0.141107 \mathrm{E}-01$ $0.480773 \mathrm{E}-0$ $0.190529 \mathrm{E}-02$

$0.835291 E-03$ $0.398236 E-03$ $0.201856 \mathrm{E}-03$ $0.108033 E=03$ $0.602680 E-04$

$0.349368 \mathrm{E}-04$ $0.208709 E-04$ $0.128308 E=04$

$0.293980 E-00$ $0.524210 E-01$ $0.145037 E-01$ $0.496906 \mathrm{E}-02$ 0.197527 E-02

$0.868875 E-03$ $0.415063 \mathrm{E}-03$ $0.210857 \mathrm{E}-03$ $0.112998 E-03$ $0.531336 \mathrm{E}-04$

$0.366285 E-04$ $0.219039 \mathrm{E}-04$ $0.134724 \mathrm{E}-04$

$0.415259 E=00$ $0.415816 E-01$ $0.798688 \mathrm{E}-02$ 
Table 2. Spatial Moments, Plone Perpendicular Source - Continued

n

$$
p=-1 / 2
$$$$
1 / 2
$$

Air

MEV $=0.40$
$0.193466 \mathrm{E} 01$

$0.729586 E$ OO

$0.364348 \mathrm{E}-00$

$0.201019 \mathrm{E}-00$

$0.120635 \mathrm{E}-00$

$0.759028 \mathrm{E}-01$

$0.498807 E-02$

$0.337314 E-01$

$0.234434 E-01$

$0.166148 E-01$

$0.120031 \mathrm{E}-01$

$0.879712 E-02$
$0.654068 E-02$

$M E V=0.70$

$0.680035 E 00$ $0.188407 E-00$ $0.739031 \mathrm{~L}-01$ $0.338395 \mathrm{E}=01$

$0.173285 E-01$

$0.953196 E-02$ $0.556471 \mathrm{E}-02$ $0.338977 \mathrm{E}-02$ $0.214395 \mathrm{E}-02$ $0.139521 E=02$

$0.932019 E-03$ $0.635579 E-03$
$0.441928 E-03$

Air

\begin{abstract}
O. 193466 E OI
$0.732494 \mathrm{E} \mathrm{OO}$

$0.365447 \mathrm{E}=00$

$0.201790 E-00$

$0.121007 E-00$

$0.761462 E-01$

$0.500052 \mathrm{E}-01$

$0.338087 \mathrm{E}-02$

$0.234800 E-01$

$0.166341 \mathrm{E}-01$

$0.120078 E-01$

$0.879600 \mathrm{E}-02$

$0.653464 E-02$

$0.188955 E-00$

$0.740506 \mathrm{E}-01$

$0.339162 E-01$

$0.173527 E-01$

$0.954376 E-02$ $0.556719 \mathrm{E}-02$ $0.339006 \mathrm{E}-02$

$0.214247 \mathrm{E}-02$

$0.139357 \mathrm{E}-02$

$0.930183 E-03$ $0.633960 E-03$

$0.440441 E-03$
\end{abstract}

$M E V=1.00$

$0.680035 E 00$

Air

0

0.182248 E OL

$0.722003 E$ OO

$0.368356 E=00$

$0.208128 \mathrm{E}-00$

$0.126889 E-00$

$0.823430 E-01$

$0.539961 E-01$

$0.369786 \mathrm{E}-01$

$0.259710 E-01$

$0.186006 E-01$

$0.135601 E-01$

$0.100288 E-01$

$0.751662 E-02$

MEV $=2.00$

0.704500 E 00

$0.206761 \mathrm{E}=00$ $0.841037 E-01$

$0.397848 E-01$

$0.208665 \mathrm{E}-01$

$0.127319 E-01$

$0.696998 \mathrm{E}-02$

$0.431607 E-02$

$0.276784 \mathrm{E}-02$

$0.182515 \mathrm{E}-02$

$0.123332 E-02$

$0.850431 E-03$
$0.597184 E-03$

Air

0

$\begin{array}{ll}0.166734 \mathrm{E} 01 & 0.741968 \mathrm{E} 00 \\ 0.700656 \mathrm{E} 00 & 0.235285 \mathrm{E}-00 \\ 0.370237 \mathrm{E}-00 & 0.101296 \mathrm{E}-00 \\ 0.216259 \mathrm{E}-00 & 0.502938 \mathrm{E}-01 \\ 0.135288 \mathrm{E}-00 & 0.274205 \mathrm{E}-01 \\ 0.886039 \mathrm{E}-01 & 0.159477 \mathrm{E}-01 \\ 0.601780 \mathrm{E}-01 & 0.975193 \mathrm{E}-02 \\ 0.420102 \mathrm{E}-01 & 0.619795 \mathrm{E}-02 \\ 0.300170 \mathrm{E}-01 & 0.406753 \mathrm{E}-02 \\ 0.218524 \mathrm{E}-01 & 0.273993 \mathrm{E}-02 \\ 0.161723 \mathrm{E}-01 & 0.188775 \mathrm{E}-02 \\ 0.121341 \mathrm{E}-01 & 0.132555 \mathrm{E}-02 \\ 0.921784 \mathrm{E}-02 & 0.946632 \mathrm{E}-03\end{array}$

$M E V=4.00$

Air

0

$0.148027 E$ O1
$0.663454 E 00$
$0.368655 E-00$
$0.225379 E-00$
$0.146480 E-00$
$0.992995 E-01$
$0.695327 E-01$
$0.499307 E-01$
$0.366088 E-01$
$0.273060 E-01$
$0.206703 E-01$
$0.158462 E-01$
$0.122844 E-01$

$0.794306 E$

$0.277876 E-00$

$0.129566 E-00$

$0.688764 E-01$

$0.397651 \mathrm{E}-\mathrm{OI}$

$0.243153 \mathrm{E}-01$

$0.155340 E=01$

$0.102700 E-01$

$0.698359 E-02$

$0.486066 \mathrm{E}-02$

$0.345122 \mathrm{E}-02$

$0.249246 E=02$
$0.182728 E-O 2$
$3 / 2$

$5 / 2$

$7 / 2$

$9 / 2$

$11 / 2$
$0.417408 \mathrm{E}-00$ $0.918987 \mathrm{E}=01$ $0.300292 \mathrm{E}=02$ $0.118444 \mathrm{E}=01$ $0.532376 \mathrm{E}=02$

$0.261331 E-02$ $0.137744 E-02$ $0.765483 E-03$ $0.445211 \mathrm{E}-03$

$0.268329 E-03$

$0.166958 E-03$ $0.106596 E-03$ $0.696892 \mathrm{E}-04$ $0.921103 \mathrm{E}-01$ $0.300728 E-01$ $0.118609 \mathrm{E}-01$ $0.532606 E-02$

$0.261346 E-02$ $0.137632 \mathrm{E}-02$ $0.764492 \mathrm{E}-03$ $0.444265 E-03$ $0.267608 \mathrm{E}-03$

$0.166371 E-03$ $0.106154 \mathrm{E}-03$ $0.693420 \mathrm{E}-04$ $0.106559 E=00$ $0.366584 E-01$ $0.151071 \mathrm{E}=01$ $0.702612 \mathrm{E}-02$

$0.355394 E-02$ $0.192002 \mathrm{E}=02$ $0.109110 \mathrm{E}-02$ $0.646823 \mathrm{E}=03$ $0.396790 \mathrm{E}-03$

$0.250745 E-03$ $0.162442 E-03$ $0.107594 \mathrm{E}-03$

$0.504011 E$ $0.131882 \mathrm{E}-00$ $0.490618 E=01$ $0.215901 E-01$ $0.105973 \mathrm{E}-01$

$0.561555 E-02$ $0.315733 \mathrm{E}=02$ $0.185908 \mathrm{E}-02$ $0.113731 E-02$ $0.717930 E-03$

$0.465613 E-03$ $0.308970 \vec{E}-03$ $0.209225 \mathrm{E}-03$ $0.174712 \mathrm{E}-00$ $0.723667 E-0$ $0.348872 E-01$ $0.185006 \mathrm{E}-01$

$0.104877 \mathrm{E}-01$ $0.625575 E-02$ $0.388379 \mathrm{E}-02$ $0.249177 \mathrm{E}-02$ $0.164293 \mathrm{E}-02$

$0.110886 \mathrm{E}-02$ $0.763562 \mathrm{E}-03$ $0.535135 \mathrm{E}-03$
$0.301965 E-00$ $0.552590 E-01$ $0.155520 E-01$ $0.540952 E-02$

$0.217449 E-02$

$0.966381 E-03$ $0.465338 E-03$ $0.238182 \mathrm{E}-03$ $0.128413 \mathrm{E}-03$

$0.721627 E-04$

$0.420654 E-04$ $0.252713 E-04$ $0.156030 E-04$

$0.301965 E-00$ $0.553644 E-01$ $0.155695 \mathrm{E}-01$ $0.542415 E-02$

$0.217410 E-02$

$0.965685 E=03$ $0.464566 E-03$ $0.237646 \mathrm{E}-03$ $0.128011 \mathrm{E}-03$ $0.718914 \mathrm{E}-04$

$0.418712 \mathrm{E}=04$ $0.251375 E-04$ $0.155070 E-04$

$0.333501 E-00$ $0.664098 \mathrm{E}-01$ $0.199286 \mathrm{E}-01$ $0.731864 \mathrm{E}-02$ $0.307374 \mathrm{E}-02$

$0.141920 \mathrm{E}-02$ $0.705661 \mathrm{E}-03$ $0.371712 E-03$ $0.205445 \mathrm{E}-03$

$0.128104 \mathrm{E}-03$

$0.702427 E-04$ $0.429937 E-0.4$ $0.269941 \mathrm{E}=04$

$0.386662 E-00$ $0.868731 \mathrm{E}-0$ $0.287047 \mathrm{E}-0$ $0.114320 E=01$ $0.513855 E-02$

$0.251615 \mathrm{E}-02$ $0.131641 E-02$ $0.725489 \mathrm{E}-03$ $0.417424 \mathrm{E}-03$ $0.248871 \mathrm{E}-03$

$0.152992 \mathrm{E}-03$ $0.965366 \mathrm{E}-04$ $0.623344 E-04$

$0.471801 E=00$ $0.124337 \mathrm{E}=00$ $0.468175 \mathrm{E}-01$ $0.208389 E=01$ $0.103028 E-01$

$0.548393 E-02$ $0.308807 E-02$ $0.181797 E-02$ $0.111008 E-02$ $0.698801 E=03$

$0.451516 E-03$ $0.298360 \mathrm{E}-03$ $0.201082 \mathrm{E}-03$

$0.236783 \mathrm{E}-00$ $0.371037 \mathrm{E}-01$ $0.919460 \mathrm{E}-02$ $0.286724 \mathrm{E}-02$

$0.104482 E-02$

$0.424984 E-03$ $0.188663 \mathrm{E}-03$ $0.896165 E-04$ $0.450780 E-04$ $0.237503 E-04$

$0.130331 E=04$ $0.739848 E-05$ $0.433012 \mathrm{E}-05$

$0.236783 E=00$ $0.371642 E-01$ $0.920289 E-02$ $0.286867 E-02$

$0.104420 \mathrm{E}-02$

$0.424450 E-03$ $0.188237 E-03$ $0.893530 E-04$ $0.449039 E-04$ $0.236420 E-04$

$0.129621 E-04$ $0.735290 E-05$ $0.429964 E-05$

$0.265498 E-00$ $0.457469 E-01$ $0.122159 E-01$

$0.405930 E-02$ $0.155907 E-02$

$0.663947 E-03$ $0.306528 \mathrm{E}-03$ $0.150793 E-03$ $0.782093 \mathrm{E}-04$ $0.423712 \mathrm{E}-04$

$0.238363 E-04$ $0.138452 E-04$ $0.827305 E-05$

$0.315229 E-00$ $0.623038 \mathrm{E}-02$ $0.185996 \mathrm{E}-01$ $0.679401 E-02$ 
Table 2. Spatial Moments, Plane Perpendicular Source-Continued

$M E V=10.00$

Air

$0.124042 E \quad 01$ $0.589641 E 00$ $0.350544 E-00$ $0.22875 \mathrm{CE}=00$ $0.157693 E=00$

$0.112824 E-00$ C. $829849 E-01$ $0.023676 \mathrm{E}-01$ $0.477036 E-01$ $0.370230 E-01$

$0.291107 E-01$ $0.231505 E-01$ $0.186125 E-01$ MEV $=0.025$

$0.877859 E \quad 00$ $0.351734 E-00$ $0.185083 E-00$ $0.109508 E-00$ $0.694556 E-01$

$0.461852 E=01$ $0.318110 E-01$ $0.225200 \mathrm{E}-01$ $0.163049 E-01$ $0.120285 E-01$

\section{$0.901978 E-02$}

$0.686417 E-02$ $0.529781 E=02$

$0.732230 E 00$ $0.265001 E=00$ $0.129313 E=00$ $0.718858 \mathrm{E}-01$ $0.431920 E-01$

$0.273657 E-01$ $0.180372 \mathrm{E}-01$ $0.122619 E-01$ $0.854884 \mathrm{E}-02$ $0.608800 \bar{E}-02$

$0.441603 E-02$ $0.325857 E-02$ $0.244347 E-02$

\section{Polystyrene}

$0.397290 E-00$ $0.890826 E-01$ $0.303328 E-01$
$0.201060 E$ OI $0.823196 E$ O $0.443707 \mathrm{E}-00$ $0.261138 E=00$ $0.166713 E-00$

$0.110812 E=00$ $0.768367 \mathrm{E}-0$ $0.545785 \bar{E}-0$ $0.398179 E-01$ $0.295273 E-01$

$0.223107 E-01$ $0.170599 E=0$ $0.132297 \mathrm{E}-01$

$0.664555 E 00$ $0.192682 \mathrm{E}-00$ $0.801637 \mathrm{E}-01$ $0.387507 E-01$ $0.209460 E-01$

$0.121017 E-01$ $0.741666 E-02$ $0.472610 E-02$ $0.312595 E-02$

$0.212182 E=02$

$0.147814 E-02$

$0.104903 E-02$ $0.759006 E-03$ $0.124828 E-01$ $0.586828 E-02$

$0.300419 E-02$ $0.955440 E-03$ $0.578406 E-03$ $0.362143 E-03$

$0.234113 E-03$ $0.155050 E-03$ $0.105162 E-03$

\section{Polystyrene} $0.803874 E$ OO $0.431432 E=00$ $0.253508 E-00$ $0.161166 E-00$

$0.106820 E-00$ $0.737640 E-01$ $0.522256 E-0$ $0.379467 \mathrm{E}=01$ $0.280437 E-01$

$0.211050 E-01$ $0.160819 E-01$

$0.676108 E 00$ $0.198790 \mathrm{E}-00$ $0.830220 \mathrm{E}-0$ $0.402555 \mathrm{E}-0$ $0.217559 E-01$

$0.125691 E-01$ $0.769029 E-02$ $0.489374 E-02$ $0.322913 E-02$ $0.218735 E-02$

$0.151957 E-02$ $0.107580 E-02$ $0.124222 E=01$

$0.776040 E-03$

$0.412248 E-00$ $0.946863 E-01$ $0.326475 E-01$ $0.135597 E-01$ $0.640719 E=02$

$0.329339 E-02$ $0.181453 E-02$ $0.105106 E-02$ $0.636430 E-03$ $0.398529 E-03$

$0.257431 E-03$ $0.170368 E-03$ $0.115385 E-03$

\section{Polystyrene}

- 0 0.198551E 01 $0.828097 E$ EO $0.446433 E=00$ $0.263871 E=00$ $0.168325 E=00$

$0.112019 \mathrm{E}-00$ $0.775700 E=01$ $0.550934 E-01$ $0.401240 E=01$ $0.297287 E-01$

$0.224174 E-01$ $0.171185 E=0$

$0.132454 E-01$

\section{$0.669579 E \quad 00$ $0.197590 E=00$ $0.824925 E-01$ $0.400675 E-01$ $0.216669 E=01$ \\ $0.125381 E-01$ $0.767892 E-02$ $0.489421 E-02$ $0.323303 E-02$ $0.219319 E-02$ \\ $0.152530 E-02$ $0.108130 E-02$} MEV $=0.20$

0

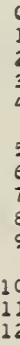

$0.196823 E \quad 01$ $0.827434 E$ OD $0.445861 E=00$ $0.263951 E=00$ $0.168248 E-00$

$0.111992 E-00$ $0.774780 E-01$ $0.550076 E-01$ $0.400185 E=0$ $0.296299 E=01$

$0.223166 \varepsilon-01$ $0.170262 E-01$ $0.131575 E=01$
$0.403755 E-00$ $0.923083 E-01$ $0.316549 E-01$ $0.131122 E-01$ $0.617353 E-02$

$0.317168 E-02$ $0.174508 E-02$ $0.101032 E-02$ $0.611391 E-03$ $0.382325 E-03$

$0.247 .238 E-03$ $0.163648 E-03$

Polystyrene

$0.673091 E 00$ $0.200379 E-00$ $0.838140 E=0$ $0.408071 E-01$ $0.220726 E-0$

$0.127821 E=01$ $0.782573 E-02$ $0.498778 E-02$ $0.329266 E-02$ $0.223273 E-02$

$0.155145 E-02$ $0.109908 E-02$ $0.792850 E-03$

$0.408310 E=00$ $0.943671 \mathrm{E}-01$ $0.325044 E-01$ $0.135166 E-01$ $0.637996 E-02$

$0.328074 E-02$ $0.180621 E-02$ $0.104647 E-02$ $0.633287 E-03$ $0.396593 E-03$

$0.256043 E-03$ $0.169437 E-03$ $0.114689 E-03$ $0.165203 E-02$ $0.110836 E-03$

$0.639936 E 00$ $0.213940 E-00$ $0.982693 E-01$ $0.519597 E-01$ $0.298836 E-01$

$0.182047 E-01$ $0.115759 E=01$ $0.761225 E-02$ $0.514511 E-02$ $0.355904 E-02$

$0.251239 E-02$ $0.180681 E-02$ $0.132303 E-02$

$0.283208 E-00$ $0.517516 E-01$ $0.149483 E-01$ $0.536575 E-02$

$0.223599 E-02$

$0.102902 E-02$ $0.513737 E-03$ C. $272199 E-03$ $0.152009 E-03$ $0.883601 E=04$

$0.533032 E-04$ $0.331001 E-04$ $0.211319 E-04$

$0.297120 E-00$ $0.560715 E-01$ $0.165233 E-01$ $0.602114 E-02$ $0.253499 E-02$

$0.117636 E-02$ $0.590653 \bar{E}=03$ $0.314452 \mathrm{E}-03$ $0.176154 E-03$ $0.102669 E-03$

$0.620279 E-04$ $0.385676 E-04$ $0.246329 E-04$

$0.289198 E-00$ $0.539872 \mathrm{E}-0$ ! $0.157539 E-01$ $0.570307 E=02$ $0.238678 E-02$

$0.110295 E-02$ $0.551700 \mathrm{E}-\mathrm{O}_{3}$ $0.292931 E-03$ $0.163693 E-03$ $0.952399 E-04$

$0.574443 E-04$ $0.356760 E-04$ $0.227597 E-04$

$0.293439 E-00$

$0.573129 E \quad 00$ $0.179097 \mathrm{E}-00$ $0.781190 E-01$ $0.395578 E-01$ $0.219055 E-01$

$0.128973 E-01$ $0.794876 E-02$ $0.507780 E-02$ 
Toble 2. Spotial Moments, Plone Perpendicular Source-Continued

n $\quad p=-1 / 2$

$1 / 2$

$3 / 2$

$5 / 2$

$7 / 2$

Polystyrene

$\ell=0 \begin{array}{r}0 \\ 1 \\ 2 \\ 3 \\ 4 \\ \\ 5 \\ 6 \\ 7 \\ 8 \\ 9 \\ 10 \\ 11 \\ 12\end{array}$

MEV $=0.40$

$0.679408 E-00$
$0.205567 E-00$
$0.866848 E-01$
$0.425353 E-01$
$0.231196 E-01$
$0.134529 E-02$
$0.626359 E-02$
$0.528440 E-02$
$0.349683 E-02$
$0.237700 E-02$
$0.165469 E-02$
$0.117443 E-02$
$0.848400 E-03$

$0.416582 E-00$
$0.982038 E-01$
$0.342392 E-01$
$0.143888 E-01$
$0.684214 E-02$
$0.354210 E-02$
$0.195993 E-02$
$0.114089 E-02$
$0.692953 E-03$
$0.435476 E-03$
$0.281925 E-03$
$0.187067 E-03$
$0.126895 E-03$

$0.301288 E-00$ $0.582699 \mathrm{E}-01$ $0.173861 \mathrm{E}-01$ $0.641235 \mathrm{E}-02$ $0.271894 E-02$

$0.127108 E-02$ $0.641266 \mathrm{E}-03$

$0.343167 \mathrm{E}-0$ $0.192904 E-03$

$0.112859 E-03$

$0.683580 E-04$ $0.426238 E-04$ $0.272738 \mathrm{E}-04$

Polystyrene

0. $188873 E$ OI $0.821698 \mathrm{E} \mathrm{OO}$ $0.449039 E-00$ $0.270091 E-00$ $0.173874 \mathrm{E}=00$

$0.116949 E-00$ $0.315179 \mathrm{E}-01$ $0.583284 \mathrm{E}-01$ $0.426917 \mathrm{E}-01$ $0.318064 E-01$

$0.240770 E=01$ $0.184644 E-01$ $0.143304 E-01$

\begin{abstract}
$0.689809 E 00$ $0.213622 E-00$ $0.914614 \mathrm{E}-01$ $0.454823 E-01$ $0.249697 E-01$

$0.146605 E-01$ $0.907091 E-02$ $0.583969 E-02$ $0.388627 E-02$ $0.265591 \mathrm{E}-02$

$0.185751 E-02$ $0.132428 E-02$
$0.960467 E-03$
\end{abstract}

0.430416 E-00 $0.104538 \mathrm{E}-00$ $0.372402 E-01$ $0.159361 E-01$ 0.768627 E-02 $0.225193 E-02$ $0.132290 E-02$ $0.809806 E-03$

$0.333934 E-03$ $0.222882 \mathrm{E}-03$

$0.151986 E-03$
$0.402856 E-02$
$M E V=1.00$

$0.184048 E$ OI $0.814119 E$ OO $0.449603 E=00$ $0.177121 \mathrm{E}-00$

0.119976 E-00 $0.841304 E-01$ $0.605445 E-01$ $0.445362 E-01$ $0.333450 E=02$

$0.253519 E-01$ $0.195277 \mathrm{E}-0$ $0.152147 E-01$ $0.273043 E-00$

Polystyrene

$\begin{array}{ll}0.700436 E-00 & 0.444834 E-00 \\ 0.221640 E-00 & 0.111186 E-00 \\ 0.964364 E-01 & 0.405036 E-01 \\ 0.486097 E-01 & 0.176562 E-01 \\ 0.269796 E-01 & 0.864620 E-02 \\ 0.159932 E-02 & 0.459163 E-02 \\ 0.997559 E-02 & 0.259537 E-02 \\ 0.646998 E-02 & 0.154000 E-02 \\ 0.433343 E-02 & 0.950908 E-03 \\ 0.297970 E-02 & 0.606766 E-03 \\ 0.209518 E-02 & 0.398109 E-03 \\ 0.150161 E-02 & 0.267522 E-03 \\ 0.109416 E-02 & 0.183525 E-03\end{array}$

Polystyrene

$M E V=2.00$ $0.441878 E-00$ $0.275416 E=00$ $0.182396 E-00$

$0.125893 E-00$ $0.897232 E-01$ $0.655413 E=0$ $0.488675 E-0$ $0.370507 \mathrm{E}-01$

$0.736399 E \quad 00$
$0.248071 E-00$
$0.113219 E-00$
$0.594207 E-01$
$0.340732 E-01$
$0.207742 E-01$
$0.132727 E-01$
$0.879372 E-02$
$0.600246 E-02$
$0.419872 E-02$
$0.299906 E-02$
$0.218062 E-02$
$0.161047 E-02$

$0.285008 E-0$ $0.221948 E-01$

$0.161047 E-02$

\section{$0.495786 E-00$ $0.135372 \mathrm{E}-00$ $0.528241 E-01$ $0.243831 E-01$ $0.125158 \mathrm{E}-01$}

$0.691972 \mathrm{E}-02$ $0.404876 E=02$ $0.247640 E-02$ $0.157077 \mathrm{E}=02$ $0.102674 E-02$

$0.688570 E-03$ $0.472010 E-03$ $0.329832 E-03$

\section{Polystyrene}

$0.151748 E \quad$ OI $0.731808 E$ OO $0.433623 E-00$ $0.280941 E-00$ $0.192399 E=00$

$0.136871 E-00$ $0.100227 E=00$ $0.750644 E-01$ $0.572692 \mathrm{E}-01$ $0.443653 E-01$

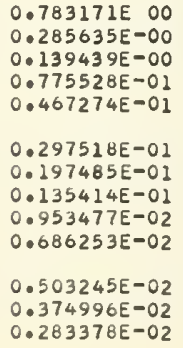

$0.567284 E \quad 00$ $0.173290 E-00$ $0.741694 E-01$ $0.370364 E-01$ $0.203334 E-01$

$0.119217 E-01$ $0.734549 E-02$ $0.470536 E-02$ $0.311150 E-02$ $0.211255 E-02$

$0.146695 \mathrm{E}-02$ $0.103852 E-02$ $0.747765 E-03$
$0.314279 E-00$ $0.630050 \mathrm{E}-01$ $0.193127 \mathrm{E}-01$ $0.728596 E-02$ $0.314638 \mathrm{E}-02$

$0.149438 E-02$ $0.764017 \mathrm{E}-03$ C. 413720 E-03 $0.234954 \mathrm{E}-03$ 0.138742 E-03

$0.847272 \mathrm{E}-04$ $0.532327 E-04$ $0.342937 \mathrm{E}-04$

$0.328097 E-00$ $0.681030 E-01$ $0.214627 \mathrm{E}=01$ $0.828626 \mathrm{E}-02$ $0.364806 \mathrm{E}-02$

$0.176189 \mathrm{E}-02$ $0.913742 E-03$ $0.501177 \mathrm{E}-03$ $0.287810 E=03$ $0.287810 E-03$
$0.171710 E-03$

$0.105814 E-03$ $0.670508 E-04$ $0.435264 E-04$

$0.378345 E-00$ $0.876350 E-01$ $0.301218 E-01$ $0.125041 \mathrm{E}-01$ $0.584913 \mathrm{E}-02$

$0.297612 \mathrm{E}-02$ $0.161471 E-02$ $0.921521 E-03$ $0.548213 E-03$ $0.337576 \mathrm{E}-03$

$0.214085 E-03$ $0.139242 \mathrm{E}-03$ 0.925913 E-04

$0.452523 E-00$ $0.120430 \mathrm{E}-00$ $0.463857 \mathrm{E}-01$ $0.212141 \mathrm{E}-01$ $0.107867 \mathrm{E}-01$

$0.590457 E-02$ $0.341742 E-02$ $0.206655 \mathrm{E}-02$ $0.129530 \mathrm{E}-02$ $0.836494 \mathrm{E}-03$

$0.554150 \mathrm{E}-03$ $0.375251 \mathrm{E}=03$ $0.259044 E-03$

$0.236082 E-00$
$0.387641 E-01$
$0.101288 E-01$
$0.333540 E-02$
$0.127865 E-02$
$0.545877 E-03$
$0.253454 E-03$
$0.125668 E-03$
$0.658159 E-04$
$0.360529 E-04$
$0.205322 E-04$
$0.120833 E-04$
$0.732143 E-05$

$0.194204 E-00$ $0.277105 \mathrm{E}-01$ $0.644774 E-02$ $0.192022 \mathrm{E}-02$ $0.672630 E-03$

$0.264548 E-03$ $0.113892 \mathrm{E}-03$ $0.526551 E-04$ $0.258352 E-04$ $0.133142 \mathrm{E}-\mathrm{C} 4$

$0.715949 \mathrm{E}-05$ $0.399151 E-05$

$0.229777 \mathrm{E}=05$

$0.247924 E-00$ $0.423819 E-01$ $0.114292 \mathrm{E}-01$ $0.386524 E-02$ $0.151478 E-02$

$0.659181 E-03$ $0.311100 E-03$ $0.156505 E-03$ $0.830115 E-04$ $0.459971 \mathrm{E}-04$

$0.264641 E-04$ $0.157209 E-04$ $0.960641 \mathrm{E}-05$

$0.260535 \mathrm{E}-00$ $0.463432 E-01$ $0.129077 \mathrm{E}-01$ $0.448550 E-02$ $0.179881 \mathrm{E}-02$

$0.798673 E-03$ $0.383527 E-03$ $0.195968 \mathrm{E}-03$ $0.105370 E-03$ $0.591225 E-04$

$0.343958 E=04$ $0.206467 \mathrm{E}-04$ $0.127346 \mathrm{E}=04$

$0.204863 \mathrm{E}-00$ $0.305505 \mathrm{E}-01$ $0.736498 \mathrm{E}-02$ $0.226061 \mathrm{E}-02$ $0.812234 \mathrm{E}=03$

$0.326625 E-03$ $0.143340 \mathrm{E}-03$ $0.674124 E=04$ OE -0

$0.954904 E-05$ $0.538375 E-05$ $0.313090 E-05$

$0.216291 \mathrm{E}-00$ $0.336989 E-01$ $0.842320 E=02$ $0.266615 E-02$ $0.983549 E-03$

$0.404798 \mathrm{E}-03$ $0.181273 E-03$ $0.868180 E-04$ $0.439439 E=04$ $0.232992 E-04$

$0.128508 \mathrm{E}-04$ $0.733497 \mathrm{E}-05$ $0.431312 E-05$

$0.307342 E-00$ $0.620705 E=01$ $0.191457 E-01$ $0.725072 \mathrm{E}-02$ $0.322760 E=02$

$0.147918 E-02$ $0.750589 E-03$ $0.402687 E-03$ $0.226163 E=03$ $0.131965 \mathrm{E}-03$

$0.795564 E-04$ $0.493279 E=04$ $0.313482 E=04$

$0.379038 E-00$ $0.898954 E-01$ $0.316462 E-01$ $0.134169 E-01$ $0.638228 \mathrm{E}-02$

$0.329014 E-02$ $0.180244 E-02$ $0.103592 E-02$ $0.619219 E-03$ $0.382465 E-03$

$0.242940 E=03$ $0.158086 E-03$ 
Table 2. Spatial Moments, Plane Perpendicular Source-Continued

$n$

$$
p=-1 / 2
$$

MEV $=10.00$

$$
1 / 2
$$

$$
3 / 2
$$$$
\text { Polystyrene }
$$

$0.851004 E=0$
$0.345512 E-00$
$0.186092 E-00$
$0.113084 E=00$
$0.737334 E-01$
$0.504078 E-01$
$0.356836 E-01$
$0.259515 E-01$
$0.192895 E=01$
$0.145985 E-01$
$0.112186 E-01$
$0.873530 E-02$
$0.688028 E-02$

\begin{abstract}
$0.682461 E 00$ $0.243552 \mathrm{E}-00$ 0.119109 E-00 $0.668626 E-01$ 0.407113 E-01

$0.261891 E-01$ $0.175444 \mathrm{E}-01$ $0.121292 \mathrm{E}=01$ $0.860163 E-02$ $0.623010 E-02$

$0.459397 E-02$

$0.344016 E-02$ $0.261113 E-02$
\end{abstract}

$0.580966 E 00$ $0.187640 E-00$
$0.850245 E-01$ $0.447947 E-01$

$0.258085 E-01$

$0.158013 E-01$

$0.101189 E-01$

$0.671038 E-02$

$0.457,767 E-02$

$0.319697 \mathrm{E}-02$

\section{$0.227768 E-02$}

$0.165086 E-02$
$0.121470 E-02$
$0.510111 E 00$

$0.151512 E-00$ $0.643354 E-01$ $0.320986 E=01$

$0.1763325-01$

$0.103435 E-01$ $0.636947 E-02$ $0.407355 E-02$ $0.268631 E-02$ $0.181719 E-02$

$0.125617 E-02$ $0.884721 E-03$ $0.633399 E-03$
$0.456785 E-00$

$0.126091 E-00$

$0.505532 \mathrm{E}-01$

$0.240306 E-01$

$0.126517 E-01$

$0.714256 E-02$

$0.424665 \mathrm{E}-02$

$0.262891 E-02$

$0.168159 \mathrm{E}-02$

$0.110531 E-02$

$0.743534 E-03$

$0.510269 E-03$

$0.356380 E-03$
$0.414738 E-00$ $0.107215 E-00$ $0.408114 E-01$ 0.185656 E- U1 $0.940323 E-02$

$0.512630 E-02$ $0.295163 E-02$ $0.177355 E-02$ $0.110319 E-02$ $0.706255 E-03$

$0.463352 \mathrm{E}-03$ $0.310494 E-03$ $0.211966 E-03$ 


\section{References}

[1] L. V. Spencer, Phys. Rev. 98, 1597 (1955).

[2] Ann T. Nelms, NBS Circ. 577 (1956).

[3] Ann T. Nelms, Supplement to NBS Circ. 577 (July 30, 1958).

[4] R. M. Sternheimer, Phys. Rev. 88, 851 (1952); 91, 256 (1953).

[5] R. Mather and Segré, Phys. Rev. 84, 191 (1951).

[6] J. A. Doggett and L. V. Spencer, Phys. Rev. 103, 1597 (1956).

[7] G. Moliere, Z. Naturforsch. 2A, 133 (1947).

[8] U. Fano, Phys. Rev. 93, 117 (1954).

[9] F. Frantz (private communication). See [1].

[10] Clark, Brar, and Marinelli, Radiology 64, 94 (1955).

[11] R. Loevinger, Radiology 66, 55 (1955).

[12] F. N. Huffman: ORNL Report 2137, available from Office of Technical Services, U.S. Department of Commerce, Washington, D.C. See also Huffman, Cheka, Saunders, Ritchie, and Birkhoff, Phys. Rev. 106, 435 (1957).

[13] I. D. Birkhoff, The Passage of Fast Electrons through Matter, Encyclopedia of Physics vol. 34, Springer-Verlag, 1958.

[14] A. E. Grün, Z. Naturforsch, 12a, Heft 2, 89 (1957).

[15] J. G. Trump, K. A. Wright, and A. M. Clark, J. Appl. Phys. 21, 345 (1950).

Washington, February 10, 1959. 

